\title{
Tyrosine kinase inhibitors for cancer treatment
}

Citation for published version (APA):

Tullemans, B. M. E. (2021). Tyrosine kinase inhibitors for cancer treatment: effects on platelets. [Doctoral Thesis, Maastricht University]. ProefschriftMaken. https://doi.org/10.26481/dis.20211006bt

Document status and date:

Published: 01/01/2021

DOI:

10.26481/dis.20211006bt

Document Version:

Publisher's PDF, also known as Version of record

\section{Please check the document version of this publication:}

- A submitted manuscript is the version of the article upon submission and before peer-review. There can be important differences between the submitted version and the official published version of record.

People interested in the research are advised to contact the author for the final version of the publication, or visit the DOI to the publisher's website.

- The final author version and the galley proof are versions of the publication after peer review.

- The final published version features the final layout of the paper including the volume, issue and page numbers.

Link to publication

\footnotetext{
General rights rights.

- You may freely distribute the URL identifying the publication in the public portal. please follow below link for the End User Agreement:

www.umlib.nl/taverne-license

Take down policy

If you believe that this document breaches copyright please contact us at:

repository@maastrichtuniversity.nl

providing details and we will investigate your claim.
}

Copyright and moral rights for the publications made accessible in the public portal are retained by the authors and/or other copyright owners and it is a condition of accessing publications that users recognise and abide by the legal requirements associated with these

- Users may download and print one copy of any publication from the public portal for the purpose of private study or research.

- You may not further distribute the material or use it for any profit-making activity or commercial gain

If the publication is distributed under the terms of Article $25 \mathrm{fa}$ of the Dutch Copyright Act, indicated by the "Taverne" license above, 


\title{
Tyrosine kinase inhibitors for cancer treatment: Effects on platelets
}

\author{
Bibian M.E. Tullemans
}


Tyrosine kinase inhibitors for cancer treatment: Effects on platelets

Thesis: Maastricht University

ISBN: 978-94-6423-418-3

Production: ProefschrifMaken || www.proefschriftmaken.nl

(C) Bibian Tullemans, Maastricht 2021

Cover design by Bregje Jaspers of STUDIO 0404 || www.proefschriftontwerp.nl 


\title{
Tyrosine kinase inhibitors for cancer treatment: Effects on platelets
}

\author{
Proefschrift \\ Ter verkrijging van de graad van doctor aan de Universiteit Maastricht, \\ op gezag van de Rector Magnificus, Prof. Dr. Rianne M. Letschert, \\ volgens het besluit van het College van Decanen, \\ in het openbaar te verdedigen op
}

Woensdag 06 oktober 2021 om 16.00 uur

door

Beatrice Maria Emanuelle Tullemans

Geboren op 27 oktober 1992 te Weert 


\section{Promotor}

Prof. Dr. J.W.M. Heemskerk

\section{Copromotor}

Dr. M.J.E. Kuijpers

Dr. M.J.B. Aarts

\section{Beoordelingscommissie}

Prof. Dr. H. ten Cate (Voorzitter)

Prof. Dr. G.M.J. Bos

Dr. S. Séverin (Université Paul Sabatier, France)

Prof. Dr. J.C. Sluimer

Dr. A.A.M. van der Veldt (Erasmus Medisch Centrum)

The research described in this thesis was partially supported by a research grant from Pfizer (WI209458)

Financial support for the publication of this thesis by Pfizer is gratefully acknowledged.

Financial support for the publication of this thesis by Hart Onderzoek Nederland is gratefully acknowledged. 


\section{Contents}

Chapter 1 General Introduction

Chapter 2 Acquired platelet antagonism: off-target antiplatelet effects of malignancy treatment

Chapter 3 Tyrosine kinase inhibitor pazopanib inhibits platelet procoagulant activity in renal cell carcinoma patients

Chapter 4 Tyrosine kinase inhbitor sunitinib delays platelet-induced coagulation: additive effects of aspirin

Chapter 5 Quantitative and qualitative changes in platelet traits of sunitinibtreated patients with renal cell carcinoma in relation to circulating sunitinib levels: a proof-of-concept study

Chapter 6 Comparison of inhibitory effects of irreversible and reversible Btk inhibitors on platelet function

Chapter 7 Multiparameter screening for platelet-inhibitory effects of tyrosine kinase inhibitors used for cancer treatment

Chapter 8 General Discussion

Chapter 9 Summary

Samenvatting

Impact

Curriculum Vitae

Publications

Acknowledgements 

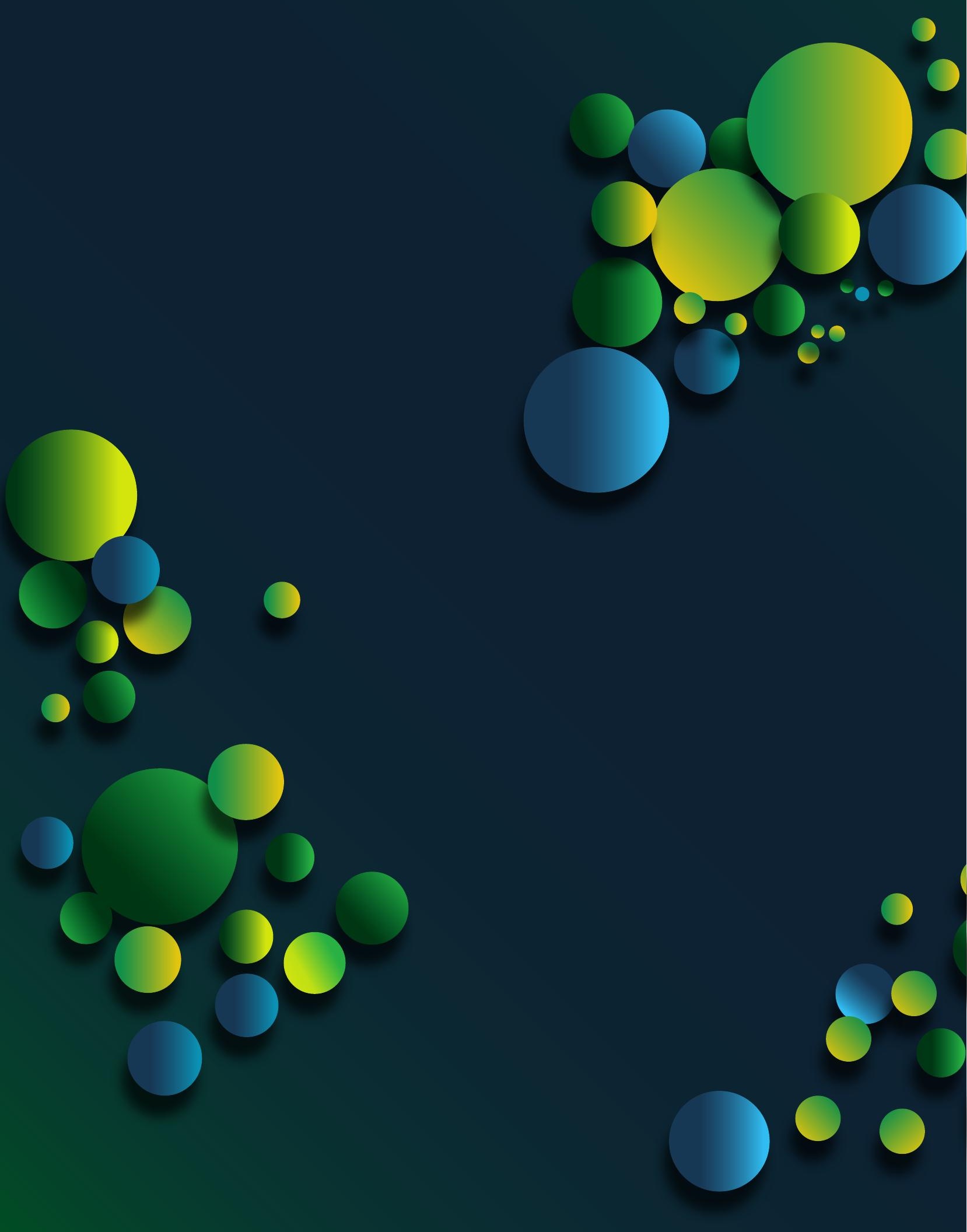


\section{Chapter 1}

General Introduction

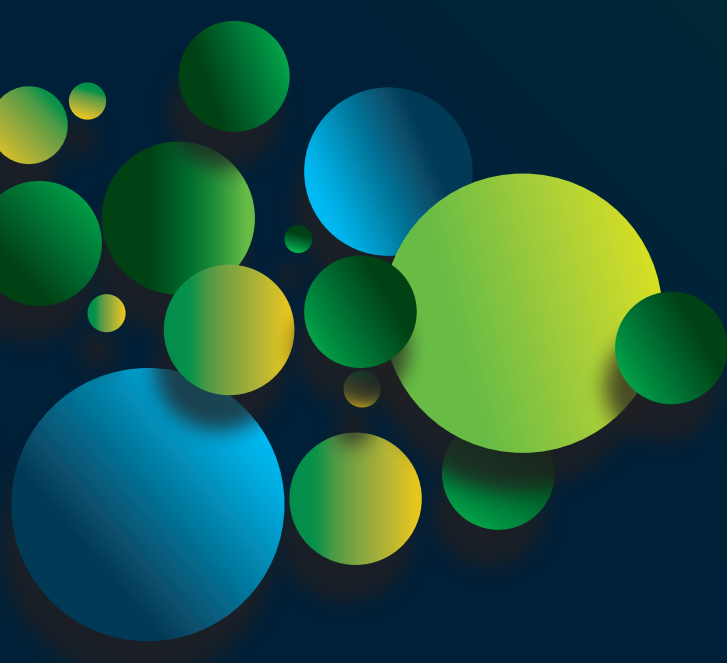


Platelets are known to play a crucial role in haemostasis ${ }^{1-3}$. Upon damage to the vessel wall, platelets become activated via different receptors and adhere to the site of injury. Subsequently, a platelet plug is formed, which is stabilised by the formation of fibrin, preventing further blood loss. Unbalanced haemostasis by impaired platelet function or increased platelet reactivity contributes to pathological conditions that involve bleeding or thrombosis, respectively. Yet, platelets are also described to be involved in tumour angiogenesis and cancer progression ${ }^{4,5}$. In this chapter, a general background is provided of the different receptors that mediate platelet activation and the involvement of tyrosine kinases in this process. Furthermore, a brief overview is given on the role of platelets in malignant disease and the development of tyrosine kinase inhibitors as targeted therapeutic drugs.

\section{Involvement of tyrosine kinases in platelet signalling}

For decades, the involvement of platelet function in haemostasis has been thoroughly investigated, which resulted in the knowledge we have today ${ }^{1-3}$. The process of haemostasis is initiated by vessel wall disruption and the exposure of thrombogenic components in the underlying matrix. Platelets can interact with these immobilised and other soluble ligands with a variety of receptors that induce shape change and adhesion to the vessel wall. Ensuing platelet activation can be triggered by different receptors interacting with ligands exposed upon injury. In the following sub-sections, a short description is provided of the major signalling pathways of platelet activation and the tyrosine kinases involved in these processes.

\section{GPIb-IX-V signalling}

Tethering of platelets along the vessel wall is initiated by collagen-bound von Willebrand Factor (VWF) through the glycoprotein (GP)Ib-IX-V complex (Figure 1.1). This shear-dependent interaction causes platelet rolling, which enables subsequent firm adhesion to the site of injury via collagen $^{6}$. The interaction between VWF and the GPIba subunit in the GPIb-IX-V complex leads to weak downstream signalling events. The adapter protein 14-3-3 3 supports downstream signalling and the phosphorylation of Src family kinases (SFK) SrC and Lyn ${ }^{7,8}$. In turn, phosphatidylinositol 3-kinase (PI3K) is activated leading to activation of integrins, stabilised VWF binding, granule secretion and adhesion to the surface. In addition, GPIb-IX-V signalling can also be associated with Fc receptor $\gamma$-chain (FcRY) or FcRplla induced activation through the immunoreceptor tyrosinebased activation motif (ITAM) in this receptor ${ }^{8}$. Phosphorylation of the FcRY (or FcRylla) leads to the binding of Syk and induces a cascade of signals comparable to the GPVI receptor (described in the following paragraph). In general, GPIb-IX-V initiates only a limited activation signal in platelets, resulting in transient $\mathrm{Ca}^{2+}$ oscillations ${ }^{6}$, which mainly helps to induce and amplify platelet activation through other receptors ${ }^{7,9}$. 


\section{GPVI signalling}

As platelets roll along the damaged vessel wall, GPVI is able to interact with collagen (Figure 1.1). Although GPVI is most efficiently activated by collagen (type I or III), also other ligands have been described to activate this receptor, including physiological (laminin, fibronectin, vitronectin and fibrin(ogen)), synthetic ligands (collagen-related peptide, CRP) or the snake venom toxin convulxin ${ }^{10}$. GPVI is a member of the immunoglobulin superfamily and is associated with the dimeric immunoreceptor tyrosine-based activation motif (ITAM)-containing FcRy receptor, 11, 12. Activation of GPVI leads to the phosphorylation of the SFKs Fyn and Lyn, which in turn induces clustering of GPVI and phosphorylation of the ITAM motif ${ }^{1,8}$. This leads to the binding and activation of Syk that will initiate the formation of a complex involving linker for activated T-cells (LAT), Tec family kinases (Btk, Tec), PI3K, phospholipase C- $\gamma 2$ (PLCY2) and several other proteins $^{1,2,13}$. Through a cascade of signals, calcium increases in the cytosol, promoting processes of platelet activation. As stimulation of GPVI induces strong platelet activation with high sustained calcium elevation, it can promote integrin activation, granule secretion, thromboxane production and procoagulant activity (exposure of phosphatidylserine (PS)), resulting in platelet adhesion, aggregation, and thrombus formation ${ }^{2,14}$.

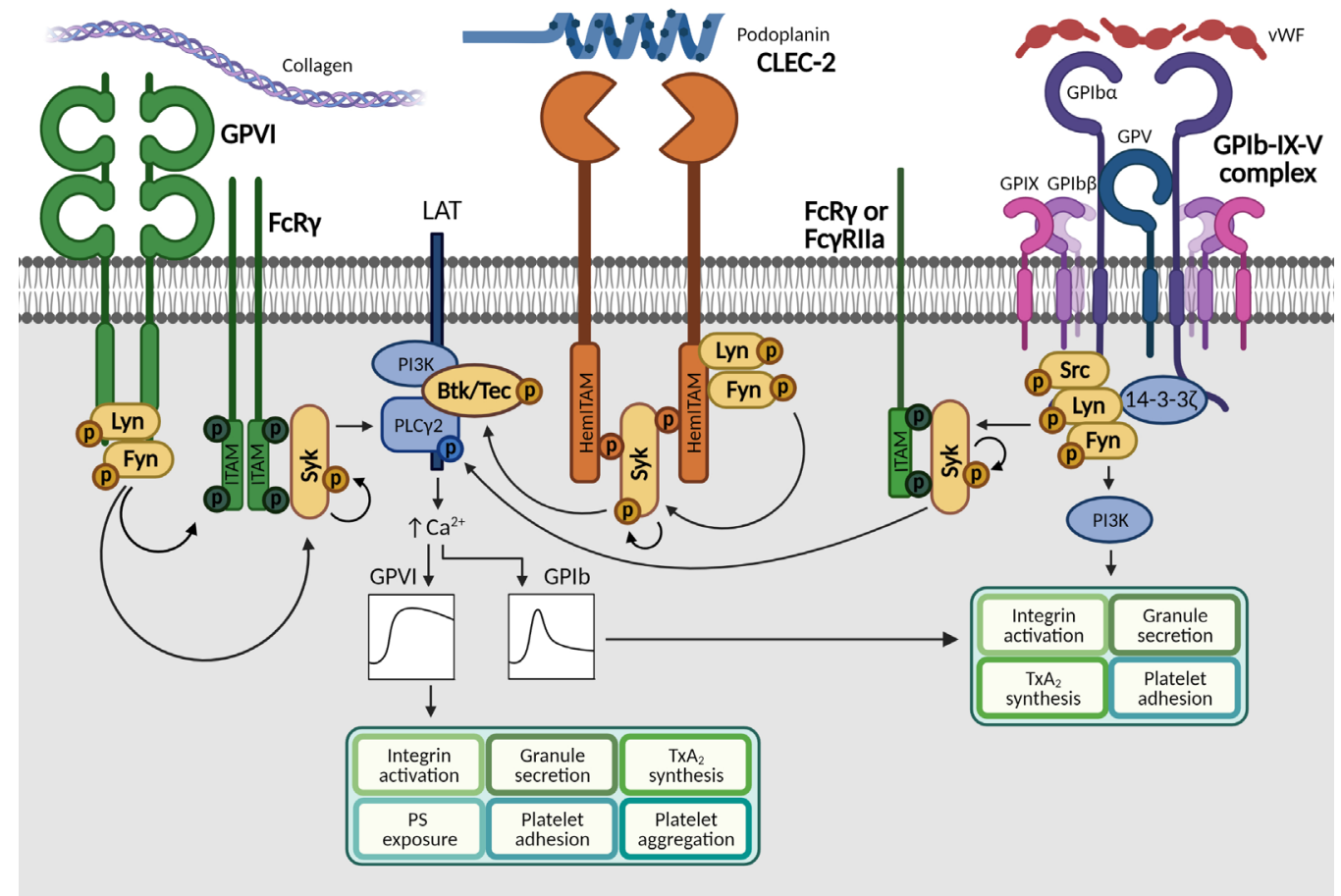

Figure 1.1. Schematic overview of signalling cascades upon activation via collagen receptor GPVI, podoplanin receptor CLEC-2 and von Willebrand factor (vWF) receptor GPIb-IX-V. Shown are the most potent agonists for each receptor. For abbreviations: see text. Modified after references ${ }^{1,2,7,8,12,13}$. 


\section{CLEC-2 signalling}

A less well studied receptor that mediates platelet activation is C-type lectin-like type II membrane glycoprotein (CLEC-2) (Figure 1.1). CLEC-2 is activated through its natural ligand podoplanin or the snake venom toxin rhodocytin ${ }^{15,16}$. The CLEC-2 receptor contains a single hemi-immunoreceptor tyrosine-based activation motif (hemITAM), and its signalling involves many molecules that are also present in the GPVI pathway. Upon activation the hemITAM motif becomes phosphorylated through Fyn and Lyn (and possibly Btk or Tec)1, 13, 17. This enables dimerization of CLEC-2 and the binding of Syk to the hemITAM motif, leading to the downstream signalling through the LAT complex, inducing an increase of intracellular calcium and resulting in platelet activation ${ }^{1,2}$. Although GPVI and CLEC-2 signalling are in many ways alike, CLEC-2 signalling appears to be more reliant on Syk, whereas GPVI signalling is more SFK dependent ${ }^{18}$.

\section{Integrin activation}

Upon platelet activation, one of the induced responses is the activation of integrins through inside-out signalling (Figure 1.2A) ${ }^{1}$ 19. Integrins are transmembrane proteins consisting of an $\alpha$ and $\beta$ chain that exist as a noncovalent heterodimeric complex. Integrins are important for stable adhesion to the vessel wall and platelet aggregate formation ${ }^{1}$. For integrins to become active, a conformational change in this complex is necessary to obtain a high-affinity ligand binding state ${ }^{2}$ ${ }^{20}$. In the low-affinity state of integrins, Csk forms a complex with Src and maintains the $\beta$-chain in an inactive state. Activation induces dissociation of Csk from the complex which leads to the activation of $\mathrm{Src}^{8,21}$. Subsequently, the actin cytoskeletal proteins talin-1 and kindlin-3 bind to $\mathrm{Src}$ and provoke the conformational change that leads to the increase in affinity (Figure 1.2A).

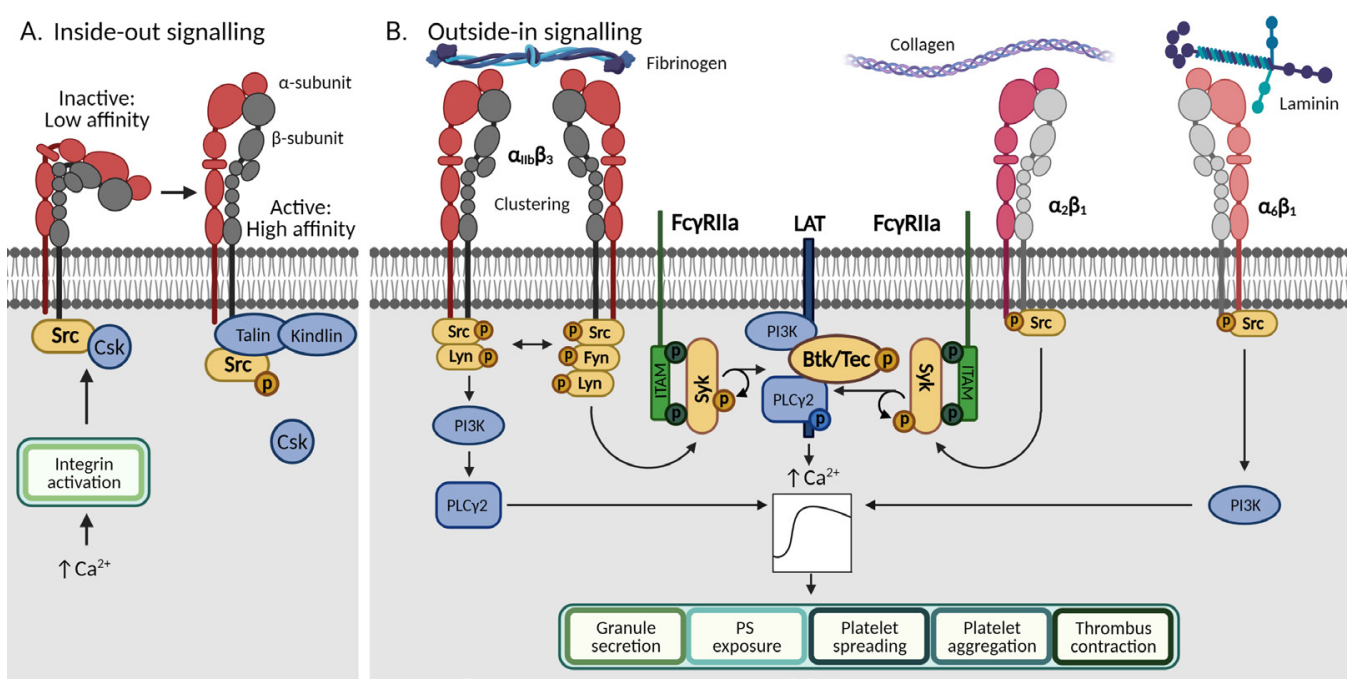

Figure 1.2. Schematic overview of (A) inside-out and (B) outside-in signalling of platelet integrins including $\alpha_{11 \mathrm{~b}} \beta_{3}$, $\boldsymbol{\alpha}_{2} \boldsymbol{\beta}_{1}$ and $\boldsymbol{\alpha}_{6} \boldsymbol{\beta}_{1}$. Shown are the most potent agonists for each receptor. For abbreviations: see text. Modified after references ${ }^{1,}$ 2, 8, 23, 31. 
This allows integrins to interact with several adhesive or soluble compounds ${ }^{1,2}$. There are several integrins expressed on platelets $\left(\alpha_{2} \beta_{1}, \alpha_{5} \beta_{1}, \alpha_{6} \beta_{1}, \alpha \vee \beta_{3}\right.$ and $\left.\alpha_{1 \mid b} \beta_{3}\right)$, but for this thesis background only the integrins $\alpha_{2} \beta_{1}, \alpha_{6} \beta_{1}$ and $\alpha_{11 b} \beta_{3}$ are relevant.

Integrin $\alpha_{\| \mathrm{lb}} \beta_{3}$ (GPIIb/IIla) is the most abundant (integrin) receptor on platelets with over 80,000 copies on the membrane and additional copies in the $\alpha$-granules ${ }^{22,23}$. Several ligands can interact with activated integrin $\alpha_{\| 1} \beta_{3}$, including fibrin, fibrinogen, VWF and fibronectin ${ }^{21,23}$. Ligand binding to integrin $\alpha_{\| b} \beta_{3}$ contributes to platelet activation via a process called outside-in signalling that relies on clustering of the receptor (Figure 1.2B) ${ }^{1,2}$. This activates a cascade of events that involves the activation of the SFKs Src, Lyn and/or Fyn. This can result in direct stimulation of PI3K and PLCY2 signalling, although this can also occur indirectly via the activation of the ITAM signalling pathway involving Syk ${ }^{2,8,24}$. Both pathways result in an increase of intracellular calcium levels and drive processes such as platelet spreading, stable adhesion, aggregation, thrombus contraction and procoagulant activity of platelets ${ }^{23,25}$.

In comparison to integrin $\alpha_{1 \mid 16} \beta_{3}$, integrin $\alpha_{2} \beta_{1}$ (GPla/Ila) is less abundantly expressed with only 2,000-4,000 copies per platelet ${ }^{23}$. The ligands that integrin $\alpha_{2} \beta_{1}$ can interact with are fibrillar and soluble collagens (Figure 1.2B) ${ }^{1}$. The activation of outside-in signalling of $\alpha_{2} \beta_{1}$ is thought to be similar to that of $\alpha_{\| 1 b} \beta_{3}$ in which activation of Src leads to the activation of the Syk-dependent ITAM signalling ${ }^{23,26}$. The role of this integrin is believed to be mainly complimentary to GPVI via maintaining and stabilising platelet adhesion ${ }^{27-29}$.

The integrin $\alpha_{6} \beta_{1}$ is a moderately expressed protein with around 10,000 copies per platelet ${ }^{22}$. Integrin $\alpha_{6} \beta_{1}$ serves as a receptor for laminin (Figure 1.2B) and is described to be moderately important in thrombus formation ${ }^{30}$. It is suggested that activation of $\alpha_{6} \beta_{1}$ mainly involves the activation of Src and subsequently PI3K, resulting in cytoskeletal rearrangement and platelet spreading ${ }^{31}$.

\section{G-Protein Coupled Receptor signalling}

Another platelet activation response is the release of soluble autocrine agonists (e.g., ADP and thromboxane $\left.\mathrm{A}_{2}\left(\mathrm{TxA}_{2}\right)\right)$ at the site of injury. Several so-called secondary mediators, such as ADP, are secreted from the $\alpha$ - and $\delta$-granules upon activation. TxA $A_{2}$ is formed from arachidonic acid in the platelet membrane via several steps involving cyclooxygenase-1. In addition, thrombin is an important soluble platelet agonist that arises during coagulation. In general, these soluble agonists activate platelets via G protein-coupled receptors (GPCRs). GPCRs are transmembrane proteins with seven domains passing through the cell membrane ${ }^{32}$. Members of this family include the ADP receptors $P 2 Y_{1}$ and $P 2 Y_{12}$, the TxA 2 receptor (TP) and the receptors for thrombin, protease-activated receptor (PAR) 1 and $4^{27}$. Tyrosine kinases are not considered to be involved in the downstream signalling of these receptors, with the exception of the $\mathrm{P}_{2} \mathrm{Y}_{12}$ receptor (Figure 1.3). The $P 2 Y_{1}$, TP and PAR receptors all rely on downstream signalling via the G-proteins $G 12 / 13 \alpha$ 
and/or Gq $\alpha$. Activation via G12/13 $\alpha$ leads to activation of Rho-associated protein kinase (ROCK) resulting in cytoskeletal rearrangement and shape change in platelets ${ }^{33}$. On the other hand, Gq $\alpha$-induced signalling activates phospholipase $C \beta(P L C \beta)$ followed by intracellular calcium rises resulting in strong platelet responses such as integrin activation and granule secretion ${ }^{33}$. Only P2 $\mathrm{Y}_{12}$ is aberrant in its signalling as compared to the other GPCRs, as it is related to the G-protein $\mathrm{Gia}^{34}$. Stimulation of $\mathrm{P}_{2} \mathrm{Y}_{12}$ induces a cascade involving the activation of tyrosine kinases (Fyn and Lyn) and subsequently PI3K and Akt. Eventually, this results in integrin activation and aggregation of platelets. In addition, stimulation of Gia reduces CAMP, which inhibits platelet activation ${ }^{35}$.

\section{TAM signalling}

Another soluble ligand is Gas6, a plasma protein that is increased in response to inflammation, survival, growth arrest and other biological processes ${ }^{27}$. Furthermore, Gas6 is present in the $\alpha$-granules of platelets and released during activation ${ }^{36}$. Gas 6 is the ligand for the tyrosine kinase receptors Tyro3, Axl and Mer (TAM), which are important in clearance of apoptotic cells and resolution of inflammation ${ }^{37,38}$. In addition, a role of TAM in platelet aggregation and clot formation is reported ${ }^{38,39}$. It is believed that the activation of TAM synergizes with P2Y $Y_{12}$ signalling, thereby persisting $\alpha_{11 b} \beta_{3}$ activation and platelet aggregation, a pathway that is dependent on the phosphorylation of Akt through $\mathrm{PI}^{3} \mathrm{~K}^{38}$. Although not much is known with regard to the involvement of tyrosine kinases downstream of TAM receptors in platelets, TAM receptors are tyrosine kinases itself and are described to signal via Src and/or Fyn resulting in downstream signalling of PI3K, PLCY and ERK in other cells such as fibroblasts ${ }^{40-43}$. This might point towards a possible involvement of (one of) these kinases in this pathway.

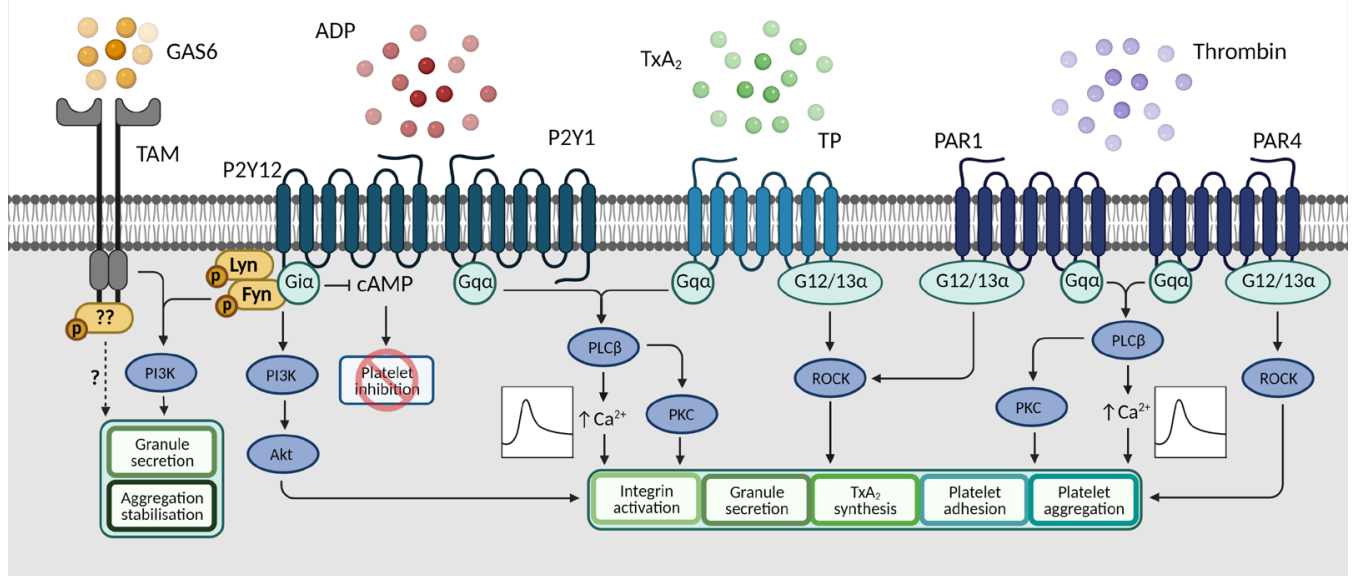

Figure 1.3. Schematic overview of signalling cascades upon platelet activation of the Gas6 receptors Tyro-3, AxI and Mer (TAM) and G-protein coupled receptors (GPCR), i.e. ADP receptors P2Y $Y_{12}$ and $P 2 Y_{1}$, thromboxane $A_{2}$ (TxA $A_{2}$ receptor TP and thrombin receptors protease-activated receptors (PAR)1 and 4. Shown are the most potent agonists for each receptor. For abbreviations: see text. Modified after references $2,8,33-35,38$. 


\section{Roles of platelets in malignant disease}

Platelets are not only important in haemostasis to prevent excessive bleeding or thrombosis, they are also involved in malignant diseases and inflammation ${ }^{44,45}$. It is known that cancer patients are at risk for thrombosis ${ }^{46,47}$, as aberrations in coagulation and haemostasis were observed in these patients more than 150 years ago ${ }^{48}$. Nowadays, the interactions between platelets and cancer have been more clearly described ${ }^{49}$. Solid tumours are known to induce thrombocytosis, an elevated platelet count $>400 \times 10^{9} / \mathrm{L}$, but tumour cells are also able to induce platelet activation ${ }^{4}$ 45 . Platelets (and their function) are suggested to be important in the growth of tumours and formation of metastasis via angiogenesis. Platelets contain many anti- and proangiogenic molecules in their granules which are secreted upon their activation. Platelets are also described as main transporters of vascular endothelial growth factor (VEGF), which is an important stimulus of angiogenesis ${ }^{50}$. Furthermore, platelets are able to sequester all kinds of plasma proteins into their granules through active endocytosis ${ }^{51}$. In order for platelets to release their granule content close to the tumour site, platelet activation is necessary. Tumour cells are known to express or secrete several proteins that are platelet-activating, such as thrombin, ADP and podoplanin ${ }^{52}$. Platelet activation through PAR1 and P2 $\mathrm{Y}_{12}$ is proposed to mainly induce the secretion of VEGF ${ }^{45}$. Other than VEGF, platelets are also able to release other pro-angiogenic growth factors, such as platelet-derived growth factor (PDGF) and transforming growth factor beta (TGF- $\beta$ ) from specific $\alpha$-granules. Release of these factors can promote angiogenesis, tumour neovascularization and growth $^{53,54}$. Besides stimulating angiogenesis and tumour growth, platelets can also be involved in tumour cell survival and metastasis ${ }^{52}$. Platelets express various adhesion molecules on their surfaces (integrins, selectins, etc.), allowing them to bind to tumour cells after entering into the blood vessels. This results in shielding and protection of the tumour cells from the immune system ${ }^{55,56}$, as well as the high shear forces in the blood stream ${ }^{57,58}$. In addition, platelets in these so-called platelet-tumour aggregates can also help in arrest and stabilisation of tumour cells to the endothelial wall of a distant blood vessel, hence further facilitating metastasis ${ }^{59}$. Altogether, this shows the involvement of platelets in tumour progression and metastasis.

\section{Off-target effects of tyrosine kinase inhibitors}

As more knowledge is gained on cancer progression and the underlying mechanisms, new types of therapeutic drugs have been developed to interfere with these processes, including angiogenesis. In the last decades, several types of tyrosine kinase inhibitors (TKIs) have been developed to target (multiple) receptor and/or downstream tyrosine kinases which are important in signalling pathways that mediate tumour progression. As these TKIs can be orally administered and are overall well tolerated in patients, increasing numbers are being developed and several have been approved for clinical use in several types of cancer ${ }^{27}$. However, given that TKIs inhibit important tyrosine kinases in cancer cells, due to a high tyrosine kinase expression in other cells 
such as platelets, "off-target" effects can be expected. Hence, for several TKIs (severe) side effects have been reported, while also cancers can become resistant toward these drugs.

\section{Aims and outlines of this thesis}

This thesis has as overall aim to provide better insight into the off-target effects of tyrosine kinase inhibitors (TKIs) used for cancer treatment on platelet functions. The general introduction in this Chapter provides background information on key pathways of platelet activation and the involvement of most relevant tyrosine kinases in these pathways. Chapter 2 reviews nearly 40 multi-target TKIs that are currently used for treatment of different types of cancer, to prolong progression-free survival. This chapter describes how several TKIs can inhibit activation mechanisms in platelets, and which are the clinical consequences of these antiplatelet effects. In Chapter 3, we investigated effects of the antiangiogenic compound pazopanib on platelet function in vitro and in blood of advanced renal cell carcinoma (RCC) patients during treatment. Patients treated with pazopanib are reported to have an increased mild bleeding risk. Given the well-established role of protein tyrosine kinases in platelet activation, we hypothesized that the increased bleeding risk could be due to an off-target effect by pazopanib on platelet tyrosine kinases. The focus of Chapter $\mathbf{4}$ is sunitinib, another multi-target TKI used for the treatment of advanced RCC. Sunitinib was already described to affect collagen-induced activation under noncoagulating conditions. We investigated the effects of sunitinib on thrombus formation induced by other tyrosine kinase-dependent receptors, as well as the effects under coagulating conditions in a whole blood microfluidics system. Since cancer patients often experience cardiovascular diseases as a co-morbidity, they are often on a combined therapy of sunitinib with antiplatelet or anticoagulant drugs. As both are associated with an increased bleeding risk, we examined the synergistic effects of sunitinib with aspirin, as a common antiplatelet drug, on thrombus and fibrin formation. In Chapter $\mathbf{5}$ we describe a patient study with sunitinib-treated RCC patients. Here we explored the effects of sunitinib-treatment on quantitative and qualitative platelet traits in relation to the sunitinib levels in plasma or serum, and also assessed the occurrence of bleeding to evaluate the haemostatic consequences and clinical relevance.

Several types of TKIs have also been reported to increase the bleeding risk, often lacking a clear explanation. It is of importance to investigate possible underlying antiplatelet mechanisms, to come to better-informed treatment decisions, especially when prescribed in combination with antiplatelet or anticoagulant drugs. Therefore, in Chapter 6, we investigated the effect of three Btk inhibitors (acalabrutinib, ibrutinib and MK-1026) on platelet function pathways in vitro and in patients. Furthermore, we assessed the off-target effects in Btk knock-out mice.

In the literature, the effects of other TKIs on platelets have only partially been assessed. The reported effects of several TKIs on the GPVI pathway in platelets make those TKIs, at a lower dose, interesting as possible antiplatelet drugs, since GPVI inhibition is reported not to be associated 
with an increased bleeding tendency. On the other hand, effects on other platelet signalling pathways remain largely unknown, which may possibly explain the reported increased risk of bleeding. To explore whether TKIs are of potential interest as antiplatelet drugs, we systematically investigated the effects of eight clinically used TKIs on (physiological) platelet function in whole blood under flow and isolated platelets using different agonists in Chapter 7. In the last chapter of this thesis, Chapter 8, the most important findings of this thesis are highlighted and brought in context with the current literature.

\section{References}

1. Versteeg HH, Heemskerk JW, Levi M, Reitsma PH. New fundamentals in hemostasis. Physiol Rev. 2013; 93: 327-58.

2. Estevez B, Du X. New concepts and mechanisms of platelet activation signaling. Physiology (Bethesda). 2017; 32: 162-77.

3. van der Meijden PEJ, Heemskerk JWM. Platelet biology and functions: new concepts and clinical perspectives. Nat Rev Cardiol. 2019; 16: 166-79.

4. Jain S, Harris J, Ware J. Platelets: linking hemostasis and cancer. Arterioscler Thromb Vasc Biol. 2010; 30: 2362-7.

5. Yeung J, Li W, Holinstat M. Platelet signaling and disease: targeted therapy for thrombosis and other related diseases. Pharmacol Rev. 2018; 70: 526-48.

6. Ruggeri ZM, Mendolicchio GL. Interaction of von Willebrand factor with platelets and the vessel wall. Hamostaseologie. 2015; 35: 211-24.

7. DuX. Signaling and regulation of the platelet glycoprotein Ib-IX-V complex. Curr Opin Hematol. 2007; 14: 262-9.

8. Senis YA, Mazharian A, Mori J. Src family kinases: at the forefront of platelet activation. Blood. 2014; 124: 2013-24.

9. Kasirer-Friede A, Cozzi MR, Mazzucato M, De Marco L, Ruggeri ZM, Shattil SJ. Signaling through GP Ib-IX-V activates alpha IIb beta 3 independently of other receptors. Blood. 2004; 103: 3403-11.

10. Harbi MH, Smith CW, Nicolson PLR, Watson SP, Thomas MR. Novel antiplatelet strategies targeting GPVI, CLEC-2 and tyrosine kinases. Platelets. 2021; 32: 29-41.

11. Nieswandt B, Watson SP. Platelet-collagen interaction: is GPVI the central receptor? Blood. 2003; 102: 449-61.

12. Damaskinaki FN, Moran LA, Garcia A, Kellam B, Watson SP. Overcoming challenges in developing small molecule inhibitors for GPVI and CLEC-2. Platelets. 2021: 1-9.

13. Rayes J, Watson SP, Nieswandt B. Functional significance of the platelet immune receptors GPVI and CLEC-2. J Clin Invest. 2019; 129: 12-23.

14. Munnix IC, Kuijpers MJE, Auger J, et al. Segregation of platelet aggregatory and procoagulant microdomains in thrombus formation: regulation by transient integrin activation. Arterioscler Thromb Vasc Biol. 2007; 27: 2484-90.

15. Navarro-Núñez L, Langan SA, Nash GB, Watson SP. The physiological and pathophysiological roles of platelet CLEC-2. Thromb Haemost. 2013; 109: 991-8.

16. Suzuki-Inoue K, Inoue O, Ozaki Y. Novel platelet activation receptor CLEC-2: from discovery to prospects. J Thromb Haemost. 2011; 9 Suppl 1: 44-55.

17. Manne BK, Badolia R, Dangelmaier C, et al. Distinct pathways regulate Syk protein activation downstream of immune tyrosine activation motif (ITAM) and hemITAM receptors in platelets. J Biol Chem. 2015; 290: 11557-68.

18. Watson SP, Herbert JM, Pollitt AY. GPVI and CLEC-2 in hemostasis and vascular integrity. J Thromb Haemost. 2010; 8: 1456-67.

19. Nieswandt B, Varga-Szabo D, Elvers M. Integrins in platelet activation. J Thromb Haemost. 2009; 7 Suppl 1: 206-9. 
20. Shattil SJ, Kim C, Ginsberg MH. The final steps of integrin activation: the end game. Nat Rev Mol Cell Biol. 2010; 11: 288-300.

21. Huang J, Li X, Shi X, et al. Platelet integrin $\alpha$ llb $\beta 3$ : signal transduction, regulation, and its therapeutic targeting. J Hematol Oncol. 2019; 12: 26.

22. Burkhart JM, Vaudel M, Gambaryan S, et al. The first comprehensive and quantitative analysis of human platelet protein composition allows the comparative analysis of structural and functional pathways. Blood. 2012; 120: e73-82.

23. Cosemans JMEM, Iserbyt BF, Deckmyn H, Heemskerk JWM. Multiple ways to switch platelet integrins on and off. J Thromb Haemost. 2008; 6: 1253-61.

24. Watson SP, Auger JM, McCarty OJ, Pearce AC. GPVI and integrin alphallb beta3 signaling in platelets. J Thromb Haemost. 2005; 3: 1752-62.

25. Swieringa F, Kuijpers MJ, Heemskerk JW, van der Meijden PE. Targeting platelet receptor function in thrombus formation: the risk of bleeding. Blood Rev. 2014; 28: 9-21.

26. Samaha FF, Kahn ML. Novel platelet and vascular roles for immunoreceptor signaling. Arterioscler Thromb Vasc Biol. 2006; 26: 2588-93.

27. Abdul Sater H. Receptor tyrosine kinases in human platelets: A review of expression, function and inhibition in relation to the risk of bleeding or thrombocytopenia from phase I through phase III trials. J Cancer Prev Curr Res. 2017; 8.

28. Kuijpers MJE, Schulte V, Bergmeier W, et al. Complementary roles of glycoprotein VI and alpha2beta1 integrin in collagen-induced thrombus formation in flowing whole blood ex vivo. Faseb j. 2003; 17: 685-7.

29. Kuijpers MJE, Pozgajova M, Cosemans JMEM, et al. Role of murine integrin alpha2beta1 in thrombus stabilization and embolization: contribution of thromboxane A2. Thromb Haemost. 2007; 98: 107280.

30. Grüner S, Prostredna M, Schulte V, et al. Multiple integrin-ligand interactions synergize in shearresistant platelet adhesion at sites of arterial injury in vivo. Blood. 2003; 102: 4021-7.

31. Chang JC, Chang HH, Lin CT, Lo SJ. The integrin alpha6beta1 modulation of PI3K and Cdc42 activities induces dynamic filopodium formation in human platelets. J Biomed Sci. 2005; 12: 881-98.

32. Trzaskowski B, Latek D, Yuan S, Ghoshdastider U, Debinski A, Filipek S. Action of molecular switches in GPCRs-theoretical and experimental studies. Curr Med Chem. 2012; 19: 1090-109.

33. Offermanns S. Activation of platelet function through $\mathrm{G}$ protein-coupled receptors. Circ Res. 2006; 99: 1293-304.

34. Gachet C. P2 receptors, platelet function and pharmacological implications. Thromb Haemost. 2008; 99: 466-72.

35. Noé L, Peeters K, Izzi B, Van Geet C, Freson K. Regulators of platelet cAMP levels: clinical and therapeutic implications. Curr Med Chem. 2010; 17: 2897-905.

36. Law LA, Graham DK, Di Paola J, Branchford BR. GAS6/TAM pathway signaling in hemostasis and thrombosis. Front Med (Lausanne). 2018; 5: 137.

37. Lemke G. Biology of the TAM receptors. Cold Spring Harb Perspect Biol. 2013; 5: a009076.

38. Cosemans JMEM, Van Kruchten R, Olieslagers S, et al. Potentiating role of Gas6 and Tyro3, Axl and Mer (TAM) receptors in human and murine platelet activation and thrombus stabilization. J Thromb Haemost. 2010; 8: 1797-808.

39. Zhou J, Yang A, Wang Y, et al. Tyro3, Axl, and Mertk receptors differentially participate in platelet activation and thrombus formation. Cell Commun Signal. 2018; 16: 98.

40. Abrantes JL, Tornatore TF, Pelizzaro-Rocha KJ, et al. Crosstalk between kinases, phosphatases and miRNAs in cancer. Biochimie. 2014; 107 Pt B: 167-87.

41. Feneyrolles $C$, Spenlinhauer A, Guiet $L$, et al. Axl kinase as a key target for oncology: focus on small molecule inhibitors. Mol Cancer Ther. 2014; 13: 2141-8.

42. Torii T, Yamauchi J. Gas6-Tyro3 signaling is required for Schwann cell myelination and possible remyelination. Neural Regen Res. 2016; 11: 215-6.

43. Verma A, Warner SL, Vankayalapati H, Bearss DJ, Sharma S. Targeting Axl and Mer kinases in cancer. Mol Cancer Ther. 2011; 10: 1763-73.

44. Franco AT, Corken A, Ware J. Platelets at the interface of thrombosis, inflammation, and cancer. Blood. 2015; 126: 582-8. 
45. Sabrkhany S, Griffioen AW, Oude Egbrink MG. The role of blood platelets in tumor angiogenesis. Biochim Biophys Acta. 2011; 1815: 189-96.

46. De Stefano V. Arterial thrombosis and cancer: the neglected side of the coin of Trousseau syndrome. Haematologica. 2018; 103: 1419-21.

47. Navi BB, Reiner AS, Kamel H, et al. Risk of arterial thromboembolism in patients with cancer. J Am Coll Cardiol. 2017; 70: 926-38.

48. Trousseau A. Plemasia alba dolens. In: The Sydenham Society (ed) Clinique Medicale de l'Hotel-Dieu de Paris. 1865; 3: 654-712.

49. van Es N, Sturk A, Middeldorp S, Nieuwland R. Effects of cancer on platelets. Seminars in Oncology. 2014; 41: 311-8.

50. Verheul HM, Hoekman K, Luykx-de Bakker S, et al. Platelet: transporter of vascular endothelial growth factor. Clin Cancer Res. 1997; 3: 2187-90.

51. Klement GL, Yip TT, Cassiola F, et al. Platelets actively sequester angiogenesis regulators. Blood. 2009; 113: 2835-42.

52. Palacios-Acedo AL, Mège D, Crescence L, Dignat-George F, Dubois C, Panicot-Dubois L. Platelets, thrombo-inflammation, and cancer: Collaborating with the enemy. Front Immunol. 2019; 10: 1805.

53. Nierodzik ML, Karpatkin S. Thrombin induces tumor growth, metastasis, and angiogenesis: Evidence for a thrombin-regulated dormant tumor phenotype. Cancer Cell. 2006; 10: 355-62.

54. Wojtukiewicz MZ, Sierko E, Hempel D, Tucker SC, Honn KV. Platelets and cancer angiogenesis nexus. Cancer Metastasis Rev. 2017; 36: 249-62.

55. Wang S, Li Z, Xu R. Human cancer and platelet interaction, a potential therapeutic target. Int J Mol Sci. 2018; 19 .

56. Nieswandt B, Hafner M, Echtenacher B, Männel DN. Lysis of tumor cells by natural killer cells in mice is impeded by platelets. Cancer Res. 1999; 59: 1295-300.

57. Li N. Platelets in cancer metastasis: To help the "villain" to do evil. Int J Cancer. 2016; 138: 2078-87.

58. Egan K, Cooke N, Kenny D. Living in shear: platelets protect cancer cells from shear induced damage. Clin Exp Metastasis. 2014; 31: 697-704.

59. Schlesinger M. Role of platelets and platelet receptors in cancer metastasis. J Hematol Oncol. 2018; 11: 125 

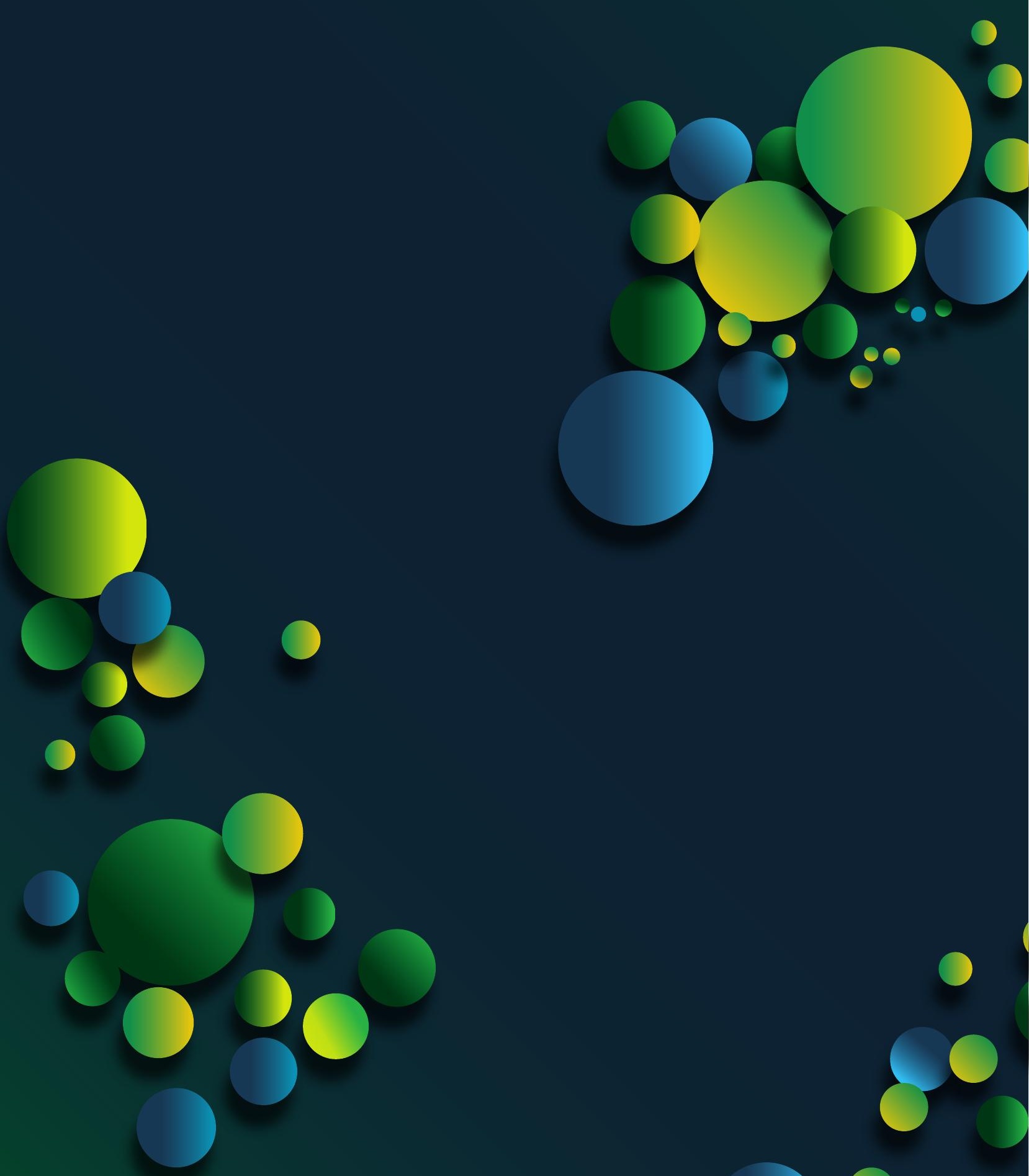


\section{Chapter 2}

Acquired platelet antagonism: off-target antiplatelet effects of malignancy treatment with tyrosine kinase inhibitors

Tullemans BME, Heemskerk JWM and Kuijpers MJE

J Thromb Haemost 2018; 16:1686-1699

Reprinted with permission 


\section{Abstract}

Platelets can contribute to tumour progression and metastasis. Cancer patients are at increased risk of thrombosis, while advanced stages of cancer associate with thrombocytosis or increased platelet reactivity. Tyrosine kinase inhibitors (TKIS) are widely used as a targeted strategy for cancer treatment, aiming to prolong progression-free survival of the patients. Because of their broad kinase target spectrum, most TKIs inevitably have off-target effects. Platelets rely on on tyrosine kinase activity for their activation. Frequently observed side effects are lowering of platelet count and inhibition of platelet functions, whether or not accompanied by an increased bleeding risk. In this review we aim to give insight into: (i) 38 TKIs that are currently used for treatment of different types of cancer, either on the market or in clinical trials, (ii) how distinct TKIs can inhibit activation mechanisms in platelets, and (iii) the clinical consequences of the antiplatelet effects due to TKI treatment. For several TKIs, the knowledge on affinity for their targets does not align with published effects on platelets and reported bleeding events. Together, this review should raise awareness of the potential antiplatelet effects of several TKIs, which will be enhanced in the presence of antithrombotic drugs. 


\section{Introduction}

Human blood platelets are required for haemostasis, and also contribute to pathological thrombosis ${ }^{1}$. Platelets normally circulate in the blood at a concentration of $150-450 \times 10^{9} / \mathrm{L}$, with a life span of around 10 days. Thrombocytopenia, a low platelet concentration which increases the bleeding risks, is defined as a count $<50 \times 10^{9} / \mathrm{L}$. By implication, only a fraction of the normally circulating platelets seems to be required for proper haemostasis, as could be demonstrated in mice $^{2}$. Hence, the majority of circulating platelets are likely to contribute to other physiological processes, which can include maintenance of vascular integrity, tissue repair, immune responses, and infection prevention. The existence of different populations of platelets, primed by their environment ${ }^{3}$, may even suggest a certain degree of specialisation in these functions. Considering the 'overload' of circulating platelets, it is not a surprise that these a-nucleated cells also contribute to pathological processes, including cancer.

It is well known that cancer patients are at increased risk for thrombosis ${ }^{4}$, and that advanced stages of cancer are associated with increased platelet reactivity ${ }^{5}$. Recent papers furthermore suggest that the growth factor composition and even the RNA profile of platelets can change due to the presence of cancer ${ }^{6,7}$. On top of this, malignancy can lead to thrombocytosis (elevated platelet count), which is regarded as a negative predictor of survival ${ }^{8}$. The suggested mechanism is enhanced platelet formation by megakaryocytes, due to tumour-derived interleukin-6 production and high thrombopoietin production in the liver ${ }^{9}$. This points to a cycle of tumour-induced platelet formation and activation, followed by growth factor release and tumour promotion and metastasis. This reciprocal interaction between platelet activation and tumour growth has recently been reviewed by others ${ }^{10}$. The model proposed in 2000 is that tumours secrete chemokines which recruit blood cells, including platelets, and thus promote the process of angiogenesis, i.e., the formation of new blood vessels from pre-existing vessels, in order to secure tumour development and metastasis ${ }^{11}$. Platelets contain numerous pro-angiogenic (next to antiangiogenic) molecules in their $\alpha$-granules, which are either synthesised by megakaryocytes in the bone marrow or are taken up via endocytosis ${ }^{12}$.

In the majority of cancers, oncogene-encoded proteins or growth factor receptors are amplified or mutated, which carry tyrosine protein kinase activity or steer both tyrosine and serine/threonine kinases in the downstream signalling pathways. Accordingly, abnormal protein kinase activity is considered as a hallmark of tumour biology ${ }^{13}$, resulting in altered cell proliferation, survival, motility, metabolism, angiogenesis, and evasion of immune responses ${ }^{14}$. This insight has prompted the search for tyrosine kinase inhibitors (TKIs) as targeted therapeutic drugs in oncology.

Protein tyrosine kinases are classified into the receptor-linked tyrosine kinases, e.g., the vascular endothelial growth factor (VEGF) and platelet-derived growth factor (PDGF) receptors; and the cytosolic non-receptor kinases, such as Src family kinases (SFK), Syk and Btk. In tumours, 
often gain-of-function mutations are seen in the genes encoding for tyrosine kinases, resulting in constitutively active proteins. Examples of such aberrant oncoproteins are BCR-ABL and NPMALK. The latter are fusion genes that originate from reciprocal translocation of genetic material between two chromosomes, resulting in proteins that are 'always on' and leading to uncontrolled cell division. In the past fifteen years almost 40 TKIs have been developed and approved for cancer treatment (Table 2.1). In most cases, these are small molecules with relatively high affinity to the so-called targeted kinases, but often with similar affinities to other tyrosine kinases. As many of these tyrosine kinases are also expressed in non-proliferative cells, including platelets, TKIs clearly can have relevant off-target effects.

Blood platelets are unable to proliferate or differentiate but display high tyrosine kinase activities in comparison to other cell types. In platelet activation, tyrosine phosphorylation is one of the key signal transduction mechanisms. As described below, non-receptor tyrosine kinases are activated by the collagen receptor, glycoprotein (GP)VI; the C-type lectin-like receptor 2 (CLEC2 ); the von Willebrand factor (VWF) receptor, GPIb-IX-V; and by adhesion-regulating integrins ${ }^{15}$, 16. In this review we aim to give insight into: (i) the TKIs that are currently used for treatment of different types of cancer, either on the market or in clinical trials, (ii) how distinct TKIs can inhibit activation mechanisms in platelets, and (iii) the clinical consequences of the antiplatelet effects due to TKI treatment.

\section{Cancer treatment with tyrosine kinase inhibitors}

TKIs are defined as pharmaceutical drugs that inhibit tyrosine kinases. The TKIs that are currently in use are in majority small molecules that can pass the cell membrane. Their common mode of action is by competition with ATP in the conserved catalytic binding site in the superfamily of (non)receptor tyrosine kinases. Because of the comparable structure of the catalytic pocket of tyrosine kinases, TKIs often target multiple kinases that play a role in several signalling pathways. At present, around 40 drugs with TKI activity have been approved by the USA Food and Drug Administration or have entered clinical trials for anticancer treatment (Table 2.1).

Based on their precise action, TKIs can be classified into four categories ${ }^{17}$. Type I inhibitors target the active conformation of the kinase and compete with the ATP-binding site (example: sunitinib). Type II inhibitors instead recognise the inactive conformation, and thereby indirectly compete with ATP by occupying a hydrophobic pocket near the ATP-binding site. Accordingly, type II inhibitors are considered to modify the kinase activity in an allosteric manner (examples are imatinib and sorafenib). Type III compounds are the so-called allosteric inhibitors. These bind more distantly from the ATP-binding site and inhibit kinase activity via classical allosteric interference (examples are MEK1 inhibitors such as selumetinib). Type IV inhibitors are also known as covalent inhibitors, forming an irreversible covalent bond near the active site of the kinase, usually by reacting with a cysteine residue. This blocks the binding of ATP and prevents activation of the 
kinase (examples are ibrutinib and vantedanib).

Although directed against specific targets, almost all TKIs affect multiple kinases. Table 2.1 provides an overview of the currently used TKIs that are prescribed for which cancer type, together with their target kinases in tumour cells. The majority of the drugs are targeted at receptor tyrosine kinases for growth factors (EGF, FGF, PDGF, VEGF) or for differentiation/proliferation factors (Flt, Fms, Kit, Ret). Several of the drugs (in addition) target intracellular tyrosine kinases (ABL, B-Raf, Btk, Itk, MEK, SFK, Syk). Although affinities towards individual kinases will differ, essentially all TKIs have a more or less broad efficacy, which in some cases is intentional. For instance, both VEGF and PDGF are known to be important in tumour angiogenesis. Sunitinib, blocking signalling via both types of receptors, thus will have a broader action spectrum than vatalanib, targeting only VEGF receptors ${ }^{18}$. In general, the use of TKIs for treatment of specific tumours depends on the affinity for the targeted kinases ${ }^{19}$ and the pharmacokinetics at indicated dosages and treatment regimens ${ }^{20}$ (Table 2.1). As far as understood, the ultimate specificity is not strongly correlated with chemical structure or the subfamily of the targeted kinase ${ }^{18}$.

Receptor-linked tyrosine kinases are also abundantly expressed in non-tumour cells, and treatment with TKIs will inevitably have off-target effects, thus interfering with the normal function of non-diseased cells and tissues. This can explain side effects of treatment, varying from general complications as fatigue, diarrhoea, and nausea, to specific complications like hand-foot syndrome ${ }^{21}$. In spite of drug-to-drug variations, many TKIs can cause skin toxicity, even in $>50 \%$ of patients ${ }^{22}$. For antiangiogenic TKIs, 'blood-related' side effects have been reported, such as hypertension, myelosuppression and bleeding ${ }^{23}$. Incidentally, also on-treatment cardiovascular events have been reported, for instance linked to affected vascular integrity of the endothelium ${ }^{24}$. TKI effects on the vessel wall comprise endothelial dysfunction and increased capillary leakage ${ }^{25}$, which may contribute to an increased bleeding tendency. Paradoxically, interfering with the integrity of endothelial cells can shift the haemostatic balance in favour of thrombosis, which might explain why treatment with TKIs can associate with arterial thromboembolic events in cancer patients ${ }^{25,26}$.

Despite clinical benefit of the prescribed TKIs, some of the patients who initially respond to the therapy experience a relapse, e.g., due to acquired drug resistance of the tumour ${ }^{14}$. In such cases, the treatment schedule is adjusted, or a switch is made to an alternative TKI as a secondor third-line treatment (Table 2.1). In specific cases, several TKIs can be combined for effective blockade of one or two signalling pathways ${ }^{14,21}$. Furthermore, chemotherapy or radiation therapy can be complemented with TKI treatment ${ }^{13}$.

\section{Protein tyrosine kinases implicated in platelet activation}

A global overview of platelet tyrosine kinases signalling underneath relevant receptors, as well as the downstream platelet responses is given in Table 2.2. 


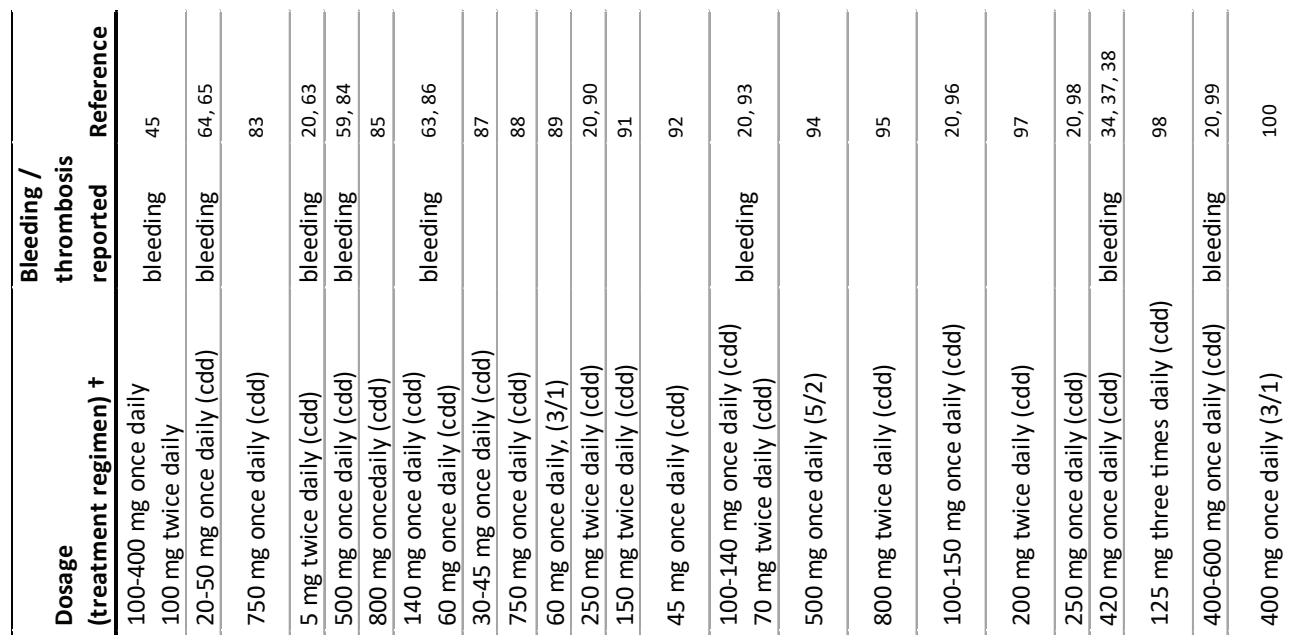

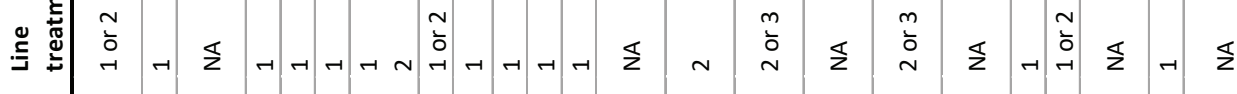




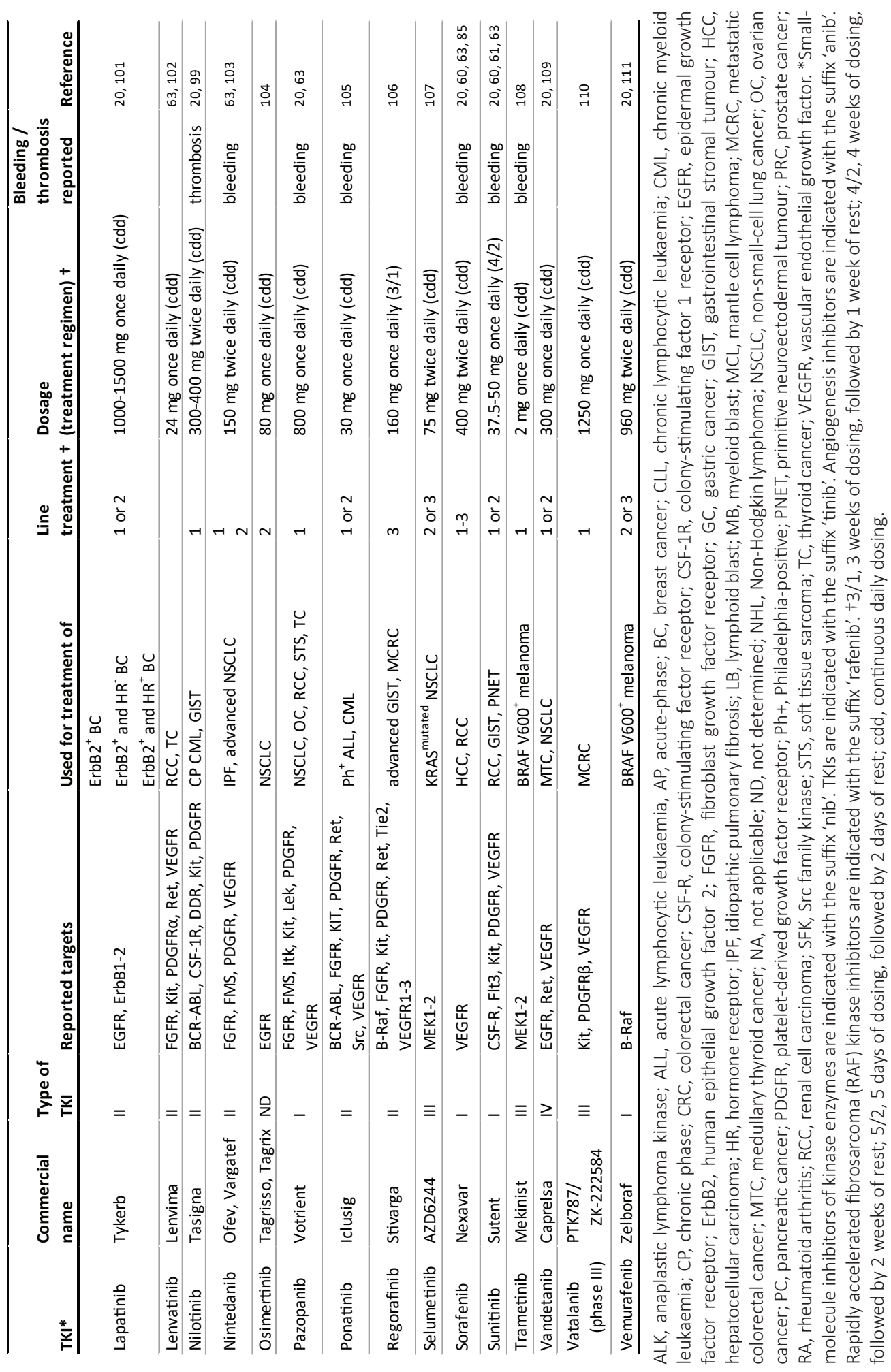


GPVI signalling. Collagen-induced platelet activation is established via the tyrosine kinase-linked receptor GPVI, a member of the immunoglobulin superfamily. GPVI is linked to the Fc receptor $\gamma(F c R \gamma)$ chain, which contains two immunoreceptor tyrosine-based activation motives (ITAM) requiring phosphorylation to mediate platelet activation ${ }^{27}$. Ligand binding and dimerization of the GPVI-FcRy complex leads to activation of the SFK isoforms Src, Fyn and Lyn, which in turn phosphorylate the FcRy ITAM tyrosine residues to recruit and phosphorylate Syk ${ }^{1,}$ 28. The tyrosine kinases SFK and Syk furthermore phosphorylate downstream targets, including the transmembrane adapter linker for activated T cells (LAT) and the Src homology 2 domaincontaining leukocyte phosphoprotein (SLP76). The consequence is the formation of a large signalling complex, including LAT, SLP76, Btk, isoforms of phosphoinositide-3 kinase and Tec family kinases ${ }^{29}$. A key downstream event is the phosphorylation and activation of the second messenger-generating phospholipase $(\mathrm{PLC}) \mathrm{\gamma} 2$, resulting in $\mathrm{Ca}^{2+}$ mobilisation and protein kinase $C$ (PKC) activity. Further responses are integrin activation, thromboxane $A_{2}$ release, granule secretion and phosphatidylserine exposure.

CLEC-2 signalling. A similar powerful activation pathway of platelets is induced via CLEC-2, a C-type lectin receptor also acting through tyrosine phosphorylation. Known ligands of this receptor are podoplanin (expressed in tumour tissue among others) and the snake venom rhodocytin. Signalling occurs through a so-called hemITAM motif ${ }^{30}$. The clustering of CLEC-2 induces more or less similar events as described for GPVI. Starting with the tyrosine phosphorylation of SFK and Syk, a signalling complex is formed including Tec family tyrosine kinases, with as result PLCY2 and PI3K activation. Consequences are, again, integrin activation, granule release, and thromboxane $\mathrm{A}_{2}$ production $^{30}$.

GPIb-IX-V signalling. Interaction of VWF with the GPIb-IX-V receptor is one of the first steps in platelet tethering and adhesion under shear flow ${ }^{1}$. This interaction causes only weak signalling, e.g., leading to restructuring of the actin cytoskeleton, with under certain conditions phosphorylation of SFK (SrC, Fyn, Lyn) and activation of PI3K isoforms ${ }^{1}$. Integrin $\alpha_{111} \beta_{3}$ activation and platelet spreading are a result of this.

Table 2.2. Protein tyrosine kinases implicated in platelet activation responses: global overview of tyrosine kinases implicated in signaling via key platelet receptors, as well as downstream platelet responses. Summarized from ${ }^{1,16,18,30}$

\begin{tabular}{llll}
\hline \multirow{2}{*}{ Receptor } & \multicolumn{2}{l}{ Signalling tyrosine kinases } & Platelet response \\
\cline { 2 - 4 } \cline { 3 - 4 } & SFK & Other & $\begin{array}{c}\mathrm{Ca}^{2+} \text { mobilisation, integrin activation, } \\
\text { degranulation }\end{array}$ \\
\hline CLEC-2 & Src, Fyn, Lyn, Fgr & Btk, Syk, Tec & $\begin{array}{c}\mathrm{Ca}^{2+} \text { mobilisation, integrin activation, } \\
\text { degranulation }\end{array}$ \\
\hline GPIb-IX-V & Src, Fyn, Lyn & Btk & Integrin activation \\
\hline Integrin $\alpha_{I I b} \beta_{3}$ & Src, Fyn, Lyn & FAK, Syk, SLP76, Pyk2 & $\begin{array}{l}\text { Spreading, outside-in signaling, } \\
\text { clot retraction }\end{array}$ \\
\hline
\end{tabular}

CLEC-2, C-type lectin-like receptor 2; GP, glycoprotein; SFK, Src family kinase; SLP76, Src homology 2 domain-containing leukocyte phosphoprotein 
Integrin-dependent signalling. Platelet integrins, in particular $\alpha_{11 b} \beta_{3}, \alpha_{2} \beta_{1}$ and $\alpha \vee \beta_{3}$, regulate adhesion, aggregation and thrombus formation ${ }^{28}$. Especially regarding integrin $\alpha_{\| 1 b} \beta_{3}$ (ligands: fibrinogen, VWF and other matrix proteins) much research has been performed to the outside-in signalling events triggered by the occupied, activated conformation. Several tyrosine kinases are implicated in this signalling pathway, including FAK, Pyk2, Src, SLP76 and Syk ${ }^{29,} 31$.

\section{Effects of TKIs on platelet function}

Tyrosine kinases are targeted by TKIs as treatment for cancer, where their most important effect is the prolongation of progression free survival. Given the presence of on-target or related off-target tyrosine kinases in megakaryocytes and platelets, it is to be expected that several TKIs interfere with platelet formation and/or platelet activation processes.

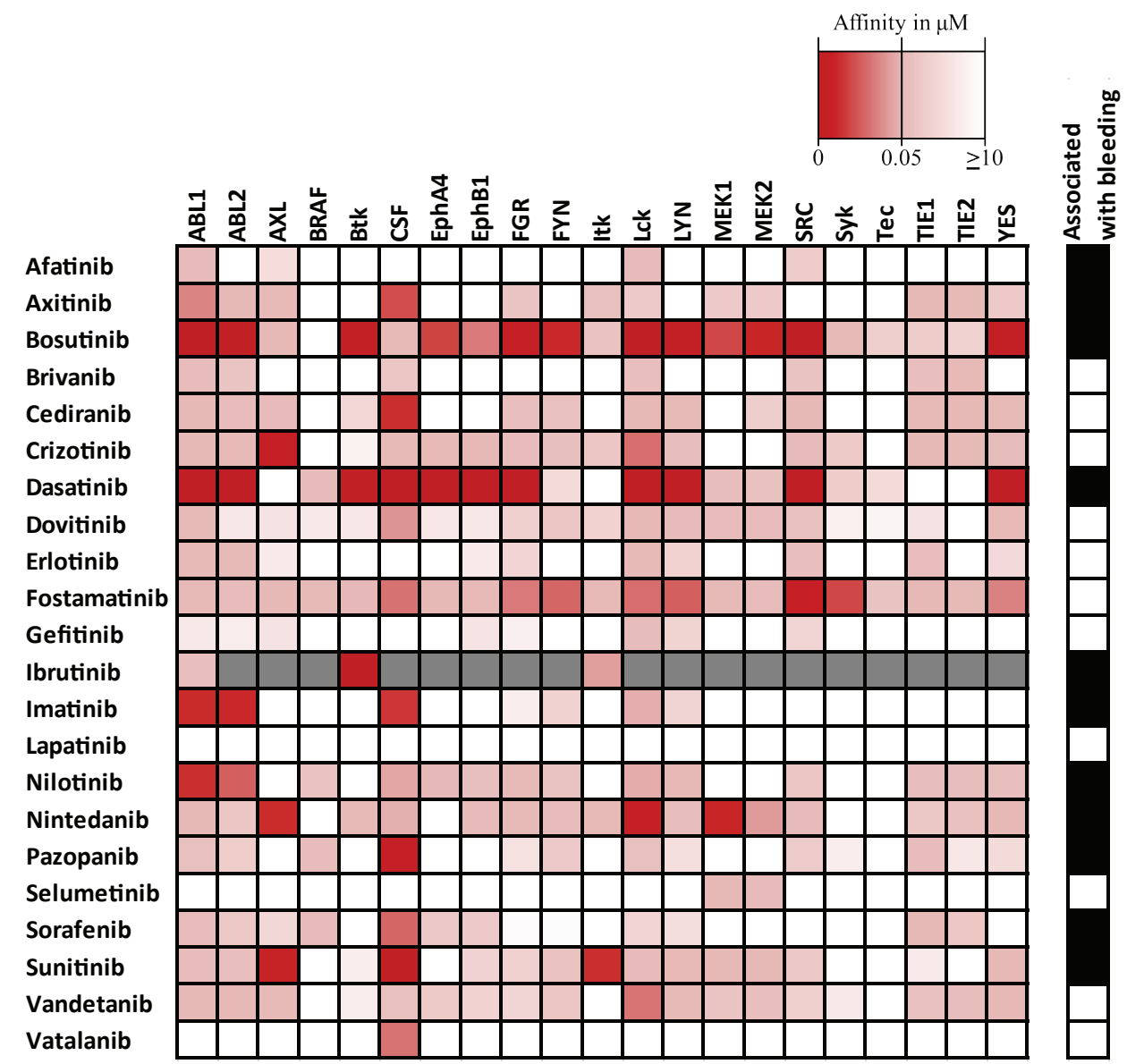

Figure 2.1. Heatmap of affinity profile of tyrosine kinase inhibitors (TKIs) with described targets that are known to be expressed in platelets. Shown are normalized affinity-based dissociation constants $\left(K_{d}\right)$ of the indicated TKIs determined for purified kinases ${ }^{18,19,33,36}$, as far as they are present in platelets. Scaling is from 0 to 10 , with the lowest $K_{d}$ indicating the highest affinity (deep red is highest affinity = lowest $\mathrm{K}_{\mathrm{d}}$; white is lowest affinity = highest $\mathrm{K}_{\mathrm{d}}$; pink gradient represents intermediate $\mathrm{K}_{d}^{\prime} \mathrm{S}$; gray values have not been described). The right column indicates increased bleeding risk (black, yes; white, no or unknown); see Table 2.1. 


\section{Chapter 2}

To provide an overview, we evaluated the inhibition profiles of TKIs (22 in total) against target protein tyrosine kinases that are known to be expressed in platelets ${ }^{32}$. Markedly, the majority of these TKIs demonstrated relatively low affinities for kinases with crucial roles in platelet activation processes (SFK, Syk, Btk, MEK and Eph isoforms), with as exceptions bosutinib, dasatinib, fostamatinib, nintedanib and sunitinib (Figure 2.1). For such an in vitro affinity-based analysis ${ }^{18,19,33}$, it should be realised that the presence of blood plasma and blood cells can profoundly change the bioavailability of a TKI, apart from its metabolic and pharmacokinetic profile. Markedly, bleeding symptoms have been reported for 11 of these TKIs. For individual TKIs, based on their known targets, we summarised in Table 2.3 which of these targets are expressed in platelets. Furthermore, we made an inventory of the published effects of these TKIs on platelet responses.

Ibrutinib is a covalently acting TKI that is targeted at the family members Btk and Tec, which probably explains the strong effects observed on platelets ${ }^{34}$. It is frequently used for the treatment of mantle cell lymphoma (MCL) and chronic lymphocytic leukaemia (CLL). Emerging data suggest that ibrutinib could also be used to treat solid tumours ${ }^{35}$ and has been shown to inhibit several other kinases such as Itk, JAK3, Hck, Blk, EGFR, ErbB2 and ErbB4 ${ }^{36}$. Ibrutinib treatment is associated with a risk of bleeding ${ }^{34,37,38}$. Being the most investigated TKI with regard to platelets, ibrutinib has been demonstrated to potently inhibit collagen-induced responses of platelets from patients on-treatment ${ }^{39-44}$. Efficient inhibition by ibrutinib of the GPVI pathway (in response to collagen or collagen-related peptide) has been demonstrated to proceed via reduced PLCY2 phosphorylation ${ }^{42}$. In treated patients, the suppression of collagen-induced platelet aggregation correlated with the occurrence of bleeding events ${ }^{42}$. Subsequent studies showed that ibrutinib suppressed multiple (mostly) GPVI-dependent platelet responses, including adhesion, spreading, calcium fluxes, secretion, phosphatidylserine exposure and clot retraction ${ }^{40,}$ 43. In addition, evidence was obtained for reduced $\alpha_{\| 1 \mathrm{~b}} \beta_{3}$-dependent outside-in signalling, linked to thrombus instability in vitro ${ }^{40}$. Several studies confirmed that Btk can act as a central target of ibrutinib in GPVI-stimulated platelets, although downstream tyrosine kinases may be affected as well ${ }^{40,42,44}$. In vitro, ibrutinib was found to fully inhibit the tyrosine phosphorylation of Src and PLCY2 ${ }^{42}$. Other affected events were the phosphorylation of Fyn, Lyn, Btk, Tec and Syk ${ }^{40,44}$. Taken together, this pointed to a potent suppression of ibrutinib of the GPVI signalosome with as major target the Tec family kinases.

The second-generation drug, acalabrutinib, is a more selective, irreversible Btk inhibitor. In a clinical trial with patients with relapsed CLL, acalabrutinib showed promising safety and efficacy profiles, although some minor bleedings were still reported ${ }^{45}$. A recent study comparing treatment with ibrutinib or acalabrutinib indicated that both compounds impaired the platelet aggregation responses after collagen receptor stimulation ${ }^{46}$. Both drugs inhibited platelet Btk and Tec at physiological concentrations, while only ibrutinib inhibited SFK isoforms. This provided 


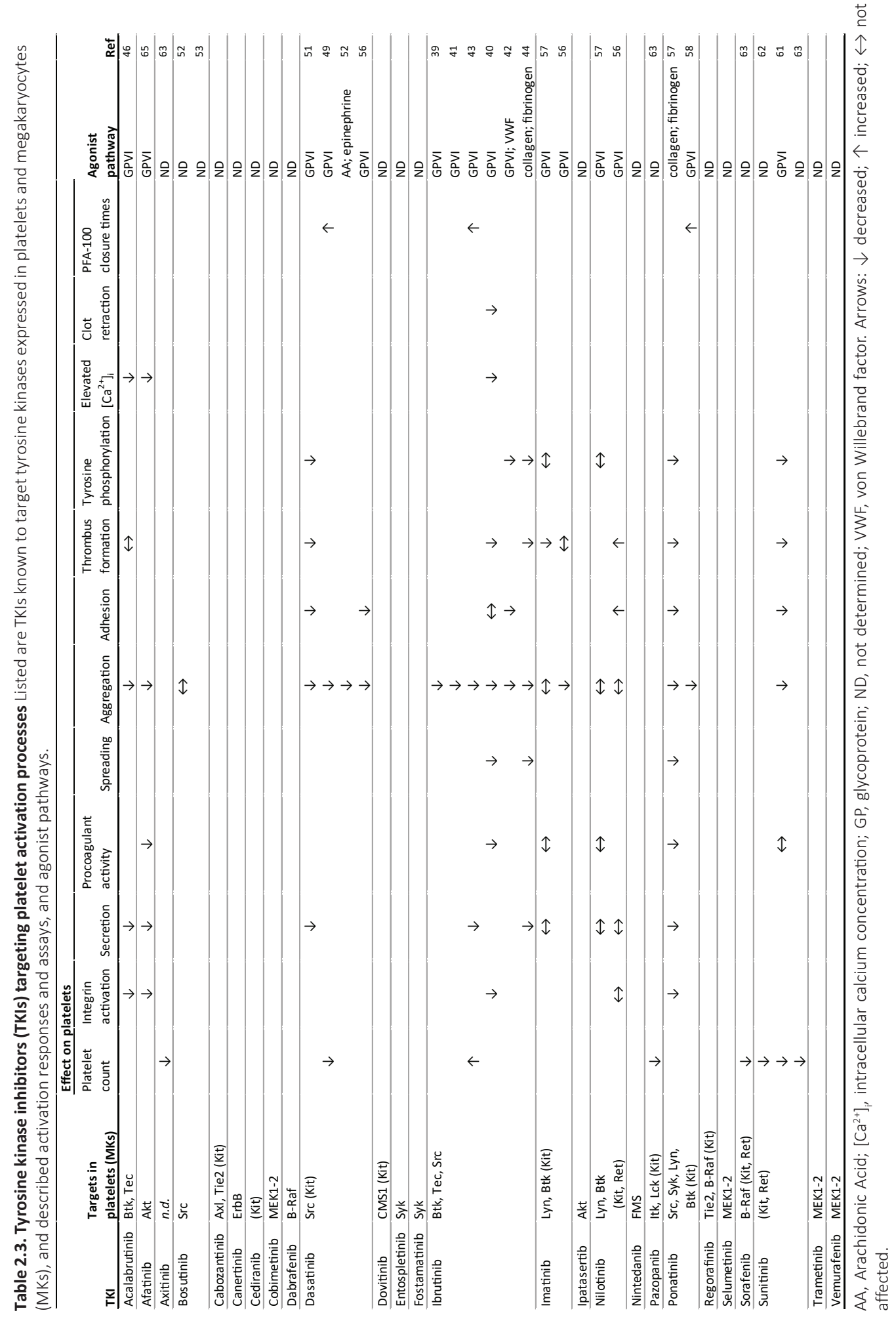




\section{Chapter 2}

an explanation why only ibrutinib caused dysfunctional thrombus formation, in contrast to acalabrutinib ${ }^{46}$.

Imatinib, targeting the oncogenic kinase $B C R-A B L$, was the first tyrosine kinase inhibitor developed for chronic myeloid leukaemia (CML). Imatinib is known to block Kit and PDGF receptors, as well as Erk and Akt isoforms ${ }^{47}$. This TKI has been reported to evoke thrombocytopenia in $18 \%$ of the treated patients, with haemorrhages being reported as well ${ }^{48}$. Despite a high efficacy of imatinib, 15\% of patients appear to develop clinical resistance and relapse after initial response to therapy.

Dasatinib and nilotinib have been developed as second generation BCR-ABL inhibitors for the treatment of CML. Two case reports showed that dasatinib could induce a bleeding diathesis correlated to platelet dysfunction, which was reversed upon cessation of the dasatinib intake ${ }^{49}$, 50. Studies with blood from patients or healthy subjects showed an impairment of platelet aggregation by dasatinib given in vivo or in vitro ${ }^{51,52}$. In particular, platelet aggregation in response to collagen, arachidonic acid and epinephrine was affected ${ }^{52}$, along with collagen-induced thrombus formation ${ }^{51,53}$, thus suggesting interference with GPVI signalling and other pathways. The mechanism of dasatinib-induced inhibition in platelets was elucidated from changes in the protein phosphorylation profile after collagen stimulation. Herein, dasatinib appeared to interfere with SFK, PLC and PI3K activities ${ }^{51}$. Thrombocytopenia is a relatively common side effect of dasatinib treatment ${ }^{54}$. This is proposed to be due to reduced megakaryocytopoiesis, thus resulting in lower platelet production ${ }^{55}$. The combined quantitative (via megakaryocytes) and qualitative (via signalling) platelet dysfunction most likely is the reason of the relatively high bleeding incidence in dasatinib-treated patients. Compared to dasatinib, imatinib induces less antiplatelet activity and causes less bleeding at clinically achievable doses, likely due to the fact that it is less inhibitory for SFK isoforms. On the other hand, nilotinib is reported to potentiate thrombus formation and increase the risk of thrombosis ${ }^{56}$.

Ponatinib and bosutinib are third generation BRC-ABL inhibitors for the treatment of $C M L$, which are applicable when patients develop resistance to imatinib. The use of ponatinib has been associated with bleeding diatheses, along with changes in platelet count and function, which thus may explain the impaired haemostasis ${ }^{57,58}$. One study describes inhibiting effects of ponatinib, imatinib and nilotinib on platelet activation, secretion, and aggregation in response to collagen ${ }^{57}$. Herein, ponatinib appeared to be the most potent platelet inhibitor. Ponatinib increased PFA-100 closure times, likely by suppressing ITAM signalling via several tyrosine kinases, including Src, Lyn, Syk, and Btk ${ }^{57,58}$. In contrast, imatinib and nilotinib did not affect platelet granule secretion, procoagulant activity or aggregation, and only moderately reduced the tyrosine phosphorylation of Lyn and Btk, as compared to ponatinib ${ }^{57}$. Yet, whole blood thrombus formation was reduced by imatinib and ponatinib to a similar extent. For bosutinib (also targeting Src), bleeding has been reported as well ${ }^{59}$, although in patients it did not affect platelet aggregation in response to 
several agonists ${ }^{52}$. In microfluidic whole blood testing of platelet thrombus formation, bosutinib was found to be less potent than dasatinib ${ }^{53}$. Overall, it appears that BCR-ABL inhibitors used for leukaemia treatment have an inhibiting effect on platelet functions, with the possible exception of nilotinib.

Sunitinib, sorafenib and pazopanib are multi-target TKIs used for the treatment of renal cell carcinoma, aiming to inhibit tumour angiogenesis via interference with the receptors for VEGF and PDGF. Such multi-target TKIs may potentially also inhibit tyrosine kinases present in platelets. For sunitinib and sorafenib, a meta-analysis of 23 trials (>6,500 patients) showed an increased incidence of bleeding events with $16.7 \%$ on all grades of bleeding, and a $2.4 \%$ probability of high-grade bleeding events ${ }^{60}$. For sunitinib, both quantitative and qualitative effects on platelets are reported. The compound can be taken up by platelets from healthy donors and renal cell carcinoma patients, and hence reduce the platelet functionality in a way depending on protein tyrosine phosphorylation ${ }^{61}$. In patients on-treatment, this is accompanied by a decrease in platelet count $^{61,62}$. For sorafenib, similarly to other TKIs used for treatment of renal cell carcinoma such as pazopanib, an increased risk of bleeding has also been observed ${ }^{63}$, but effects on platelet function have not been investigated.

Afatinib is used for the first-line treatment of particularly patients with non-small cell lung cancers $^{64}$. It acts as an irreversible inhibitor of EGF and HER receptors. An increased bleeding risk of patients receiving afatinib has been linked to impaired platelet function and apoptosis ${ }^{65}$. In particular, afatinib impaired GPVI-induced platelet responses, including calcium fluxes, integrin activation, secretion, procoagulant activity and platelet aggregation (Table 2.3). On the other hand, thrombin-induced platelet responses were less strongly inhibited. Further investigation showed that the collagen-induced phosphorylation of Akt was decreased by afatinib ${ }^{65}$.

Cabozantinib, cediranib, dovitinib, lenvatinib, regorafinib and other TKIs co-target the receptor kinase Kit, which acts as a receptor for stem cell factor in megakaryocytopoiesis, regulating platelet production. The Kit receptor is known to signal via SFK, PI3K, Ras/MEK and JAK/STAT pathways ${ }^{66}$. In an analogous way, the thrombopoietin receptor Mpl signals via JAK2 and Tyk2 as immediate effector kinases, resulting in the activation of STAT isoforms ${ }^{67}$. For these signalling pathways, key tyrosine kinases are also present in platelets and/or megakaryocytes (Table 2.2 and 2.3), which raises the possibility that Kit-targeted TKIs can also modulate platelet function as well as platelet count. So far, the signalling effects of these TKIs on platelets have not been investigated in detail. Cabozantinib intake has been associated with a higher bleeding risk ${ }^{63}$. Related drugs are ruxolitinib, pacritinib and lesaurtinib, which also target JAK1/2 and are usually prescribed for myeloproliferative disorders, such as essential thrombocythemia, characterised by an elevated platelet count (thrombocytosis). The latter compounds appear to lower (normalise) the concentration of circulating platelets ${ }^{68}$, most likely via inhibition of the thrombopoietin pathway in megakaryocytes. However, effects on platelet function are still unclear. It is known that 
thrombopoietin potentiates agonist-induced platelet activation via JAK2 and Tyk2 signalling69. This indicates that this pathway is not strong enough to induce platelet activation on its own. Inhibition of JAK2 in platelets has been reported to only attenuate collagen-induced responses ${ }^{70}$.

For multiple prescribed TKIs with a broad target range, bleeding symptoms have not been reported (Table 2.1). Examples of these are fostamatinib, dovitinib and vantedanib, which based on their affinity profiles should affect SFK and/or MEK isoforms in platelets (Figure 2.1). In these cases, additional research is needed to verify effects on platelet count and activation, and to determine if there is a bleeding risk. Summarising the current knowledge of TKI effects on platelet activation responses, it appears that the majority of multi-target TKIs in particular suppresses GPVI-induced signalling pathways, but that they can also target kinase events downstream of other platelet receptors. As the inhibition of GPVI alone has been shown to prevent occlusive thrombus formation without causing bleeding ${ }^{71}$, it is most likely that the targeting of pathways downstream of additional receptors is responsible for an increased bleeding risk with these compounds.

\section{Clinical implications of platelet inhibition with TKIs}

Platelets are known to interact with cancer cells in the blood. Tumours exposed to the blood stream can activate platelets, while platelets can stimulate tumour angiogenesis, growth, and metastasis. The question has been raised whether and how interfering in the platelet-cancer loop via antiplatelet treatment may also influence cancer progression. Several studies have reported that aspirin or $\mathrm{P}_{2} \mathrm{Y}_{12}$ receptor inhibitors suppress tumour angiogenesis in vitro $^{72}$ and tumour growth in vivo ${ }^{73}$. A low daily aspirin intake has been associated with a reduced risk for the development of cancer as well as prevention of metastases ${ }^{74}$. The mechanism is not completely resolved, but it has been shown that inhibition of platelets by aspirin decreased their ability to stimulate cancer cell proliferation through modulation of the Myc oncoprotein ${ }^{75}$. For other antiplatelet drugs, clinical evidence of a beneficial effect on cancer is still lacking.

Of growing interest is the recent finding that platelets are capable to sequester bioactive compounds from the plasma, including growth factors ${ }^{12,76,77}$ and RNA species ${ }^{7}$. In addition, platelets accumulate the anticancer drugs bevacizumab ${ }^{78}$ and sunitinib (in granules) ${ }^{61}$. Other TKIs can also be taken up by platelets, which usually is a requirement for interaction with the intracellular kinases. Whether these TKIs can also secondarily be released by platelets and, perhaps, then contribute to tumour inhibition is matter of speculation. Another consideration is that uptake of TKIs by platelets (e.g., in patients with thrombocytosis) can reduce the availability of the compounds for tumour inhibition. On the other hand, it is likely that a decreased aggregation tendency of platelets (as induced by TKIs) also lowers tumour-induced platelet activation, and thereby reduces metastasis of tumour cells. However, the evidence for such a mechanism is at best indirect, and further investigation here is required. Taken together, it appears that platelets 
can interfere with drug effects in different ways and by different mechanisms, which should be taken into account upon therapeutic drug monitoring.

Application of the TKIs axitinib, dasatinib, pazopanib, sorafenib and sunitinib - all of which have indications may cause bleeding - can lead to a reduction of the platelet concentration in blood (Table 2.3). In cancer patients, thrombocytosis has been associated with a worse overall prognosis, when compared to patients with a normal platelet count ${ }^{8,9}$. In addition, in glioblastoma patients, who underwent concomitant radiotherapy and chemotherapy, a decrease in platelet count correlated with longer overall survival ${ }^{79}$. In renal cell carcinoma patients with metastasis, a reduction in platelet count after start of sunitinib treatment was associated with a better treatment outcome ${ }^{62}$. Although more research is needed, these studies suggest that platelet count can be used as one of the parameters to monitor anticancer treatment efficacy.

Besides platelet count, also platelet function can be affected by many TKIs, as indicated in Table 2.3. This suggests that combined platelet count and function tests could be used in clinical practice to assess the risk of bleeding upon TKI treatment. For the availability of such (point-ofcare) tests, we like to refer to the expert literature ${ }^{80}$. In the future it may be possible to use flowbased assays for this purpose. Our laboratory recently found that this method detects additive effects of low platelet count and impaired platelet functionality in patients with a bleeding phenotype $^{81}$.

It is known that cancer patients are at increased risk of thrombosis, also indicated as Trousseau's syndrome ${ }^{82}$. As a consequence, many cancer patients have a history of cardiovascular disease and are treated with antiplatelet or anticoagulant drugs. When additional treatment with a TKI is started, the clinical problem of an enhanced bleeding risk may arise. Another treatment issue is that some oral anticoagulants and TKIs (ibrutinib for instance) have a common metabolic pathway (CYP3A4), which can affect the pharmacokinetics of either drug. These interactions between TKIs and antithrombotic treatments need to be further investigated.

\section{Conclusions}

Several of the TKIs used for the treatment of cancer can increase the risk of bleeding. For some but not all of these, the bleeding tendency is linked to a lowering of platelet count and/or an impairment of platelet function. Based on their binding profiles, some other TKIs are predicated to have an antiplatelet effect, although no bleeding side effects have been reported so far. For a third group of TKIs, no published data on platelets are available. Given the suspected role of platelets in tumour progression, platelet inhibition may be a clinically relevant side-effect of several TKIs. Together, the available knowledge asks for awareness of the potential antiplatelet effects of TKIs, which are likely to be further enhanced in combination with antithrombotic drugs. 


\section{Acknowledgements}

This study was supported by the Maastricht Thrombosis Expertise Centre as part of the Heart and Vascular Centre (HVC) of the MUMC+.

\section{Author Contributions Statement}

BMET reviewed the literature, compiled tables and Fig. 2.1, and wrote the manuscript. JWMH was responsible for the outline, and wrote and revised the manuscript. MJEK was responsible for the outline, reviewed the literature, and wrote and revised the manuscript.

\section{Conflict of Interest Statement}

MJEK and JWMH report receiving grants from Pfizer during the conduct of the study. BMET states that she has no conflict of interest.

\section{References}

1. Versteeg HH, Heemskerk JW, Levi M, Reitsma PH. New fundamentals in hemostasis. Physiol Rev. 2013; 93: 327-58.

2. Morowski M, Vogtle T, Kraft P, Kleinschnitz C, Stoll G, Nieswandt B. Only severe thrombocytopenia results in bleeding and defective thrombus formation in mice. Blood. 2013; 121: 4938-47.

3. Baaten CCFMJ, Ten Cate H, van der Meijden PEJ, Heemskerk JWM. Platelet populations and priming in hematological diseases. Blood Rev. 2017; 31: 389-99.

4. Franco AT, Corken A, Ware J. Platelets at the interface of thrombosis, inflammation, and cancer. Blood. 2015; 126: 582-8.

5. Cooke NM, Egan K, McFadden S, et al. Increased platelet reactivity in patients with late-stage metastatic cancer. Cancer Med. 2013; 2: 564-70.

6. Sabrkhany S, Kuijpers MJE, van Kuijk SMJ, et al. A combination of platelet features allows detection of early-stage cancer. Eur J Cancer. 2017; 80: 5-13.

7. Best MG, Sol N, Kooi I, et al. RNA-seq of tumor-educated platelets enables blood-based pan-cancer, multiclass, and molecular pathway cancer diagnostics. Cancer Cell. 2015; 28: 666-76.

8. Menter DG, Tucker SC, Kopetz S, Sood AK, Crissman JD, Honn KV. Platelets and cancer: a casual or causal relationship: revisited. Cancer Metastasis Rev. 2014; 33: 231-69.

9. Lin RJ, Afshar-Kharghan V, Schafer Al. Paraneoplastic thrombocytosis: the secrets of tumor selfpromotion. Blood. 2014; 124: 184-7.

10. Goubran HA, Burnouf T, Radosevic M, El-Ekiaby M. The platelet-cancer loop. Eur J Intern Med. 2013; 24: 393-400.

11. Carmeliet P. Mechanisms of angiogenesis and arteriogenesis. Nat Med. 2000; 6: 389-95.

12. Klement GL, Yip TT, Cassiola F, et al. Platelets actively sequester angiogenesis regulators. Blood. 2009; 113: 2835-42.

13. Hanahan D, Weinberg RA. Hallmarks of cancer: the next generation. Cell. 2011; 144: 646-74.

14. Gross S, Rahal R, Stransky N, Lengauer C, Hoeflich KP. Targeting cancer with kinase inhibitors. J Clin Invest. 2015; 125: 1780-9.

15. Swieringa F, Kuijpers MJ, Heemskerk JW, van der Meijden PE. Targeting platelet receptor function in thrombus formation: the risk of bleeding. Blood Rev. 2014; 28: 9-21.

16. Levade M, Severin S, Gratacap MP, Ysebaert L, Payrastre B. Targeting kinases in cancer therapies: adverse effects on blood platelets. Curr Pharm Des. 2016; 22: 2315-22.

17. Wu P, Nielsen TE, Clausen MH. FDA-approved small-molecule kinase inhibitors. Trends Pharmacol Sci. 2015; 36: 422-39.

18. Fabian MA, Biggs WH, 3rd, Treiber DK, et al. A small molecule-kinase interaction map for clinical 
kinase inhibitors. Nat Biotechnol. 2005; 23: 329-36.

19. Karaman MW, Herrgard S, Treiber DK, et al. A quantitative analysis of kinase inhibitor selectivity. Nat Biotechnol. 2008; 26: 127-32.

20. Yu H, Steeghs N, Nijenhuis CM, Schellens JH, Beijnen JH, Huitema AD. Practical guidelines for therapeutic drug monitoring of anticancer tyrosine kinase inhibitors: focus on the pharmacokinetic targets. Clin Pharmacokinet. 2014; 53: 305-25.

21. Gotink KJ, Verheul HM. Antiangiogenic tyrosine kinase inhibitors: what is their mechanism of action? Angiogenesis. 2010; 13: 1-14.

22. Hartmann JT, Haap M, Kopp HG, Lipp HP. Tyrosine kinase inhibitors - a review on pharmacology, metabolism and side effects. Curr Drug Metab. 2009; 10: 470-81.

23. Eskens FA, Verweij J. The clinical toxicity profile of vascular endothelial growth factor (VEGF) and vascular endothelial growth factor receptor (VEGFR) targeting angiogenesis inhibitors; a review. Eur J Cancer. 2006; 42: 3127-39.

24. Moslehi JJ, Deininger M. Tyrosine kinase inhibitor-associated cardiovascular toxicity in chronic myeloid leukemia. J Clin Oncol. 2015; 33: 4210-8.

25. Bair SM, Choueiri TK, Moslehi J. Cardiovascular complications associated with novel angiogenesis inhibitors: emerging evidence and evolving perspectives. Trends in cardiovascular medicine. 2013; 23: 104-13.

26. Choueiri TK, Schutz FA, Je Y, Rosenberg JE, Bellmunt J. Risk of arterial thromboembolic events with sunitinib and sorafenib: a systematic review and meta-analysis of clinical trials. J Clin Oncol. 2010; 28: 2280-5.

27. Nieswandt B, Watson SP. Platelet-collagen interaction: is GPVI the central receptor? Blood. 2003; 102: 449-61.

28. Senis YA, Mazharian A, Mori J. Src family kinases: at the forefront of platelet activation. Blood. 2014; 124: 2013-24.

29. Watson SP, Auger JM, McCarty OJ, Pearce AC. GPVI and integrin alphallb beta3 signaling in platelets. J Thromb Haemost. 2005; 3: 1752-62.

30. Navarro-Núñez L, Langan SA, Nash GB, Watson SP. The physiological and pathophysiological roles of platelet CLEC-2. Thromb Haemost. 2013; 109: 991-8.

31. Shattil SJ, Newman PJ. Integrins: dynamic scaffolds for adhesion and signaling in platelets. Blood. 2004; 104: 1606-15.

32. Burkhart JM, Vaudel M, Gambaryan S, et al. The first comprehensive and quantitative analysis of human platelet protein composition allows the comparative analysis of structural and functional pathways. Blood. 2012; 120: e73-82.

33. Davis MI, Hunt JP, Herrgard S, et al. Comprehensive analysis of kinase inhibitor selectivity. Nat Biotechnol. 2011; 29: 1046-51.

34. Shatzel JJ, Olson SR, Tao DL, McCarty OJT, Danilov AV, DeLoughery TG. Ibrutinib-associated bleeding: pathogenesis, management and risk reduction strategies. J Thromb Haemost. 2017; 15: 835-47.

35. Molina-Cerrillo J, Alonso-Gordoa T, Gajate P, Grande E. Bruton's tyrosine kinase (BTK) as a promising target in solid tumors. Cancer Treat Rev. 2017; 58: 41-50.

36. Rauf F, Festa F, Park JG, et al. Ibrutinib inhibition of ERBB4 reduces cell growth in a WNT5A-dependent manner. Oncogene. 2018; 37: 2237-50.

37. Byrd JC, Furman RR, Coutre SE, et al. Targeting BTK with ibrutinib in relapsed chronic lymphocytic leukemia. N Engl J Med. 2013; 369: 32-42.

38. Caron F, Leong DP, Hillis C, Fraser G, Siegal D. Current understanding of bleeding with ibrutinib use: a systematic review and meta-analysis. Blood Adv. 2017; 1: 772-8.

39. Alberelli MA, Innocenti I, Sica S, Laurenti L, De Candia E. PO-54-Clinical and laboratory characterization of platelet dysfunction caused by ibrutinib treatment in patients with chronic lymphocytic leukemia. Thromb Res. 2016; 140 Suppl 1: S196.

40. Bye AP, Unsworth AJ, Vaiyapuri S, Stainer AR, Fry MJ, Gibbins JM. Ibrutinib inhibits platelet integrin alphallbbeta3 outside-in signaling and thrombus stability but not adhesion to collagen. Arterioscler Thromb Vasc Biol. 2015; 35: 2326-35.

41. Kamel S, Horton L, Ysebaert L, et al. Ibrutinib inhibits collagen-mediated but not ADP-mediated platelet aggregation. Leukemia. 2015; 29: 783-7. 


\section{Chapter 2}

42. Levade M, David E, Garcia C, et al. Ibrutinib treatment affects collagen and von Willebrand factordependent platelet functions. Blood. 2014; 124: 3991-5.

43. Lipsky AH, Farooqui MZ, Tian X, et al. Incidence and risk factors of bleeding-related adverse events in patients with chronic lymphocytic leukemia treated with ibrutinib. Haematologica. 2015; 100: 15718.

44. Rigg RA, Aslan JE, Healy LD, et al. Oral administration of Bruton's tyrosine kinase inhibitors impairs GPVI-mediated platelet function. Am J Physiol Cell Physiol. 2016; 310: C373-80.

45. Byrd JC, Harrington B, O'Brien S, et al. Acalabrutinib (ACP-196) in relapsed chronic lymphocytic leukemia. N Engl J Med. 2016; 374: 323-32.

46. Bye AP, Unsworth AJ, Desborough MJ, et al. Severe platelet dysfunction in NHL patients receiving ibrutinib is absent in patients receiving acalabrutinib. Blood Adv. 2017; 1: 2610-23.

47. Yang K, Fu LW. Mechanisms of resistance to BCR-ABL TKIs and the therapeutic strategies: A review. Crit Rev Oncol Hematol. 2015; 93: 277-92.

48. Breccia M, Stefanizzi C, Cannella L, et al. Differences in hematological and non-hematological toxicity during treatment with imatinib in patients with early and late chronic phase chronic myeloid leukemia. Leuk Lymphoma. 2008; 49: 2328-32.

49. Kostos L, Burbury K, Srivastava G, Prince HM. Gastrointestinal bleeding in a chronic myeloid leukaemia patient precipitated by dasatinib-induced platelet dysfunction: Case report. Platelets. 2015; 26: 80911.

50. Mustafa Ali MK, Sabha MM, Al-Rabi KH. Spontaneous subdural hematoma in a patient with Philadelphia chromosome-positive acute lymphoblastic leukemia with normal platelet count after dasatinib treatment. Platelets. 2015; 26: 491-4.

51. Gratacap MP, Martin V, Valéra MC, et al. The new tyrosine-kinase inhibitor and anticancer drug dasatinib reversibly affects platelet activation in vitro and in vivo. Blood. 2009; 114: 1884-92.

52. Quintás-Cardama A, Han X, Kantarjian H, Cortes J. Tyrosine kinase inhibitor-induced platelet dysfunction in patients with chronic myeloid leukemia. Blood. 2009; 114: 261-3.

53. Li R, Grosser T, Diamond SL. Microfluidic whole blood testing of platelet response to pharmacological agents. Platelets. 2017; 28: 457-62.

54. Shah NP, Kantarjian HM, Kim DW, et al. Intermittent target inhibition with dasatinib 100 mg once daily preserves efficacy and improves tolerability in imatinib-resistant and-intolerant chronic-phase chronic myeloid leukemia. J Clin Oncol. 2008; 26: 3204-12.

55. Mazharian A, Ghevaert C, Zhang L, Massberg S, Watson SP. Dasatinib enhances megakaryocyte differentiation but inhibits platelet formation. Blood. 2011.

56. Alhawiti N, Burbury KL, Kwa FA, et al. The tyrosine kinase inhibitor, nilotinib potentiates a prothrombotic state. Thromb Res. 2016; 145: 54-64.

57. Loren CP, Aslan JE, Rigg RA, et al. The BCR-ABL inhibitor ponatinib inhibits platelet immunoreceptor tyrosine-based activation motif (ITAM) signaling, platelet activation and aggregate formation under shear. Thromb Res. 2015; 135: 155-60.

58. Neelakantan P, Marin D, Laffan M, Goldman J, Apperley J, Milojkovic D. Platelet dysfunction associated with ponatinib, a new pan BCR-ABL inhibitor with efficacy for chronic myeloid leukemia resistant to multiple tyrosine kinase inhibitor therapy. Haematologica. 2012; 97: 1444-.

59. Pasvolsky O, Leader A, lakobishvili Z, Wasserstrum Y, Kornowski R, Raanani P. Tyrosine kinase inhibitor associated vascular toxicity in chronic myeloid leukemia. Cardiooncology. 2015; 1: 5.

60. Je Y, Schutz FAB, Choueiri TK. Risk of bleeding with vascular endothelial growth factor receptor tyrosine-kinase inhibitors sunitinib and sorafenib: a systematic review and meta-analysis of clinical trials. Lancet Oncol. 2009; 10: 967-74.

61. Sabrkhany S, Griffioen AW, Pineda S, et al. Sunitinib uptake inhibits platelet function in cancer patients. Eur J Cancer. 2016; 66: 47-54.

62. Hamilton Z, Lee HJ, Jimenez J, et al. Change in platelet count as a prognostic indicator for response to primary tyrosine kinase inhibitor therapy in metastatic renal cell carcinoma. BJU Int. 2016; 118: 927-34.

63. Crist M, Hansen E, Chablani L, Guancial E. Examining the bleeding incidences associated with targeted therapies used in metastatic renal cell carcinoma. Crit Rev Oncol Hematol. 2017; 120: 151-62.

64. Keating GM. Afatinib: a review of its use in the treatment of advanced non-small cell lung cancer. 
Drugs. 2014; 74: 207-21.

65. Cao H, Bhuyan AAM, Umbach AT, Bissinger R, Gawaz M, Lang F. Inhibitory effect of afatinib on platelet activation and apoptosis. Cell Physiol Biochem. 2017; 43: 2264-76.

66. Cardoso HJ, Figueira MI, Socorro S. The stem cell factor (SCF)/c-KIT signalling in testis and prostate cancer. J Cell Commun Signal. 2017; 11: 297-307.

67. Hitchcock IS, Kaushansky K. Thrombopoietin from beginning to end. Br J Haematol. 2014; 165: 25968.

68. Quintas-Cardama A, Verstovsek S. Spleen deflation and beyond: the pros and cons of Janus kinase 2 inhibitor therapy for patients with myeloproliferative neoplasms. Cancer. 2012; 118: 870-7.

69. Moore SF, Hunter RW, Harper MT, et al. Dysfunction of the PI3 kinase/Rap1/integrin alpha(IIb)beta(3) pathway underlies ex vivo platelet hypoactivity in essential thrombocythemia. Blood. 2013; 121: 1209-19.

70. Lu WJ, Lin KC, Huang SY, et al. Role of a Janus kinase 2-dependent signaling pathway in platelet activation. Thromb Res. 2014; 133: 1088-96.

71. Andrews RK, Arthur JF, Gardiner EE. Targeting GPVI as a novel antithrombotic strategy. J Blood Med. 2014; 5: 59-68.

72. Etulain J, Fondevila C, Negrotto S, Schattner M. Platelet-mediated angiogenesis is independent of VEGF and fully inhibited by aspirin. Br J Pharmacol. 2013; 170: 255-65.

73. Mezouar S, Darbousset R, Dignat-George F, Panicot-Dubois L, Dubois C. Inhibition of platelet activation prevents the P-selectin and integrin-dependent accumulation of cancer cell microparticles and reduces tumor growth and metastasis in vivo. Int J Cancer. 2015; 136: 462-75.

74. Rothwell PM, Wilson M, Price JF, Belch JF, Meade TW, Mehta Z. Effect of daily aspirin on risk of cancer metastasis: a study of incident cancers during randomised controlled trials. Lancet. 2012; 379: 1591601.

75. Mitrugno A, Sylman JL, Ngo AT, et al. Aspirin therapy reduces the ability of platelets to promote colon and pancreatic cancer cell proliferation: Implications for the oncoprotein c-MYC. Am J Physiol Cell Physiol. 2017; 312: C176-C89.

76. Verheul HM, Hoekman K, Luykx-de Bakker S, et al. Platelet: transporter of vascular endothelial growth factor. Clin Cancer Res. 1997; 3: 2187-90.

77. Peterson JE, Zurakowski D, Italiano JE, Jr., et al. VEGF, PF4 and PDGF are elevated in platelets of colorectal cancer patients. Angiogenesis. 2012; 15: 265-73.

78. Verheul HM, Lolkema MP, Qian DZ, et al. Platelets take up the monoclonal antibody bevacizumab. Clin Cancer Res. 2007; 13: 5341-7.

79. Williams M, Liu ZW, Woolf D, et al. Change in platelet levels during radiotherapy with concurrent and adjuvant temozolomide for the treatment of glioblastoma: a novel prognostic factor for survival. J Cancer Res Clin Oncol. 2012; 138: 1683-8.

80. Chen F, Maridakis V, O'Neill E A, et al. A randomized clinical trial comparing point-of-care platelet function assays and bleeding time in healthy subjects treated with aspirin or clopidogrel. Platelets. 2012; 23: 249-58.

81. Nagy M, Mastenbroek TG, Mattheij NJA, et al. Variable impairment of platelet functions in patients with severe, genetically linked immune deficiencies. Haematologica. 2018; 103: 540-9.

82. Carrier M, Le Gal G, Wells PS, Fergusson D, Ramsay T, Rodger MA. Systematic review: the Trousseau syndrome revisited: should we screen extensively for cancer in patients with venous thromboembolism? Ann Intern Med. 2008; 149: 323-33.

83. Li J, Zhao X, Chen L, et al. Safety and pharmacokinetics of novel selective vascular endothelial growth factor receptor-2 inhibitor YN968D1 in patients with advanced malignancies. BMC Cancer. 2010; 10: 529.

84. Keller G, Schafhausen P, Brummendorf TH. Bosutinib: a dual SRC/ABL kinase inhibitor for the treatment of chronic myeloid leukemia. Expert Rev Hematol. 2009; 2: 489-97.

85. Johnson PJ, Qin S, Park JW, et al. Brivanib versus sorafenib as first-line therapy in patients with unresectable, advanced hepatocellular carcinoma: results from the randomized phase III BRISK-FL study. J Clin Oncol. 2013; 31: 3517-24.

86. Choueiri TK, Escudier B, Powles T, et al. Cabozantinib versus everolimus in advanced renal-cell carcinoma. N Engl J Med. 2015; 373: 1814-23. 


\section{Chapter 2}

87. Langenberg $\mathrm{MH}$, van Herpen $\mathrm{CM}$, De Bono J, et al. Effective strategies for management of hypertension after vascular endothelial growth factor signaling inhibition therapy: results from a phase II randomized, factorial, double-blind study of Cediranib in patients with advanced solid tumors. J Clin Oncol. 2009; 27: 6152-9.

88. Soria J-C, Tan DSW, Chiari R, et al. First-line ceritinib versus platinum-based chemotherapy in advanced ALK-rearranged non-small-cell lung cancer (ASCEND-4): a randomised, open-label, phase 3 study. Lancet. 2017; 389: 917-29.

89. Boespflug A, Thomas L. Cobimetinib and vemurafenib for the treatment of melanoma. Expert Opin Pharmacother. 2016; 17: 1005-11.

90. Shaw AT, Yasothan U, Kirkpatrick P. Crizotinib. Nat Rev Drug Discov. 2011; 10: 897-8.

91. Gibney GT, Zager JS. Clinical development of dabrafenib in BRAF mutant melanoma and other malignancies. Expert Opin Drug Metab Toxicol. 2013; 9: 893-9.

92. Reckamp KL, Giaccone G, Camidge DR, et al. A phase 2 trial of dacomitinib (PF-00299804), an oral, irreversible pan-HER (human epidermal growth factor receptor) inhibitor, in patients with advanced non-small cell lung cancer after failure of prior chemotherapy and erlotinib. Cancer. 2014; 120: 114554.

93. Aguilera DG, Tsimberidou AM. Dasatinib in chronic myeloid leukemia: a review. Ther Clin Risk Manag. 2009; 5: 281-9.

94. Joensuu H, Blay JY, Comandone $\mathrm{A}$, et al. Dovitinib in patients with gastrointestinal stromal tumour refractory and/or intolerant to imatinib. Br J Cancer. 2017.

95. Sharman J, Hawkins M, Kolibaba K, et al. An open-label phase 2 trial of entospletinib (GS-9973), a selective spleen tyrosine kinase inhibitor, in chronic lymphocytic leukemia. Blood. 2015; 125: 233643.

96. Rocha-Lima CM, Raez LE. Erlotinib (Tarceva) for the treatment of non-small-cell lung cancer and pancreatic cancer. P T. 2009; 34: 554-64.

97. McAdoo SP, Tam FWK. Fostamatinib Disodium. Drugs Future. 2011; 36: 273.

98. Shi Y, Zhang L, Liu X, et al. Icotinib versus gefitinib in previously treated advanced non-small-cell lung cancer (ICOGEN): a randomised, double-blind phase 3 non-inferiority trial. Lancet Oncol. 2013; 14: 953-61.

99. Blay J-Y, von Mehren M. Nilotinib: a novel, selective tyrosine kinase inhibitor. Sem Oncol. 2011; 38: S3-S9.

100. Kim S-B, Dent R, Im S-A, et al. Ipatasertib plus paclitaxel versus placebo plus paclitaxel as first-line therapy for metastatic triple-negative breast cancer (LOTUS): a multicentre, randomised, doubleblind, placebo-controlled, phase 2 trial. Lancet Oncol. 2017; 18: 1360-72.

101. Blackwell KL, Burstein HJ, Storniolo AM, et al. Randomized study of lapatinib alone or in combination with trastuzumab in women with ErbB2-positive, trastuzumab-refractory metastatic breast cancer. J Clin Oncol. 2010; 28: 1124-30.

102. Schlumberger $M$, Tahara $M$, Wirth $L$, et al. Lenvatinib versus placebo in radioiodine-refractory thyroid cancer. N Engl J Med. 2015; 372: 621-30.

103. Richeldi L, du Bois RM, Raghu G, et al. Efficacy and safety of nintedanib in idiopathic pulmonary fibrosis. N Engl J Med. 2014; 370: 2071-82.

104. Goss G, Tsai C-M, Shepherd FA, et al. Osimertinib for pretreated EGFR Thr790Met-positive advanced non-small-cell lung cancer (AURA2): a multicentre, open-label, single-arm, phase 2 study. Lancet Oncol. 2016; 17: 1643-52.

105. Jain $\mathrm{P}$, Kantarjian $\mathrm{H}$, Jabbour $\mathrm{E}$, et al. Ponatinib as first-line treatment for patients with chronic myeloid leukaemia in chronic phase: a phase 2 study. Lancet Haematol. 2015; 2: e376-e83.

106. Ferraro D, Zalcberg J. Regorafenib in gastrointestinal stromal tumors: clinical evidence and place in therapy. Ther Adv Med Oncol. 2014; 6: 222-8.

107. Casaluce F, Sgambato A, Maione P, Sacco PC, Santabarbara G, Gridelli C. Selumetinib for the treatment of non-small cell lung cancer. Exp Opin Invest Drugs. 2017; 26: 973-84.

108. Lugowska I, Koseła-Paterczyk H, Kozak K, Rutkowski P. Trametinib: a MEK inhibitor for management of metastatic melanoma. Onco Targets Ther. 2015; 8: 2251-9.

109. Wells SA, Robinson BG, Gagel RF, et al. Vandetanib in patients with locally advanced or metastatic medullary thyroid cancer: a randomized, double-blind phase III trial. J Clin Oncol. 2012; 30: 134-41. 
110. Hecht JR, Trarbach T, Hainsworth JD, et al. Randomized, placebo-controlled, phase III study of firstline oxaliplatin-based chemotherapy plus PTK787/ZK 222584, an oral vascular endothelial growth factor receptor inhibitor, in patients with metastatic colorectal adenocarcinoma. J Clin Oncol. 2011; 29: 1997-2003.

111. Bollag G, Tsai J, Zhang J, et al. Vemurafenib: the first drug approved for BRAF-mutant cancer. Nat Rev Drug Discov. 2012; 11: 873. 

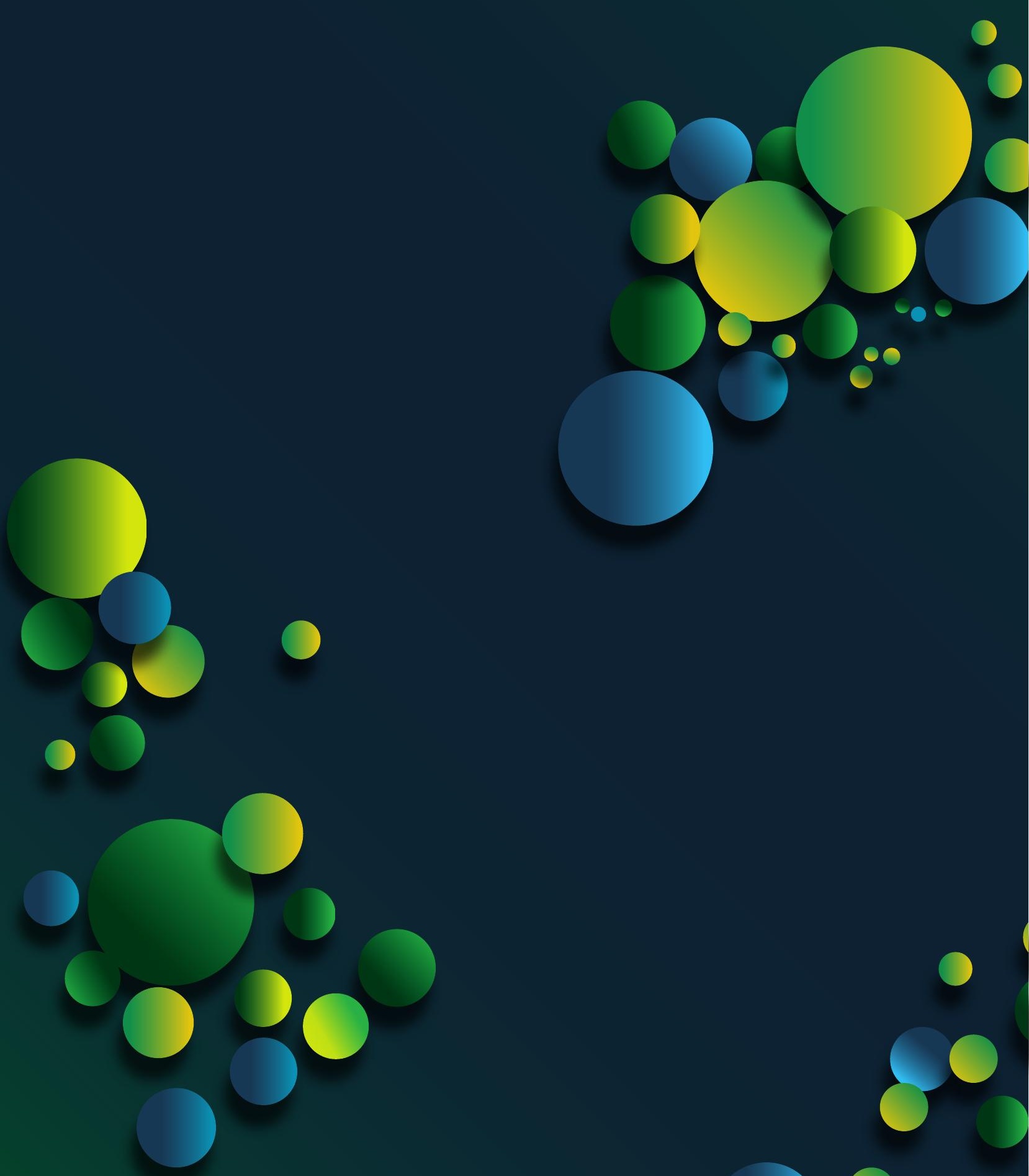


\section{Chapter 3}

Tyrosine kinase inhibitor pazopanib inhibits platelet procoagulant activity in renal cell carcinoma patients

Tullemans BME, Nagy M, Sabrkhany S, Griffioen AW, oude Egbrink MGA, Aarts MJB, Heemskerk JWM and Kuijpers MJE

Front Cardiovasc Med 2018; 5:142 Reprinted with permission 


\section{Chapter 3}

\section{Abstract}

Pazopanib is an angiostatic tyrosine kinase inhibitor (TKI) presently used for cancer treatment, particularly in patients with renal cell carcinoma (RCC). This treatment can be accompanied by mild bleeding as an adverse effect. Given the role of protein tyrosine kinases in platelet activation processes, we investigated whether and how pazopanib can affect platelet functions in purified systems and during treatment of advanced RCC patients. In isolated platelets from healthy volunteers, pazopanib dose-dependently reduced collagen-induced integrin activation and secretion, as well as platelet aggregation. Pazopanib addition diminished glycoprotein (GP)VI-dependent tyrosine phosphorylation of multiple platelet proteins, including the tyrosine kinase Syk. Furthermore, pazopanib inhibited GPVI-induced $\mathrm{Ca}^{2+}$ elevation, resulting in reduced exposure of the procoagulant phospholipid phosphatidylserine (PS). Upon perfusion of control blood over a collagen surface, pazopanib inhibited thrombus size as well as PS exposure. Blood samples from ten RCC patients were also analysed before and after 14 days of pazopanib treatment as monotherapy. This treatment caused an overall lowering in platelet count, with three out of ten patients experiencing mild bleeding. Platelets isolated from pazopanib-treated patients showed a significant lowering of PS exposure upon activation. In addition, platelet procoagulant activity was inhibited in thrombi formed under flow conditions. Control experiments indicated that higher pazopanib concentrations were required to inhibit GPVI-mediated PS exposure in the presence of plasma. Together, these results indicated that pazopanib suppresses GPVI-induced platelet activation responses in a way partly antagonized by the presence of plasma. In treated cancer patients, pazopanib effects were confined to a reduction in GPVI-dependent PS exposure. Together with the reduced platelet count, this may explain the mild bleeding tendency observed in pazopanib-treated patients. 


\section{Introduction}

Tyrosine kinase inhibitors (TKIs) are widely approved drugs, aiming to target tyrosine kinase signalling pathways that regulate uncontrolled cellular growth and proliferation. Currently, several TKIs are in clinical use for the treatment of malignancies, such as lung, breast, kidney, and neuroendocrine pancreatic cancers as well as gastro-intestinal stromal tumours and chronic myeloid leukaemia ${ }^{1-3}$. Their common way of action is by competition with adenosine triphosphate (ATP) in the conserved catalytic binding site in the protein tyrosine kinase superfamily. In spite of this action mechanism, individual TKIs can target partially different spectra of intracellular tyrosine kinases, can have different pharmacokinetics, and vary in their adverse effects ${ }^{1}$. Commonly, TKIs are clinically applied as a multi-target therapy to intervene in tumour proliferation ${ }^{4,5}$. Specific targets are the receptors for vascular endothelial growth factor (VEGF), platelet-derived growth factor (PDGF) and epidermal growth factor (EGF), which are all involved in (tumour) angiogenesis ${ }^{3,}$ 6 . The expected effects are to reduce tumour lesions, delay disease development, and thus prolong the progression-free survival of patients ${ }^{7}$.

Platelets contain several protein tyrosine kinases as key signal transducers, which regulate the function of platelets in haemostasis ${ }^{8}$. Downstream of glycoprotein (GP)VI (collagen receptor), GPIb-IX-V (von Willebrand receptor) and CLEC-2 (podoplanin receptor), Src family tyrosine kinases control the signalling routes to most platelet responses ${ }^{8-10}$. Activation of GPVI also implies tyrosine phosphorylation of the immunoreceptor tyrosine-based activation motif (ITAM) present on the Fc-receptor $y$-chain, which is co-expressed with GPVI ${ }^{11}$. This results in activation of the protein tyrosine kinase $\mathrm{Syk}^{10,12}$, and further downstream Bruton tyrosine kinase (Btk), culminating in the phosphorylation and activation of phospholipase $C_{\gamma 2}$ (PLC 2 2), an event required for integrin activation and granule secretion. A similar set of protein tyrosine kinases (Src-family kinases, Syk and JAK isoforms) is known to play a critical role in megakaryocyte development and thrombocytopoiesis ${ }^{13}$.

Considering the role of protein tyrosine kinases in both platelet activation and platelet formation, it can be expected that treatment of patients with a broad-spectrum TKI affects haemostasis. Indeed, for several TKIs, anti-haemostatic effects have been reported, like anaemia, neutropenia, thrombocytopenia, and bleeding incidences ${ }^{14-19}$. In particular, the TKIs sunitinib, ibrutinib, and ponatinib may cause a bleeding risk that relates to impaired GPVI-induced signalling, platelet aggregation, and thrombus formation ${ }^{14,15,18}$. Also for other TKIs a decrease in platelet count has been described upon treatment ${ }^{18,20-22}$.

Pazopanib is currently used as a first-line therapy for advanced clear-cell renal cell carcinoma (RCC), the most common type of kidney cancer in adults. Pazopanib is aimed to target the VEGF and PDGF receptors, stem-cell factor receptor cKit and Flt- $3^{23}$. The inhibition of these receptors decreases tumour angiogenesis and growth, and hence prolongs patient survival ${ }^{24,25}$. In vitro studies have indicated that pazopanib also inhibits several other tyrosine kinase-linked 
receptors, including fibroblast growth factor receptor, IL-2 receptor inducible T-cell kinase (Itk), leukocyte-specific protein tyrosine kinase (LCk), and the glycoprotein receptor C-Fms ${ }^{24}$. In addition, other in vitro kinase targets of pazopanib have been described, of which ABL1, ABL2, FGR, Src, Fyn and Lck are present in platelets ${ }^{26,27}$. Patients are commonly treated with a high daily dose of pazopanib (800 mg), resulting in a steady-state plasma concentration of up to $45 \mu \mathrm{g} / \mathrm{mL}$ after several weeks ${ }^{24}$. This treatment regimen can reduce the platelet count and lead to bleeding events $^{24}$. Since effects of pazopanib on platelet function have not been reported, we aimed to investigate this in vitro and ex vivo, using blood from RCC patients and control subjects.

\section{Materials and Methods}

Materials

Pazopanib (Votrient) was obtained from LC Laboratories (Woburn MA, USA). Arachidonic acid was obtained from Bio/DATA Corporation (Horsham PA, USA), fibrillar Horm type I collagen from Takeda (Hoofddorp, The Netherlands), thrombin from Enzyme Research Laboratories (South Bend IN, USA), thrombin receptor activating peptide 6 (TRAP-6) from Bachem (Bubendorf, Switzerland) and U46619 (thromboxane $\mathrm{A}_{2}$ receptor agonist) from Cayman Chemicals (Ann Arbor MI, USA). Fluorescein isothiocyanate (FITC)-labelled PAC-1 monoclonal antibody (mAb) against activated human integrin $\alpha_{11 b} \beta_{3}$ was from BD Bioscience (Franklin Lakes NJ, USA; nr. 340507), while FITC-labelled anti-human CD62 mAb was from Beckman Coulter (Sydney, Australia; nr. 65050). Collagen-related peptide (CRP-XL) was obtained from the University of Cambridge (Cambridge, UK). 2Methylthio-adenosine-diphosphate (2Me-S-ADP), D-phenylalanyl-prolylarginyl chloromethyl ketone (PPACK) and mouse anti-Syk antibody were purchased from Santa Cruz Biotechnology (Dallas TX, USA). Fibrinogen and unfractionated heparin were obtained from Sigma-Aldrich (Saint Louis MO, USA). FITC-labelled annexin A5 was from Pharmatarget (Maastricht, The Netherlands). Fura-2-AM was from Invitrogen (Carlsbad CA, USA); Pluronic F-127 from Molecular Probes (Eugene OR, USA). For Western blotting, the following antibodies were used: mouse anti-phosphotyrosine mAb (clone 4G10) obtained from Millipore (Billerica MA, USA), rabbit anti-tubulin Ab from Abcam (Cambridge, UK), rabbit anti-phospho Syk Tyr525/526, HRPconjugated anti-rabbit-IgG from Cell Signalling, (Leiden, The Netherlands) and HRP-conjugated anti-mouse-IgG from VWR international (Amsterdam, The Netherlands).

\section{Blood collection from patients and healthy volunteers}

The study was approved by the medical ethics committee of the Maastricht University Medical Centre+ (MUMC+, The Netherlands). All participants provided written informed consent in accordance with the Declaration of Helsinki. Blood was obtained from healthy volunteers and ten patients diagnosed with metastatic renal cell carcinoma at the Department of Medical Oncology of MUMC+. Patients were included, if eligible for treatment with pazopanib as a single 
agent (800 mg/day). Excluded were subjects who used anticoagulants or platelet inhibitory drugs. From healthy volunteers, one blood sample was collected, while the patients donated two blood samples: one day before and at 14 days after starting pazopanib treatment, i.e., when a steadystate plasma concentration was reached ${ }^{24}$. Blood samples were collected from the antecubital vein into $3.2 \%$ trisodium citrate; the first $5 \mathrm{~mL}$ of blood was discarded after which $10 \mathrm{~mL}$ was collected.

\section{Blood composition and platelet isolation}

Haematological parameters, including platelet count, were determined with a Sysmex XP300 (Chuo-ku Kobe, Japan). Washed platelets were obtained as described ${ }^{28}$. In brief, plateletrich plasma (PRP) was collected after 15 minutes centrifugation at $240 \mathrm{~g}$, followed by a washing step. Washed platelets were resuspended into Hepes buffer pH 7.45 [10 mM Hepes, 136 mM $\mathrm{NaCl}, 2.7 \mathrm{mM} \mathrm{KCl}, 2 \mathrm{mM} \mathrm{MgCl}, 0.1 \%$ glucose and $0.1 \%$ bovine serum albumin (BSA)]. Platelet count was adjusted, as required for the particular assay.

Blood from healthy volunteers was used for in vitro experiments of pazopanib effects. Samples of whole blood, PRP or washed platelets were pre-incubated with pazopanib or vehicle (dimethylsulfoxide) for 10 minutes at $37^{\circ} \mathrm{C}$.

\section{Light transmission aggregometry}

Aggregation of platelets, washed or in PRP $\left(250 \times 10^{9}\right.$ platelets/L) was measured using a Chronolog aggregometer (Havertown PA, USA) under constant stirring $\left(37^{\circ} \mathrm{C}\right)$; pazopanib $(5,10$ or $30 \mu \mathrm{M})$ or vehicle was present as indicated. Aggregation responses were quantified as maximal amplitude in light transmission ${ }^{29}$. Aggregation of washed platelets was induced with collagen (1 $\mu \mathrm{g} / \mathrm{mL}), 2 \mathrm{Me}-\mathrm{S}-A D P(1 \mu \mathrm{M})$ in the presence of fibrinogen $(25 \mu \mathrm{g} / \mathrm{mL})$, thrombin $(1 \mathrm{nM})$, TRAP$6(10 \mu \mathrm{M}), \mathrm{U} 46619(1 \mu \mathrm{M})$, or arachidonic acid $(10 \mu \mathrm{M})$. Aggregation of platelets in PRP was induced with collagen $(1 \mu \mathrm{g} / \mathrm{mL})$.

\section{Flow cytometry}

Washed platelets $\left(100 \times 10^{9} / \mathrm{L}\right)$ were incubated for 10 minutes at $37^{\circ} \mathrm{C}$ with vehicle or pazopanib $(10 \mu \mathrm{M})$. The cells in Hepes buffer $\mathrm{pH} 7.45$ containing $2 \mathrm{mM} \mathrm{CaCl}_{2}$ were then stimulated with CRP-XL $(1 \mu \mathrm{g} / \mathrm{mL}), 2 \mathrm{Me}-\mathrm{S}-\mathrm{ADP}(1 \mu \mathrm{M})$, or thrombin $(1 \mathrm{nM})$. Using described flow cytometry procedures, integrin $\alpha_{11 \mathrm{~b}} \beta_{3}$ activation and P-selectin expression were determined with FITCconjugated PAC1 mAb (1:10) and FITC-conjugated anti-CD62P mAb (1:10), respectively ${ }^{30}$. For the measurement of PS exposure, platelets were stimulated with CRP-XL $(5 \mu \mathrm{g} / \mathrm{mL})$ and thrombin (4 $\mathrm{nM})$ for 60 minutes at $37^{\circ} \mathrm{C}^{31}$. Exposure of PS was determined with FITC-conjugated annexin A5 $(1 \mu \mathrm{g} / \mathrm{mL})$. 


\section{Chapter 3}

To assess PS exposure in the presence of plasma, platelet count of washed platelets or PRP were adjusted to $100 \times 10^{9}$ platelets/L. Washed platelet were diluted in Hepes buffer $\mathrm{pH} 7.45$ with $2 \mathrm{mM} \mathrm{CaCl}_{2}$; PRP was diluted with autologous plasma supplemented and $6.3 \mathrm{mM} \mathrm{CaCl}{ }_{2}$ plus $3.2 \mathrm{mM} \mathrm{MgCl}_{2}$. Washed platelets and PRP were mixed in various ratios to obtain 0, 10, 30, 50 and $100 \%$ plasma. The mixed samples were preincubated with pazopanib $(1,5,10,30,50,75$ or 100 $\mu \mathrm{M})$ or vehicle for 10 minutes at $37^{\circ} \mathrm{C}$, and then activated with CRP-XL $(5 \mu \mathrm{g} / \mathrm{mL})$ and TRAP-6 (15 $\mu \mathrm{M})$ in the presence of PPACK $(40 \mu \mathrm{M})$. Exposure of PS was determined after 60 minutes at $37^{\circ} \mathrm{C}$ with FITC-conjugated annexin A5 $(1 \mu \mathrm{g} / \mathrm{mL})$. Flow cytometric measurements were performed in duplicate using a BD Accuri C6TM flow cytometer and corresponding software (Erembodegem, Belgium).

\section{Whole blood perfusion experiments}

Whole blood perfusion experiments were performed as described before ${ }^{32}$. In short, citrateanticoagulated blood samples were incubated with pazopanib $(30 \mu \mathrm{M})$ or vehicle for 10 minutes at room temperature. After recalcification in the presence of thrombin inhibitor (40 $\mu \mathrm{M}$ PPACK, $6.3 \mathrm{mM} \mathrm{CaCl}_{2}, 3.2 \mathrm{mM} \mathrm{MgCl}_{2}$, f.c.), the samples were perfused through a transparent parallelplate flow chamber, containing a coverslip coated with type I collagen (50 $\mu \mathrm{g} / \mathrm{mL})$ at a wall-shear rate of $1000 \mathrm{~s}^{-1}$. After 4 minutes, thrombi formed on coverslip were stained with FITC-conjugated annexin A5 $\left(1 \mu \mathrm{g} / \mathrm{mL}\right.$ in Hepes buffer $\mathrm{pH} 7.45$, containing $2 \mathrm{mM} \mathrm{CaCl}_{2}$ and $1 \mathrm{U} / \mathrm{mL}$ heparin). At least 10 random brightfield and fluorescence images were captured with an EVOS microscope (Bothell WA, USA). Microscopic digital images were analysed for platelet deposition (\% of surface area coverage, SAC), multilayer \% SAC, integrated feature size and staining for PS (\% SAC), using ImageJ 1.45s software (ImageJ ecosystem, from imagej.nih.gov/ij/). The integrated feature size is a parameter of platelet aggregation, taking into account the proportional contribution of large and small thrombi on microspots ${ }^{33}$. Further details of image analysis are described elsewhere ${ }^{34}$.

\section{Cytosolic $\mathrm{Ca}^{2+}$ measurements}

Washed platelets were incubated with Fura-2-AM (3 $\mu \mathrm{M})$ in the presence of pluronic (600 $\mu \mathrm{M})$ for 45 minutes at $37^{\circ} \mathrm{C}$. After another wash step, the Fura-2 loaded platelets $\left(100 \times 10^{9} / \mathrm{L}\right)$ were used for cytosolic $\mathrm{Ca}^{2+}\left(\left[\mathrm{Ca}^{2+}\right]_{\mathrm{i}}\right)$ measurements, as described previously ${ }^{35}$. In brief, using polystyrene cuvettes, platelets in suspension $(0.5 \mathrm{~mL})$ were pre-incubated with pazopanib $(10 \mu \mathrm{M})$ or vehicle for 8 minutes at room temperature and 2 minutes at $37^{\circ} \mathrm{C}$. After baseline measurement, $\mathrm{CaCl}_{2}(1$ $\mathrm{mM}$ ) was added, followed by an agonist. Changes in Fura-2 fluorescence were measured by ratio fluorometry at dual excitation wavelengths of 340 and $380 \mathrm{~nm}$ and an emission wavelength of $510 \mathrm{~nm}$. After correction for background fluorescence, ratio values were converted into levels of $\left[\mathrm{Ca}^{2+}\right]_{i}$. Maximal rises in $\left[\mathrm{Ca}^{2+}\right]_{i}$ and $\left[\mathrm{Ca}^{2+}\right]_{i}$-time integrals (4 minutes) were determined ${ }^{36}$. 


\section{Western blotting}

Washed platelets $\left(500 \times 10^{9} / \mathrm{L}\right)$ were pre-incubated with pazopanib $(10$ or $30 \mu \mathrm{M})$ or vehicle and stimulated under stirring conditions with CRP-XL $(5 \mu \mathrm{g} / \mathrm{mL})$ or left unstimulated. Samples were lysed with NP40 lysis buffer, supplemented with a cocktail of phosphatase inhibitors, and protein content was determined using a BioRad protein assay (Hercules CA, USA). Platelet lysates were separated by polyacrylamide gel electrophoresis and subjected to standard western blotting. Blots were stained for tyrosine phosphorylation profile as described ${ }^{18}$, using anti-phosphotyrosine mAb 4G10 (1:2000) and secondary HRP-conjugated Ab (1:500). Specific phosphorylation of Syk was visualized, as described ${ }^{37}$, using anti-Syk Tyr525/526 mAb (1:1000) and secondary HRPconjugated secondary Ab (1:500). Total Syk was determined by reprobing with anti-Syk mAb (1:1000) and HRP-conjugated secondary Ab (1:1000). As a control for total platelet proteins, blots were also probed for $\alpha$-tubulin (1:1000). The intensity of stained bands was analysed with ImageJ 1.45 s software.

\section{Statistical analysis}

Data were checked for Gaussian distribution using the Kolmogorov-Smirnov Normality test. Normally distributed data are presented as means \pm SEM; data that are not normally distributed are presented as median \pm interquartile ranges. Statistical significance between in vitro datasets (vehicle vs. pazopanib-treated samples) was determined using paired t-test. Paired datasets of the patients (before and after pazopanib therapy) were compared using the Wilcoxon matchedpairs signed-rank test. When more than 2 conditions were compared, a one-way ANOVA was used. GraphPad Prism 5.0 software (La Jolla CA, USA) was used for statistical analyses. A P-value less than 0.05 was considered to be statistically significant.

\section{Results}

\section{Pazopanib in vitro suppresses collagen-and ADP-induced platelet aggregation, secretion and PS exposure}

Platelet activation in haemostasis involves multiple agonists and their receptors ${ }^{38}$. Adhesion of platelets to extracellular matrix proteins, like collagen, is followed by platelet activation (characterized by integrin $\alpha_{\| b} \beta_{3}$ activation and secretion), aggregation (via released ADP and thromboxane $A_{2}$ ) and thrombus formation. Highly activated platelets also expose the procoagulant phospholipid PS, which promotes thrombin generation and fibrin formation ${ }^{39,40}$. We first investigated the effect of pazopanib on the aggregation response of washed platelets to collagen (acting via GPVI) or ADP (acting via P2Y receptors). Dose-response curves indicated near-complete inhibition with either agonist already at a relatively low dose of $10 \mu \mathrm{M}$ pazopanib (Figure 3.1A). In following experiments, the concentration of $10 \mu \mathrm{M}$ appeared to suppress platelet aggregation with ADP, arachidonic acid and $U 46619$ (thromboxane $A_{2}$ analogue). On the other 
hand, platelet aggregation induced by thrombin or the PAR1 receptor agonist, TRAP-6, was not affected by pazopanib at this dose (Figure 3.1B).

Subsequently, flow cytometry was used to determine the effects of pazopanib on specific platelet responses, i.e. integrin $\alpha_{\| 1} \beta_{3}$ activation and $\alpha$-granule secretion (P-selectin expression). Markedly, pazopanib at $10 \mu \mathrm{M}$ strongly inhibited integrin activation and granule secretion induced by the GPVI receptor agonist (CRP-XL), with no or limited effect in response to ADP or thrombin stimulation (Figure 3.1C). Pazopanib also reduced CRP-XL plus thrombin-induced PS exposure by $>50 \%$ (Figure 3.1D). Taken together, these results indicate that, in washed platelets, pazopanib is

$\mathrm{Ai})$

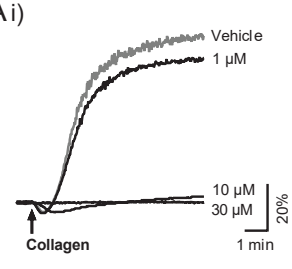

ii)

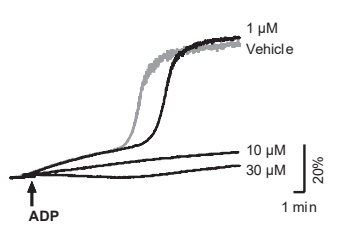

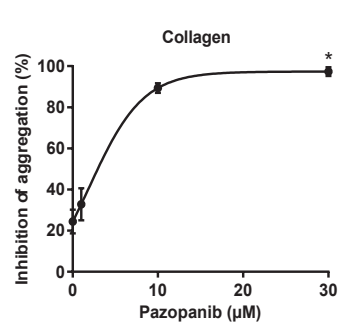

ADP
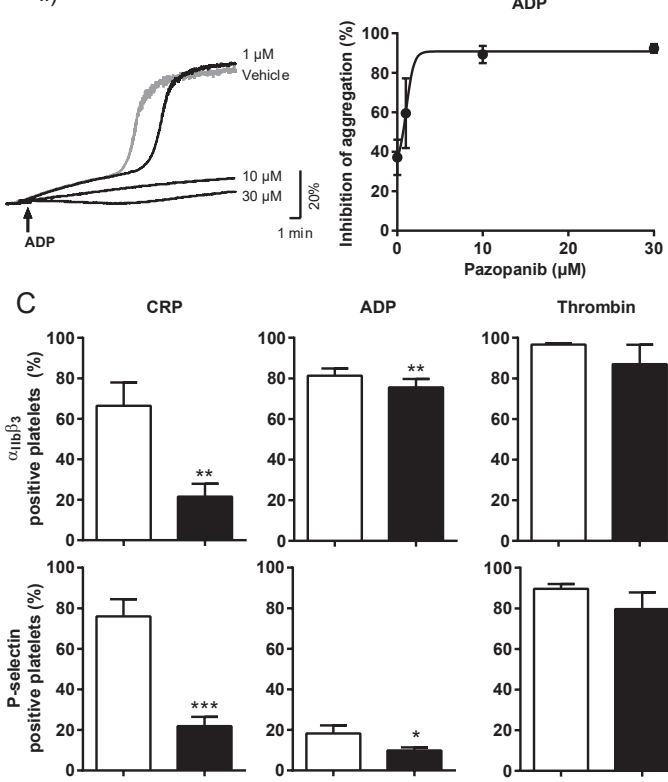

ADP
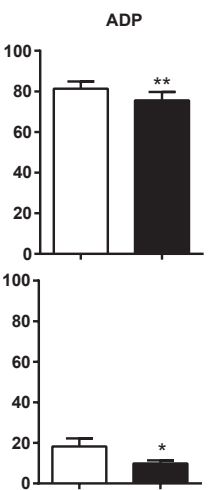

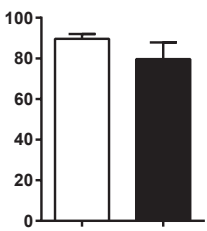

$B$

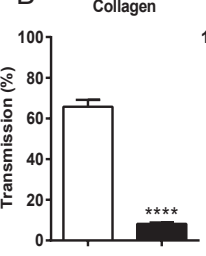

ADP

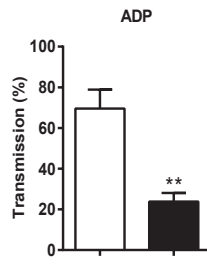

TRAP-6
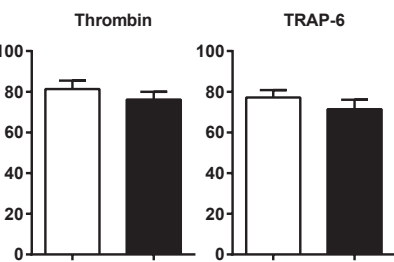

U46619
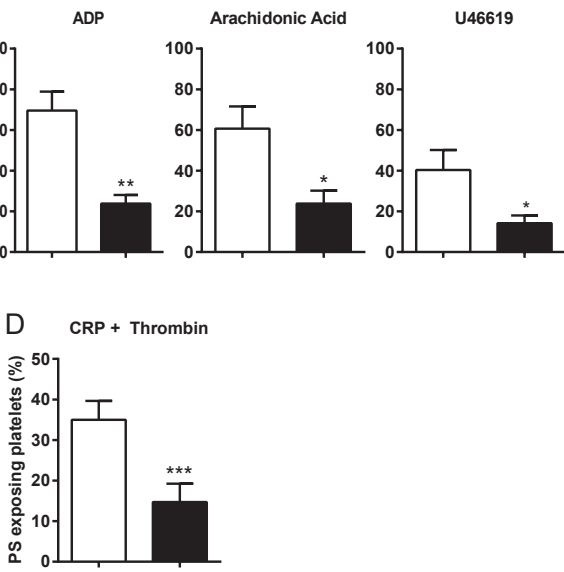

- Vehicle

Pazopanib

Figure 3.1. Pazopanib inhibits collagen- and ADP-induced platelet aggregation, integrin activation, secretion and PS exposure. Washed platelets from healthy donors were incubated with 1, 10, or $30 \mu \mathrm{M}$ pazopanib or vehicle $(0.1 \% \mathrm{DMSO})$ for 10 minutes. (A) Representative aggregation curves and dose-response graphs of inhibition of aggregation $(n=4)$. Arrow indicates addition of (i) $1 \mu \mathrm{g} / \mathrm{mL}$ collagen or (ii) $25 \mu \mathrm{g} / \mathrm{mL}$ fibrinogen and $1 \mu \mathrm{M}$ Me-S-ADP. (B) Aggregation of washed platelets in presence (black bars) or absence (white bars) of $10 \mu \mathrm{M}$ pazopanib was induced by $1 \mu \mathrm{g} / \mathrm{mL}$ collagen, $1 \mathrm{nM}$ thrombin, $10 \mu \mathrm{M}$ TRAP-6, $1 \mu \mathrm{M}$ Me-S-ADP in presence of $25 \mu \mathrm{g} / \mathrm{mL}$ fibrinogen, $30 \mu \mathrm{M}$ arachidonic acid or $1 \mu \mathrm{M} \cup 46619$. Histograms indicate maximal amplitude of aggregation $(n=6)$. (C) Platelets, pre-treated with vehicle or pazopanib $(10 \mu M)$, were stimulated with $5 \mu \mathrm{g} / \mathrm{mL}$ CRP-XL, $1 \mu \mathrm{M}$ Me-S-ADP or $1 \mathrm{nM}$ thrombin for 10-20min, and analysed by flow cytometry (n =6). Shown are percentages of platelets binding FITC-labelled PAC-1 monoclonal antibody against integrin $a_{\| b} b_{3}$ or FITClabelled anti-human CD62 mAb. (D) Platelets pre-treated with vehicle or pazopanib (10 $\mu \mathrm{M})$ were stimulated with $5 \mu \mathrm{g} /$ $\mathrm{mL} C R P-X L$ and $4 \mathrm{nM}$ thrombin for 60 minutes, and analysed by flow cytometry $(n=6)$. Exposure of PS was determined by FITC-labelled annexin A5. Histogram shows percentages of platelets binding FITC-annexin A5. Data are means \pm SEM, $* p<0.05, * * p<0.01, * * * p<0.001, * * * * p<0.0001$. 
an efficient antagonist of platelet responses induced by GPVI agonists (collagen or CRP-XL), but is less effective in antagonizing responses induced by the G-protein coupled receptor agonists ADP, thromboxane $A_{2}$ or thrombin.

\section{Pazopanib inhibits phosphorylation of Syk, and reduces platelet $\mathrm{Ca}^{2+}$ responses}

The decreased platelet responses to GPVI agonists suggested that pazopanib influences platelet signalling via the tyrosine kinase-operating ITAM pathway. To investigate this in more detail, we assessed the effect of pazopanib on protein tyrosine phosphorylation patterns of GPVI-stimulated platelets using western blotting. Pre-incubation of $500 \times 10^{9}$ platelets/L with $30 \mu \mathrm{M}$ pazopanib (i.e., equivalent to $15 \mu \mathrm{M}$ for $250 \times 10^{9}$ platelets/L, used for aggregation experiments) strongly reduced the CRP-XL induced tyrosine phosphorylation of multiple proteins (Figure 3.2A). This suggested interference of pazopanib early in the GPVI signalling cascade. This was confirmed by the finding that pazopanib strongly inhibited the phosphorylation of Syk (Figure 3.2B).

A

A Mwt (kDa)

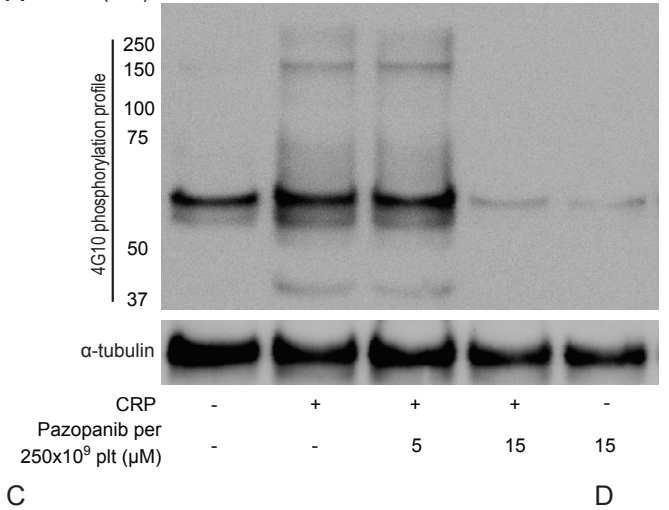

B

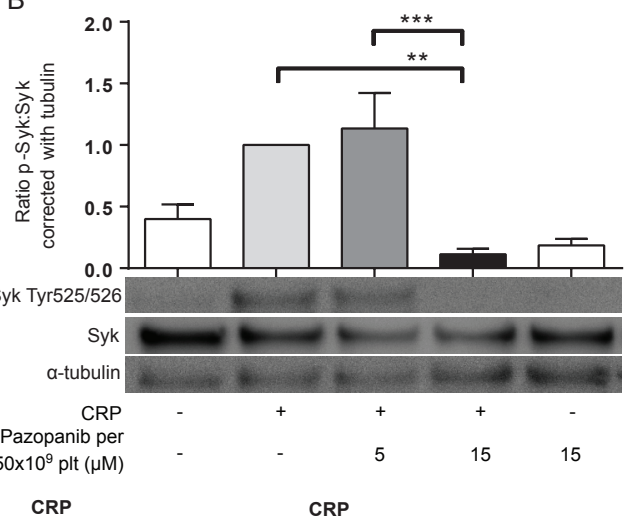

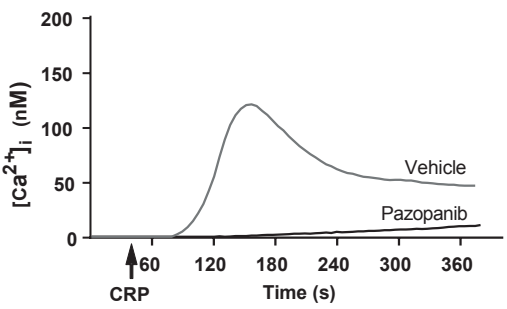
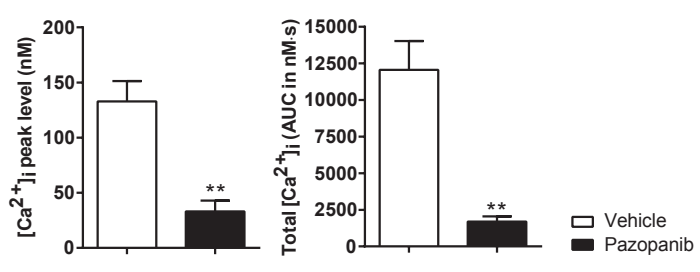

Figure 3.2. Pazopanib inhibits GPVI-induced protein tyrosine phosphorylation via Syk and cytosolic Ca ${ }^{2+}$ rises. $(A, B)$ Representative western blots $(n=4)$ with washed platelets $\left(500 \times 10^{9} / L\right)$ incubated with vehicle or pazopanib $(10$ or $30 \mu \mathrm{M})$ for 10 minutes, and stimulated with $5 \mu \mathrm{g} / \mathrm{mL}$ CRP-XL for 3 minutes. For comparison to Figure 1, pazopanib concentrations were re-calculated to match a platelet concentration of $250 \times 10^{9} / \mathrm{L}$. (A) Protein tyrosine phosphorylation was visualized with 4G10 mAb. Total amount of protein was visualized by staining for a-tubulin. (B) Protein phosphorylation of Syk (Tyr525/526) and Syk, and parallel staining for a-tubulin. Shown is the ratio of phospho-Syk/total Syk assessed by gray intensity analysis $(n=4)$. (C) Representative traces of changes in cytosolic Ca ${ }^{2+}$ of Fura-2-loaded platelets $\left(100 \times 10^{9} / \mathrm{L}\right)$, preincubated with vehicle or $10 \mu \mathrm{M}$ pazopanib for 10 minutes, and stimulated with $1 \mu \mathrm{g} / \mathrm{mL}$ CRP-XL or $1 \mathrm{nM}$ thrombin in the presence of $1 \mathrm{mM} \mathrm{CaCl}_{2}$. (D) Histograms show maximal rise in $\left[\mathrm{Ca}^{2+}\right]_{i}$ and total $\left[\mathrm{Ca}^{2+}\right]_{i}$ (area-under-curve, AUC) $(n=5)$. Data are means $\pm \mathrm{SEM},{ }^{* *} p<0.01, * * * p<0.001$. 
GPVI signalling via Syk results in elevation of intracellular Ca ${ }^{2+}$ levels $\left(\left[\mathrm{Ca}^{2+}\right]_{\mathrm{i}}\right)$ as a prerequisite for integrin activation, secretion and PS exposure. To investigate this, platelets were loaded with the $\mathrm{Ca}^{2+}$ probe Fura-2, and agonist-induced responses were measured. In platelets stimulated with CRP-XL, the presence of pazopanib resulted in a reduction of the maximal (peak height) and total (area-under-curve) $\mathrm{Ca}^{2+}$ rises (Figure 3.2C-D). Pazopanib did however not influence $\left[\mathrm{Ca}^{2+}\right]_{\mathrm{i}}$ elevation in response to thrombin stimulation (not shown). These results confirm that pazopanib interfered in the early GPVI-induced signalling cascade.

\section{Pazopanib affects thrombus size and PS exposure in whole blood under flow}

Platelet activation via GPVI is an essential step in collagen-induced thrombus formation in whole blood under flow conditions ${ }^{41}$. This method was used to determine the effect of pazopanib on platelets in a whole blood environment. Pre-incubation of blood with $30 \mu \mathrm{M}$ pazopanib resulted in a small, but significant decrease in platelet deposition compared to vehicle-treated blood (Figure 3.3A-B). Furthermore, the height of thrombi with multi-layered platelets was reduced with pazopanib (Figure 3.3C). The aggregate-reducing effect was confirmed by measuring the size distribution of thrombi (integrated feature size), which was significantly decreased after treatment of the blood with pazopanib (Figure 3.3D). Post-staining with fluorescently labelled annexin A5 indicated a lower PS exposure (Figure 3.3E), as in agreement with the flow cytometric results using washed platelets.

A
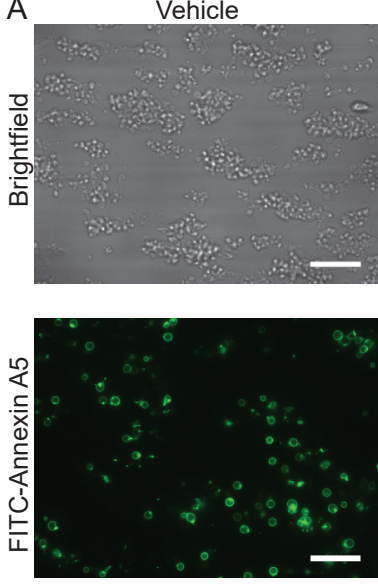

Pazopanib
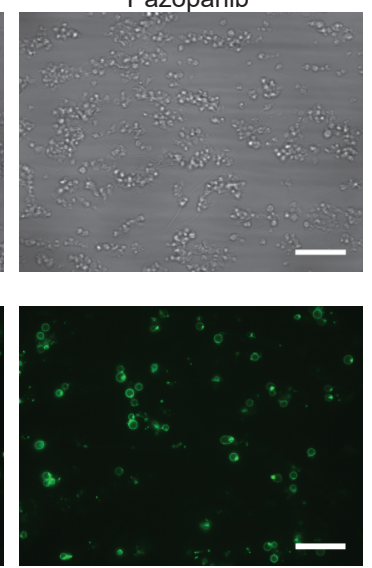

B

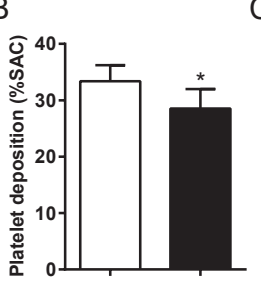

D

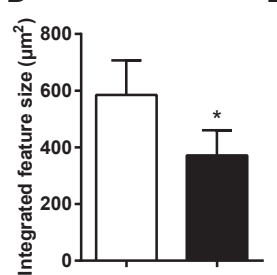

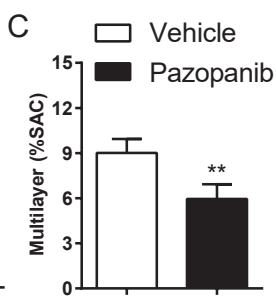

$\mathrm{E}$

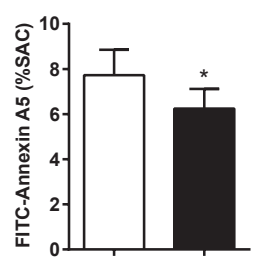

Figure 3.3. Pazopanib suppresses thrombus formation in whole blood perfused over collagen. Whole blood from healthy volunteers was perfused for 4 minutes at wall shear rate of 1,000 $\mathrm{s}^{-1}$ over microspots containing collagen-I. (A) Representative brightfield images (upper panel) and fluorescence images of PS exposure (lower panel) of control blood sample incubated with $30 \mu \mathrm{M}$ pazopanib. Bar=20 $\mu \mathrm{m}$. (B-E) Quantification $(n=6)$ of brightfield images of: platelet deposition (B), multilayered thrombus (C), cumulative size of thrombus (integrated feature size) (D), and fluorescence images of staining for PS exposure (E). Data are means $\pm \operatorname{SEM}(n=6),{ }^{*} p<0.05,{ }^{*} p<0.01$. 


\section{Pazopanib treatment of renal cell carcinoma patients moderately affects platelet functions}

To investigate the clinical relevance of these findings, blood samples were obtained from ten patients diagnosed with metastatic RCC, and eligible for pazopanib treatment (Table 3.1). The patients (6 females) had a mean age of 69 (range: $51-88$ ) years. Blood samples were collected at one day prior and at 14 days after the start of pazopanib treatment. Median platelet count in the patients' blood before start of treatment was $230 \times 10^{9} / \mathrm{L}$, while this moderately, but significantly reduced to $201 \times 10^{9} / \mathrm{L}$ after treatment (Figure 3.4A). Bleeding complications were reported for three patients, who all developed mild epistaxis (Table 3.1).

Using washed platelets from patients before and after pazopanib treatment, aggregation was determined by light transmission aggregometry. Strikingly, no treatment effect could be observed upon collagen stimulation (Figure 3.4B). Flow cytometry was used to assess $\alpha_{\| 1} \beta_{3}$ integrin activation and secretion upon stimulation with CRP-XL, ADP or thrombin. Again, no effect of the treatment on these platelet responses could be observed (Figure 3.4C). On the other hand, platelet procoagulant activity, as determined from PS exposure in response to CRP-XL plus thrombin, was significantly decreased by $18 \%$ after treatment (Figure 3.4D).

In whole blood flow experiments over collagen, parameters of thrombus formation were investigated before and after pazopanib treatment. Whereas platelet deposition and thrombus buildup were not affected in the post-treatment blood samples (Figure 3.5A-D), a significant reduction of $48 \%$ was seen in PS exposure in the post-treatment samples, as compared to pretreatment (Figure 3.5A, E).

\section{Plasma impairs pazopanib effects on platelet function}

In isolated platelets we observed a dose-dependent inhibiting effect of pazopanib on platelet function via the inhibition of tyrosine phosphorylation of Syk, and downstream intracellular $\mathrm{Ca}^{2+}$ signalling. This was accompanied by reduced integrin activation, secretion and PS exposure. In contrast, pazopanib treatment of RCC patients only resulted in reduction of platelet

Table 3.1. Characteristics of patients treated with pazopanib $800 \mathrm{mg} / \mathrm{day}$.

\begin{tabular}{lcccl}
\hline Patient no. & $\begin{array}{c}\text { Age range } \\
\text { (years) }\end{array}$ & $\begin{array}{c}\text { Platelet count } \\
\text { day } 0\left(\times 10^{9} / \text { L) }\right.\end{array}$ & $\begin{array}{c}\text { Platelet count } \\
\text { day } 14\left(\times 10^{9} / \text { L) }\right)\end{array}$ & Bleeding \\
\hline 1 & $56-60$ & 238 & 183 & No \\
2 & $61-65$ & 222 & 212 & No \\
3 & $71-75$ & 175 & 107 & Yes, epistaxis \\
4 & $61-65$ & 212 & 189 & No \\
5 & $66-70$ & 217 & 219 & Yes, epistaxis \\
6 & $81-85$ & 443 & 288 & Yes, epistaxis \\
7 & $66-70$ & 402 & 130 & No \\
8 & $51-55$ & 295 & 249 & No \\
9 & $76-80$ & 274 & 276 & No \\
10 & $81-85$ & 179 & 139 & No \\
\hline
\end{tabular}


PS exposure. We hypothesized that the presence of plasma can interfere with the antiplatelet activity of pazopanib. To investigate this, experiments were performed with both PRP and washed platelets, which were incubated with different concentrations of pazopanib. In undiluted PRP, collagen-induced aggregation was not affected by pazopanib, whereas this response was strongly inhibited at $10 \mu \mathrm{M}$ in washed platelets (Figure 3.6A). Pre-incubation of PRP with increasing doses of pazopanib until $20 \mu \mathrm{M}$ did not inhibit PS exposure, in contrast to pre-incubation of washed platelets (Figure 3.6B).

It has been described that pazopanib is highly bound by plasma proteins at concentration ranges of $10-100 \mu \mathrm{g} / \mathrm{mL}^{24}$. This corresponds with a concentration of 23-230 $\mu \mathrm{M}$. Hence, also higher concentrations of pazopanib $(50-100 \mu \mathrm{M})$ were tested in platelets incubated at different

A

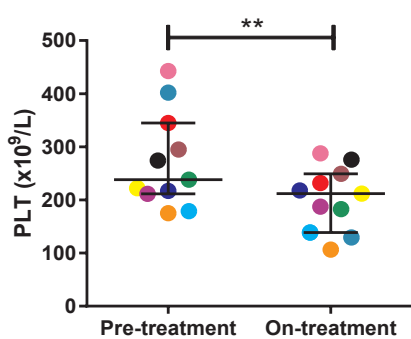

C
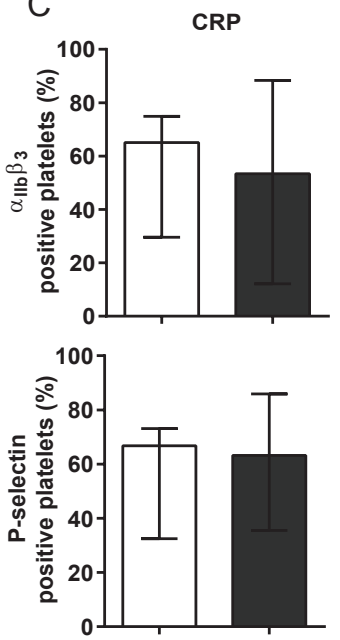

B

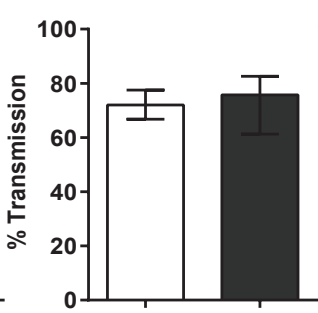

ADP
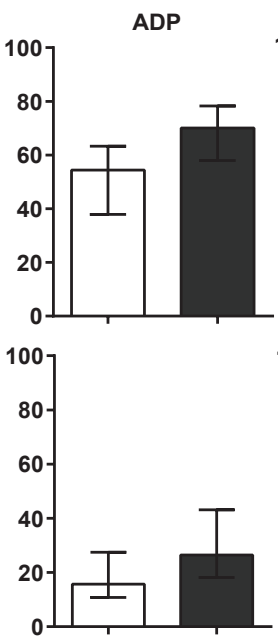

ADP

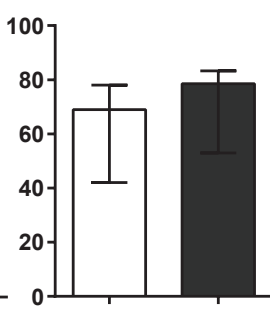

Thrombin

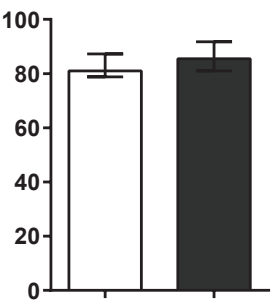

D

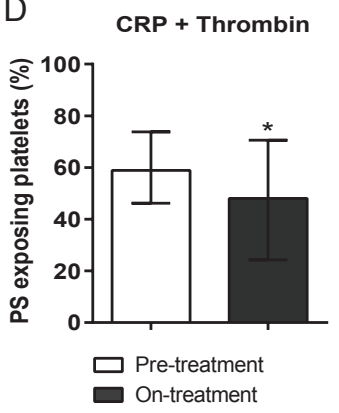

Figure 3.4. Treatment of carcinoma patients with pazopanib decreased platelet count and suppressed phosphatidylserine exposure in washed platelets. Blood from patients was collected the day before and at 2 weeks after pazopanib treatment. (A) Platelet count was measured in blood from RCC patients before and on treatment with pazopanib. Each dot represents a single patient. (B) Platelets were isolated and aggregation was induced by $1 \mu \mathrm{g} / \mathrm{mL}$ collagen, $1 \mu \mathrm{M}$ Me-S-ADP (in the presence of $25 \mu \mathrm{g} / \mathrm{mL}$ fibrinogen), or $1 \mathrm{nM}$ thrombin. Histograms indicate maximal amplitude of aggregation $(n=6)$. (C) Platelets before and during treatment of patients were stimulated with $5 \mu \mathrm{g} / \mathrm{mL}$ CRP$X L, 1 \mu M$ 2Me-S-ADP or $1 \mathrm{nM}$ thrombin for 10-20min, and analyzed by flow cytometry $(n=10)$. Shown are percentages of platelets binding FITC-PAC1 mAb (allbb3 expression) or FITC-anti-CD62P mAb (P-selectin expression). (D) Patient platelets were stimulated with $5 \mu \mathrm{g} / \mathrm{mL} \mathrm{CRP-XL}$ and $4 \mathrm{nM}$ thrombin for $60 \mathrm{~min}$, and analyzed by flow cytometry $(n=6)$. Histogram shows percentages of platelets binding FITC-annexin A5 (PS exposure). Data are medians \pm interquartile ranges, ${ }^{*} p<0.05$, $* * p<0.01$. 

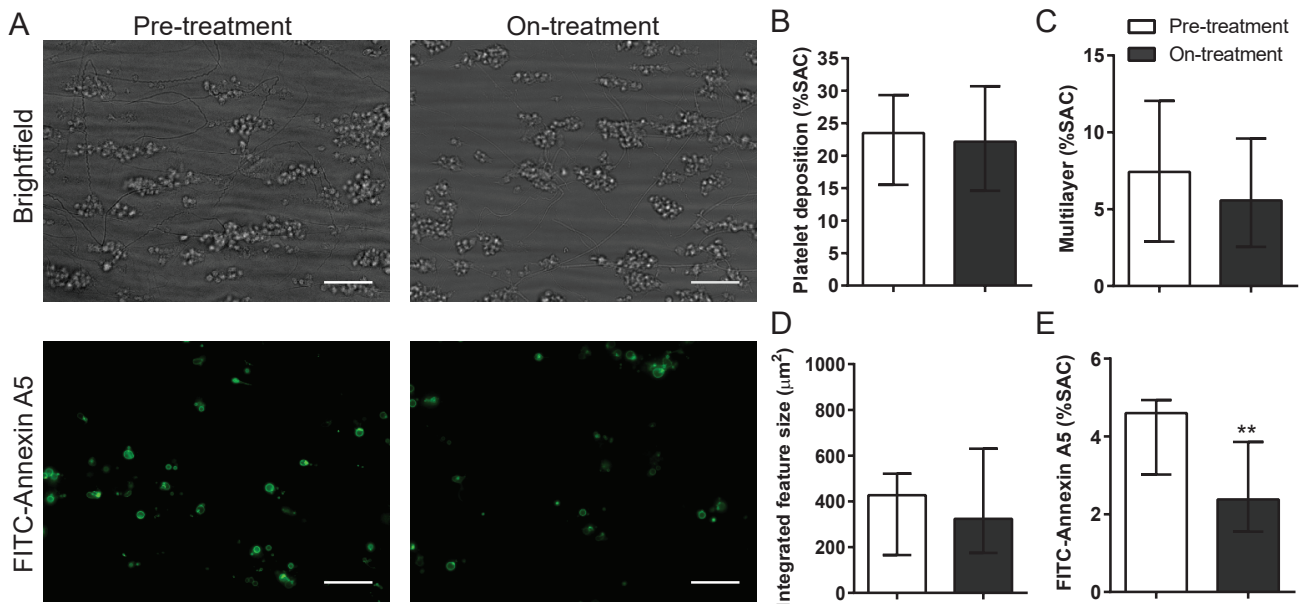

D

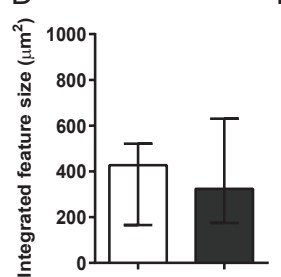

E

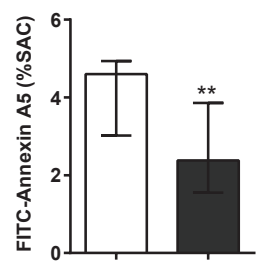

Figure 3.5. Treatment of pazopanib patients suppressed phosphatidylserine exposure upon thrombus formation on collagen. (A) Whole blood from patients was perfused for $4 \mathrm{~min}$ at wall shear rate of $1,000 \mathrm{~s}^{-1}$ over a collagen surface. Shown are representative brightfield images (upper panel) and fluorescence images of PS exposure (lower panel) for blood samples taken before and after 2 weeks of pazopanib treatment. Bar=20 $\mu \mathrm{m}$. (B-E) Quantification of brightfield images of platelet deposition (B), multilayered thrombus (C), cumulative size of thrombus (integrated feature size, IFS) (D), and fluorescence images of staining for PS exposure (E). Data are medians \pm interquartile ranges $(n=10),{ }^{* *} p<0.01$.

A

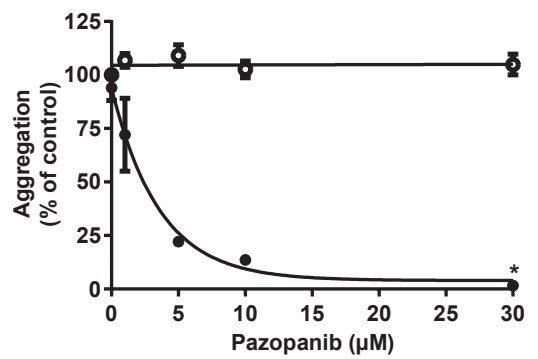

B

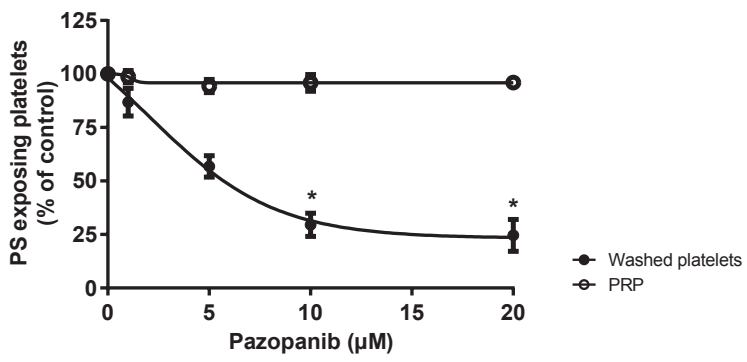

C

CRP + TRAP-6

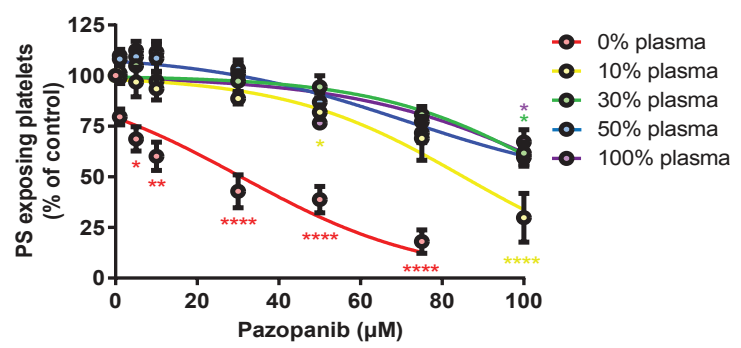

Figure 3.6. Plasma suppresses effects of pazopanib on platelet activation in vitro. Washed platelets or PRP from healthy donors were incubated with 1,5, 10, or $30 \mu \mathrm{M}$ pazopanib or vehicle (0.1\% DMSO) for 10 minutes. (A) Aggregation of platelets in buffer or PRP in response to $1 \mu \mathrm{g} / \mathrm{mL}$ collagen. (B) Exposure of PS measured in washed platelets or in PRP, stimulated with $5 \mu \mathrm{g} / \mathrm{mL}$ CRP-XL and $4 \mathrm{nM}$ thrombin for 60 minutes, and analyzed by flow cytometry using FITC-labeled annexin A5. (C) Platelets reconstituted at different plasma concentrations were pre-incubated with pazopanib (1-100 $\mu \mathrm{M})$, and stimulated with $5 \mu \mathrm{g} / \mathrm{mL}$ CRP-XL plus $15 \mu \mathrm{M}$ TRAP-6; PS exposure was quantified by flow cytometry using FITClabeled annexin A5 (PS exposure). Data are means \pm SEM $(n=3),{ }^{*} p<0.05,{ }^{* *} p<0.01,{ }^{* * * *} p<0.0001$. 


\section{Chapter 3}

plasma concentrations $(0,10,30,50$ or $100 \%$ plasma). It appeared that pazopanib treatment substantially suppressed PS exposure in response to CRP-XL plus TRAP-6 in the presence of low amounts of plasma (Figure 3.6C). However, at $100 \mu \mathrm{M}$ pazopanib, PS exposure was still significantly reduced by $25 \%$ with $30-100 \%$ plasma (Figure 3.6C, $p<0.01$ ). This effect is comparable to the moderate inhibition of PS exposure in patients treated with pazopanib (see Figure 3.4D). These results suggest that the presence of plasma interferes with the incorporation of pazopanib into platelets.

\section{Discussion}

In the present study, we demonstrate that the multi-target TKI pazopanib markedly reduces the collagen-induced activation responses of isolated platelets, including aggregation, PS exposure and $\mathrm{Ca}^{2+}$ signalling through inhibition of tyrosine kinases, including Syk. In blood samples from RCC patients treated with pazopanib, these effects were confined to a suppression of GPVI-mediated PS exposure, observed in isolated platelets as well as in whole blood thrombus formation under flow.

Several protein tyrosine kinases are known to contribute to platelet activation processes and haemostasis ${ }^{8,10,11}$. An activity-based kinase profiling already showed that pazopanib can target various tyrosine kinases that are highly expressed in platelets ${ }^{42}$. Here we confirm that, in washed platelets, the GPVI-dependent phosphorylation of multiple proteins, including Syk, is suppressed by pazopanib, in a way accompanied by reduced platelet activation processes. Syk is known to have multiple sites of phosphorylation which both regulate activity and serve as docking motifs for other proteins ${ }^{43}$. These sites include Tyr-348 and Tyr-352 within the SH2-linker region, Tyr-525 and Tyr-526 within the activation loop of the kinase domain, Tyr-630 in the C terminus of Syk, and other sites such as Thr-384 and Ser-297. It has been shown that Tyr-525/526 is essential for Syk function ${ }^{44}$. Therefore, we selected this phosphorylation site to study the effect of pazopanib on platelet function. However, from the present data it cannot be concluded whether pazopanib inhibits Syk directly, or if its phosphorylation is reduced through inhibition of upstream tyrosine kinases. Most likely, it can be both as pazopanib has been shown to have affinity for multiple TKs in platelets. We have observed that the Syk inhibitor II completely inhibits PS-exposure in washed platelets ${ }^{37}$, which was much stronger than the inhibitory effect of pazopanib (Figure 3.1D). Furthermore deficiency and selective inhibition of Syk has been shown to prevent platelet aggregation and activation in response to collagen-receptor stimulation in mice ${ }^{45}$.

Interestingly, pazopanib also suppressed platelet responses to ADP, arachidonic acid and $\mathrm{TxA}_{2}$ analogue, while responses to thrombin were unaffected. In agreement with this is the recent finding that three tyrosine kinases are phosphorylated upon platelet activation with ADP using phosphoproteomics ${ }^{46}$. These are JAK3, Btk and TNK2, indicating TKs are involved in signalling underneath ADP. Whether TKs are also phosphorylated under AA and thromboxane $A_{2}$ receptor 
stimulation has not yet been investigated with phosphoproteomics, but this is not unlikely. Although it appears that the majority of the targets of pazopanib are underneath GPVI, these data indicate that pazopanib may also target kinase events downstream of other platelet receptors. Moreover, as it has been shown that inhibition of GPVI alone prevents occlusive thrombus formation without causing bleeding ${ }^{47}$, these results support the hypothesis that the targeting of pathways downstream of additional receptors may be responsible for the increased bleeding risk with pazopanib treatment.

Pazopanib is known to have an extremely high plasma protein binding compared to other $\mathrm{TKIs}^{24,48}$. Therefore, we explored if the presence of blood plasma affected its inhibitory effect on platelet responses. Upon increasing plasma concentrations, it appeared that the inhibition of collagen-induced aggregation became lost at pazopanib concentrations up to $100 \mu \mathrm{M}$, whereas the inhibition of PS exposure was still present albeit diminished. Albumin, as a major plasma protein (about $55 \%$ of plasma proteins) present at $35-50 \mathrm{mg} / \mathrm{mL}^{49}$, is considered to be the main pazopanib-binding plasma component ${ }^{50}$. This is in agreement with the residual, but consistent inhibition of PS exposure observed in the platelets from patients treated with pazopanib, as well as in whole blood thrombus formation under flow.

Patients with advanced RCC are commonly treated with $800 \mathrm{mg}$ pazopanib per day. The reported steady state, maximal concentration here is $45 \mu \mathrm{g} / \mathrm{mL}^{51}$, which corresponds to a concentration of $100 \mu \mathrm{M}$. In phase III trials, pazopanib effectively delays disease progression and reduces tumour lesions ${ }^{25}, 52$. In spite of the only partial response rate ${ }^{25}$, the affinity of pazopanib for VEGF and PDGF receptors is relatively high ${ }^{42}$, and likely is higher than that for the intracellular tyrosine kinases implicated in platelet activation. This may explain why the pazopanib dose used for effective cancer treatment does not completely abolish platelet activation processes, but only platelet procoagulant activity (PS exposure), i.e. a response that is most sensitive to inhibition of the cytosolic $\mathrm{Ca}^{2+}$ rises.

We and others ${ }^{24}$ observed a moderate decrease in platelet count upon pazopanib treatment. This by itself is not expected to result in bleeding, with values still within the normal range of $150-400 \times 10^{9}$ platelets $/ L^{53}$. This effect may suggest interference in platelet formation (megakaryocytopoiesis). This has not been reported so far, but there is evidence that megakaryocytic signalling via Src family and Syk kinases is required for megakaryocyte migration, and platelet formation ${ }^{13}$.

The combination of a reduced platelet count and impaired PS exposure may explain the mostly minor bleeding events observed during pazopanib treatment. In the present study, this held for three out of ten patients, all experiencing epistaxis. This number is relatively high compared to a published clinical trial, reporting mild bleeding in only $13 \%$ of the patients ${ }^{24}$. However, we like to note that the power of our study is low. Mild bleeding has also been reported with the use of other TKIs in cancer therapy ${ }^{18,19}$. Ibrutinib - affecting collagen- and von Willebrand factor- 


\section{Chapter 3}

dependent platelet functions ${ }^{54}$ - can cause a risk of mild bleeding in about half of the treated patients, whilst $4-8 \%$ of these experiencing major haemorrhages ${ }^{14}$. Treatment with the drug ponatinib resulted in a prolonged PFA-100 closure times in patients' blood samples, indicating a loss of platelet function ${ }^{15}$. Bleeding occurred here in about $10 \%$ of the patients, who however occasionally used other anticoagulants or antiplatelet drugs ${ }^{55}$. Treatment with the TKI dasatinib was associated with mild thrombocytopenia and an increased risk of bleeding, likely due to combined effects on megakaryocytes and platelets ${ }^{56}$. Also in the latter case, the patients' platelets were less responsive to collagen stimulation, resulting in decreased thrombus formation ${ }^{57}$. To take this further, we recently reviewed how distinct TKIs inhibit platelet activation mechanisms, as well as the clinical consequences of antiplatelet effects due to TKI treatment ${ }^{58}$. Comparison of affinity profiles of TKIs for platelet targets, as well as literature regarding effects on platelet count, platelet function and bleeding, enabled us to distinguish three categories of TKIs. (i) For several TKIs the bleeding tendency is linked to a lowering of platelet count and/or an impairment of platelet function, (ii) other TKIs are predicated to have an antiplatelet effect, although no bleeding side effects have been reported so far, and (iii) for some TKIs no published data on platelets are available.

In comparison to pazopanib, treatment of RCC patients with sunitinib was found to result in a more profound inhibition of platelet activation ${ }^{18}$. Sunitinib is taken up by platelets, and can thus effectively reduce collagen-receptor induced aggregation and thrombus formation ${ }^{18}$. A comparative study of sunitinib versus pazopanib treatment indicated that both drugs provided a progression-free survival benefit when compared to placebo; however, pazopanib had a better safety and quality-of-life profile ${ }^{52}$. Sunitinib causes bleeding in up to $20 \%$ of patients, and lifethreatening bleeding in $3 \%$ of the patients ${ }^{16}$. As indicated above, for pazopanib these numbers are lower, but still non-negligible ${ }^{24}$. Accordingly treatment of metastatic RCC with either pazopanib or sunitinib should be accompanied by special attention of the haemostatic condition, especially when the patients are also treated with antiplatelet drugs.

In summary, the present work demonstrates that platelet treatment with pazopanib in vitro results in strong inhibition of collagen-induced platelet activation, aggregation and PS exposure, whereas pazopanib treatment of RCC patients is restricted to inhibition of the platelet procoagulant activity. In combination with the reduction in platelet count, these effects are likely to contribute to the higher bleeding tendency in pazopanib treated RCC patients. Therefore, antiplatelet effects of TKIs should be taken into account in therapy decisions for patients, especially when prescribed in combination with antiplatelet drugs.

\section{Acknowledgements}

This study was supported by the Maastricht Thrombosis Expertise Centre as part of the Heart and Vascular Centre (HVC) of the MUMC+. 


\section{Author Contributions Statement}

BMET performed experiments, analysed data and wrote manuscript. MN performed experiments and critically reviewed manuscript. SS performed experiments, wrote medical ethical approval and critically reviewed manuscript. AWG provided essential tools, discussed results and critically reviewed manuscript, MGAoE discussed results and critically reviewed manuscript, MJBA included and took care of RCC patients and critically reviewed manuscript, JWMH designed and discussed experiments, critically reviewed and revised manuscript, MJEK designed and performed experiments, discussed results, wrote and revised manuscript.

\section{Conflict of Interest Statement}

The authors declare no personal, professional or financial relationships that could potentially be construed as a conflict of interest.

\section{References}

1. Jorg Thomas, H., et al., Tyrosine Kinase Inhibitors - A Review on Pharmacology, Metabolism and Side Effects. Cur Drug Metab, 2009. 10(5): p. 470-481.

2. Natoli, C., et al., Tyrosine Kinase Inhibitors. Curr Canc Drug Targets, 2010. 10(5): p. 462-483.

3. Broekman, F., E. Giovannetti, and G.J. Peters, Tyrosine kinase inhibitors: Multi-targeted or singletargeted? World J Clin Oncol, 2011. 2(2): p. 80-93.

4. Gotink, K.J. and H.M. Verheul, Anti-angiogenic tyrosine kinase inhibitors: what is their mechanism of action? Angiogenesis, 2010. 13(1): p. 1-14.

5. Rovithi, M., et al., Alternative scheduling of pulsatile, high dose sunitinib efficiently suppresses tumor growth. J Exp Clin Cancer Res, 2016. 35(1): p. 138.

6. Griffioen, A.W. and G. Molema, Angiogenesis: potentials for pharmacologic intervention in the treatment of cancer, cardiovascular diseases, and chronic inflammation. Pharmacol Rev, 2000. 52(2): p. 237-68.

7. Gross, S., et al., Targeting cancer with kinase inhibitors. J Clin Invest, 2015. 125(5): p. 1780-1789.

8. Watson, S.P., et al., GPVI and integrin alphallb beta3 signaling in platelets. J Thromb Haemost, 2005. 3(8): p. 1752-62.

9. Furman, M.I., et al., Platelet tyrosine kinases and fibrinogen receptor activation. Circ Res, 1994. 75(1): p. 172-80.

10. Senis, Y.A., A. Mazharian, and J. Mori, Src family kinases: at the forefront of platelet activation. Blood, 2014. 124(13): p. 2013-2024.

11. Nieswandt, B. and S.P. Watson, Platelet-collagen interaction: is GPVI the central receptor? Blood, 2003. 102(2): p. 449-61.

12. Gross, B.S., S.K. Melford, and S.P. Watson, Evidence that phospholipase C-gamma2 interacts with SLP-76, Syk, Lyn, LAT and the Fc receptor gamma-chain after stimulation of the collagen receptor glycoprotein VI in human platelets. Eur J Biochem, 1999. 263(3): p. 612-23.

13. Mazharian, A., et al., Critical role of Src-Syk-PLC\{gamma\}2 signaling in megakaryocyte migration and thrombopoiesis. Blood, 2010. 116(5): p. 793-800.

14. Shatzel, J.J., et al., Ibrutinib-associated bleeding: pathogenesis, management and risk reduction strategies. J Thromb Haemost, 2017. 15(5): p. 835-847.

15. Neelakantan, P., et al., Platelet dysfunction associated with ponatinib, a new pan BCR-ABL inhibitor with efficacy for chronic myeloid leukemia resistant to multiple tyrosine kinase inhibitor therapy. Haematologica, 2012. 97(9): p. 1444.

16. Je, Y., F.A. Schutz, and T.K. Choueiri, Risk of bleeding with vascular endothelial growth factor receptor tyrosine-kinase inhibitors sunitinib and sorafenib: a systematic review and meta-analysis of clinical 
trials. Lancet Oncol, 2009. 10(10): p. 967-74.

17. Rigg, R.A., et al., Oral administration of Bruton's tyrosine kinase inhibitors impairs GPVI-mediated platelet function. Am J Physiol Cell Physiol, 2016. 310(5): p. C373-80.

18. Sabrkhany, S., et al., Sunitinib uptake inhibits platelet function in cancer patients. Eur J Cancer, 2016. 66: p. 47-54.

19. Levade, M., et al., Targeting Kinases in Cancer Therapies: Adverse Effects on Blood Platelets. Curr Pharm Des, 2016. 22(16): p. 2315-22.

20. Hamilton, Z., et al., Change in platelet count as a prognostic indicator for response to primary tyrosine kinase inhibitor therapy in metastatic renal cell carcinoma. BJU Int, 2016. 118(6): p. 927-934.

21. Crist, M., et al., Examining the bleeding incidences associated with targeted therapies used in metastatic renal cell carcinoma. Crit Rev Oncol Hematol, 2017. 120: p. 151-162.

22. Kostos, L., et al., Gastrointestinal bleeding in a chronic myeloid leukaemia patient precipitated by dasatinib-induced platelet dysfunction: Case report. Platelets, 2015. 26(8): p. 809-11.

23. Bukowski, R.M., U. Yasothan, and P. Kirkpatrick, Pazopanib. Nat Rev Drug Discov, 2010. 9(1): p. 17-18.

24. McCormack, P.L., Pazopanib: a review of its use in the management of advanced renal cell carcinoma. Drugs, 2014. 74(10): p. 1111-25.

25. Sternberg, C.N., et al., Pazopanib in Locally Advanced or Metastatic Renal Cell Carcinoma: Results of a Randomized Phase III Trial. J Clin Oncol, 2010. 28(6): p. 1061-1068.

26. Fabian, M.A., et al., A small molecule-kinase interaction map for clinical kinase inhibitors. Nat Biotechnol, 2005. 23(3): p. 329-36.

27. Karaman, M.W., et al., A quantitative analysis of kinase inhibitor selectivity. Nat Biotechnol, 2008. 26(1): p. 127-32.

28. Mattheij, N.J., et al., Dual mechanism of integrin alphallbbeta3 closure in procoagulant platelets. J Biol Chem, 2013. 288(19): p. 13325-36.

29. Strehl, A., et al., Dual role of platelet protein kinase $\mathrm{C}$ in thrombus formation: stimulation of proaggregatory and suppression of procoagulant activity in platelets. J Biol Chem, 2007. 282(10): p. 7046-55.

30. Baaten, C.C., et al., Gradual increase in thrombogenicity of juvenile platelets formed upon offset of prasugrel medication. Haematologica, 2015. 100(9): p. 1131-8.

31. van Kruchten, R., et al., Both TMEM16F-dependent and TMEM16F-independent pathways contribute to phosphatidylserine exposure in platelet apoptosis and platelet activation. Blood, 2013. 121(10): p. 1850-7.

32. De Witt, S.M., et al., Identification of platelet function defects by multi-parameter assessment of thrombus formation. Nat Commun, 2014. 5: p. 4257.

33. Van Kruchten, R., J.M. Cosemans, and J.W. Heemskerk, Measurement of whole blood thrombus formation using parallel-plate flow chambers- a practical guide. Platelets, 2012. 23(3): p. 229-42.

34. De Witt, S., et al., Multi-parameter assessment of thrombus formation on microspotted arrays of thrombogenic surfaces. Nat Prot Exchange, 2014.

35. Feijge, M.A., et al., Inter-individual variability in $\mathrm{Ca} 2+$ signalling in platelets from healthy volunteers: effects of aspirin and relationship with expression of endomembrane Ca2+-ATPases. Br J Haematol, 1998. 102(3): p. 850-9.

36. Heemskerk, J.W., et al., The Ca2+-mobilizing potency of alpha-thrombin and thrombin-receptoractivating peptide on human platelets-- concentration and time effects of thrombin-induced $\mathrm{Ca} 2+$ signaling. Eur J Biochem, 1997. 249(2): p. 547-55.

37. Van der Meijden, P.E., et al., Key role of integrin alpha(Ilb)beta (3) signaling to Syk kinase in tissue factor-induced thrombin generation. Cell Mol Life Sci, 2012. 69(20): p. 3481-92.

38. Versteeg, H.H., et al., New fundamentals in hemostasis. Physiol Rev, 2013. 93(1): p. 327-58.

39. Munnix, I.C., et al., Segregation of platelet aggregatory and procoagulant microdomains in thrombus formation: regulation by transient integrin activation. Arterioscler Thromb Vasc Biol, 2007. 27(11): p. 2484-90.

40. Kuijpers, M.J., et al., Key role of platelet procoagulant activity in tissue factor-and collagen-dependent thrombus formation in arterioles and venules in vivo differential sensitivity to thrombin inhibition. Microcirculation, 2008. 15(4): p. 269-82.

41. Nieswandt, B., et al., Glycoprotein VI but not alpha2beta1 integrin is essential for platelet interaction 
with collagen. Embo j, 2001. 20(9): p. 2120-30.

42. Kitagawa, D., et al., Activity-based kinase profiling of approved tyrosine kinase inhibitors. Genes Cells, 2013. 18(2): p. 110-22.

43. Sada, K., et al., Structure and function of Syk protein-tyrosine kinase. J Biochem, 2001. 130(2): p. 177-86.

44. Zhang, J., et al., Phosphorylation of Syk activation loop tyrosines is essential for Syk function. An in vivo study using a specific anti-Syk activation loop phosphotyrosine antibody. J Biol Chem, 2000. 275(45): p. 35442-7.

45. van Eeuwijk, J.M., et al., The Novel Oral Syk Inhibitor, Bl1002494, Protects Mice From Arterial Thrombosis and Thromboinflammatory Brain Infarction. Arterioscler Thromb Vasc Biol, 2016. 36(6): p. 1247-53.

46. Beck, F., et al., Temporal quantitative phosphoproteomics of ADP stimulation reveals novel central nodes in platelet activation and inhibition. Blood, 2017. 129(2): p. e1-e12.

47. Andrews, R.K., J.F. Arthur, and E.E. Gardiner, Targeting GPVI as a novel antithrombotic strategy. J Blood Med, 2014. 5: p. 59-68.

48. O'Brien, Z. and M.F. Moghaddam, A Systematic Analysis of Physicochemical and ADME Properties of All Small Molecule Kinase Inhibitors Approved by US FDA from January 2001 to October 2015. Curr Med Chem, 2017. 24(29): p. 3159-3184.

49. Anderson, N.L. and N.G. Anderson, The human plasma proteome: history, character, and diagnostic prospects. Mol Cell Proteomics, 2002. 1(11): p. 845-67.

50. Imbs, D.C., et al., Determination of unbound fraction of pazopanib in vitro and in cancer patients reveals albumin as the main binding site. Invest New Drugs, 2016. 34(1): p. 41-8.

51. Hurwitz, H.I., et al., Phase I trial of pazopanib in patients with advanced cancer. Clin Cancer Res, 2009. 15(12): p. 4220-7.

52. Motzer, R.J., et al., Pazopanib versus sunitinib in metastatic renal-cell carcinoma. N Engl J Med, 2013. 369(8): p. 722-31.

53. Giles, C., The platelet count and mean platelet volume. Br J Haematol, 1981. 48(1): p. 31-7.

54. Levade, M., et al., Ibrutinib treatment affects collagen and von Willebrand factor-dependent platelet functions. Blood, 2014. 124(26): p. 3991.

55. Nazha, A., et al., The clinical impact of ponatinib on the risk of bleeding in patients with chronic myeloid leukemia. Haematologica, 2013. 98(10): p. e131-e131.

56. Mazharian, A., et al., Dasatinib enhances megakaryocyte differentiation but inhibits platelet formation. Blood, 2011. 117(19): p. 5198-5206.

57. Gratacap, M.-P., et al., The new tyrosine-kinase inhibitor and anticancer drug dasatinib reversibly affects platelet activation in vitro and in vivo. Blood, 2009. 114(9): p. 1884.

58. Tullemans, B.M.E., J.W.M. Heemskerk, and M.J.E. Kuijpers, Acquired platelet antagonism: off-target antiplatelet effects of malignancy treatment with tyrosine kinase inhibitors. J Thromb Haemost, 2018. 16:1686-1699. 

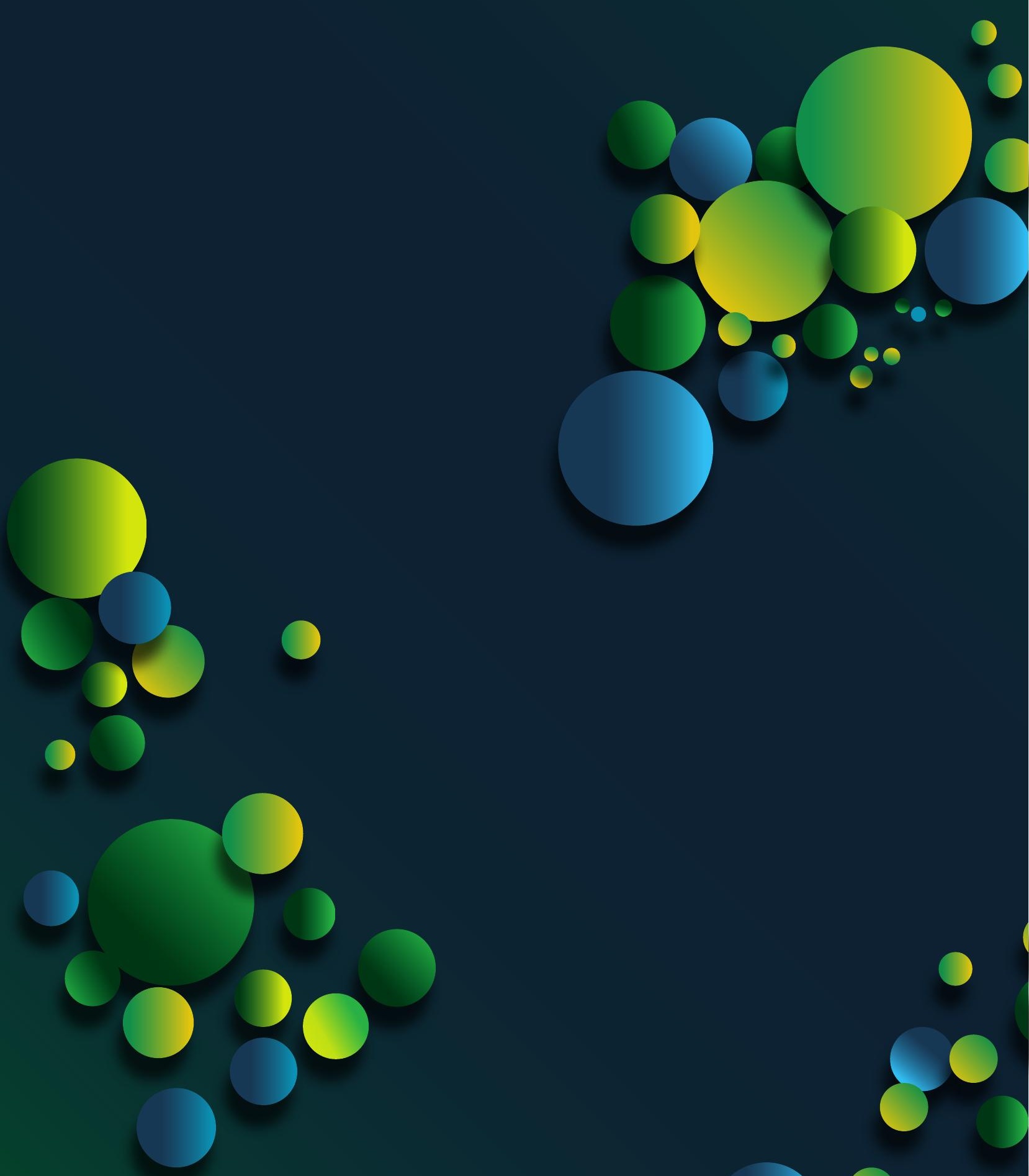


\section{Chapter 4}

Tyrosine kinase inhibitor sunitinib delays platelet-induced coagulation: additive effects of aspirin

Tullemans BME, Fernández DI*, Veninga A*, Baaten CCFMJ, Peters LJF, Aarts MJB, Eble JA, Campello E, Spiezia L, Simioni $P$, van der Vorst EPC, van der Meijden PEJ, Heemskerk JWM and Kuijpers MJE (* equal contribution)

Thromb Haemost 2021; Online ahead of print Reprinted with permission 


\section{Abstract}

Background: Sunitinib is a multi-target tyrosine kinase inhibitor (TKI) used for cancer treatment. In platelets, sunitinib affects collagen-induced activation under non-coagulating conditions. We investigated (i) the effects of sunitinib on thrombus formation induced by other TK-dependent receptors, and (ii) the effects under coagulating conditions. Cardiovascular disease is a co-morbidity in cancer patients, resulting in possible aspirin treatment. Sunitinib and aspirin are associated with increased bleeding risk, and therefore we also investigated (iii) the synergistic effects of these compounds on thrombus and fibrin formation.

Methods: Blood or isolated platelets from healthy volunteers or cancer patients were incubated with sunitinib and/or aspirin or vehicle. Platelet activation was determined by TK phosphorylation, flow cytometry, changes in $\left[\mathrm{Ca}^{2+}\right]_{\mathrm{i}}$, aggregometry and whole blood perfusion over multiple surfaces, including collagen with(out) tissue factor (TF) was performed.

Results: Sunitinib reduced thrombus formation and phosphatidylserine (PS) exposure under flow on collagen type I and III. Also, sunitinib inhibited GPVI-induced TK phosphorylation and $\mathrm{Ca}^{2+}$ elevation. Upon TF-triggered coagulation, sunitinib decreased PS exposure and fibrin formation. In blood from cancer patients more pronounced effects of sunitinib were observed in lung and pancreatic as compared to neuroglioblastoma and other cancer types. Compared to sunitinib alone, sunitinib plus aspirin further reduced platelet aggregation, thrombus formation and PS exposure on collagen under flow with(out) coagulation.

Conclusions: Sunitinib suppresses collagen-induced procoagulant activity and delays fibrin formation, which was aggravated by aspirin. Therefore, we urge for awareness of the combined antiplatelet effects of TKIs with aspirin, as this may result in increased risk of bleeding.

\section{Visual abstract}

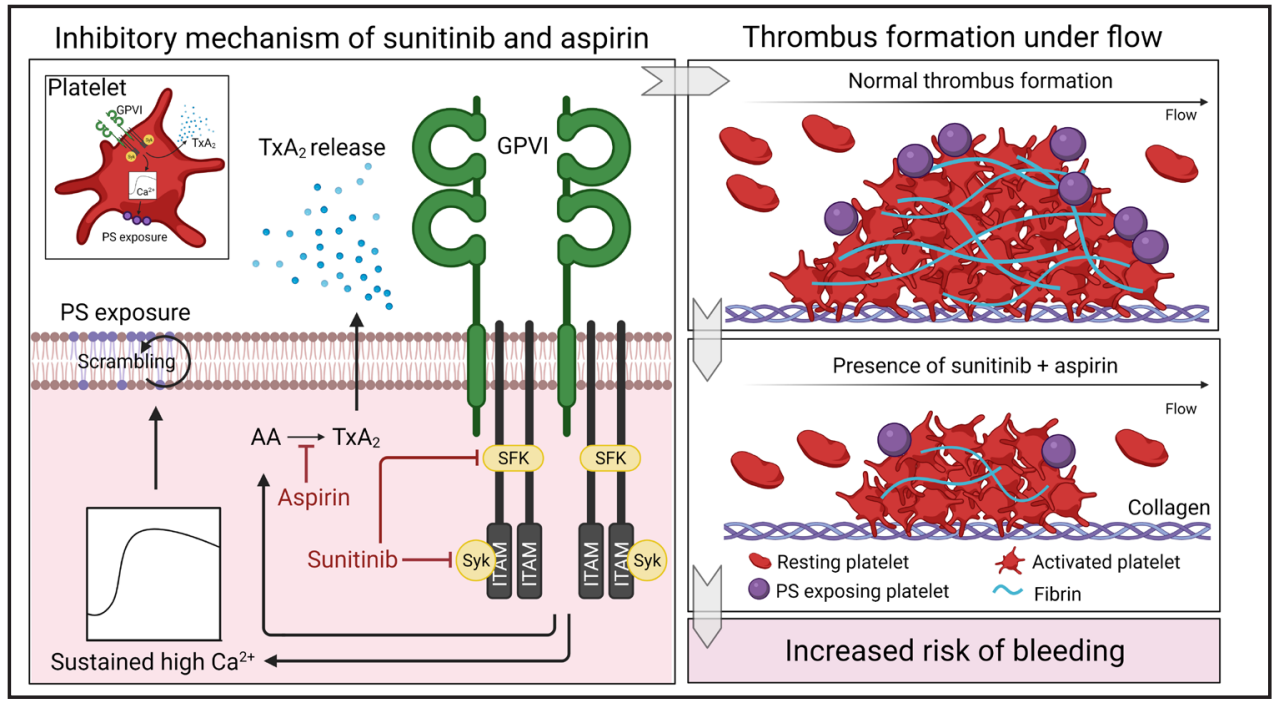




\section{Introduction}

Treatment of cancer patients with sunitinib results in anticancer effects and improves progression free survival ${ }^{1,2}$. Sunitinib is a broad-spectrum tyrosine kinase inhibitor (TKI), affecting not only tumour cells, but also the blood vessel wall ${ }^{3}$. A side effect of sunitinib treatment is mild bleeding (mainly epistaxis, and mucocutaneous and gastrointestinal bleeding, which occurs in approximately $19 \%$ of sunitinib-treated patients) ${ }^{3}$. Fortunately, in the majority of cases, the effects on haemostasis remain sub-clinical.

Platelets contribute to haemostasis by signalling pathways involving multiple protein tyrosine kinases (TKs) such as Syk, Btk and Src family kinases (SFKs) ${ }^{4}$. We and other authors have previously shown that sunitinib is sequestered by platelets, which affects collagen-induced platelet function under non-coagulating conditions ${ }^{5,6}$. TKs do not only signal under the collagen receptor glycoprotein (GP)VI, but also under the receptors GPIb, CLEC-2 and integrins $\alpha_{6} \beta_{1}$ and $\alpha_{\| 1} \beta_{3}$ that bind von Willebrand factor (VWF), podoplanin, laminin and fibrinogen, respectively ${ }^{4}$. The effects of sunitinib on thrombus formation stimulated via these receptors has not been studied. Furthermore, the question arises whether the suppression of collagen-induced thrombus formation is also present under physiological conditions of coagulation.

The link between cancer and arterial thrombosis has been well-established ${ }^{7,8}$. Cancer patients with a cardiovascular history receive treatment with anti-platelet drugs like aspirin to prevent recurrent events. Aspirin irreversibly inhibits platelet activation and is associated with an increased bleeding risk ${ }^{9}$ by preventing the formation of thromboxane $A_{2}\left(T x A_{2}\right)^{10}$. TxA 2 acts as an important soluble agonist released by platelets upon GPVI stimulation. In this respect, aspirin and other platelet-targeted pharmacologic treatments are under investigation as adjuvant anti-cancer therapy ${ }^{11,12}$. The combined effects of these drugs on platelet function have not been investigated thus far.

The aim of the present study was to further investigate the effect of sunitinib on thrombus formation by stimulation with multiple agonists, as well as under coagulating conditions stimulated by collagen plus TF. Furthermore, we assessed whether treatment with sunitinib in combination with aspirin increasingly inhibited collagen-induced thrombus formation as compared to either compound alone. The results indeed show an enhancing effect of this dual treatment of platelets with sunitinib and aspirin on collagen-induced platelet phosphatidylserine (PS) exposure and ensuing fibrin formation. Finally, platelet-dependent fibrin formation in whole blood from cancer patients was significantly inhibited by sunitinib.

\section{Materials and Methods}

Materials

Sunitinib malate (Sutent) was provided by Pfizer (New York NY, USA). Aspirin, bovine serum albumin (BSA), D(+)-glucose, unfractionated heparin, and apyrase were purchased from 


\section{Chapter 4}

Sigma-Aldrich (Saint Louis MO, USA). The agonists collagen-related peptide crosslinked (CRP-XL) and Von Willebrand factor III (VWF-III) were obtained from CambCol Laboratories (Cambridge, UK), whereas thrombin was obtained from Enzyme Research Laboratories (South Bend IN, USA) and Horm collagen type I from Takeda (Hoofddorp, The Netherlands). 2-Methylthio-adenosinediphosphate (2MeS-ADP) and D-phenylalanyl-prolyl-arginyl chloromethyl ketone (PPACK) were obtained from Santa Cruz Biotechnology (Dallas TX, USA). Recombinant human TF (Innovin) was purchased from Dade Behring (Deerfield IL, USA). Laminin came from Octapharma (Berlin, Germany). Fura-2-AM was obtained from Invitrogen (Carlsbad CA, USA). Plastic syringes and fluorescein isothiocyanate (FITC)-labelled PAC1 monoclonal antibody (mAb) against activated human integrin $\alpha_{\| 1 b} \beta_{3}$ were purchased from BD Bioscience (nr. 340507; Franklin Lakes NJ, USA), while FITC-conjugated $\alpha$-fibrinogen mAb was purchased from DAKO (F0111; Santa Clara CA, USA). Alexa Fluor (AF) 647-labelled CD62-P mAb was obtained from Biolegend (London, UK), whereas the FITC-labelled anti-human CD62-P mAb was obtained from Beckman Coulter (nr. 65050; Sydney, Australia). 3,3-dihexyloxacarbocyanine iodide (DiOC6) was purchased from Anaspec (Reeuwijk, The Netherlands). FITC-conjugated annexin A5 was from Pharmatarget (Maastricht, The Netherlands). AF568-conjugated annexin A5 and AF647-labelled fibrinogen were purchased from Molecular Probes, Life Technologies (New York NY, USA).

\section{Blood collection and platelet isolation}

With approval from the medical ethics committee from the Maastricht University Medical Centre+ (MUMC+) and informed consent in accordance with the Declaration of Helsinki, blood was collected from healthy volunteers. Furthermore, blood was collected from 11 patients diagnosed with several cancer types (lung, pancreas, neuroglioblastoma, ovarian or paraganglioma) at the University Hospital of Padua (Italy), also with approval of the local medical ethical committee and after informed consent. Blood was collected in 3.2\% trisodium citrate tubes by venipuncture, after discarding the first $3 \mathrm{~mL}$ of blood. Platelets, plasma or serum were isolated from whole blood as described previously ${ }^{13}$. Whole blood or isolated platelets were incubated with either vehicle or sunitinib (10 or $30 \mu \mathrm{M}$ as indicated) for 10 minutes at room temperature (unless stated otherwise) before measurements.

\section{Flow cytometry}

Washed platelets $\left(100 \times 10^{9}\right.$ platelets/L) were supplemented with $2 \mathrm{mM} \mathrm{CaCl}_{2}$ and stimulated by a combination of CRP-XL $(5 \mu \mathrm{g} / \mathrm{mL})$ and thrombin $(4 \mathrm{nM})$ for 1 hour at $37^{\circ} \mathrm{C}$. Phosphatidyl-serine (PS) exposing platelets were labelled with FITC-conjugated annexin A5 and measured by flow cytometry using a BD Accuri C6TM flow cytometer and accompanying software (Erembodegem, Belgium). 


\section{Cytosolic $\mathrm{Ca}^{2+}$ measurements}

Cytosolic $\mathrm{Ca}^{2+}\left(\left[\mathrm{Ca}^{2+}\right]_{\mathrm{i}}\right)$ measurements in Fura-2 loaded platelets $\left(200 \times 10^{9}\right.$ platelet/L) were performed as described using a Shimadzo RF-5001PC spectrofluorophotometer (Kyoto, Japan) ${ }^{14}$. Levels of $\left[\mathrm{Ca}^{2+}\right]_{i}$ were determined by the conversion of ratio values ${ }^{15}$ with correction for background fluorescence and maximal values of $\left[\mathrm{Ca}^{2+}\right]_{i}$ (peak level) were used as output.

\section{Light transmission aggregometry}

Washed platelets or PRP $\left(250 \times 10^{9}\right.$ platelets/L) were incubated with vehicle or sunitinib (10 or $30 \mu \mathrm{M}$ as indicated) for 10 minutes at $37^{\circ} \mathrm{C}$. Where indicated, aspirin (100 $\left.\mu \mathrm{M}\right)$ was incubated in PRP for 30 minutes at $37^{\circ} \mathrm{C}$ before further platelet isolation. Platelet aggregation was induced by collagen type I $(5 \mu \mathrm{g} / \mathrm{mL})$, 2MeS-ADP $(1 \mu \mathrm{M})$ or thrombin (1 $\mathrm{nM})$ and aggregation responses were measured using a Chronolog optical aggregometer (Havertown PA, USA) and maximum amplitude was quantified.

\section{Pam-Gene kinase assay}

Washed platelets $\left(500 \times 10^{9}\right.$ platelets/L) were pretreated with vehicle (control) or $30 \mu \mathrm{M}$ sunitinib for 10 minutes at $37^{\circ} \mathrm{C}$ and were subsequently stimulated with $5 \mu \mathrm{g} / \mathrm{mL} \mathrm{CRP-XL}$ in the presence of $2 \mathrm{mM} \mathrm{CaCl}_{2}$. Unstimulated, resting platelets served as control. After 90 seconds of stimulation, samples were lysed by adding 1:1 M-PER Mammalian Extraction Buffer containing Halt Phosphatase Inhibitor and EDTA-free Halt Protease Inhibitor Cocktail (1:100 each; Thermo Fischer Scientific). Samples were lysed for 15 minutes on ice and afterwards centrifuged for 15 minutes at $10,000 \mathrm{~g}$ at $4^{\circ} \mathrm{C}$. Supernatants were collected and protein content was quantified with a BioRad DC protein kit (Hercules CA, USA).

Tyrosine kinase profiles were determined using the PamChip ${ }^{\circledR}$ peptide tyrosine kinase microarray system on PamStation ${ }^{\circledR} 12$ (PTK; PamGene International, 's-Hertogenbosch, The Netherlands). Each PTK-PamChip ${ }^{\circledR}$ array contains 144 individual phospho-site(s) that are peptide sequences derived from substrates for TKs. Each peptide on the chip builds a 15-amino acid sequence representing a putative endogenous phosphorylation site which functions as a TK substrate. The phosphorylation of the peptides is visualized by detection of the fluorescent signal which is emitted as a result of the binding of the FITC-conjugated PY2O anti-phosphotyrosine antibody.

For the PTK assay, $7.5 \mu \mathrm{g}$ of protein was applied per array ( $\mathrm{N}=3$ per condition) and carried out using the standard protocol supplied by Pamgene. All reagents used for PTK activity profiling were supplied by Pamgene International B.V. Initially, to prepare the PTK Basic Mix, the freshly frozen lysate was added to $4 \mu \mathrm{L}$ of $10 x$ protein PTK reaction buffer (PK), $0.4 \mu \mathrm{L}$ of 100x BSA, $0.4 \mu \mathrm{L}$ of $1 \mathrm{M}$ dithiothreitol (DTT) solution, $4 \mu \mathrm{L}$ of $10 \mathrm{x}$ PTK additive, $4 \mu \mathrm{L}$ of $4 \mathrm{mM}$ ATP and $0.6 \mu \mathrm{L}$ of monoclonal anti-phosphotyrosine FITC-conjugate detection antibody (clone PY20). Total volume 
of the PTK Basic Mix was adjusted to $40 \mu \mathrm{L}$ by adding distilled water $\left(\mathrm{H}_{2} \mathrm{O}\right)$. Before loading the PTK Basic Mix on the array, a blocking step was performed applying $30 \mu \mathrm{L}$ of $2 \%$ BSA to the middle of every array and washing with PTK solution for PamChip ${ }^{\circledR}$ preprocessing. Next, $40 \mu \mathrm{L}$ of PTK Basic Mix were applied to each array of the PamChips ${ }^{\circledR}$. Then, the microarray assays were run for 94 cycles. An image was recorded by a CCD camera PamStation ${ }^{\circledR} 12$ at kinetic read cycles 32-93 at 10,50 and $200 \mathrm{~ms}$ and at end-level read cycle at 10, 20, 50, 100 and $200 \mathrm{~ms}$. The spot intensity at each time point was quantified (and corrected for local background) using the BioNavigator software version 6.3 (PamGene International, 's-Hertogenbosch, The Netherlands). Upstream Kinase Analysis (UKA) ${ }^{16}$, a functional scoring method (PamGene) was used to rank kinases based on combined specificity scores (based on peptides linked to a kinase, derived from 6 databases) and sensitivity scores (based on treatment-control differences).

\section{Whole blood perfusion experiments with(out) coagulation and quantification}

Whole blood perfusion experiments without coagulation were performed as described before ${ }^{17}$. Platelet activation properties were determined by staining for integrin activation, P-selectin expression and PS exposure using FITC-conjugated $\alpha$-fibrinogen mAb (1:80), AF647conjugated CD62-P (1:100), and AF568-conjugated annexin A5 (1:200).

Whole blood perfusion experiments under coagulating conditions were performed as described before ${ }^{18}$. Platelet activation properties were determined by supplementing blood samples with AF647-fibrinogen (16.5 $\mathrm{gg} / \mathrm{mL}$ f.c), DiOC6 (0.5 $\mu \mathrm{g} / \mathrm{mL}$ f.c.), and AF568-Annexin A5 $(1: 200)$.

Image capturing was performed using an EVOS microscope (Life Technologies, Carlsbad CA, USA). All images were analysed using specific (half-automated) scripts in the open-access Fiji software (Laboratory for Optical and Computational Instrumentation, University of WisconsinMadison WI, USA) as described ${ }^{17}$. For comparative data analysis heatmaps were generated from the mean values per parameter for each surface, which were scaled to a range from 0-10 based on the highest value per parameter. In order to visualize effects more clearly, scaled data were subtracted from control data to obtain subtraction heatmaps. The effects in subtracted heatmaps were filtered based on significant differences or $1 x$ standard deviation as indicated.

\section{Statistical analysis}

Data are presented as mean \pm SEM. GraphPad Prism 8.3.0 software (La Jolla CA, USA) was used for statistical analyses, using the paired and nonparametric Wilcoxon test to compare means of 2 parallel experimental conditions and one-way non-parametric Anova (Kruskal-Wallis) for experiments with more than 2 conditions. In case of multiple experimental conditions and timepoints, a nonparametric 2-way Anova was used. A p-value less than 0.05 was considered statistically significant in which * is $p<0.05 ; * *$ is $p<0.01$ and $* * *$ is $p<0.001$. 


\section{Results}

\section{Sunitinib reduces thrombus formation and PS exposure in whole blood under flow on collagen type I and III}

To widely explore the multi-target inhibition of sunitinib on thrombus formation, we investigated thrombus formation induced by several TK-dependent receptors using multiple surfaces for coating in whole blood flow chamber experiments. First, we performed doseresponse studies with sunitinib and showed that approximately $3 x$ higher concentration of this compound was required to inhibit aggregation induced by collagen $(1 \mu \mathrm{g} / \mathrm{ml})$ in the presence of plasma when compared to washed platelets (Suppl. Figure 4.1). This agrees with the knowledge that sunitinib is highly protein-bound ${ }^{1}$.We therefore assessed the effects on thrombus formation during whole blood perfusion under flow using $30 \mu \mathrm{M}$ sunitinib. This dose is in range with the plasma concentrations observed in patients with higher peak levels of sunitinib ${ }^{19}$, but exceeded the therapeutic target levels ${ }^{1}$.

The flow studies were performed in a microspot setting, allowing the quasi-simultaneous measurement of sunitinib on adhesive surfaces consisting of collagen type I, collagen type III, VWF co-coated with rhodocytin, VWF co-coated with laminin, VWF co-coated with fibrinogen and VWF co-coated with ristocetin. Hence, roles of different platelet receptors, such as GPVI, CLEC-2, GPIb and integrin $\alpha_{6} \beta_{1}$ and $\alpha_{\| 1} \beta_{3}$, were investigated as described before ${ }^{20-22}$. Captured microscopic images were analysed (Suppl. Figure 4.2), and a heatmap was generated for a more systematic analysis of the seven thrombus parameters, which were averaged and scaled per parameter (Figure 4.1). Parameters of thrombus formation were only consistently affected on GPVIdependent surfaces, i.e. collagen type I and III (Figure 4.1A, B). In the presence of sunitinib, these thrombi were smaller and less compact in structure as compared to the control condition (Suppl. Figure 4.2A and B, brightfield images). Quantification showed a reduction in platelet deposition, thrombus height and contraction (Figure 4.1). P-selectin exposure was significantly reduced in thrombi formed on collagen III and VWF co-coated with laminin, fibrinogen and ristocetin (Suppl. Figure 4.2B and 4.2C). Notably, thrombus parameters for CLEC-2, GPIb- and integrin $\alpha_{116} \beta_{3} / \alpha_{6} \beta_{1}$ dependent surfaces were not affected by sunitinib (Figure 4.1C-F, Suppl. Figure 4.2C-F), suggesting a mostly GPVI-specific effect.

\section{Sunitinib inhibits multiple tyrosine kinases, intracellular $\mathrm{Ca}^{2+}$ responses and PS exposure upon GPVI stimulation}

We have previously shown that overall tyrosine phosphorylation upon GPVI stimulation is inhibited by sunitinib ${ }^{5}$. To study the effect of sunitinib on GPVI-induced phosphorylation of multiple specific TKs simultaneously, we performed a PamGene kinase assay. We observed that in isolated platelets, in total 34 TKs were significantly phosphorylated by stimulation with CRP-XL, which were subsequently significantly inhibited by sunitinib preincubation (Figure 4.2A). Most 
(A)

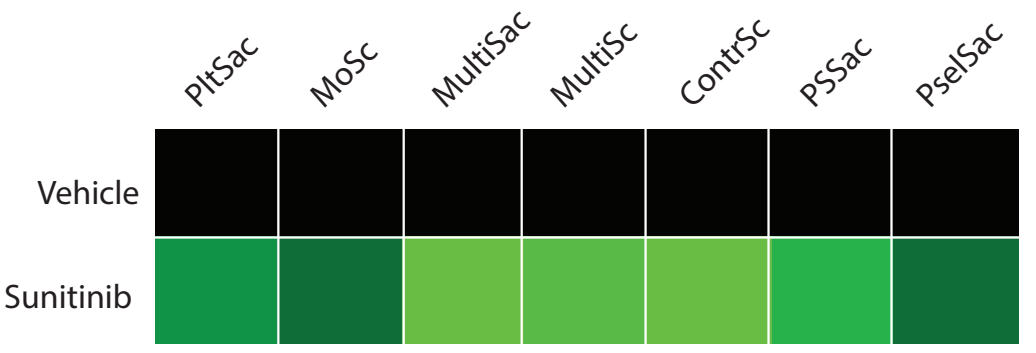

Sunitinib effects

(B)

Vehicle

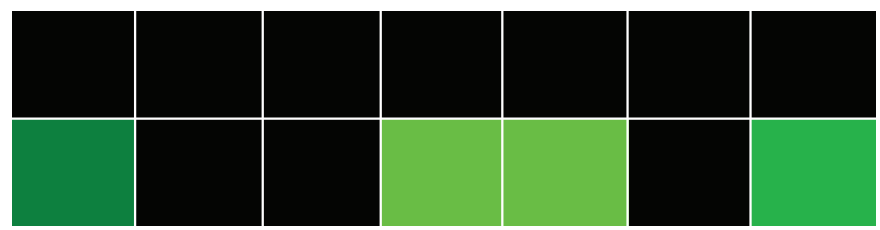

Collagen

type III

Sunitinib

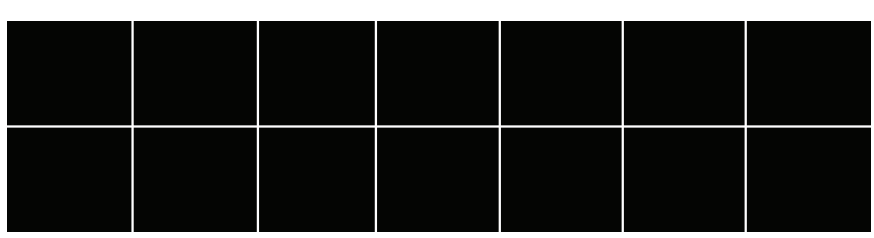

VWF +

(C)

Vehicle

Sunitinib
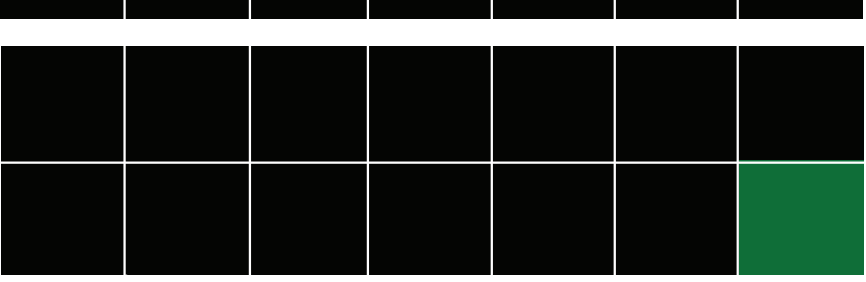

Rhodocytin

(D)

Vehicle

Sunitinib

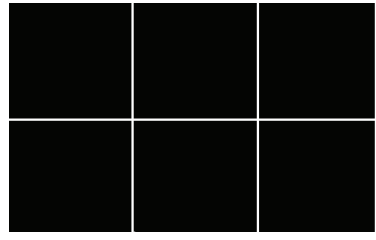

VWF +

Laminin

(E)

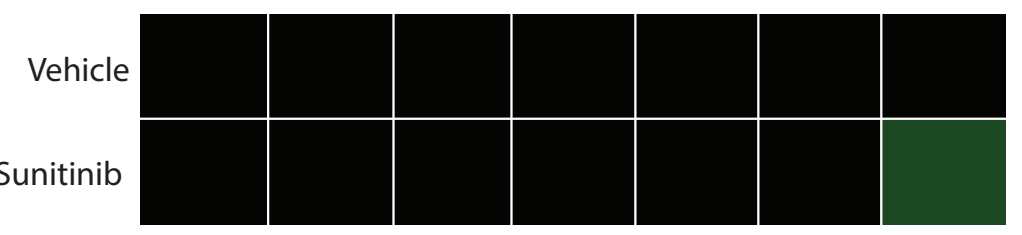

VWF +

Fibrinogen

(F)

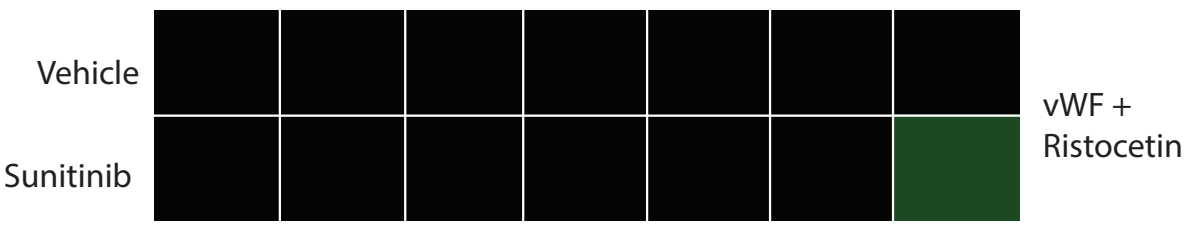

Figure 4.1. Sunitinib strongly reduces thrombus formation on GPVI-dependent surfaces, as compared to surfaces for CLEC-2, GPIb, integrin $\boldsymbol{\alpha}_{6} \boldsymbol{B}_{1}$ and $\boldsymbol{\alpha}_{\| b} \boldsymbol{\beta}_{3}$. Whole blood from healthy volunteers was preincubated with vehicle ( $\left.<0.1 \% \mathrm{DMSO}\right)$ or sunitinib $(30 \mu \mathrm{M})$ for $10 \mathrm{~min}$ and perfused for 3.5 minutes at a wall shear rate of 1,000 $\mathrm{s}^{-1}$ over coated microspots containing (A) collagen type I, (B) collagen type III and (C) VWF plus rhodocytin, (D) VWF plus laminin, (E) vWF plus fibrinogen and (F) VWF plus ristocetin. Subtraction heatmaps are shown representing significant effects of normalized values from the following parameters: platelet deposition (PItSac), morphological score (MoSc), multilayer deposition (MultiSac), multilayer score (MultiSc), contraction score (ContrSc), PS exposure deposition (PSSac) and P-selectin deposition (PselSac). Controls with addition of vehicle (<0.1\% DMSO) were set at 0 for reference. Effects were filtered for significant changes $(p<0.05)$. 
relevant for GPVI signalling are Syk and the SFKs Src, Fyn, and Yes, which all show a high level of inhibition in the presence of sunitinib. Interestingly, the Focal Adhesion Kinases (FAK1 and 2) which signal mostly downstream of integrins ${ }^{23}$, as well as the Gas6 receptor Tyro3/Sky showed a similar pattern of regulation. Furthermore, also other non-GPVI-linked TKs were activated upon GPVI stimulation, most likely via agonists secreted from the $\alpha$-granules. Overall, this assay clearly shows the multi-target nature of sunitinib with regard to TK inhibition in platelets.

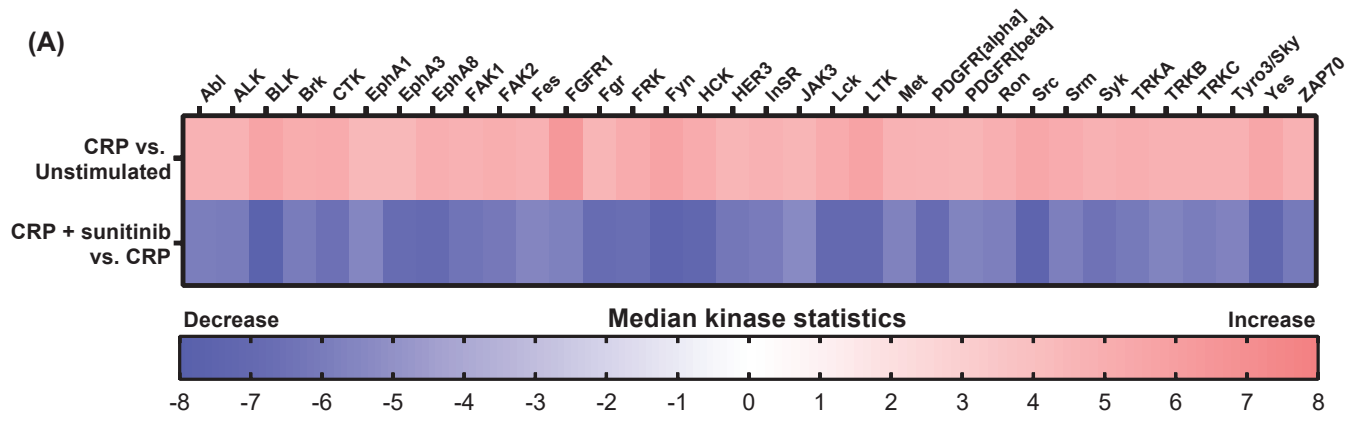

(B)
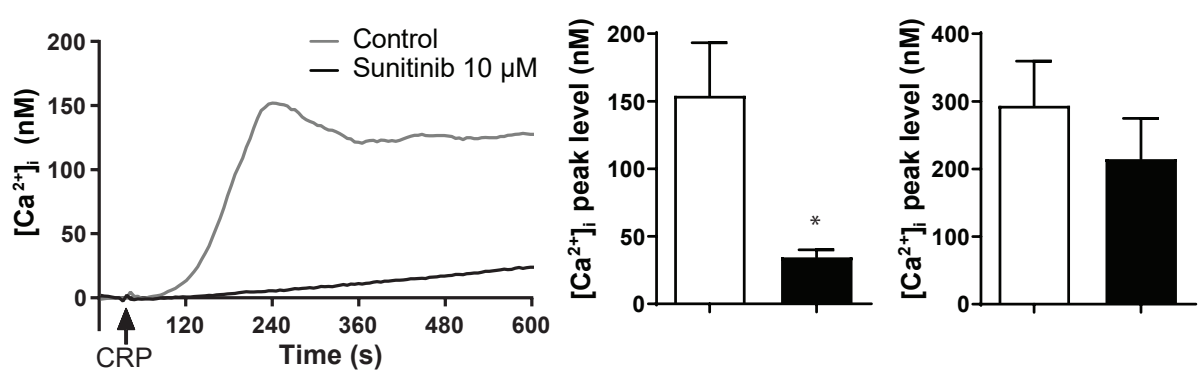

(C)

\section{CRP + Thrombin}

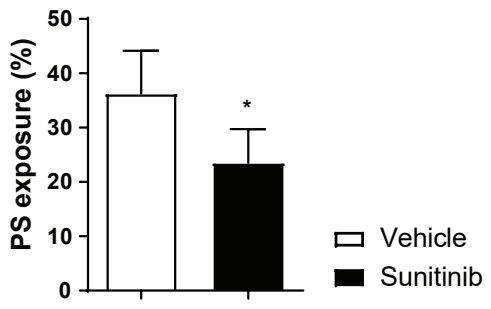

Figure 4.2. Sunitinib inhibits GPVI-induced tyrosine kinase activity, $\mathrm{Ca}^{2+}$ responses and PS exposure in washed platelets. (A) Heatmaps visualize all tyrosine kinases that are significantly up- or downregulated in both comparisons (sorted alphabetically). Cut-off has been set at a median final score of at least 1.2. Colour indicator reflects the calculated median kinase statistic reflecting effect size and directionality (red: upregulated; blue: downregulated). (B) Representative curve of a calcium response of Fura-2 loaded platelets $\left(200 \times 10^{9} / \mathrm{L}\right)$; arrow indicates addition of CRP-XL $(5 \mu \mathrm{g} / \mathrm{mL})$. Bar graphs show quantification of maximum (peak) level of the calcium response induced by CRP-XL ( $5 \mu \mathrm{g} / \mathrm{mL}$ ) or thrombin ( $1 \mathrm{nM}$ ) of Fura-2 loaded platelets in presence (black bars) or absence of sunitinib (white bars). All data are represented as means + SEM ( $n=5-7),{ }^{*} p<0.05$. (C) Isolated platelets from healthy volunteers were incubated with vehicle $(<0.1 \%$ DMSO) or sunitinib $(10 \mu \mathrm{M})$ for 10 minutes at RT. Platelets $\left(100 \times 10^{9} / \mathrm{L}\right)$ were activated in the presence (black bars) or absence of sunitinib (white bars) by CRP-XL $(5 \mu \mathrm{g} / \mathrm{mL})$ in combination with thrombin $(4 \mathrm{nM})$ and incubated for 60 minutes at $37^{\circ} \mathrm{C}$. PS exposure was detected by FITC-conjugated annexin A5 and measured by flow cytometry. 


\section{Chapter 4}

GPVI signalling results in sustained elevation of intracellular calcium levels $\left(\left[\mathrm{Ca}^{2+}\right]_{\mathrm{i}}\right)$ upon stimulation, resulting in PS-exposure ${ }^{24}$. In accordance with the effects on TK phosphorylation, also the $\mathrm{Ca}^{2+}$ increase in response to CRP-XL was nearly abolished in the presence of sunitinib (Figure 4.2B). Elevation in $\left[\mathrm{Ca}^{2+}\right]_{i}$ upon thrombin stimulation was not significantly affected. Also, platelet procoagulant activity (PS exposure), was inhibited by sunitinib in isolated platelets stimulated with CRP plus thrombin (Figure 4.2C). This was in agreement the reduction of PS exposure in whole blood under flow, only on collagen type I (Figure 4.1A).

Considering that GPVI and CLEC-2 display many similarities in the downstream signalling pathways ${ }^{25}$, we additionally determined the effects of sunitinib on CLEC-2 dependent platelet activation. We observed that in isolated platelets, both rhodocytin-induced aggregation, as well as activation of integrin $\alpha_{\| 1} \beta_{3}$ and expression of P-selectin were significantly reduced by sunitinib (Suppl. Fig 4.3A, B). Also, PS exposure as induced by rhodocytin plus thrombin was significantly reduced by sunitinib (Suppl. Fig. 4.3C). However, we did not observe an effect of sunitinib in whole blood thrombus formation on rhodocytin plus vWF (Fig. 4.1C, Suppl. Fig 4.2C), suggesting that CLEC-2-induced thrombus formation was not affected by sunitinib in whole blood.

So far, all data showed that sunitinib most prominently reduced phosphorylation of TKs and rises of intracellular $\mathrm{Ca}^{2+}$ levels, resulting in inhibition of thrombus formation and PS exposure induced by the collagen receptor GPVI. Therefore, we used collagen type I for further experiments.

\section{Aspirin enhances sunitinib-induced inhibition of platelet aggregation and thrombus formation}

To investigate the combined effect of sunitinib and aspirin on platelet function, we started by measuring platelet aggregation. Sunitinib alone caused a minor inhibition $( \pm 14 \%)$ of the aggregation response induced by a high dose $(5 \mu \mathrm{g} / \mathrm{ml})$ of collagen, whereas aspirin inhibited this collagen-induced aggregation by $\pm 70 \%$ (Figure $4.3 \mathrm{~A}$ ). The combination of sunitinib and aspirin reduced the aggregation response even further as compared to aspirin alone (>80\%; Figure 4.3A). Similar results could be observed when platelets were stimulated with 2MeS-ADP (Figure 4.3B). However, both sunitinib and/or aspirin treatment did not affect the aggregation response elicited by thrombin (Figure 4.3C).

Next, we determined the effects of sunitinib and/or aspirin in whole blood thrombus formation over collagen type I. When aspirin was added in vitro, we only observed a significant effect on thrombus contraction, while other parameters were not affected (Figure 4.3D). No additional effects on thrombus formation in whole blood could be observed when both aspirin and sunitinib were added in vitro (Figure 4.3D). Subsequently, we obtained blood from healthy volunteers who had taken a single dose of aspirin (100 mg) one day before. Treatment with aspirin inhibited platelet deposition as compared to controls without aspirin intake (Figure 4.3E, Suppl. Figure 4.4). Addition of sunitinib to blood from aspirin-treated donors further enhanced the 
effects that were observed with aspirin alone (Figure 4.3E). Especially thrombus height (multilayer) and PS exposure were reduced when both drugs were present, as compared to aspirin alone (Figure 4.3E). Altogether, these results suggest that dual treatment with anti-platelet therapy and sunitinib increases the inhibition of platelet function.

(A)

Collagen

(B)

ADP

(C) Thrombin
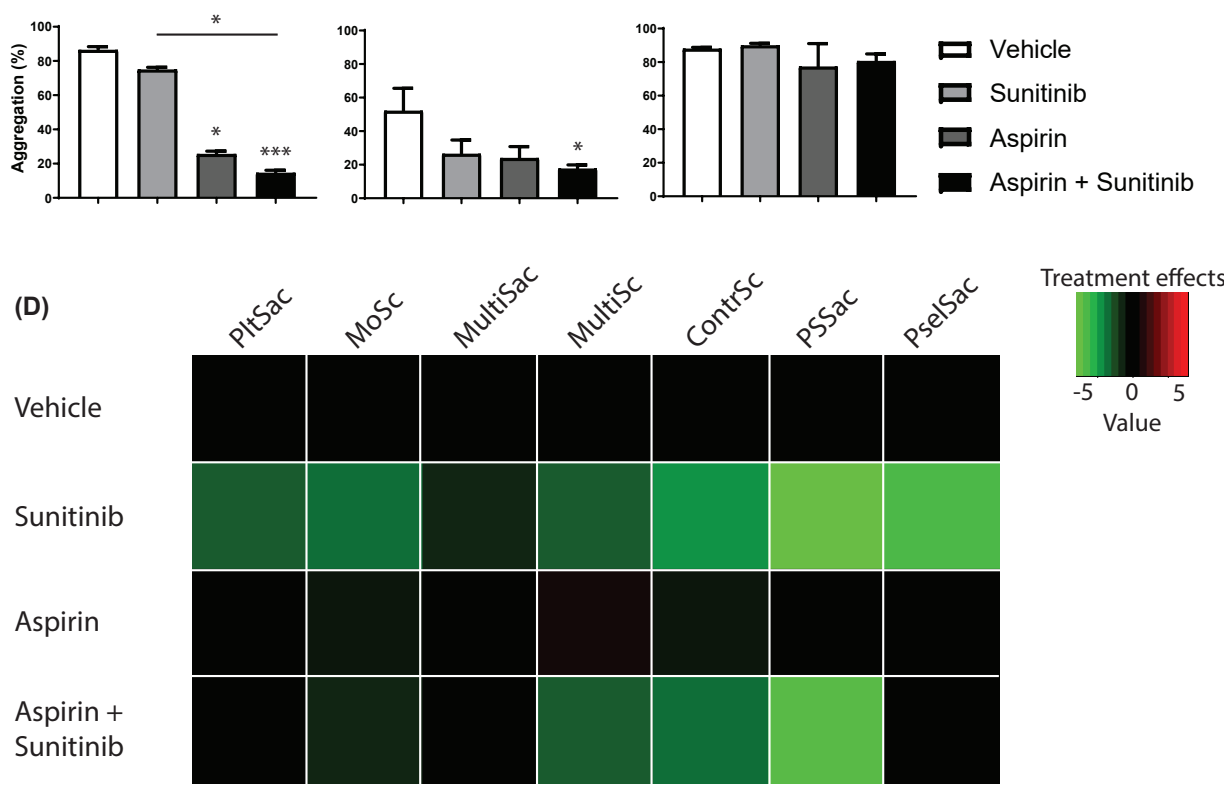

(E)

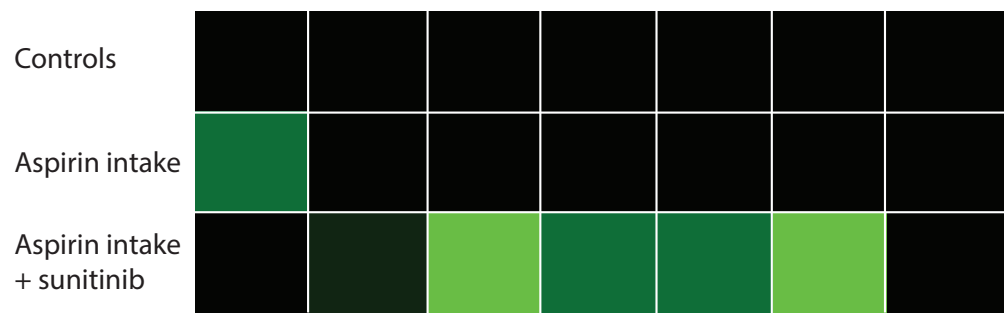

Figure 4.3. Aspirin enhances the effect of sunitinib in collagen-induced platelet aggregation and in ex vivo thrombus formation on collagen in whole blood after aspirin intake. Washed platelets $\left(250 \times 10^{9} / \mathrm{L}\right)$ were incubated with vehicle $(<0.1 \% \mathrm{DMSO})$, aspirin $(100 \mu \mathrm{M})$ and/or sunitinib $(10 \mu \mathrm{M})$. Aggregation of washed platelets was induced by $(\mathrm{A}) 5 \mu \mathrm{g} / \mathrm{mL}$ collagen, (B) $1 \mu \mathrm{M} 2 \mathrm{MeS}-\mathrm{ADP}$ or (C) $1 \mathrm{nM}$ thrombin. Data are represented as means + SEM (n=3), * $\mathrm{p}<0.05, * * * \mathrm{p}<0.001$. $(\mathrm{D}, \mathrm{E})$ Whole blood from healthy volunteers was preincubated with vehicle $(<0.1 \% \mathrm{DMSO})$, sunitinib $(30 \mu \mathrm{M})$ and/or aspirin $(100 \mu \mathrm{M})$ for $10 \mathrm{~min}$. Recalcified whole blood was perfused over a collagen surface at $1000 \mathrm{~s}^{-1}$ for 3.5 minutes, followed by perfusion with buffer supplemented with AF568-annexin A5 and AF647- $\alpha$-CD62P to detect PS exposure and P-selectin expression, respectively. (D) Subtraction heatmaps representing the significant effects of normalized values of the parameters. Controls with addition of vehicle $(<0.1 \% \mathrm{DMSO})$ were set at 0 for reference. Effects were filtered for significant alterations by addition of sunitinib and/or aspirin. (E) Subtraction heatmaps representing the significant effects of normalized values of parameters as for supplemental Figure 4.4B. Healthy controls were set at 0 for reference to aspirin intake or aspirin intake with in vitro addition of sunitinib. Effects were filtered for significant changes of the different platelet parameters $(\mathrm{p} \leq 0.05)$. 
(A)
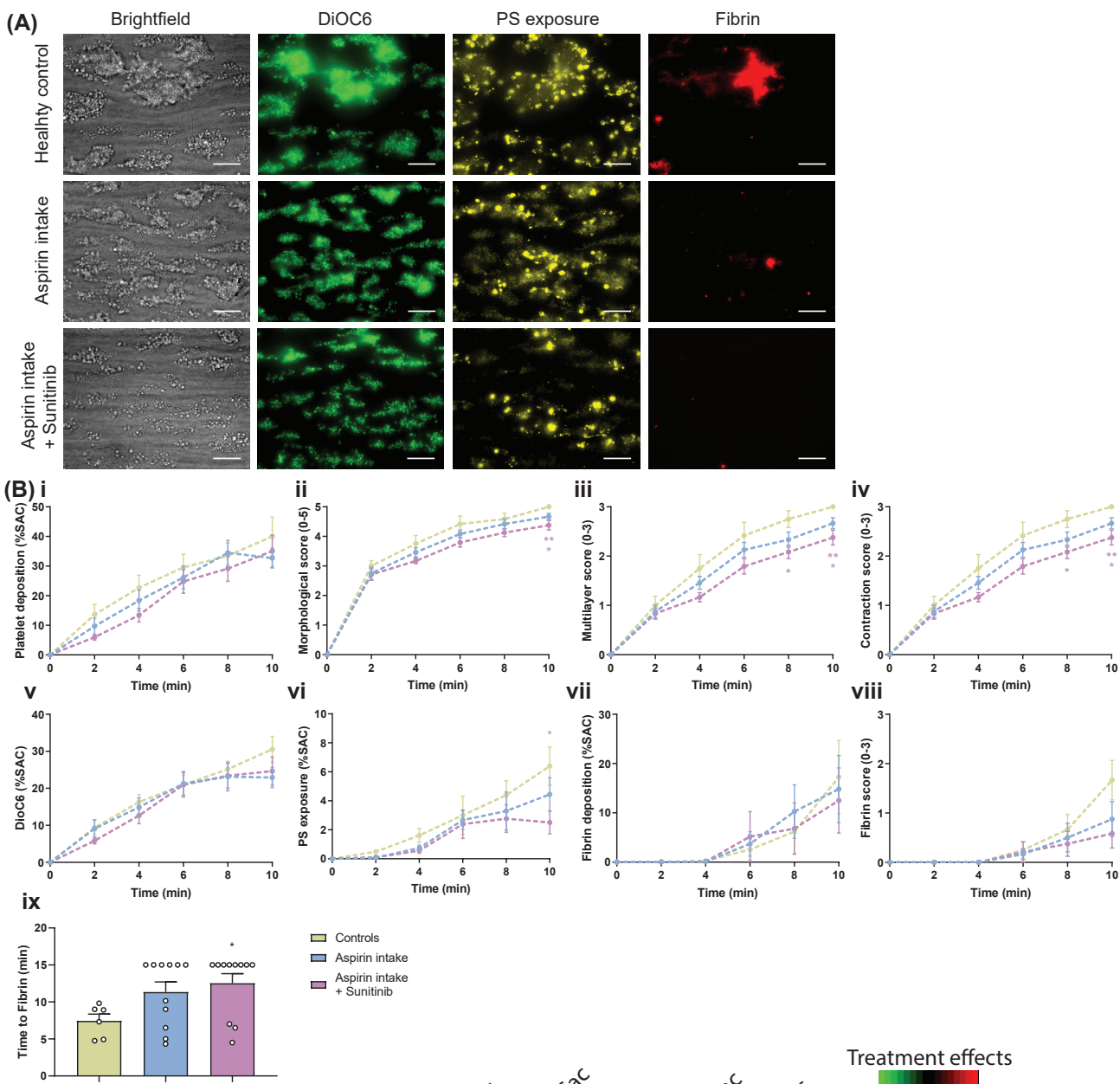

$$
\begin{aligned}
& \square \text { Controls } \\
& \square \text { Aspirin intake } \\
& \square \begin{array}{l}
\text { Aspirin intake } \\
+ \text { Sunitinib }
\end{array}
\end{aligned}
$$

(C)

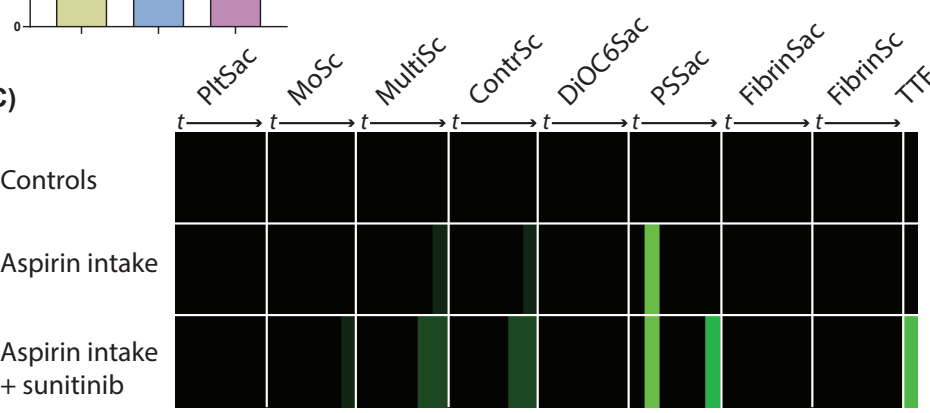

Treatment effects

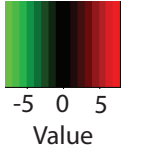

Figure 4.4. Aspirin partially enhances the inhibiting effect of sunitinib in platelet-dependent fibrin formation under flow. Citrated whole blood of healthy volunteers with single dose of aspirin was treated in vitro with vehicle $(<0.1 \%$ DMSO) or sunitinib $(30 \mu \mathrm{M})$ for $10 \mathrm{~min}$ followed by addition of DiOC6 (platelet deposition), AF568-annexin A5 (PSexposure) and AF647- $\alpha$-fibrinogen (fibrin). Blood was co-perfused with $\mathrm{CaCl}_{2} / \mathrm{MgCl}_{2}$ over collagen type I at a wall shear rate of $1000 \mathrm{~s}^{-1}$. (A) Representative brightfield and fluorescence images taken after 10 minutes of blood perfusion. (B) Kinetic analysis of brightfield images of (i) platelet deposition (PItSac), (ii) morphological score (MoSc), (iii) multilayer score (MultiSc), and (iv) contraction score (ContrSc) and of fluorescence images of (v) DiOC6 platelet deposition (DiOC6Sac), (vi) PS exposure (PSSac), (vii) fibrin deposition (FibrinSac), (viii) fibrin score (FibrinSc) and (ix) time to fibrin (TTF). Data are represented as means + SEM $(n=6),{ }^{*} p<0.05,{ }^{* *} p<0.01$. (C) Heatmap representing the significant effects of normalized values of parameters analysed in $\mathrm{B}$. Healthy controls were set at 0 for reference to aspirin intake or aspirin intake with in vitro addition of sunitinib. Effects were filtered for significant changes of the different platelet parameters $(p \leq 0.05)$. 


\section{Aspirin enhances sunitinib effects on thrombus fibrin formation}

As we observed that the exposure of procoagulant PS was reduced by sunitinib (plus aspirin), we further investigated whether these effects extended to conditions of thrombin generation and fibrin formation. Hence, we assessed the kinetics of platelet-fibrin thrombus formation under flow on a collagen surface co-coated with $\mathrm{TF}^{18}$. Recalcified whole blood was labelled with DiOC6, AF568-Annexin A5 and AF647-fibrinogen, and was perfused over a microspot consisting of collagen type I plus TF. Using control blood samples, we observed timedependent increases in platelet deposition and thrombus formation in time, with the start of fibrin formation after 7-8 minutes (Figure 4.4A). Preincubation of blood with sunitinib did not affect the parameters of platelet deposition and thrombus size in time, but sunitinib significantly reduced PS exposure with a concomitant delay in fibrin formation (Suppl. Figure 4.5). Of note, sunitinib did not affect thrombin generation under static conditions, as measured with the calibrated automatic thrombogram ${ }^{26}$ in PRP stimulated with collagen (data not shown). Markedly, using blood from aspirin-treated healthy volunteers, we observed a reduction in thrombus size, height and density as compared to healthy volunteers without aspirin intake (Figure 4.4A, B). Furthermore, a slight decrease in PS exposure as well as a delay in fibrin formation was observed upon aspirin intake (Figure 4.4). These effects were further reduced by in vitro addition of sunitinib (Figure 4.4C). Especially, effects on thrombus height, contraction, PS exposure and time to fibrin generation were increased upon dual treatment with aspirin and sunitinib as compared to aspirin alone. Together, these results suggest that sunitinib reduces GPVI-induced activation of platelets resulting in decreased PS exposure and thereby delaying the time for fibrin to be formed, which is enhanced by aspirin intake.

\section{Sunitinib reduces platelet-dependent fibrin formation under flow in whole blood from cancer patients}

Previously, we demonstrated that sunitinib reduced thrombus formation and PS exposure in whole blood from renal cell carcinoma (RCC) patients under non-coagulating conditions 5 . To explore whether sunitinib could also reduce platelet-dependent fibrin formation under flow in blood from cancer patients, we obtained blood from 11 patients diagnosed with lung, pancreatic and (neuro)glioblastoma cancer (Suppl. Table 4.1). Sunitinib has been reported to be used as a (combination) therapeutic strategy for these types of cancer ${ }^{27-29}$. As a proof-of-principle, we preincubated recalcified whole blood from these patients with vehicle or sunitinib followed by labelling with DiOC6, AF568-Annexin A5 and AF647-fibrinogen. Upon perfusion of the vehicletreated blood over collagen co-coated with TF, we observed increases in platelet deposition and thrombus formation in time, with a median start of fibrin formation after 7-8 minutes, comparable to healthy controls (Suppl. Figure 4.6A, B). Three patients were treated with anticoagulant or antiplatelet drugs (Suppl. Table 4.1), but this did not affect thrombus/fibrin formation as 
(A) $\mathrm{i}$

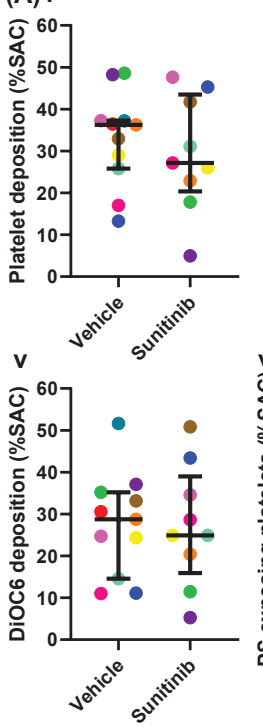

ii
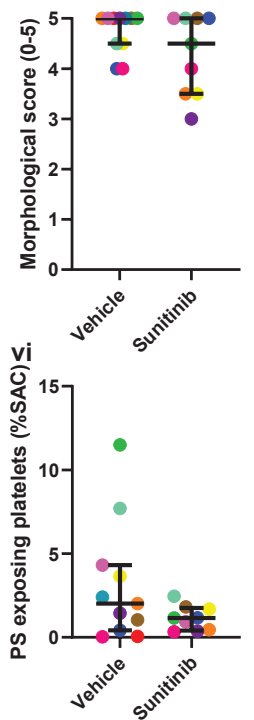

iii
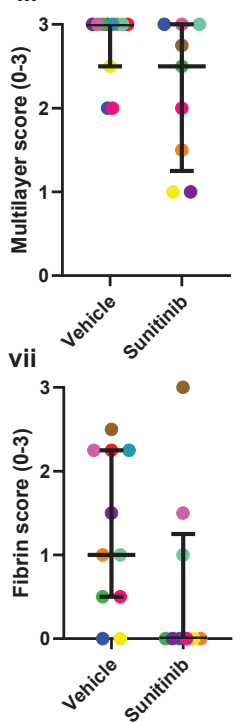

iv
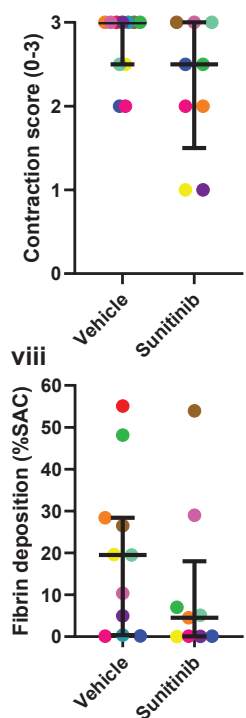

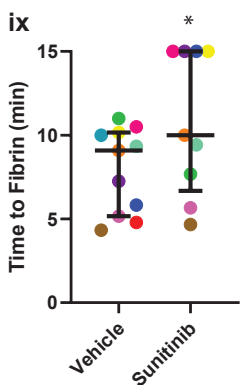

(B)

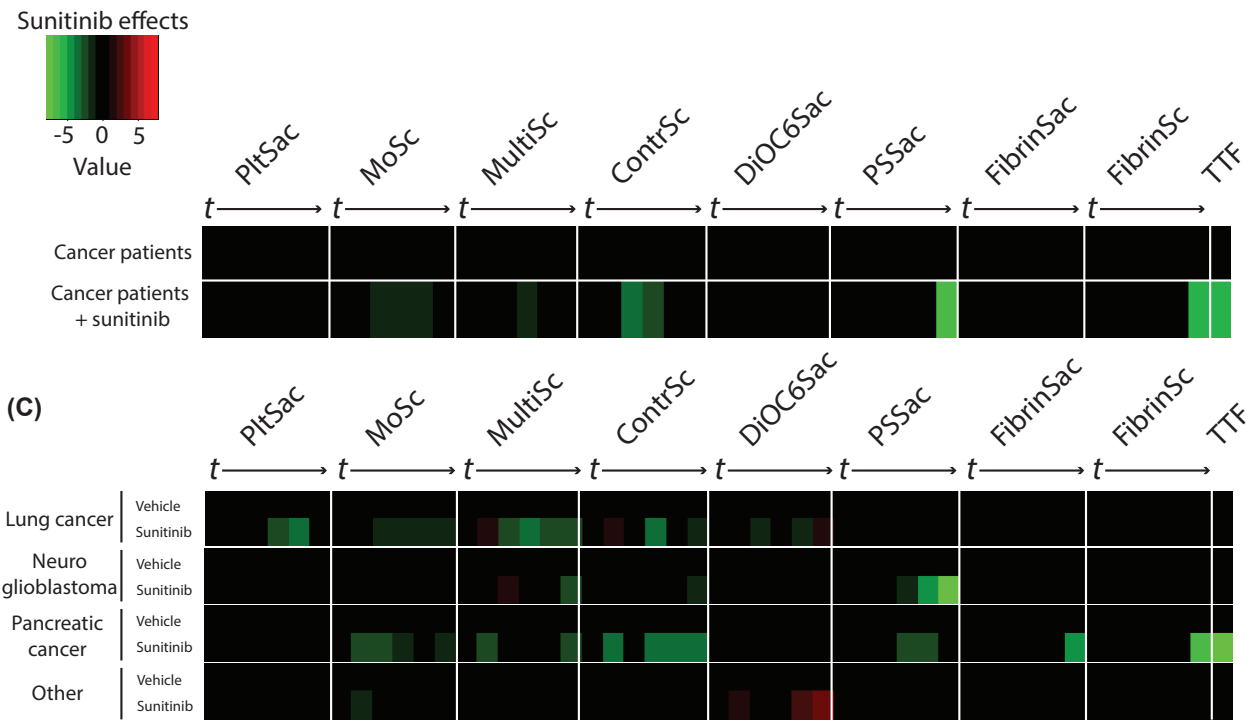

Figure 4.5. Sunitinib reduces platelet-dependent fibrin formation under flow in whole blood from cancer patients. Citrated whole blood from cancer patients (suppl. Table I) was treated in vitro with vehicle $(<0.1 \%$ DMSO) or sunitinib $(30 \mu \mathrm{M})$ for $10 \mathrm{~min}$, followed by addition of DiOC6 (platelet deposition), AF568-annexin A5 (PS-exposure) and AF647$\alpha$-fibrinogen (fibrin). Blood was co-perfused with $\mathrm{CaCl}_{2} / \mathrm{MgCl}_{2}$ over collagen type I plus TF at a wall shear rate of 1000 $\mathrm{s}^{-1}$. (A) Endpoint analysis of brightfield images of (i) platelet deposition (PltSac), (ii) morphological score (MoSc), (iii) multilayer score (MultiSc), and (iv) contraction score (ContrSc) and of fluorescence images of (v) DiOC6 platelet deposition (DiOC6Sac), (vi) PS exposure (PSSac), (vii) fibrin deposition (FibrinSac), (viii) fibrin score (FibrinSc) and (ix) time to fibrin (TTF). Data are represented as median \pm interquartile range $(n=9-11)$. Each dot represents an individual cancer patient (colour-coding in Suppl. Table 4.1), ${ }^{*} p<0.05$. (B) Subtraction heatmap representing the overall effect of sunitinib in cancer patients. The effects of normalized values of parameters during time are based on the analysis of Suppl. Figure 7. Vehicle treated blood of cancer patients was set at 0 for reference to in vitro addition of sunitinib. Effects were filtered for significant changes between the different platelet parameters $(p \leq 0.05)$. (C) Subtraction heatmap of the effect of sunitinib per cancer type. Effects were filtered for changes greater than the 1x SD range of the different platelet parameters. 
compared to the patients without this treatment. Preincubation of blood with sunitinib showed a trend in overall inhibition of thrombus size and height in time, as well as a reduction in PS exposure with a significant delay in fibrin formation (Figure 4.5A and Suppl. Figure 4.6A, B). To visualize the overall effects of sunitinib in blood from cancer patients, we generated a heatmap (Figure 4.5B). This heatmap clearly shows that sunitinib inhibits platelet PS-exposure and fibrin formation, but also some effects on thrombus formation parameters can be observed. We included patients with different types of cancer, which this may result in different cancer-specific responses. Therefore, we also generated a heatmap with the patients averaged per cancer type (Figure 4.5C). This heatmap shows that in lung and pancreatic cancer, more pronounced effects of sunitinib were observed on thrombus formation parameters (i.e. height, size and contraction) as compared to neuroglioblastoma and other cancer types. This variable response to sunitinib treatment was even more pronounced when a heatmap was generated per patient (suppl. Figure 4.6C). This heatmap shows that the effects of sunitinib on thrombus and fibrin formation under flow varied more between individual patients than between the types of cancer. Overall, in 6 of the 9 patients were sunitinib was added we observed clear inhibition of thrombus formation and/or PS exposure and/or fibrin generation. This suggested that the in vitro inhibitory effects of sunitinib were more pronounced in cancer patients (Figure 4.5C and suppl. Figure 4.6C) than in healthy controls (Suppl. Figure 4.5).

\section{Discussion}

In the present study we further explored the multi-target inhibition of sunitinib on thrombus formation, by investigating thrombus formation by stimulation with multiple agonists, as well as under coagulating conditions stimulated by collagen plus TF. We observed that sunitinib predominantly inhibited collagen-induced exposure of phosphatidylserine, resulting in delayed fibrin formation. Furthermore, we found an enhancing effect of dual treatment of platelets with sunitinib and aspirin in these processes. Finally, platelet-dependent fibrin formation in whole blood from cancer patients was significantly inhibited by sunitinib.

We have previously reported that platelets sequester sunitinib in vitro as well as in cancer patients and that this uptake inhibits platelet function in response to collagen ${ }^{5}$. As sunitinib is a multi-target TKI and TKs do not only signal under the collagen receptor GPVI, but also under the receptors GPIb, CLEC-2 and integrins $\alpha_{6} \beta_{1}$ and $\alpha_{11 \mathrm{~b}} \beta_{3}{ }^{21}$, we investigated the effects of sunitinib on thrombus formation in whole blood perfused over multiple agonists. However, sunitinib did not affect thrombus formation on CLEC-2, GPIb- and integrin $\alpha_{1 \mathrm{lb}} \beta_{3} / \alpha_{6} \beta_{1}$-dependent surfaces, which pointed to GPVI as the main signalling pathway inhibited by this TKI. We did observe that sunitinib inhibited CLEC-2-induced aggregation and activation responses in isolated platelets. This is in line with the many similarities in the downstream signalling pathways underneath GPVI and CLEC-2 ${ }^{25}$. The inhibiting effects of sunitinib on CLEC-2-induced thrombus formation were however absent in 


\section{Chapter 4}

whole blood. This may be explained by a stronger dependence on Syk of CLEC-2 signalling, while GPVI signalling is more dependent on $\mathrm{Src}^{25}$. Inhibition of Src by sunitinib upon GPVI stimulation was more pronounced as compared to inhibition of Syk (Figure 4.2A), suggesting that the effects of sunitinib on CLEC-2 signalling may be less prominent, especially in whole blood conditions. Furthermore, we noted that higher collagen doses overrule the inhibiting effects of sunitinib at $10 \mu \mathrm{M}$. Hence, a higher concentration of sunitinib may be required to inhibit platelet activation at higher agonist concentrations. A possible role for integrin $\alpha_{2} \beta_{1}$, the other collagen receptor on platelets, should be mentioned here as well, as this receptor also signals via Src and Syk kinases ${ }^{30}$. It has been shown that integrin $\alpha_{2} \beta_{1}$ has a supporting role with regard to platelet activation and contributes to stable thrombus formation on collagen ${ }^{31}$. As we have shown that sunitinib strongly reduced phosphorylation of Syk and Src, the inhibiting effects observed in thrombus formation on collagen may be partly due to inhibition of integrin $\alpha_{2} \beta_{1}$ signalling. In addition, thrombus formation under flow on collagen type III was reduced by sunitinib treatment, although not as strong as compared to thrombus formation on collagen type I. Together, this is consistent with the multi-target nature of sunitinib as a broad-spectrum protein TKI, having lower affinity for multiple TKs rather than high affinity for one specific signalling protein ${ }^{32,33}$. This also explains why the effects of sunitinib on platelet activation are not as strong as compared to specific inhibitors of Syk or $\mathrm{Src}^{34,35}$.

We showed that in total 34 TKs were activated upon GPVI stimulation, which were subsequently inhibited in the presence of sunitinib. Several TKs could be directly linked to GPVI signalling, such as Syk and the SFKs, but most TKs were most likely activated by agonists secreted from the $\alpha$-granules upon GPVI stimulation, such as fibrinogen, activating FAK1/2 via integrin $\alpha_{111} \beta_{3}{ }^{23}$, as well as Gas6, activating Tyro3/Sky. As platelets contain many bioactive molecules in their granules, most likely also the other non-ITAM-linked TKs that showed phosphorylation upon GPVI stimulation, have been activated via similar secondary events. As we have shown that platelet secretion is inhibited by sunitinib ${ }^{5}$, the inhibiting effects of sunitinib on non-GPVI-linked TKs may also be due to reduced secretion of secondary mediators. Prolonged elevation of intracellular calcium levels is a prerequisite for platelet PS exposure, a hallmark of procoagulant activity ${ }^{24}$. The observed effects of sunitinib on intracellular calcium rises in response to GPVI stimulation fits with the currently observed reduction in TK phosphorylation and agrees with previously shown effects on collagen-induced tyrosine phosphorylation of multiple proteins ${ }^{5}$. This implies interference of sunitinib early in the signalling cascade used by the collagen receptor GPVI, e.g. with TKs in the GPVI signalosome or with Syk as a central regulator of collagen-induced platelet activation. In agreement, another study reported that collagen-induced tyrosine phosphorylation of c-Src is reduced in platelets treated with sunitinib ${ }^{6}$. Others have shown that also in cardiomyocytes sunitinib treatment results in reduced calcium responses accompanied with decreased sarcomere shortening ${ }^{36}$. We showed that sunitinib reduced platelet PS exposure in isolated platelets in vitro, 
as well as during whole blood thrombus formation under flow in the presence and absence of coagulation. This reduction in platelet procoagulant activity upon sunitinib treatment resulted in delayed and reduced fibrin clot formation. An earlier study with other TKIs has also demonstrated that platelet tyrosine phosphorylation is required for procoagulant activity and fibrin formation ${ }^{37}$.

As both aspirin and sunitinib are associated with increased bleeding risk ${ }^{3,9}$, we investigated if the combination of these drugs could reinforce the inhibiting effects on platelets. We observed that indeed this aspirin-sunitinib combination resulted in stronger inhibition of collagen-induced platelet aggregation as compared to either compound alone. However, in whole blood perfusion experiments (with and without coagulation) we did not observe these additive effects when aspirin and sunitinib were added in vitro. In contrast, platelet deposition and thrombus height were reduced in blood from aspirin-treated donors as compared to controls without aspirin intake. Moreover, addition of sunitinib further enhanced the effects that were observed with aspirin alone, accompanied by further reductions in PS exposure and fibrin formation. In an earlier study from our group with patients suffering from peripheral artery disease (PAD), no effects of aspirin intake were observed on thrombus formation under flow as compared to healthy controls without aspirin intake ${ }^{38}$. This could be due to the increased platelet reactivity observed in these PAD patients, that was counterbalanced by the aspirin intake. In contrast, in this study in vitro addition of aspirin to blood from healthy volunteers did reduce thrombus formation and PS exposure under flow ${ }^{38}$, which was also observed by others ${ }^{39}, 40$. The present findings are compatible to the variable effects of aspirin intake alone on thrombus formation, while the combination of aspirin with other anti-platelet drugs causes a marked reduction ${ }^{41-45}$. Furthermore, a recent study has shown concomitant effects of aspirin and ibrutinib on collageninduced aggregation ${ }^{46}$. Altogether, these results suggest that dual treatment with anti-platelet therapy and TKIs increase the inhibition of platelet function.

In the present study we included a limited number of cancer patients, who suffered from different cancer types. Also, we added the sunitinib in vitro, which may be a limitation to the current study. The effects of sunitinib on platelet-dependent coagulation in blood from patients treated with sunitinib requires further study. Also, the combined effects of sunitinib and aspirin or other anti-platelet drugs on platelets from patients on treatment in relation to bleeding should be further investigated. Despite these limitations, we clearly observed in this proof-of-principle study that the in vitro inhibitory effects of sunitinib on thrombus size and height under coagulating conditions were more pronounced in several individual cancer patients as compared to healthy controls. It is known that cancer patients have a procoagulant phenotype ${ }^{47,48}$, and therefore platelets from cancer patients may be more sensitive to inhibitory compounds as compared to platelets from healthy individuals. We also observed more pronounced effects of sunitinib on thrombus formation parameters in lung and pancreatic cancer as compared to neuroglioblastoma and other cancer types. It has been reported that tumour-induced changes in platelet proteins 
and mRNA are tumour-type specific ${ }^{49,50}$, hence we hypothesize that these specific tumourinduced changes may influence the platelet response to inhibiting drugs.

Altogether, we conclude that sunitinib inhibited GPVI-induced phosphorylation of TKs (a.o. Syk and $\mathrm{Src}$ ), with subsequent reduction of $\mathrm{Ca}^{2+}$ elevation, resulting in reduced platelet activation and PS-exposure. This was accompanied by delayed and reduced formation of fibrin, all of which were aggravated in the presence of aspirin. This asks for awareness among clinicians for the combined antiplatelet effects of TKIs together with anti-platelet drugs.

\section{Acknowledgements}

This study was supported by Pfizer as an Investigator-Initiated Research grant to MJEK (Tracking Number WI209458) and funds from the Department of Medicine, University of Padua Medical School (to PS). DIF received funding from the European Union's Horizon 2020 research and innovation program under Marie Sklodowska-Curie grant agreement No. 766118 and is enrolled in a joint PhD program of the universities of Maastricht and Santiago de Compostela. JAE was funded by the Deutsche Forschungsgemeinschaft (DFG grant: SFP1009 project A09). EPCVdV was funded by a grant from the Interdisciplinary Center for Clinical Research within the faculty of Medicine at the RWTH Aachen University and NWO-ZonMw Veni (91619053).

\section{Conflict of Interest Statement}

JWMH is a cofounder and shareholder of FlowChamber B.V. The other authors declare no relevant conflicts of interest.

\section{References}

1. Goodman VL, Rock EP, Dagher R, et al. Approval summary: sunitinib for the treatment of imatinib refractory or intolerant gastrointestinal stromal tumors and advanced renal cell carcinoma. Clin Cancer Res 2007; 13(5): 1367-73.

2. Adams VR, Leggas M. Sunitinib malate for the treatment of metastatic renal cell carcinoma and gastrointestinal stromal tumors. Clin Ther 2007; 29(7): 1338-53.

3. Je Y, Schutz FA, Choueiri TK. Risk of bleeding with vascular endothelial growth factor receptor tyrosine-kinase inhibitors sunitinib and sorafenib: a systematic review and meta-analysis of clinical trials. Lancet Oncol 2009; 10(10): 967-74.

4. Versteeg HH, Heemskerk JW, Levi M, et al. New fundamentals in hemostasis. Physiol Rev 2013; 93(1): 327-58.

5. Sabrkhany S, Griffioen AW, Pineda S, et al. Sunitinib uptake inhibits platelet function in cancer patients. Eur J Cancer 2016; 66: 47-54.

6. Walraven M, Homs MYV, van der Veldt AAM, et al. Platelet function is disturbed by the angiogenesis inhibitors sunitinib and sorafenib, but unaffected by bevacizumab. Angiogenesis 2018; 21(2): 32534.

7. De Stefano V. Arterial thrombosis and cancer: the neglected side of the coin of Trousseau syndrome. Haematologica 2018; 103(9): 1419-21.

8. Navi BB, Reiner AS, Kamel H, et al. Risk of Arterial Thromboembolism in Patients With Cancer. J Am Coll Cardiol 2017; 70(8): 926-38.

9. Silvain J, Cayla G, O'Connor SA, et al. Antiplatelet options for secondary prevention in acute coronary syndromes. Expert Rev Cardiovasc Ther 2011; 9(11): 1403-15. 
10. Arthur JF, Jandeleit-Dahm K, Andrews RK. Platelet Hyperreactivity in Diabetes: Focus on GPVI Signaling-Are Useful Drugs Already Available? Diabetes 2017; 66(1): 7-13.

11. Gresele P, Momi S, Malvestiti M, et al. Platelet-targeted pharmacologic treatments as anti-cancer therapy. Cancer Metastasis Rev 2017; 36(2): 331-55.

12. Haemmerle M, Stone RL, Menter DG, et al. The Platelet Lifeline to Cancer: Challenges and Opportunities. Cancer Cell 2018; 33(6): 965-83.

13. van der Meijden PE, Feijge MA, Giesen PL, et al. Platelet P2Y12 receptors enhance signalling towards procoagulant activity and thrombin generation. A study with healthy subjects and patients at thrombotic risk. Thromb Haemost 2005; 93(6): 1128-36.

14. Feijge MA, van Pampus EC, Lacabaratz-Porret C, et al. Inter-individual variability in $\mathrm{Ca} 2+$ signalling in platelets from healthy volunteers: effects of aspirin and relationship with expression of endomembrane Ca2+-ATPases. Br J Haematol 1998; 102(3): 850-9.

15. Grynkiewicz G, Poenie M, Tsien RY. A new generation of Ca2+ indicators with greatly improved fluorescence properties. J Biol Chem 1985; 260(6): 3440-50.

16. Chirumamilla CS, Fazil M, Perez-Novo C, et al. Profiling Activity of Cellular Kinases in Migrating T-Cells. Methods Mol Biol 2019; 1930: 99-113.

17. de Witt SM, F. S, J. C, et al. Multi-parameter assessment of thrombus formation on microspotted arrays of thrombogenic surfaces. Nat Prot Exchange 2014.

18. Swieringa F, Baaten CC, Verdoold R, et al. Platelet Control of Fibrin Distribution and Microelasticity in Thrombus Formation Under Flow. Arterioscler Thromb Vasc Biol 2016; 36(4): 692-9.

19. Numakura K, Fujiyama N, Takahashi M, et al. Clinical implications of pharmacokinetics of sunitinib malate and $\mathrm{N}$-desethyl-sunitinib plasma concentrations for treatment outcome in metastatic renal cell carcinoma patients. Oncotarget 2018; 9(38): 25277-84.

20. Swieringa F, Kuijpers MJ, Heemskerk JW, et al. Targeting platelet receptor function in thrombus formation: the risk of bleeding. Blood Rev 2014; 28(1): 9-21.

21. Tullemans BME, Heemskerk JWM, Kuijpers MJE. Acquired platelet antagonism: off-target antiplatelet effects of malignancy treatment with tyrosine kinase inhibitors. J Thromb Haemost 2018; 16(9): 1686-99.

22. de Witt SM, Swieringa F, Cavill R, et al. Identification of platelet function defects by multi-parameter assessment of thrombus formation. Nat Commun 2014; 5: 4257.

23. Guidetti GF, Torti M, Canobbio I. Focal Adhesion Kinases in Platelet Function and Thrombosis. Arterioscler Thromb Vasc Biol 2019; 39(5): 857-68.

24. Fernandez DI, Kuijpers MJE, Heemskerk JWM. Platelet calcium signaling by G-protein coupled and ITAM-linked receptors regulating anoctamin-6 and procoagulant activity. Platelets 2020: 1-9.

25. Rayes J, Watson SP, Nieswandt B. Functional significance of the platelet immune receptors GPVI and CLEC-2. J Clin Invest 2019; 129(1): 12-23.

26. Hemker HC, Giesen P, AlDieri R, et al. The calibrated automated thrombogram (CAT): a universal routine test for hyper- and hypocoagulability. Pathophysiol Haemost Thromb 2002; 32(5-6): 249-53.

27. Qiang $\mathrm{H}$, Chang $\mathrm{Q}, \mathrm{Xu}$ J, et al. New advances in antiangiogenic combination therapeutic strategies for advanced non-small cell lung cancer. J Cancer Res Clin Oncol 2020; 146(3): 631-45.

28. Faivre S, Niccoli P, Castellano D, et al. Sunitinib in pancreatic neuroendocrine tumors: updated progression-free survival and final overall survival from a phase III randomized study. Ann Oncol 2017; 28(2): 339-43.

29. Grisanti S, Ferrari VD, Buglione M, et al. Second line treatment of recurrent glioblastoma with sunitinib: results of a phase II study and systematic review of literature. J Neurosurg Sci 2019; 63(4): 458-67.

30. Inoue O, Suzuki-Inoue K, Dean WL, et al. Integrin alpha2beta1 mediates outside-in regulation of platelet spreading on collagen through activation of Src kinases and PLCgamma2. J Cell Biol 2003; 160(5): 769-80.

31. Kuijpers MJ, Pozgajova M, Cosemans JM, et al. Role of murine integrin alpha2beta1 in thrombus stabilization and embolization: contribution of thromboxane A2. Thromb Haemost 2007; 98(5): 1072-80.

32. Faivre S, Demetri G, Sargent W, et al. Molecular basis for sunitinib efficacy and future clinical development. Nat Rev Drug Discov 2007; 6(9): 734-45. 


\section{Chapter 4}

33. Karaman MW, Herrgard S, Treiber DK, et al. A quantitative analysis of kinase inhibitor selectivity. Nat Biotechnol 2008; 26(1): 127-32.

34. Jooss NJ, De Simone I, Provenzale I, et al. Role of Platelet Glycoprotein VI and Tyrosine Kinase Syk in Thrombus Formation on Collagen-Like Surfaces. Int J Mol Sci 2019; 20(11).

35. Auger JM, Kuijpers MJ, Senis YA, et al. Adhesion of human and mouse platelets to collagen under shear: a unifying model. FASEB J 2005; 19(7): 825-7.

36. Rainer PP, Doleschal B, Kirk JA, et al. Sunitinib causes dose-dependent negative functional effects on myocardium and cardiomyocytes. BJU Int 2012; 110(10): 1455-62.

37. Diaz-Ricart M, Palomo M, Fuste B, et al. Inhibition of tyrosine kinase activity prevents the adhesive and cohesive properties of platelets and the expression of procoagulant activity in response to collagen. Thromb Res 2008; 121(6): 873-83.

38. van Geffen JP, Kleinegris MC, Verdoold R, et al. Normal platelet activation profile in patients with peripheral arterial disease on aspirin. Thromb Res 2015; 135(3): 513-20.

39. Li R, Diamond SL. Detection of platelet sensitivity to inhibitors of COX-1, P2Y(1), and P2Y(1)(2) using a whole blood microfluidic flow assay. Thromb Res 2014; 133(2): 203-10.

40. Hosokawa K, Ohnishi T, Sameshima H, et al. Analysing responses to aspirin and clopidogrel by measuring platelet thrombus formation under arterial flow conditions. Thromb Haemost 2013; 109(1): 102-11.

41. Bossavy JP, Thalamas C, Sagnard L, et al. A double-blind randomized comparison of combined aspirin and ticlopidine therapy versus aspirin or ticlopidine alone on experimental arterial thrombogenesis in humans. Blood 1998; 92(5): 1518-25.

42. Cadroy Y, Bossavy JP, Thalamas C, et al. Early potent antithrombotic effect with combined aspirin and a loading dose of clopidogrel on experimental arterial thrombogenesis in humans. Circulation 2000; 101(24): 2823-8.

43. Mendolicchio GL, Zavalloni D, Bacci M, et al. Variable effect of P2Y12 inhibition on platelet thrombus volume in flowing blood. J Thromb Haemost 2011; 9(2): 373-82.

44. Andre P, LaRocca T, Delaney SM, et al. Anticoagulants (thrombin inhibitors) and aspirin synergize with P2Y12 receptor antagonism in thrombosis. Circulation 2003; 108(21): 2697-703.

45. Sakakibara M, Goto S, Eto K, et al. Application of ex vivo flow chamber system for assessment of stent thrombosis. Arterioscler Thromb Vasc Biol 2002; 22(8): 1360-4.

46. Ninomoto J, Mokatrin A, Kinoshita T, et al. Effects of ibrutinib on in vitro platelet aggregation in blood samples from healthy donors and donors with platelet dysfunction. Hematology 2020; 25(1): 112-7.

47. Sheth RA, Niekamp A, Quencer KB, et al. Thrombosis in cancer patients: etiology, incidence, and management. Cardiovasc Diagn Ther 2017; 7(Suppl 3): S178-s85.

48. Hisada Y, Mackman N. Cancer-associated pathways and biomarkers of venous thrombosis. Blood 2017; 130(13): 1499-506.

49. Sabrkhany S, Kuijpers MJE, van Kuijk SMJ, et al. A combination of platelet features allows detection of early-stage cancer. Eur J Cancer 2017; 80: 5-13.

50. Best MG, Sol N, Kooi I, et al. RNA-Seq of tumor-educated platelets enables blood-based pan-cancer, multiclass, and molecular pathway cancer diagnostics. Cancer Cell 2015; 28(5): 666-76. 


\section{Supplemental data}

\section{Supplemental Table}

Supplemental Table 4.1. Characteristics of cancer patients

\begin{tabular}{|c|c|c|c|c|c|c|}
\hline $\begin{array}{l}\text { Patient } \\
\text { no. }\end{array}$ & Gender & $\begin{array}{l}\text { Age } \\
\text { (year) }\end{array}$ & Cancer type & $\begin{array}{c}\text { Chemotherapy } \\
\text { treatment }\end{array}$ & $\begin{array}{l}\text { Other drug } \\
\text { treatment }(\mathrm{mg} / \mathrm{d})\end{array}$ & $\begin{array}{l}\text { Platelet count } \\
\qquad\left(\times 10^{9} / \mathrm{L}\right)\end{array}$ \\
\hline 1 & Female & 71 & Lung & NA & NA & 164 \\
\hline 2 & Male & 72 & Lung & Pembrolizumab & Aspirin (100) & 105 \\
\hline 3 & NA & NA & Lung & NA & NA & 203 \\
\hline 4 & Male & 76 & Neuroglioblastoma & - & - & 118 \\
\hline 5 & Female & 45 & Neuroglioblastoma & - & - & 93 \\
\hline 6 & Male & 81 & Neuroglioblastoma & - & Fondaparinux (5) & 62 \\
\hline 7 & Male & 76 & Pancreatic & - & - & 248 \\
\hline 8 & Male & 82 & Pancreatic & - & Dabigatran & 168 \\
\hline 9 & Male & 67 & Pancreatic & $\begin{array}{c}\text { Gemcitabine } \\
\text { Abraxane }\end{array}$ & 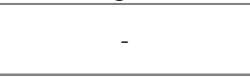 & 198 \\
\hline 10 & Female & 61 & Ovarian & $\begin{array}{c}\text { Oxaliplatin } \\
\text { CPL }\end{array}$ & - & 301 \\
\hline 11 & Female & 30 & $\begin{array}{l}\text { Paraganglioma } \\
\text { (Neuroendocrine } \\
\text { Neoplasm) }\end{array}$ & - & - & 294 \\
\hline
\end{tabular}

NA: Not available; CPL: Caelyx PEGylated liposomal; PEG: Polyethylene glycol

\section{Supplemental Figures}

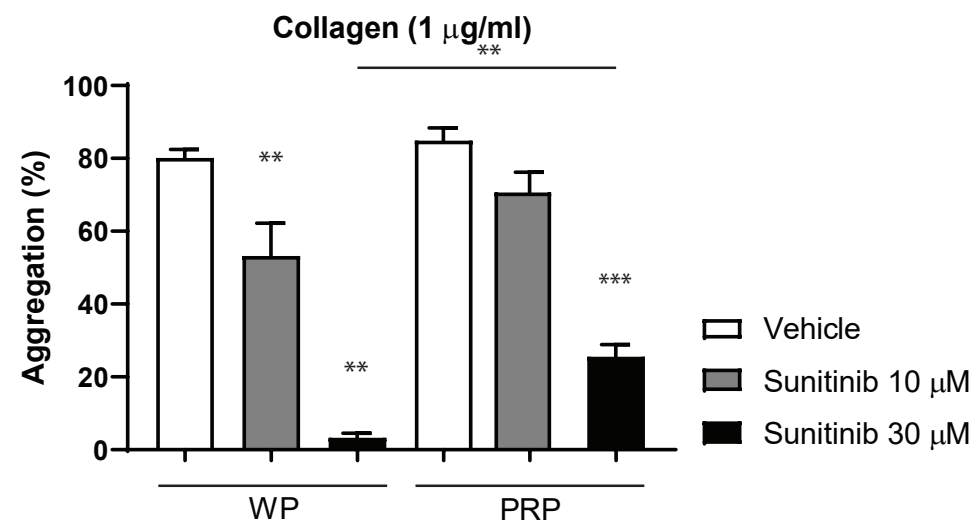

Supplemental Figure 4.1. Inhibition of aggregation with sunitinib is stronger in washed platelets compared to PRP. Washed platelets or PRP $\left(250 \times 10^{\%} / \mathrm{L}\right)$ from healthy volunteers was incubated with vehicle $(<0.1 \%$ DMSO) or sunitinib (10 or $30 \mu \mathrm{M}$ ) for 10 minutes at $37^{\circ} \mathrm{C}$. Shown are the percentages of aggregation of washed platelets (left) or PRP (right) in absence (white bar) or presence of sunitinib (grey / black bars) in response to collagen $(1 \mathrm{\mu g} / \mathrm{mL})$. Data are represented as means + SEM $(\mathrm{n}=4-10),{ }^{*} \mathrm{p}<0.01, * * * \mathrm{p}<0.001$. 

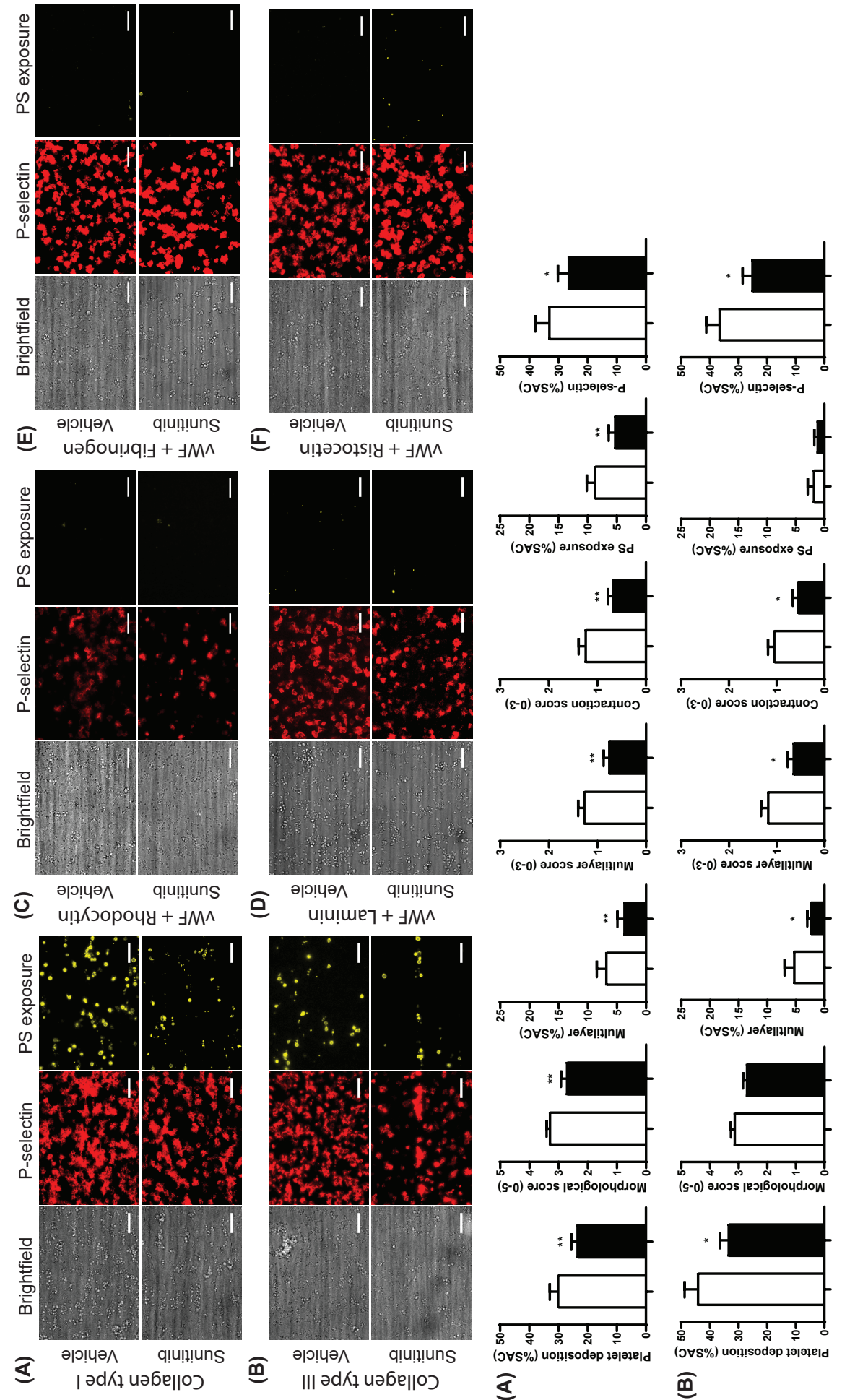

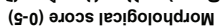
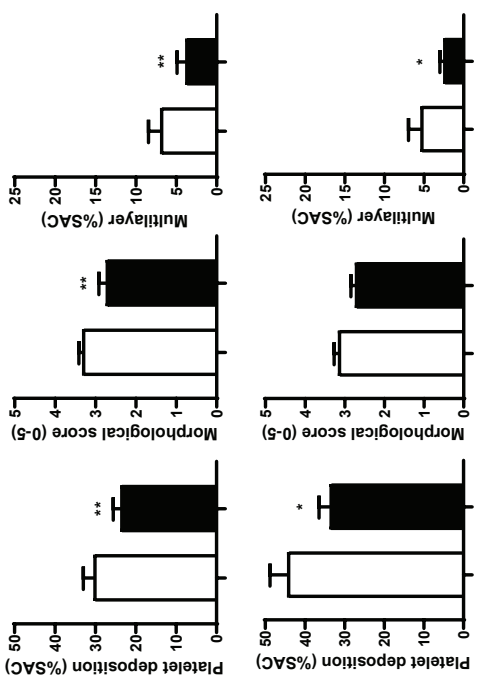

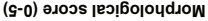

$\varangle$

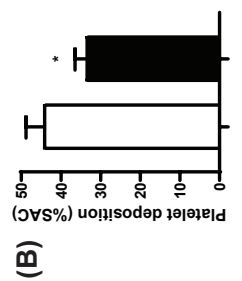




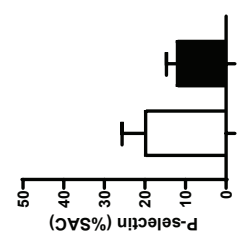

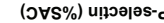

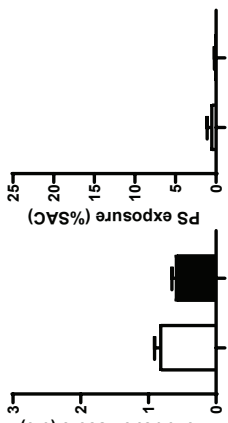

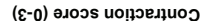

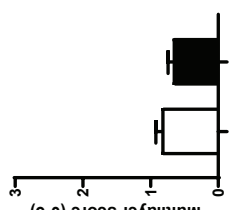

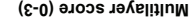

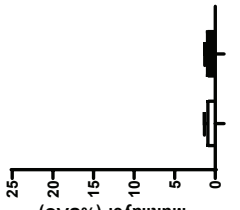

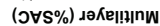

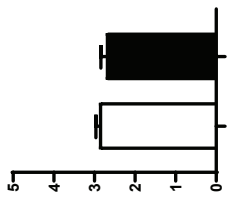

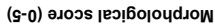

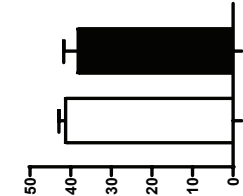

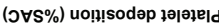
Q

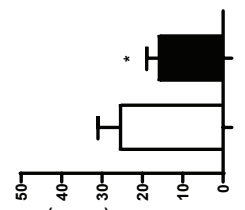

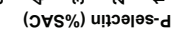
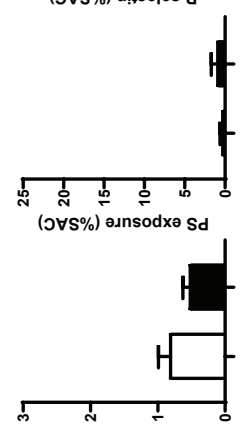

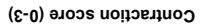
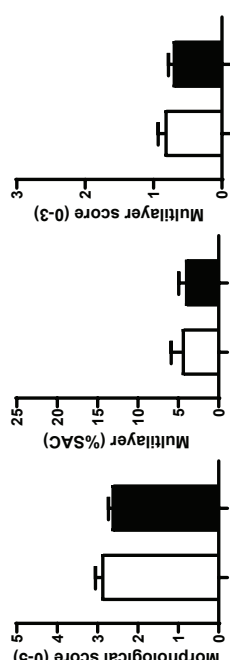

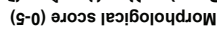

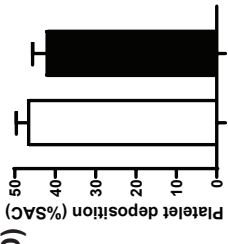

Q

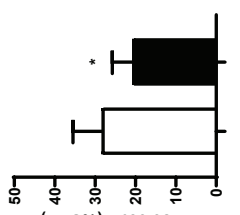

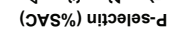

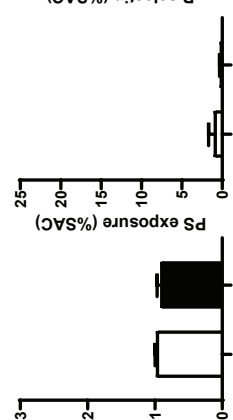

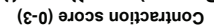

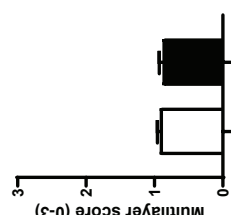

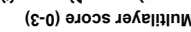

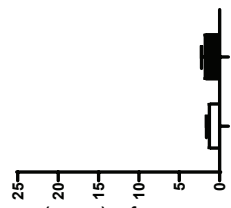

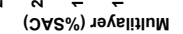

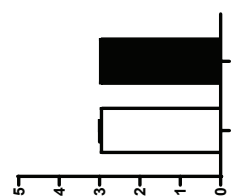

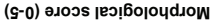

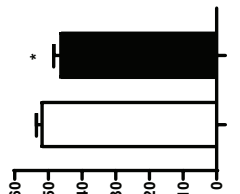

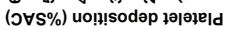
巴ᄄ

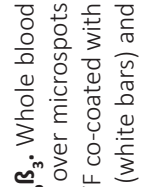

$\sigma^{\underline{\underline{\rho}}}$ is

응 퐁

ली-

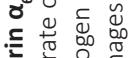

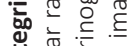

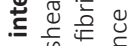

高贾产

(1)

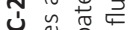

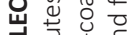

U.

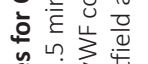

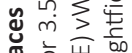

要 就紫

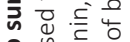

\&

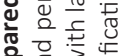

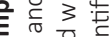

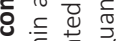

ป

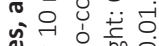

过 훈

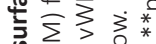

t艹 응

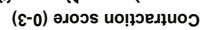

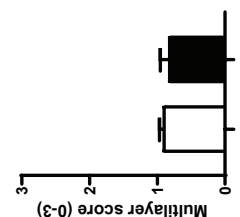

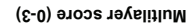

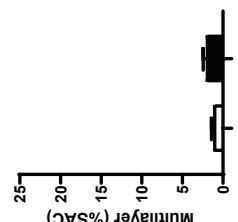

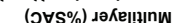

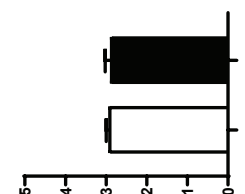

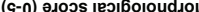

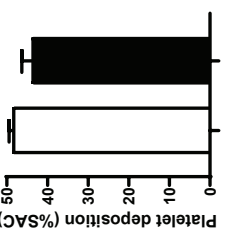

뜨 
(A)

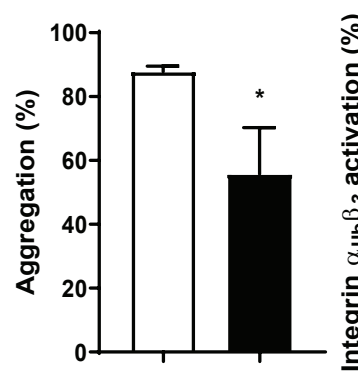

(B)

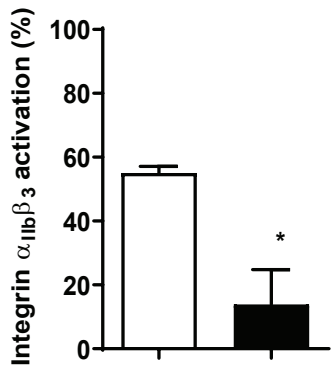

(C)

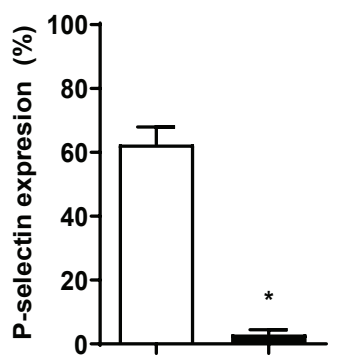

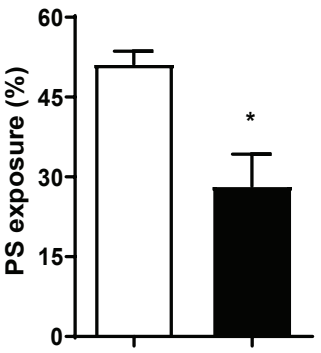

Supplemental Figure 4.3. Sunitinib inhibits CLEC-2 induced aggregation and activation in washed platelets. (A) Washed platelets $(250 \times 10 \%$ L) from healthy volunteers were incubated with vehicle $(<0.1 \% \mathrm{DMSO})$ or sunitinib $(10 \mathrm{or} 30 \mu \mathrm{M})$ for 10 minutes at $37^{\circ} \mathrm{C}$. Shown are the percentages of aggregation in the absence (white bar) or presence of sunitinib (black bars) in response to rhodocytin $(1 \mathrm{\mu g} / \mathrm{mL})$. (B) Washed platelets $\left(100 \times 10^{\%} / \mathrm{L}\right)$ were pre-incubated with vehicle $(<0.1 \%$ DMSO) or sunitinib $(10 \mu \mathrm{M})$ and stimulated rhodocytin $(1 \mu \mathrm{g} / \mathrm{mL})$ for 15 minutes and simultaneously stained for activated integrin $\alpha_{11 b} \beta_{3}$ and $\alpha$-granule secretion (P-selectin). Histograms show the percentages of platelets binding FITC-labelled PAC-1 monoclonal antibody or AF647-labelled anti-human CD62 mAb. (C) Isolated platelets from healthy volunteers were incubated with vehicle $(<0.1 \% \mathrm{DMSO})$ or sunitinib $(10 \mu \mathrm{M})$ for 10 minutes at $37^{\circ} \mathrm{C}$. Platelets $\left(100 \times 10^{9} / \mathrm{L}\right)$ were activated in the presence (black bars) or absence of sunitinib (white bars) by rhodocytin $(5 \mu \mathrm{g} / \mathrm{mL})$ in combination with thrombin $(4$ $\mathrm{nM}$ ) and incubated for 60 minutes at $37^{\circ} \mathrm{C}$. PS exposure was detected by FITC-conjugated annexin A5 and measured by flow cytometry. Data are represented as means + SEM $(n=4-6),{ }^{*} p<0.05$. 
(A)
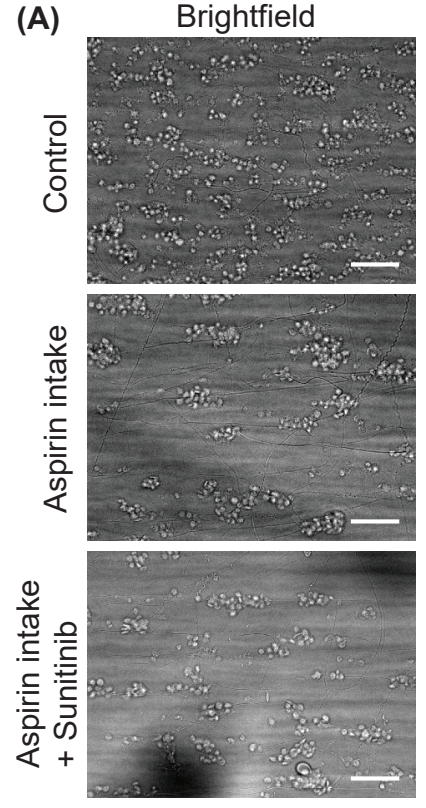

(B) $\mathrm{i}$
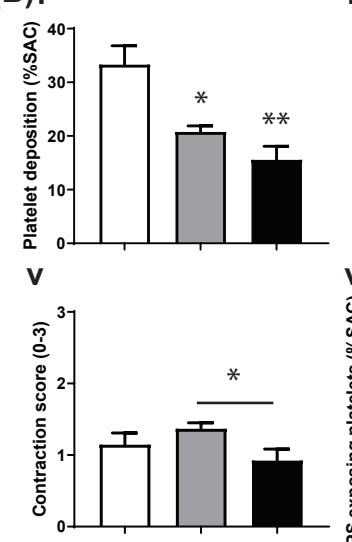

ii
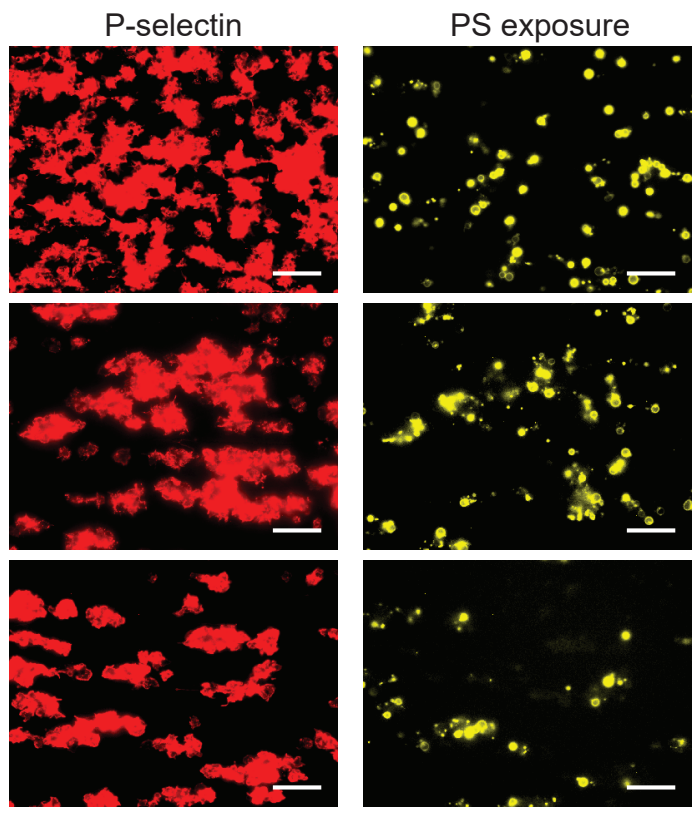

iii
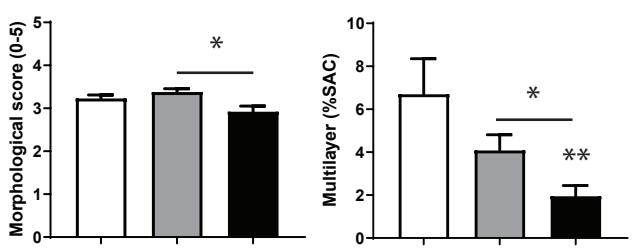

iv

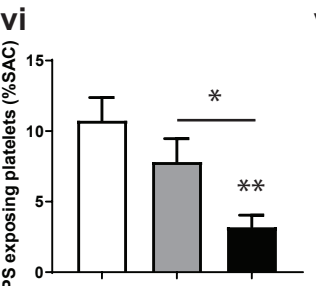

vii
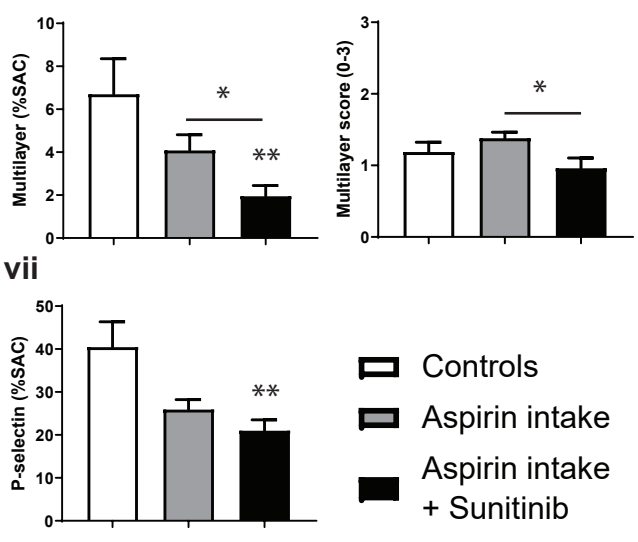

Supplemental Figure 4.4. Aspirin intake enhances the effect of sunitinib in ex vivo thrombus formation on collagen in whole blood. Recalcified whole blood of healthy volunteers or healthy volunteers with single dose of aspirin was treated in vitro with vehicle or sunitinib $(30 \mu \mathrm{M})$ for $10 \mathrm{~min}$. Subsequently, blood was perfused over a collagen surface at $1000 \mathrm{~s}^{-1}$ for 3.5 minutes, followed by perfusion with buffer supplemented with AF568-annexin A5 and AF647- $\alpha$-CD62P to visualize PS exposure and P-selectin expression, respectively. (A) Representative brightfield and fluorescent images. (B) Quantification of brightfield images of (i) platelet deposition, (ii) morphological score, (iii) multilayer (\%SAC), (iv) multilayer score, (v) contraction score, and fluorescence images of (vi) PS exposure and (vii) P-selectin expression of vehicle (white bar), aspirin intake (dark grey bars), or aspirin intake with in vitro sunitinib (black bar). Data are represented as means + $\operatorname{SEM}(n=3-6), * p<0.05, * * p<0.01$. 


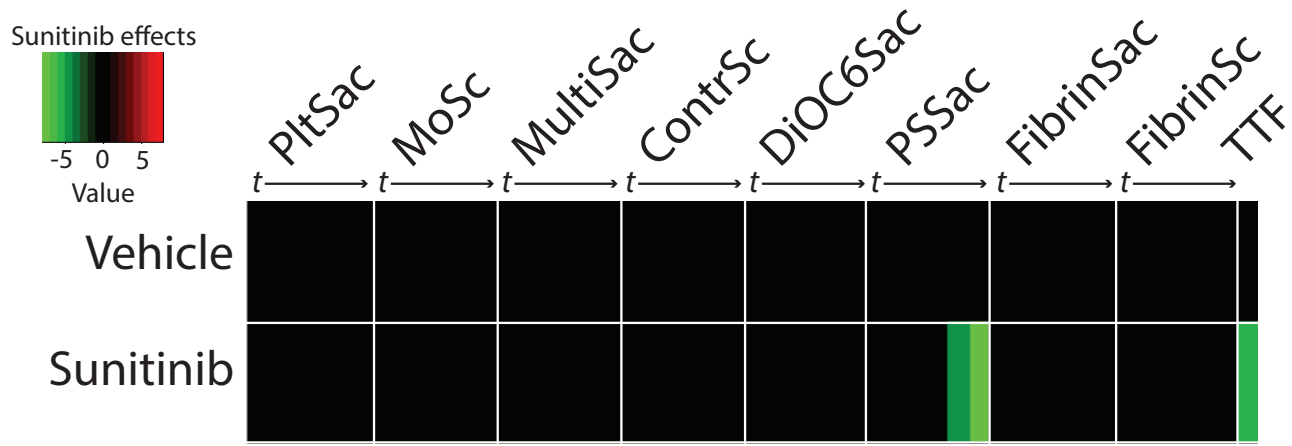

Supplemental Figure 4.5. The effects of sunitinib on platelet-dependent fibrin formation under flow. Whole blood from healthy volunteers was preincubated with vehicle $(<0.1 \%$ DMSO) or sunitinib $(30 \mu M)$ for 10 min. Subsequently, the blood was supplemented with DiOC6, AF568-annexin A5 and AF647- $\alpha$-fibrinogen to visualize platelet deposition, PS-exposure and fibrin formation, respectively. Blood samples were co-perfused with $\mathrm{CaCl}_{2} / \mathrm{MgCl}_{2}$ over collagen type I co-coated with TF microspot at a wall shear rate of $1000 \mathrm{~s}^{-1}$. Subtraction heatmaps representing the significant effects of normalized values of the parameters. Controls with addition of vehicle $(<0.1 \% \mathrm{DMSO})$ were set at 0 for reference. Effects were filtered for significant alterations by addition of sunitinib $(p \leq 0.05)$. 
(A)

Brightfield
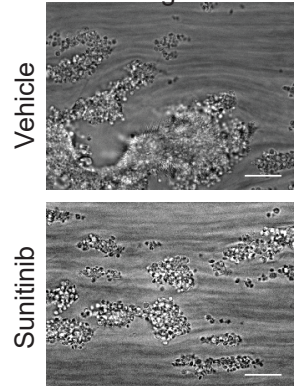

(B) $\mathrm{i}$
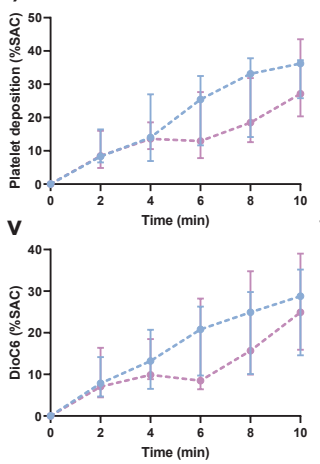

Sunitinib effects

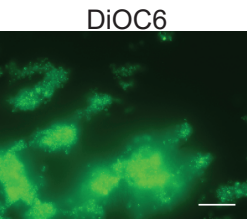

PS exposure
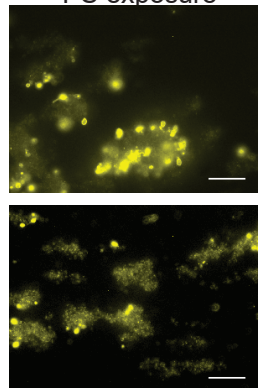

iii
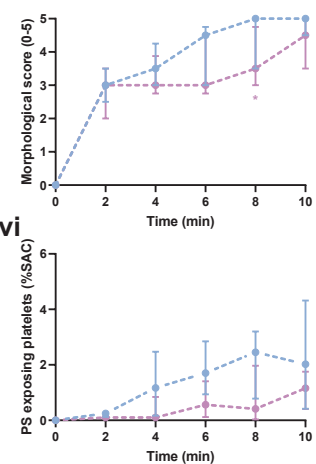
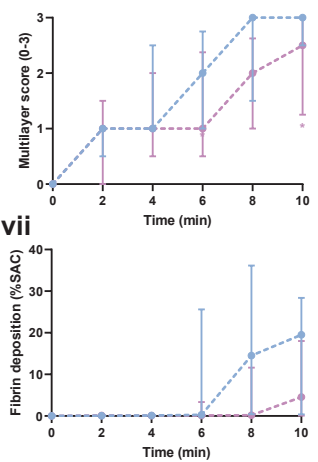

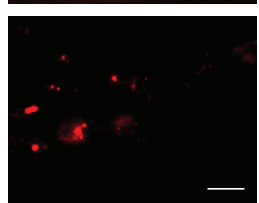

iv
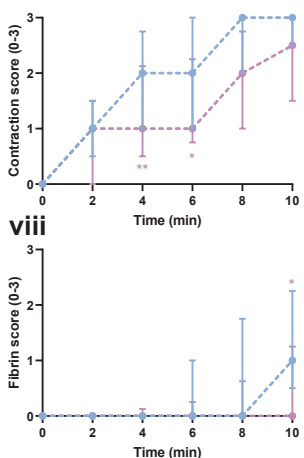

(C)

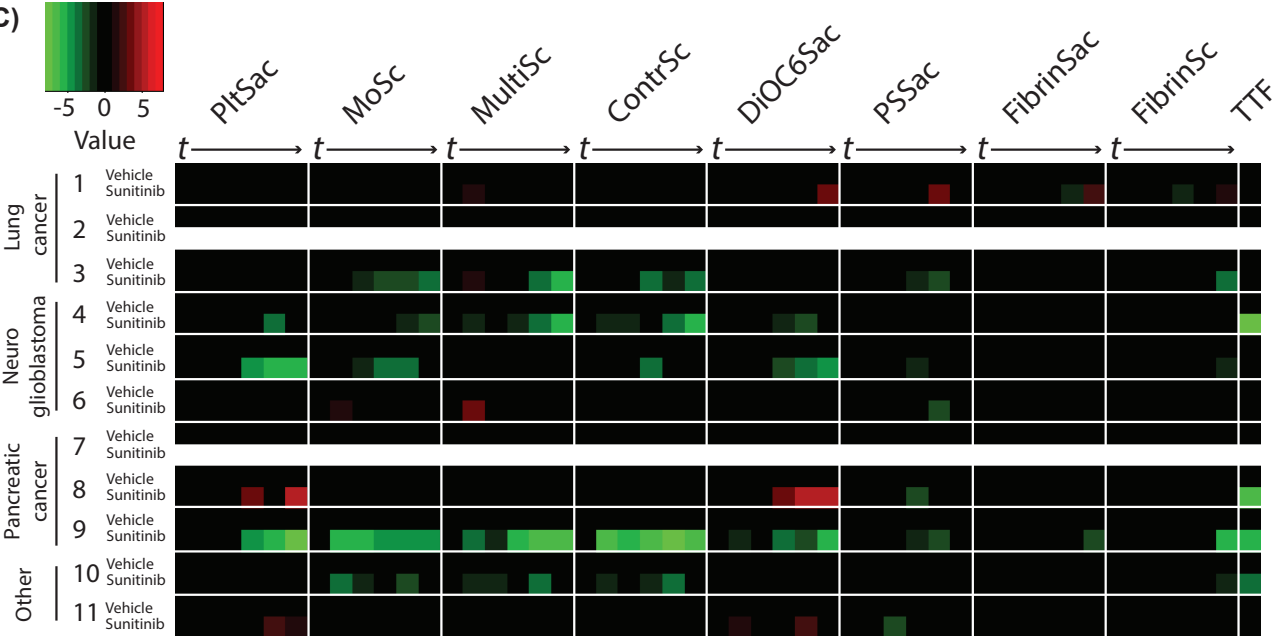

Supplemental Figure 4.6. Sunitinib reduces platelet-dependent thrombus-fibrin formation under flow in whole blood from cancer patients. Citrated whole blood from cancer patients (suppl. Table I) was treated in vitro with vehicle $(<0.01 \%$ DMSO) or sunitinib $(30 \mu \mathrm{M})$ for $10 \mathrm{~min}$, followed by addition of DiOC6 (platelet deposition), AF568-annexin A5 (PSexposure) and AF647- $\alpha$-fibrinogen (fibrin). Blood was co-perfused with $\mathrm{CaCl}_{2} / \mathrm{MgCl}_{2}$ over collagen type I co-coated with TF at a wall shear rate of $1000 \mathrm{~s}^{-1}$. (A) Representative brightfield and fluorescent images taken after $10 \mathrm{~min}$ of flow. (B) Kinetic analysis of brightfield images of (i) platelet deposition (PItSac), (ii) morphological score (MoSc), (iii) multilayer score (MultiSc), and (iv) contraction score (ContrSc) and of fluorescence images of (v) DiOC6 platelet deposition (DiOC6Sac), (vi) PS exposure (PSSac), (vii) fibrin deposition (FibrinSac), and (viii) fibrin score (FibrinSc). Data are represented as median \pm interquartile range $(n=9-11),{ }^{*} p<0.05,{ }^{* *} p<0.01$. (C) Subtraction heatmap representing the effect of sunitinib in individual cancer patients. Vehicle treated blood of cancer patients was set at 0 for reference to in vitro addition of sunitinib. Effects were filtered for changes greater than the $1 \times$ SD range of the different platelet parameters. 

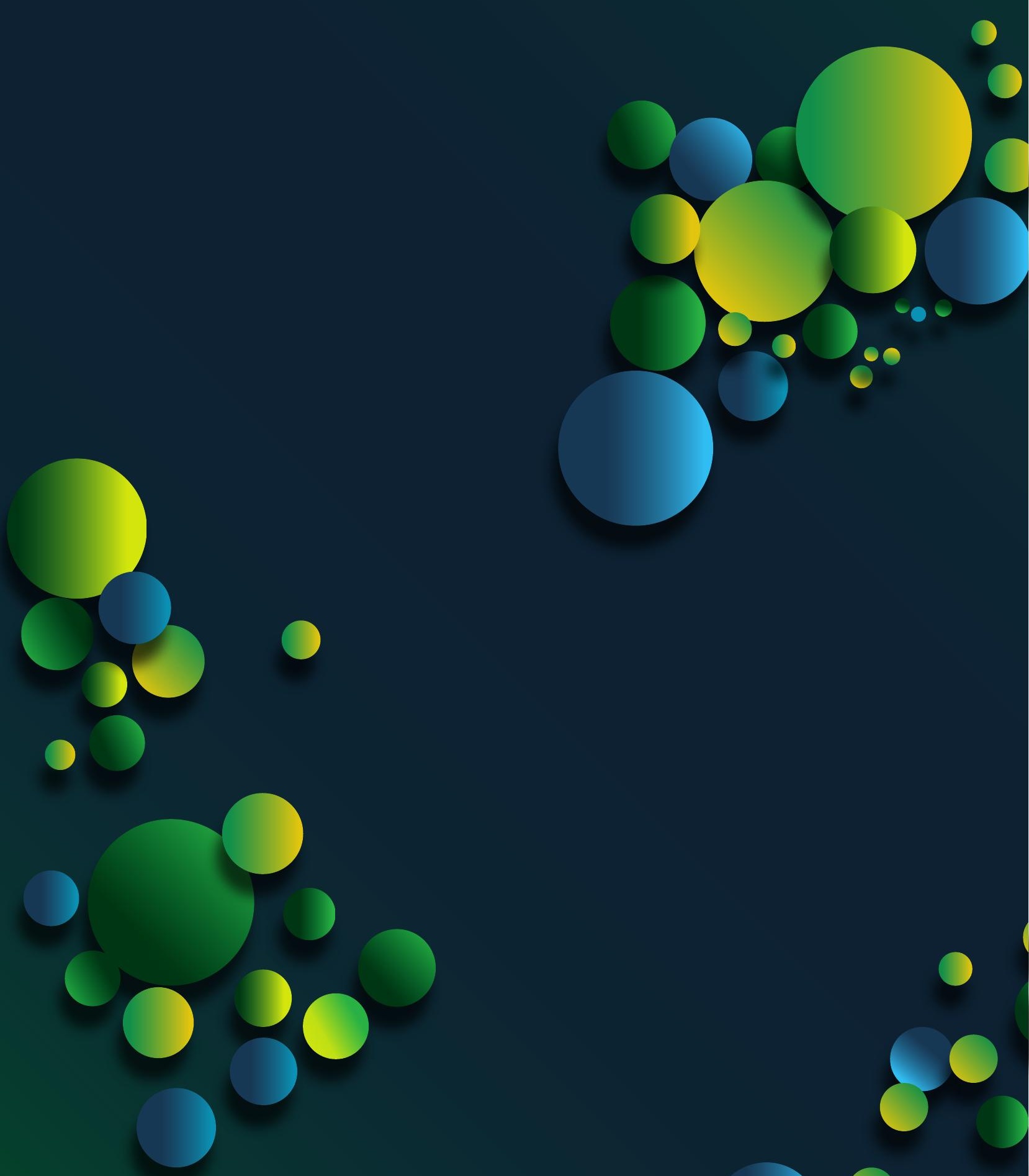


\section{Chapter 5}

Quantitative and qualitative changes in platelet traitsofsunitinib-treated patientswith renal cell carcinoma in relation to circulating sunitinib levels: a proof-of-concept study

Tullemans BME, Brouns SLN, Swieringa F, Sabrkhany S, van den Berkmortel FWPJ, Peters NJB, de Bruijn P, Koolen SLW, Heemskerk JWM, Aarts MJB and Kuijpers MJE 


\section{Chapter 5}

\section{Abstract}

Background: Tyrosine kinase inhibitors (TKIs), such as sunitinib, are used for cancer treatment, but may also affect platelet count and function with possible hemostatic consequences. Here, we investigated how patient treatment with the TKI sunitinib affected quantitative and qualitative platelet traits as a function of the sunitinib level and the occurrence of bleeding.

Methods: Blood was collected from 20 metastatic renal cell carcinoma (mRCC) patients before treatment, and at 2 weeks, 4 weeks and 3 months after sunitinib administration. We measured blood cell counts, platelet aggregation, and the concentrations of sunitinib as well as its N-desethyl metabolite in plasma, serum and isolated platelets. Progression of disease (PD) and bleeding were monitored after 3 months.

Results: In sunitinib-treated mRCC patients, concentrations of ( $\mathrm{N}$-desethyl)sunitinib in plasma and serum were highly correlated. In the patients' platelets the active metabolite levels were relatively increased as compared to sunitinib. On average, a sustained reduction in platelet count was observed on-treatment, which was significantly related to the inhibitor levels in plasma/serum. Principal component and correlational analysis showed that the (N-desethyl-) sunitinib levels in plasma/serum were linked to a reduction in both platelet count and collageninduced platelet aggregation. The reduced aggregation associated in part with reported bleeding, but did not correlate to PD.

Conclusions: The sunitinib-induced reduction in quantitative and qualitative platelet traits may reflect the effective sunitinib levels in the patient. These results may serve as a proof-ofprinciple for other TKI-related drugs, where both platelet count and functions are affected, which could be used therapeutic drug monitoring. 


\section{Introduction}

In the past two decades, over 40 tyrosine kinase inhibitors (TKIs) have been developed and approved for the treatment of many cancer types ${ }^{1}$. The majority of these drugs target the receptor-linked tyrosine kinases for growth factors, e.g., receptors for vascular endothelial growth factor, and/or differentiation/proliferation factors (e.g., Flt, Fms, Kit, and Ret). Several other TKIs target intracellular tyrosine kinases (Abl, B-Raf, Btk, Itk, Src-family kinases and Syk). However, in spite of the intended specific targets, most TKIs used for treatment also have broad off-target effects, invariably affecting a range of protein tyrosine kinases. It is debated whether these offtarget effects help to improve progression-free survival (PFS) and overall survival (OS) of the treated patients ${ }^{2}$.

Until shortly, the survival of metastatic clear cell renal cell carcinoma (ccRCC) patients was poor with a 5 -year survival rate of $12 \%^{3}$. However, in recent years treatment options have increased by the availability of immune- and targeted therapies, using anti-PD1 and anti-CTLA4 antibodies, as well as new TKIs. Randomized controlled trials testing the targeted therapies showed an overall improvement in response rates, PFS and OS, especially for the intermediate and poor risk groups ${ }^{4-7}$. For the favorable risk patients, it appeared that sunitinib, a broad-spectrum TKI, was superior to the immuno-agents nivolumab/ipilimumab, and was equivalent to pembrolizumab plus axitinib $b^{4,5}$. Therefore, sunitinib has become a mainstay not only for first-line treatment in this particular risk group, but also in second line treatment after nivolumab/ipilimumab.

Upon activation of platelets via non-G-protein coupled receptors, tyrosine phosphorylation, via Src-family kinases and Syk, is one of the key signal transduction mechanisms ${ }^{8}$. Especially, platelet activation via the collagen receptor glycoprotein (GP) VI fully relies on this tyrosine kinase pathway. Clinical studies have shown that several TKIs, next to affecting platelet function, also affect platelet count. Both effects on platelets can contribute to the increased bleeding risk, regularly observed in patients treated with $\mathrm{TKI}^{1,9}$. Also for sunitinib treatment, mild bleeding diathesis has been described as a side effect. This was observed as epistaxis, or mucocutaneous and gastrointestinal bleeding, which occurred in approximately $19 \%$ of the sunitinib-treated patients ${ }^{10}$.

Recently, we have demonstrated a rapid uptake of sunitinib by platelets, which was accompanied by a reduced collagen receptor-induced aggregation, secretion of $\alpha$-granules and thrombus formation under flow conditions, both in vitro and ex vivo in RCC patients on treatment ${ }^{11}$. Furthermore, we and others have shown that the platelet concentration decreases upon sunitinib treatment ${ }^{11-13}$, an effect that has been postulated as a prognostic factor for the sunitinib treatment response in $\mathrm{RCC}^{12}$. However, to which extent these sunitinib effects are related to each other and to the circulating sunitinib concentration and/or bleeding is unclear.

In the present study with 20 patients, we investigated as a proof-of-concept with regards to other TKIs, whether the effects of sunitinib on quantitative (count) and qualitative (aggregation) 
platelet traits is associated with the circulating inhibitor level. Furthermore, we set out to deduce how this relates to reported bleeding symptoms.

\section{Materials and Methods}

\section{Materials}

Sunitinib malate (Sutent) was provided by Pfizer (New York NY, USA). Bovine serum albumin (BSA), D(+)-glucose, and apyrase were purchased from Sigma-Aldrich (Saint Louis MO, USA). Horm collagen type I was from Takeda (Hoofddorp, the Netherlands). Ilomedin (iloprost) injection fluid was obtained from Bayer (Mijdrecht, the Netherlands).

\section{Study population and blood collection}

The study was performed in accordance with the declaration of Helsinki and approved by the local medical ethical committee of Maastricht University Medical Center+ (MUMC+). Full informed consent was obtained from all participants. Blood samples were collected from 20 patients diagnosed with metastatic renal cell carcinoma (mRCC) at the Department of Medical Oncology of MUMC+ (Maastricht, the Netherlands), Zuyderland Medical Centre (Sittard-Geleen, the Netherlands) and SJG Hospital (Weert, the Netherlands). Blood was also collected from 10 healthy donors of similar age and gender. Patients were included, when eligible for treatment with sunitinib as a single agent ( $50 \mathrm{mg} /$ day) with a treatment schedule of 4 weeks on and 2 weeks off medication. Patient blood samples were collected at four different timepoints: (i) before start with sunitinib, and after (ii) 2 weeks, (iii) 4 weeks and (iv) 3 months of sunitinib administration.

Blood samples were obtained from the antecubital vein. At each timepoint; $10 \mathrm{ml}$ was collected using a vacuum container containing 3.2\% trisodium citrate, and a second blood sample of $10 \mathrm{ml}$ was collected in a Clot Activator Tube (CAT) Serum Separator (Greiner Bio-One, Alphen a/d Rijn, the Netherlands). Blood cell counts and haematological parameters were assessed in patient and healthy control samples, using a Sysmex XP300 (Kobe, Japan).

\section{Reporting of bleeding}

Patients were asked to fill out a self-assessment bleeding form after 2 weeks, 4 weeks and 3 months of sunitinib administration, as was used earlier ${ }^{14}$. The physicians provided additional information with regards to bleeding. Any bleeding was scored as 1 , no bleeding was scored as 0 .

\section{Response evaluation}

A response to sunitinib treatment was defined as partial response (PR), stable disease (SD) or progressive disease (PD) according to Response Evaluation Criteria in Solid Tumors (RECIST) 1.1 guidelines, based on a CT scan performed 3 months after initiating sunitinib, as compared to the CT scan prior to treatment. For some of the 20 patients, values were only collected at 
timepoints 0 and 2 weeks. Missing values at 4 weeks and/or 3 months were due to dose reduction (in 3 patients, after severe side effects of medication), hospital admission as a result of thrombocytopenia requiring blood transfusion (2 patients) or death (4 patients).

\section{Platelet isolation}

Platelets were isolated from whole blood, as described previously ${ }^{15}$. Platelet-rich plasma (PRP) was isolated from citrate-anticoagulated blood by centrifugation at $240 \mathrm{~g}$ for 15 minutes. The PRP was supplemented with 1:10 acidic citrate dextrose (ACD; 80 mM trisodium citrate, 52 $\mathrm{mM}$ citric acid and $180 \mathrm{mM}$ glucose) and centrifuged for 2 minutes at 2,230 g. The supernatant plasma was retained in case of patient samples, and was further processed as described below. The platelet pellet was resuspended into Hepes buffer pH $6.6(10 \mathrm{mM}$ Hepes, $136 \mathrm{mM} \mathrm{NaCl}, 2.7$ $\mathrm{mM} \mathrm{KCl}$ and $2 \mathrm{mM} \mathrm{MgCl}_{2}$ ) supplemented with $5 \mathrm{mM}$ glucose and $0.1 \%$ bovine serum albumin (BSA). After addition of 1:15 ACD and $1 \mathrm{U} / \mathrm{mL}$ apyrase, the platelets were centrifuged for 2 minutes at 2,230 $\mathrm{g}$, followed by resuspension into Hepes buffer $\mathrm{pH} 7.45$ with $5 \mathrm{mM}$ glucose and $0.1 \%$ BSA. Platelet count was determined using a Sysmex XP300 (Kobe, Japan) and adjusted as stated per assay.

\section{Patient blood sample preparations}

Serum was isolated from CAT tubes by centrifugation at 2,200 $\mathrm{g}$ for 10 minutes at room temperature. Both serum and plasma (obtained from PRP) were centrifuged a second time at $22,500 \mathrm{~g}$ for 5 minutes to remove possible debris. The collected plasma and serum samples were frozen, and stored at $-80^{\circ} \mathrm{C}$ until further use.

Isolated platelets $\left(250 \times 10^{9}\right.$ platelets/L) were incubated for 5 minutes at room temperature with $50 \mathrm{nM}$ iloprost, and centrifuged for 2 minutes at 2,230 g. Supernatant was discarded, and the pellet was frozen and stored at $-80^{\circ} \mathrm{C}$ until further use.

\section{Light transmission aggregometry}

Washed platelets $\left(250 \times 10^{9}\right.$ platelets/L) were incubated for 5 minutes at $37^{\circ} \mathrm{C}$ before stimulation. Aggregation response was induced by addition of $1 \mu \mathrm{g} / \mathrm{mL}$ collagen type I, except for patients with anti-platelet drugs where aggregation was induced by $5 \mu \mathrm{g} / \mathrm{mL}$ collagen. The collagen concentration was kept the same at all timepoints per patient. Platelet aggregation was recorded using a Chronolog optical aggregometer (Havertown PA, USA), and maximum aggregation amplitude was quantified at 8 minutes after collagen addition.

\section{Pharmacokinetic analysis of sunitinib in plasma and serum samples}

Sunitinib and the N-desethyl metabolite (SU12662) were quantified, as described previously ${ }^{16}$ in plasma, serum and platelet pellets obtained from patients after 2 and 4 weeks on 
sunitinib treatment. The concentration of either compound in isolated platelets was corrected for the number of platelets in the pellet, and normalized to ng per $2.5 \times 10^{8}$ platelets.

\section{Statistical analysis}

Data are presented as median \pm interquartile ranges. Datasets within patients were compared using the Wilcoxon matched pairs test, whereas comparisons to controls were determined using the Kruskal-Wallis test. Correlation analysis was performed using a nonparametric Spearman correlation (2-tailed) using GraphPad Prism 8 software. To identify associations within the dataset, variables were quantile normalized and a rotated principal component analysis, based on an eigenvalue over 1, was performed using the statistical package for social sciences (SPSS version 24). A P-value less than 0.05 was considered to be statistically significant.

\section{Results}

\section{Patient demographics and clinical characteristics}

In the present study, 20 patients were included with $\mathrm{mRCC}$, who were eligible for treatment with $50 \mathrm{mg}$ sunitinib per day with a treatment schedule of 4 weeks on and 2 weeks off medication (Table 5.1). Median age of the mRCC patients was 65 years (range: 52-83). Of the patients, 6 were females (median age 65; range: 59-83) and 14 were male (median age 65.5; range: 52-80). All patients presented with ccRCC, except for two patients who were diagnosed with papillary RCC. Three patients required dose reduction of sunitinib during the study follow-up, due to side effects (Table 5.1). Four of the patients additionally received daily aspirin as an anti-platelet drug, one of whom received heparin during the 3-month follow-up. Two patients received the cholesterollowering drug atorvastatin, which can also affect platelet function ${ }^{17,18}$. Furthermore, 10 healthy individuals were included with similar median age of 63.5 (range: 53-85), of whom 3 were female.

\section{Plasma and serum levels of sunitinib and N-desethyl-sunitinib are highly correlated, while platelets take up more $\mathrm{N}$-desethyl-sunitinib as compared to sunitinib}

Sunitinib and its active metabolite $\mathrm{N}$-desethyl-sunitinib were determined in both plasma and serum from mRCC patients after 2 and 4 weeks of treatment. The concentration of $\mathrm{N}$-desethyl-sunitinib appeared to be significantly lower in serum and plasma than that of sunitinib (Figure 5.1A vs 5.1B, plasma $\mathrm{P}<0.0001$ and serum $\mathrm{P}<0.0006$ ). Comparison of plasma and serum levels of sunitinib or $\mathrm{N}$-desethyl-sunitinib did not show marked differences at 2 or 4 weeks (Figure 5.1A, B). On the other hand, the concentration of sunitinib, but not of its metabolite, was slightly, but significantly, decreased after 4 weeks in both plasma and serum, as compared to 2 weeks on treatment (Figure 5.1A). This is compatible with a 14-days peak level upon sunitinib administration, after which this reduced to a steady-state level.

Because platelets are known to sequester sunitinib, we also measured the concentrations 


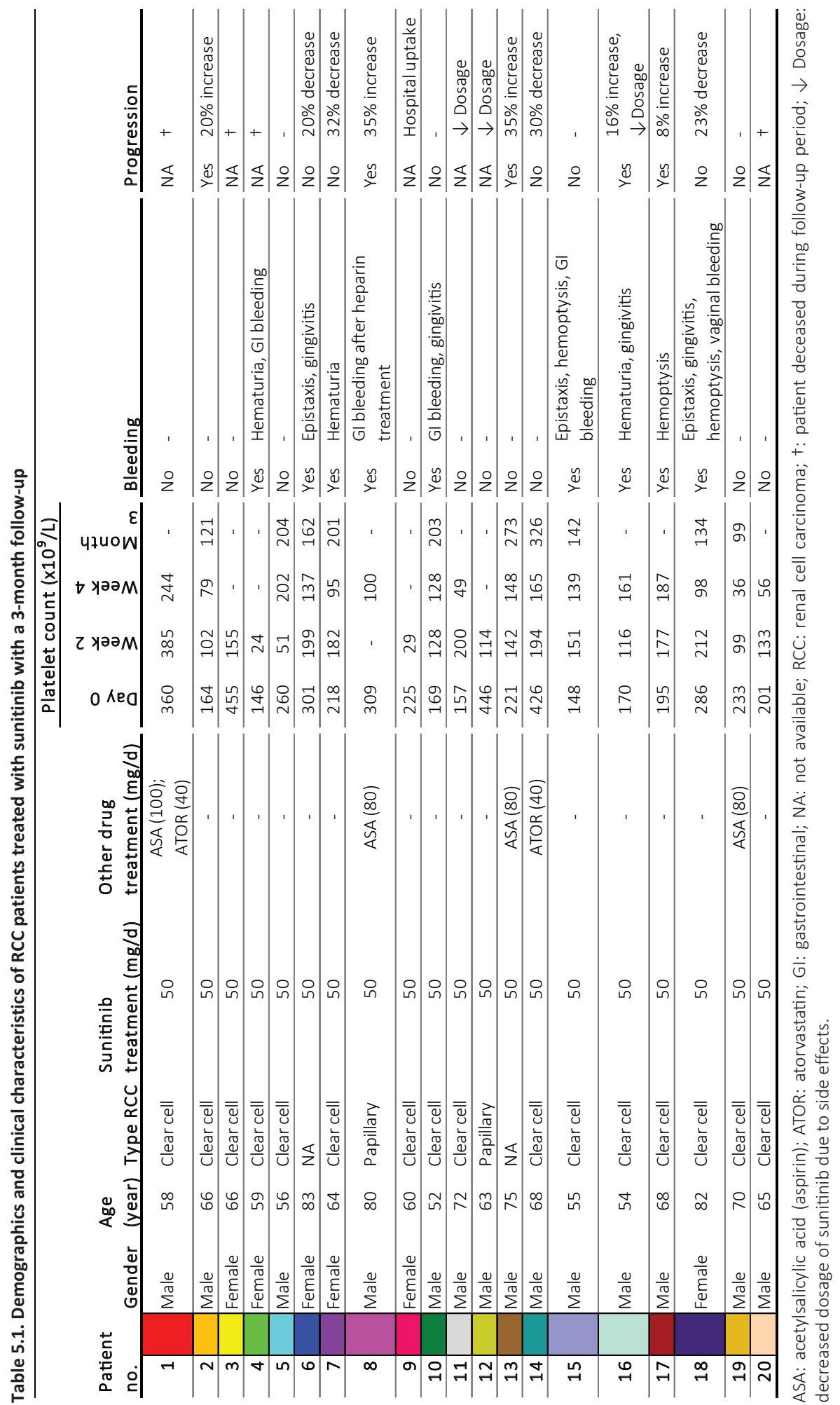


of sunitinib and the active metabolite in isolated platelets from mRCC patients. Markedly, the concentration of the metabolite was significantly higher in platelets after 2 and 4 weeks on treatment, as compared to that of sunitinib (Figure 5.1C). This contrasted to the lower concentration of the metabolite in the plasma and serum samples, suggesting a preferential uptake of the metabolite by platelets. Furthermore, the concentrations of sunitinib and metabolite after 2 and 4 weeks of treatment showed strong correlations between the plasma, serum and platelet samples $(r>0.67033, P<0.01)$.

\section{Prolonged changes in platelet count by sunitinib treatment of $m R C C$ patients are related} to inhibitor levels in plasma and serum

Whole blood cell counts, haemoglobin and haematocrit were determined in blood samples from mRCC patients at four time points (Figure 5.2), i.e., the day before start of sunitinib treatment, after 2 and 4 weeks ( 1 treatment cycle) and after 3 months ( 2 complete cycles). Before start of sunitinib treatment, no significant differences were observed in these parameters

(A)

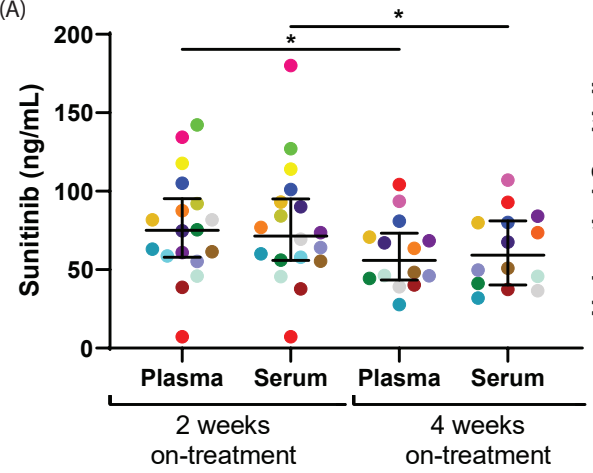

(C)
(B)

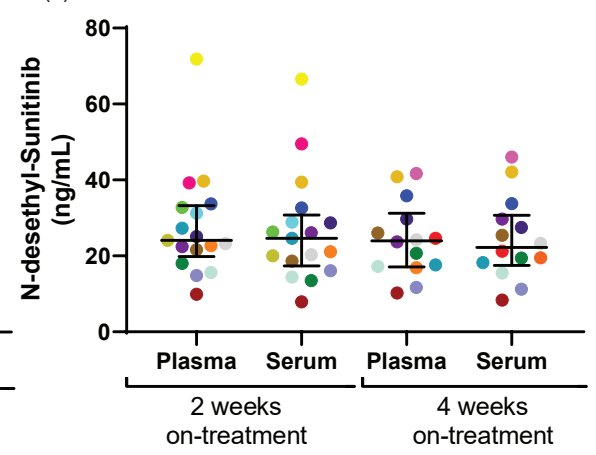

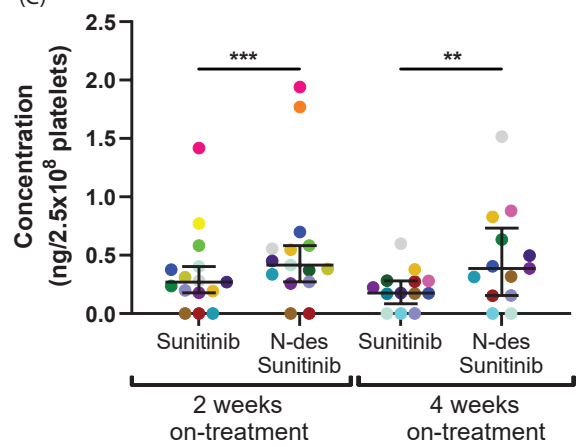

Figure 5.1. Comparison of sunitinib and $\mathrm{N}$-desethyl-sunitinib levels in plasma, serum and isolated platelets from mRCC patients on $\mathbf{2}$ and $\mathbf{4}$ weeks on treatment. The concentration of sunitinib (A) and N-desethyl-sunitinib (B) were measured in plasma and serum samples obtained from mRCC patients at 2 and 4 weeks of sunitinib treatment. (C) The concentrations of both compounds were determined in isolated platelets, normalized to ng per $2.5 \times 10^{8}$ platelets. Individual dots in the scatterplots represent one patient, for color coding see Table 5.1. Lines and error bars represent median \pm interquartile range $(n=14-20) .{ }^{*} P<0.05 .{ }^{* *} P<0.01,{ }^{* * *} P<0.001$ 
between patients and healthy controls (Figure 5.2). However, after 2 weeks of treatment platelet counts were decreased (>10\%) in 15 patients (range: 17-87\% reduction), while 5 patients showed no decrease or even an increase (5-10\%, Figure 5.2A). Seven of the patients who presented a reduced platelet count showed an even further reduction after 4 weeks, which effect for six of them persisted for 3 months. The median platelet concentration of all patients increased after 3 months as compared to 4 weeks, yet was still lower than before the start of treatment (Figure 5.2A). In total, fifteen patients had a platelet count between $24-148 \times 10^{9} / \mathrm{L}$ (Table 5.1), which was below the normal range (150-400×10\%/L, according to the Dutch Society of Hematology, NVH). Of note, 5 patients presented with formal thrombocytopenia $\left(<50 \times 10^{9}\right.$ platelets/L) after 2-4 weeks of treatment. White blood cell (WBC) counts decreased (>10\%) in 13 patients (range: 19-60\% reduction) after 2 weeks of sunitinib treatment (Figure 5.2B), which decrease persisted in most patients for 3 months. According to the Common Terminology Criteria 7 patients presented with grade 2 neutropenia during the study period with no more than 3 patients per time point. No patients had grade 3 or 4 neutropenia. After two weeks of sunitinib administration, overall red
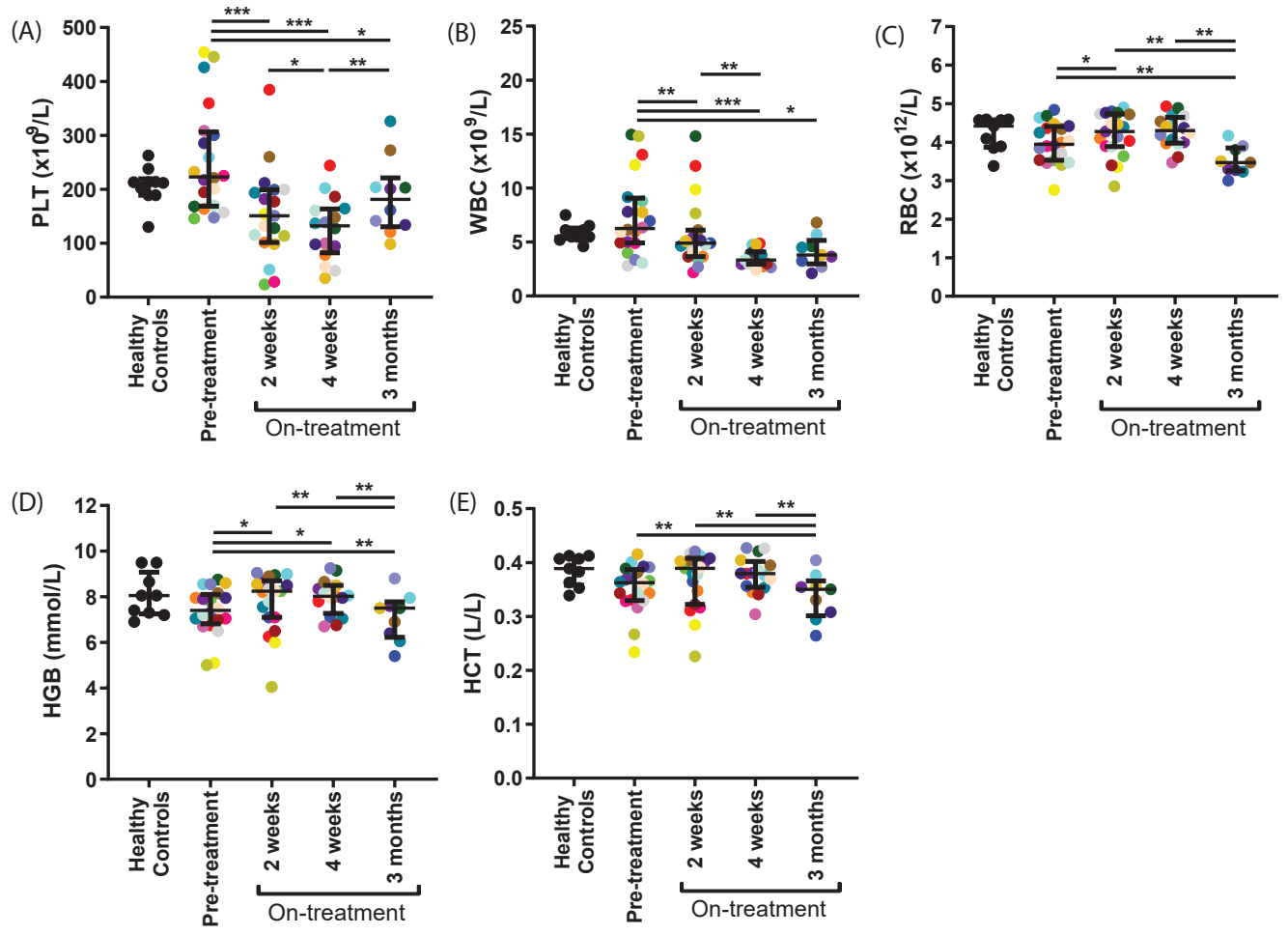

Figure 5.2. Treatment of $\mathrm{mRCC}$ patients with sunitinib decreases platelet count and other hematological parameters over time. Blood was collected from 10 healthy controls and 20 mRCC patients the day before, after 2 weeks, 4 weeks and 3 months on sunitinib treatment. Hematological parameters were measured in whole blood. Scatterplots show (A) platelet count, (B) white blood cell count (WBC), (C) red blood cell count (RBC), (D) hemoglobin concentration (HGB) and (E) hematocrit levels (HCT). Individual dots in the scatterplots represent one patient, for color coding see Table 1. Lines and error bars represent median \pm interquartile range $(n=10-20) .{ }^{*} P<0.05$. ${ }^{*} * P<0.01,{ }^{* * *} P<0.001$. 
blood cell count (RBC) and hemoglobin were slightly, but significantly increased (Figure 5.2C-D). After 4 weeks and 3 months, these values were decreased, as well as the hematocrit (Figure 5.2E).

We examined whether the concentrations of ( $\mathrm{N}$-desethyl-)sunitinib were correlated with the reduction in platelet count. The levels of either compound in plasma and serum appeared to be correlated with the count reductions after 2 weeks treatment (Table 5.2, Figure 5.3A), and for the metabolite this correlation was even stronger after 4 weeks (Table 5.2). For the compound levels in platelets, we noticed a borderline significant negative correlation (Table 5.2, Figure 5.3B). Together, these data suggested that the changes in platelet count are related to the sunitinib levels in plasma and serum, thereby possibly reflecting the effective sunitinib dose in the patient.

Table 5.2. Concentrations of ( $\mathrm{N}$-desethyl-)sunitinib in plasma and serum correlate with changes in platelet count and aggregation in RCC patients at different time points.

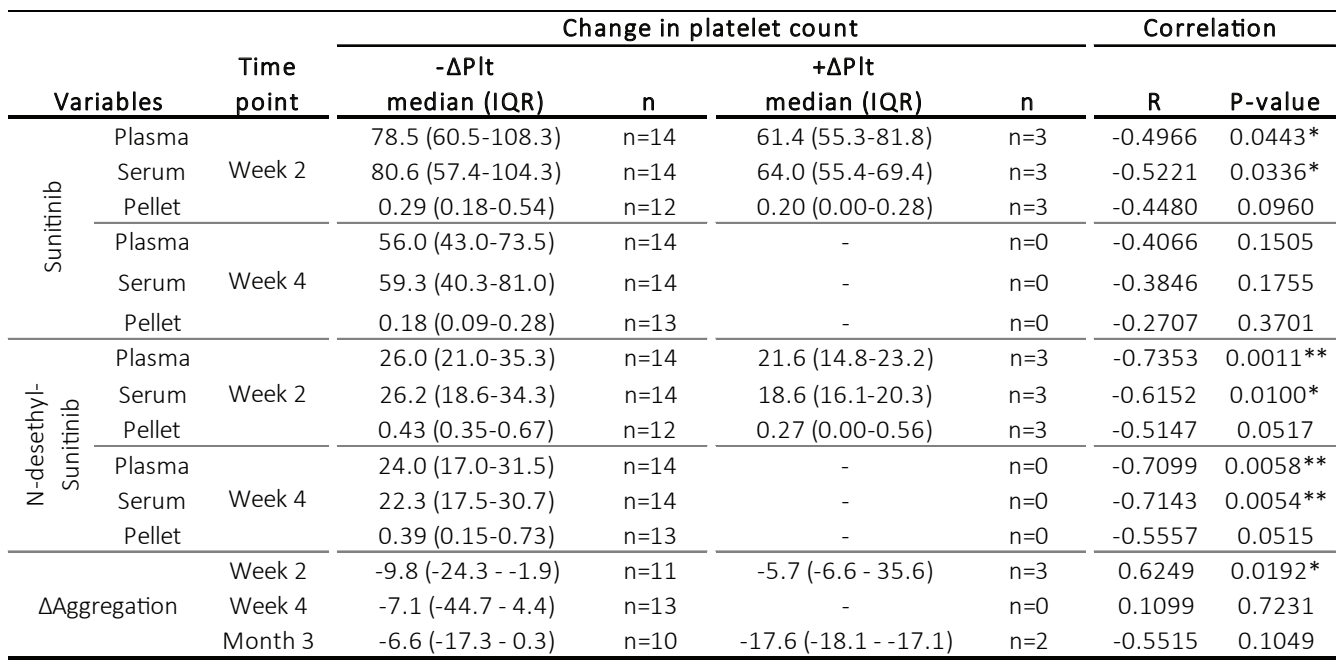

IQR: interquartile range; $\Delta \mathrm{PIt}$ count = Platelet count at indicated timepoint on treatment - platelet count before treatment. Negative value $(-\Delta \mathrm{Plt})$ indicates a reduction in platelet count; Positive value $(+\Delta \mathrm{Plt})$ indicates an increase in platelet count. Correlations are based on all changes in platelet count.
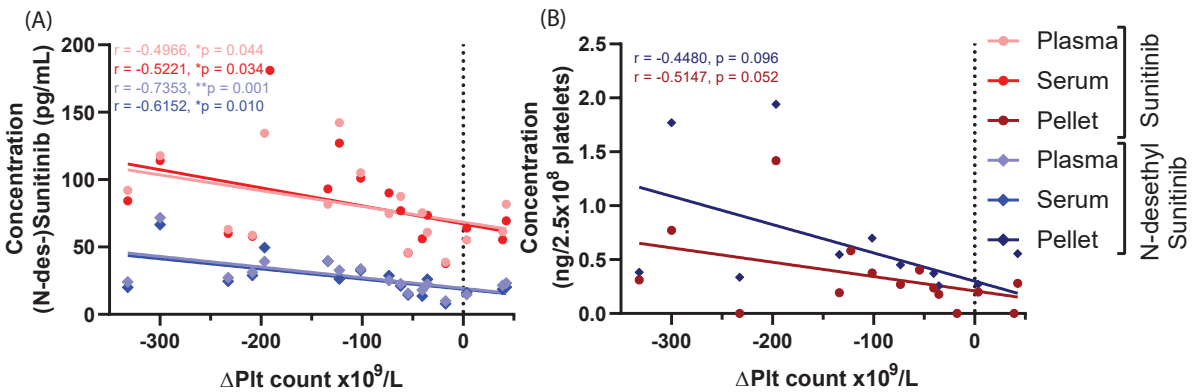

Figure 5.3. Concentrations of ( $\mathrm{N}$-desethyl-)sunitinib correlate with the reduction in platelet count. (A) Correlations between the concentration of sunitinib and $\mathrm{N}$-desethyl-sunitinib in plasma and serum and difference in platelet count $(\triangle \mathrm{Plt}$ count = Plt count at 2 weeks on treatment - platelet count before treatment). (B) Correlation between the concentration of sunitinib and $\mathrm{N}$-desethyl-sunitinib in isolated platelets (normalized to $\mathrm{ng} / 2.5 \times 10^{8}$ platelets) and difference in platelet count. 


\section{Comparative changes in quantitative and qualitative platelet traits by sunitinib treatment of $m R C C$ patients}

For adequate dosing, the collagen concentration required for maximal platelet aggregation was determined using washed patient platelets before the sunitinib treatment. Either 1 or $5 \mu \mathrm{g} /$ $\mathrm{mL}$ collagen was used, dependent on intake of aspirin or atorvastatin (see Table 5.1). This dose was maintained for all follow-up measurements per patient. Platelet aggregation responses on treatment were compared to pre-treatment measurements for each patient. This resulted in an overall decrease in aggregation (range: 5-45\% decrease) in $10 \mathrm{mRCC}$ patients after 2 weeks sunitinib treatment (Figure 5.4A), despite of the observed variation between patients. Platelet aggregation was continued to be decreased in 10 patients (in 7 patients $>10 \%$ ) after 4 weeks, while in 5 patients there was no decrease or even increased aggregation as compared to pre-treatment. Altogether, on average no differences in aggregation were observed after 4 weeks. After 3 months, again no differences in aggregation were observed, due to substantial variation. Remarkably, platelets from 6 patients showed a long-term reduction ( $>10 \%$ decrease) in collagen-induced aggregation until 3 months, while platelets from other patients were not or only minimally affected during this time-period (Figure 5.4A). The five mRCC patients who were treated with both sunitinib and aspirin and/or atorvastatin, revealed no significant differences in platelet aggregation, as compared to patients on sunitinib monotherapy.

We investigated whether an observed reduction in platelet aggregation was correlated to a reduction in platelet count at all time points (Table 5.2). It appeared that the difference in platelet count $(\Delta \mathrm{PIt}=$ Platelet count at 2 weeks on treatment - platelet count before treatment) correlated with the difference in aggregation ( $\triangle \mathrm{P}$ It aggregation = maximum amplitude at 2 weeks on treatment- maximum amplitude before treatment) at two weeks on treatment only (Table 5.2, Figure 5.4B). Next, we compared the reduction in platelet aggregation with the inhibitor levels in plasma, serum and platelets, though no correlation was observed between these parameters (Figure 5.4C).

To further unravel the relationship between the quantitative and qualitative platelet traits and sunitinib levels in patients, we performed a principal component analysis (PCA). The resulting heatmap (Figure 5.4D) indicates which parameters tended to cluster together per component. Four components were uncovered, which combined accounted for $95 \%$ of the variance of this data. It is observed that the parameters contributing to component 1 (C1) included aggregation, count and platelet concentration of ( $\mathrm{N}$-desethyl-)sunitinib at 2 and 4 weeks. $\mathrm{C} 2$ was composed of the parameters of plasma, serum and platelet levels of ( $\mathrm{N}$-desethyl-)sunitinib, and C3 showed the related count levels and metabolite concentrations in plasma and serum at 2 and 4 weeks. C4 revealed an association of aggregation and sunitinib levels in plasma and serum at 4 weeks. Altogether, this PCA underlined that the (N-desethyl-)sunitinib levels in either serum or plasma from $\mathrm{mRCC}$ patients associated with the reduction in both platelet count and aggregation, 
(A)

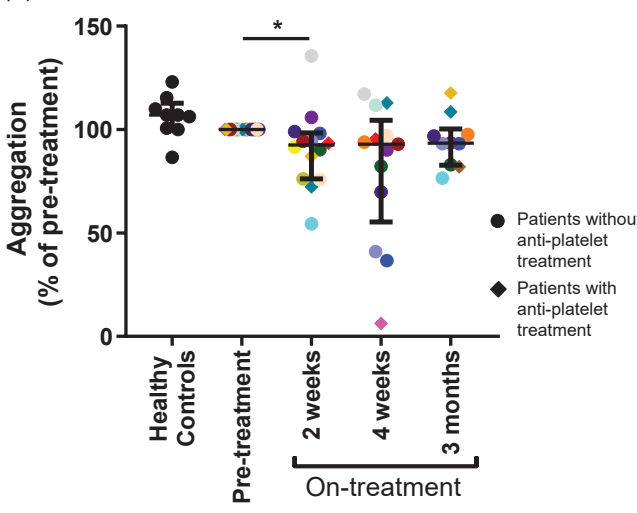

(B)

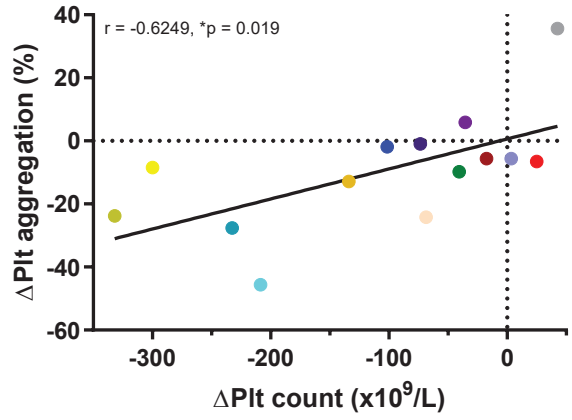

(C)

Count
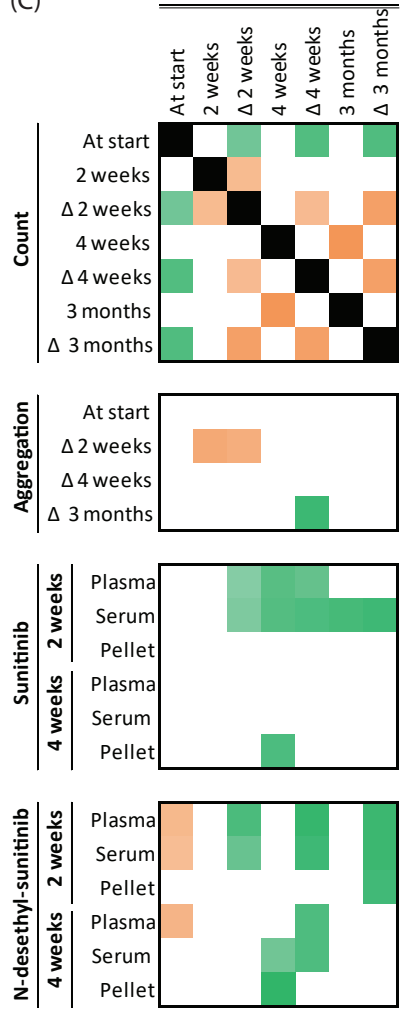
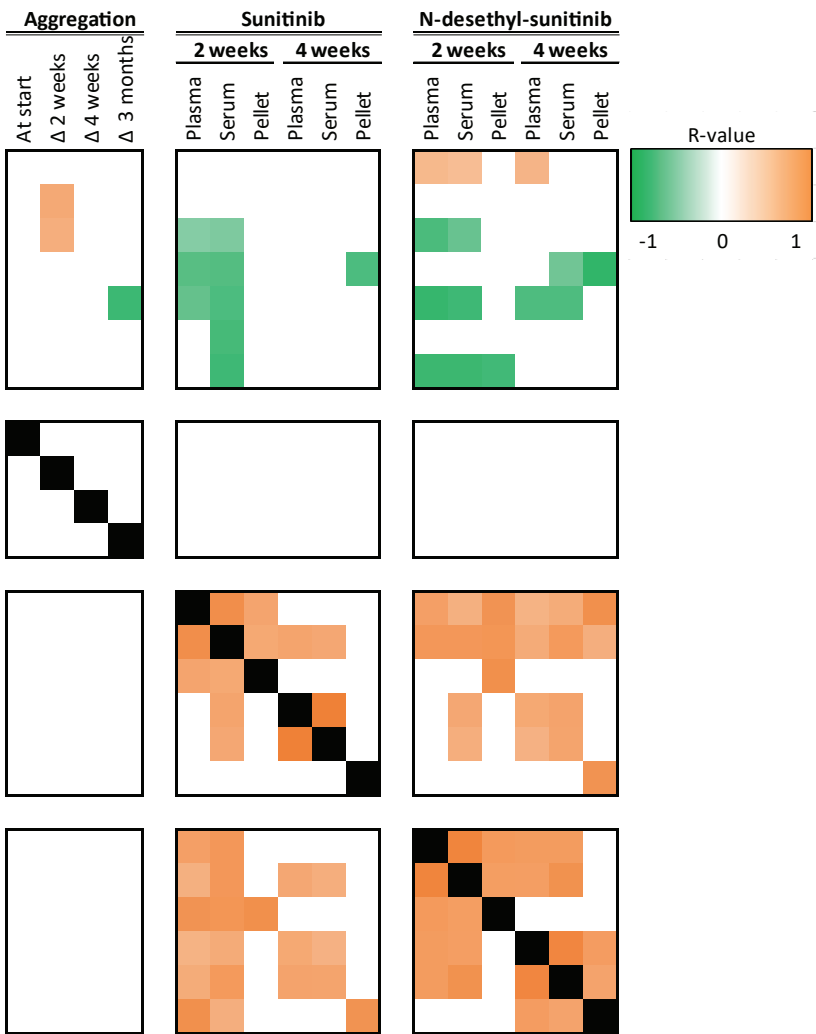

Figure 5.4. Platelet aggregation is reduced in RCC patients after 2 weeks of sunitinib treatment and correlates with reduction in platelet count. Blood was collected from 10 healthy controls and 20 RCC patients the day before, after 2 weeks, 4 weeks and 3 months on sunitinib treatment. (A) Light transmission aggregometry was induced in isolated platelets $\left(250 \times 10^{9} / \mathrm{L}\right)$ by collagen type I. Scatterplots indicate percentage of aggregation normalized to aggregation pretreatment. Circles indicate platelets from patients treated with sunitinib, stimulated with $1 \mu \mathrm{g} / \mathrm{mL}$ collagen; diamonds indicate platelets from patients treated with sunitinib and anti-platelet drugs, stimulated with $5 \mu \mathrm{g} / \mathrm{mL}$ collagen. Individual dots in the scatterplots represent one patient, for color coding see Table 5.1. Lines and error bars represent median \pm interquartile range $(n=10-20), * P<0.05$. (B) Correlation between difference in platelet count $(\Delta P$ It count $=$ Plt count at 2 weeks on treatment - platelet count before treatment) and difference in platelet aggregation $(\Delta \mathrm{Plt}$ aggregation $=$ Maximum amplitude at 2 weeks on treatment - maximum amplitude before treatment). Individual dots in the scatterplots represent one patient, for color coding see Table 5.1. (C) Correlation of significant R values for (changes in) platelet count, change in aggregation response and ( $\mathrm{N}$-desethyl-)sunitnib concentration parameters, with a highly negative correlation in green, a highly positive correlation in orange and no correlation in white. 
suggesting that the quantitative and qualitative platelet traits were jointly affected by the relative exposure to this TKI.

\section{Changes in quantitative and qualitative platelet traits by sunitinib treatment of mRCC patients in relation to reported bleeding and disease progression}

Of the 20 mRCC patients treated with sunitinib, 9 reported bleeding complications during the 3-month follow-up. Events included minor nose bleeds (epistaxis) or bleeding gums (gingivitis), blood in urine (haematuria), stool (gastrointestinal bleeding) or mucus (haemoptysis) (Table 5.1).

(D)

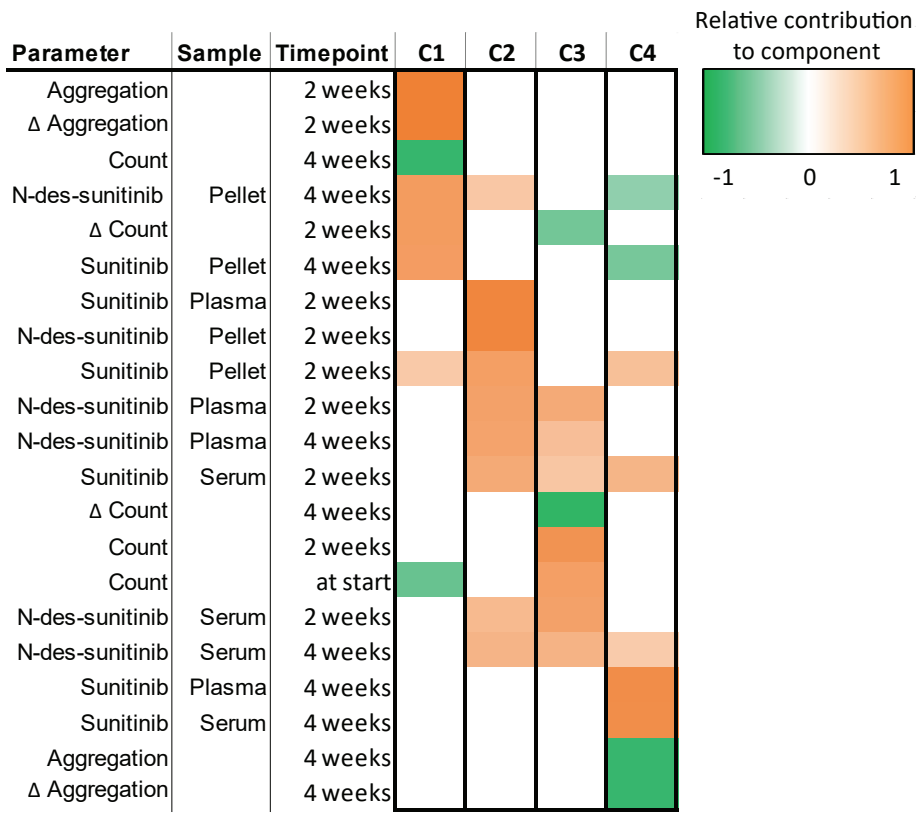

Figure 5.4. Continued. (D) Rotated component matrix determined by principal component analysis. Heatmap shows relative contribution of parameters to the four components (C1-4) with an eigenvalue over 1 . Heatmap was filtered to only include values greater than 0.4 or less than- 0.4 as important contributors to the determined component. Colors refer to a highly negative contribution in green, a highly positive contribution in orange and no correlation in white.

Table 5.3. Reported bleeding correlates with platelet aggregation in RCC patients at 4 weeks on treatment.

\begin{tabular}{|c|c|c|c|c|c|c|c|}
\hline \multirow[b]{3}{*}{ Variables } & \multirow{3}{*}{$\begin{array}{l}\text { Time } \\
\text { point }\end{array}$} & \multicolumn{4}{|c|}{ Reported bleeding } & \multicolumn{2}{|c|}{ Correlation } \\
\hline & & No & & Yes & & & \\
\hline & & median (IQR) & $n$ & median (IQR) & $\mathrm{n}$ & $\mathrm{R}$ & P-value \\
\hline \multirow{3}{*}{ Platelet count } & Week 2 & $132.5(75.8-229.8)$ & $n=9$ & $164.0(119.0-194.8)$ & $n=8$ & -0.0515 & 0.8498 \\
\hline & Week 4 & 78.5 (49.0-202.0) & $n=7$ & $132.3(98.4-155.0)$ & $n=8$ & 0.0201 & 0.9508 \\
\hline & Month 3 & $162.5(104.5-255.8)$ & $n=4$ & $161.5(137.8-202.0)$ & $n=5$ & -0.3651 & 0.3786 \\
\hline \multirow{3}{*}{$\Delta$ Aggregation } & Week 2 & $-10.7(-29.6-4.0)$ & $n=6$ & $-3.8(-6.7-0.8)$ & $n=6$ & 0.2612 & 0.4072 \\
\hline & Week 4 & $-3.8(-5.7-12.2)$ & $n=4$ & $-24.0(-62.3--7.8)$ & $n=8$ & -0.6680 & $0.0206^{*}$ \\
\hline & Month 3 & $-10.2(-22.1-12.7)$ & $n=4$ & $-6.8(-12.0--4.8)$ & $n=5$ & 0.02623 & 0.9516 \\
\hline Progression \% & - & $10.0(0.0-31.3)$ & $n=4$ & $0(-22.3-14)$ & $n=8$ & -0.2500 & 0.4285 \\
\hline
\end{tabular}

IQR: interquartile range. 


\section{Chapter 5}

Interestingly, of the 5 patients who were on dual treatment of sunitinib and aspirin, only 1 patient (also treated with heparin) reported bleeding events during the first cycle of sunitinib treatment.

We analysed whether the altered platelet count correlated with the reported bleeding. As shown in Table 5.3, this was not the case. On the other hand, we observed that platelet function, as measured by collagen-induced aggregation, was negatively correlated with bleeding at 4 weeks on-treatment (Table 5.3), although the number of patients included in this analysis was relatively small $(n=4-8)$. At the other time points this correlation was not observed.

After three months on treatment, CT scans were evaluated for cancer response in 15 patients (Table 5.1). In 5 of these patients progression of the disease was established, with an average of $23 \%$ increase in tumor size. In the other 10 patients no progression was observed (Table 5.1). In this limited group of patients, no correlation was observed between disease progression and reduction in blood cell counts or platelet aggregation (Suppl. Table 5.1).

\section{Discussion}

In the present study with patients treated with sunitinib for $\mathrm{MRCC}$, we observed that changes in platelet count were related to the inhibitor levels in plasma and serum. The reduction in platelet count may thus reflect the effective sunitinib levels in the patient, which may be used for therapeutic drug monitoring ${ }^{19}$. Furthermore, we observed a strong correlation between effects on platelet count and collagen-induced platelet aggregation, regardless of the treatment time point. This pointed to a common effect of this TKI on quantitative and qualitative platelet traits, which was confirmed by PCA. Next to RCC, sunitinib is approved for the treatment of gastrointestinal stromal tumor (GIST) and pancreatic neuroendocrine tumors. Furthermore, phase 3 clinical trials with sunitinib have been completed with positive outcome for non-small cell lung cancer ${ }^{20,21}$. Hence, the results of the present study may also be applicable to other cancer types. In addition, platelet traits have been reported to be affected by other TKIs used for treatment of a large variety of malignancies ${ }^{1,9}$. Also, other anti-cancer drugs can be taken up by platelets ${ }^{22}$. Therefore, the results of the present study with sunitinib may serve as a proof-of-concept with regards to platelet trait effects of other drugs.

We observed that the patients on-treatment who showed a stronger inhibition in platelet aggregation (at normalized platelet count), also displayed a lower platelet count in whole blood. This can be explained by two separate mechanisms elicited by sunitinib, being (i) an effect on megakaryocyte level, culminating in a lower platelet count, and (ii) an effect on platelet function (aggregation), likely via an impaired tyrosine kinase-mediated signaling. Regarding the first mechanism, sunitinib may affect megakaryocytes or precursor cells directly in the bone marrow via interference in the proliferation of megakaryocytes and the production of proplatelets, which processes are dependent on tyrosine kinase activity ${ }^{23}$. Alternatively, the effects on megakaryocytes may be indirect via altered thrombopoietin production. Tumors can stimulate 


\section{Changes in platelet traits and circulating levels of sunitinib-treated patients}

platelet production via de secretion of various cytokines, especially interleukin-6, which can in turn enhance thrombopoietin expression in the liver ${ }^{24}$. A direct effect of sunitinib on tumor proliferation may result in reduced levels of thrombopoietin and thereby lowering of the platelet count. Besides platelet count, we confirmed that sunitinib also reduced the WBC count (up to grade 2 ) in the mRCC patients. Furthermore, also the RBC count, and hemoglobin and hematocrit levels were affected upon sunitinib treatment. These results point to an effect of sunitinib on different hematopoietic cell populations in the bone marrow. Other studies with larger patient numbers indeed concluded that a reduced neutrophil count could be a predictor of PFS and OS probability ${ }^{13,25}$. With regard to RBCs, sunitinib has been reported to affect erythropoiesis in both directions, resulting in either anemia ${ }^{26}$ or in erythrocytosis ${ }^{27}$. Markedly, in our small patient group, we observed a transient increase in RBC count, hemoglobin and hematocrit during the first cycle of sunitinib treatment, as reported previously and explained by cycling kinetics during the dosing schedule ${ }^{28}$.

Regarding the second mechanism of sunitinib affecting tyrosine kinase-mediated platelet signaling, we observed that collagen-induced platelet aggregation was reduced in mRCC patients after 14 days of sunitinib treatment. Others have shown that this inhibition was already present after 24 hours of sunitinib treatment ${ }^{29}$. Besides aggregation, sunitinib also inhibits collageninduced thrombus formation, exposure of phosphatidylserine under flow, as well as $\alpha$-granule secretion $^{11,29}$. As discussed before, the protein tyrosine kinases that are affected by sunitinib are c-Src ${ }^{29}$, as well as other kinases expressed in platelets (e.g., Axl, CSF and Itk) ${ }^{1}$.

As platelets are able to take up sunitinib ${ }^{11}$, we hypothesized that this affects the outcome of sunitinib plasma measurements. Therefore, we compared the concentrations of sunitinib and $\mathrm{N}$-desethyl-sunitinib (active metabolite of sunitinib) in plasma, serum and isolated platelets from mRCC patients at 2 and 4 weeks of treatment. The concentration of N-desethyl-sunitinib was much lower than sunitinib in both serum and plasma, which is in line with previous results ${ }^{30}$. Furthermore, there was no difference between serum and plasma levels of either sunitinib or $\mathrm{N}$-desethyl-sunitinib at 2 or 4 weeks of sunitinib administration. Honeywell et al. also reported no differences for sunitinib in plasma as compared to serum for 5 patients on sunitinib treatment after 24 hours and 3 weeks ${ }^{31}$. In contrast, another study demonstrated lower concentrations of sunitinib in plasma as compared to serum in patients treated with sunitinib ${ }^{29}$. These studies demonstrated a high variation in sunitinib plasma concentrations, which could only partly be explained by patient- or medication-related factors ${ }^{32}$. This highlights the importance for further research of therapeutic drug monitoring for individual dosage adjustments, especially in cases of toxicity ${ }^{19}$. Interestingly, the concentrations of the active metabolite were significantly higher in isolated platelets from mRCC patients at 2 and 4 weeks as compared to that of sunitinib itself. The only difference between the two molecules is an ethyl $(\mathrm{CH} 2-\mathrm{CH} 3)$ group that is removed from sunitinib by cytochrome $\mathrm{P} 450^{33}$. As this enzyme not present in platelets, these results suggest 


\section{Chapter 5}

preferential uptake of the metabolite by these cells. Overall, we concluded that although the concentration of the active metabolite in platelets was higher, the uptake of sunitinib in platelets did not affect the sunitinib concentration in plasma versus serum. The serum concentration of $\mathrm{N}$-desethyl-sunitinib negatively correlated with platelet count at 4 weeks of treatment, suggesting that higher platelet counts result in a lower concentration of metabolite present in serum. Together with the observation that metabolite concentrations in isolated platelets were higher, this may suggest that platelets do not secrete sunitinib after uptake.

Mild bleeding was reported in 9 of the 20 investigated cancer patients on sunitinib. No correlation existed between the platelet count at any time point on treatment and the occurrence of bleeding during the 3-month study period. This agrees with earlier reports that showed that the platelet count is a poor predictor for bleeding ${ }^{34,35}$. Hence, the reported bleeding events may be explained by an accompanying inhibition of platelet activation by sunitinib. We observed a correlation between platelet aggregation responses and bleeding during sunitinib treatment, meaning that stronger reduction in collagen-induced platelet aggregation was observed in patients that reported bleeding episodes. Of note, only a small number of patients was included in this analysis, and at no other time points a correlation was observed between platelet aggregation and reported bleeding. Also, Walraven et al. did not observe this correlation between bleeding and platelet aggregation after 3 weeks of treatment in 5 patients ${ }^{29}$. As normal platelet functions can be considered to be required for effective control of hemostasis, correlations between bleeding and TKI-induced effects on platelet function should be investigated in larger clinical studies. Of note, in patients treated with sunitinib, also endothelial dysfunction and increased capillary leakage can be induced by treatment with sunitinib ${ }^{36}$, which may also play a role in the increased bleeding tendency. Paradoxically, interfering with endothelial cell integrity can also shift the hemostatic balance in favor of thrombosis, and treatment with sunitinib and other TKIs have also been associated with an increase in arterial thromboembolic events in RCC patients ${ }^{36,37}$.

A correlation between platelet count and disease progression in this study could not be observed due to a relatively small number of patients. However, it has already been demonstrated in large, retrospective patient studies that a decrease in platelet count following sunitinib treatment more likely resulted in a response to therapy and longer OS in $\mathrm{MRCC}^{12}$ and GIST ${ }^{13}$. In the present study we clearly showed that a reduction in platelet count is accompanied by an inhibition of platelet aggregation, which were correlated to the ( $\mathrm{N}$-desethyl-)sunitinib levels in either serum or plasma of mRCC patients (component 1 of the PCA). Altogether this suggested that both quantitative and qualitative platelet traits were linked to the exposure to this TKI.

Renal cancer is a disease of the elderly, with most patients being diagnosed between 65 and $74^{38,39}$. Older cancer patients generally have more comorbidities, especially cardio-vascular disease, and therefore often receive anti-coagulant or anti-platelet drugs. In the present study, five patients were treated with sunitinib in combination with aspirin and/or atorvastatin, both 
affecting platelet functions ${ }^{17,18,40}$. In our tests, this did not result in significant differences in platelet aggregation as compared to patients on sunitinib without comedication. One explanation may be an increased platelet reactivity observed in cancer patients ${ }^{41}$, which compensates for the intake of the anti-platelet drugs. On the other hand, a recent study has shown combined effects of ibrutinib and aspirin on collagen-induced aggregation ${ }^{42}$. Therefore, the effects of dual antiplatelet and TKIs treatments on the inhibition of platelet function should be further investigated.

Our study encounters some limitations. Due to the limited number of patients and some missing values, no (clear) correlations were observed between platelet count and function on the one hand and bleeding and disease progression on the other hand. Also, only one platelet function test could be performed due to the limited amount of blood obtained from the patients. Out of multiple function tests, we opted for light transmission aggregometry as this is still the gold standard for platelet function testing. Due to ethical restrictions, the relation between platelet dysfunction after sunitinib treatment and abnormal (pro)platelet formation from megakaryocytes in the bone marrow could not be assessed. For the TKI dasatinib it has been reported that it promoted megakaryocyte differentiation, while it impaired migration and proplatelet formation ${ }^{43}$. The effects of TKI treatment on megakaryocyte proliferation and function should therefore be investigated in future studies.

In summary, in this study with mRCC patients treated with sunitinib a strong correlation was found between the effects on platelet count and inhibition of aggregation as well as (N-desethyl) sunitinib levels in plasma and serum. The effects on count and function may thus reflect the relative exposure of the patient to sunitinib. This pointed to an association between the effect of this TKI on quantitative and qualitative platelet traits. These results may serve as a proof-ofprinciple with regards to other drugs, indicating that TKI treatment can affect both platelet count and function, which could be used as a measure for relative drug exposure and therapeutic drug monitoring.

\section{Acknowledgements}

This study was supported by Pfizer as an Investigator-Initiated Research grant to MJEK (Tracking Number WI209458).

\section{References}

1. Tullemans BME, Heemskerk JWM, Kuijpers MJE. Acquired platelet antagonism: off-target antiplatelet effects of malignancy treatment with tyrosine kinase inhibitors. J Thromb Haemost. 2018;16:168699.

2. Gross S, Rahal R, Stransky N, Lengauer C, Hoeflich KP. Targeting cancer with kinase inhibitors. J Clin Invest. 2015;125:1780-9.

3. Siegel RL, Miller KD, Jemal A. Cancer statistics, 2020. CA Cancer J Clin. 2020;70:7-30.

4. Motzer RJ, Tannir NM, McDermott DF, Aren Frontera O, Melichar B, Choueiri TK, et al. Nivolumab plus Ipilimumab versus Sunitinib in Advanced Renal-Cell Carcinoma. New Engl J Med. 2018;378:1277-90.

5. Rini BI, Plimack ER, Stus V, Gafanov R, Hawkins R, Nosov D, et al. Pembrolizumab plus Axitinib versus 


\section{Chapter 5}

Sunitinib for Advanced Renal-Cell Carcinoma. New Engl J Med. 2019;380:1116-27.

6. Motzer RJ, Escudier B, McDermott DF, George S, Hammers HJ, Srinivas S, et al. Nivolumab versus Everolimus in Advanced Renal-Cell Carcinoma. New Engl J Med. 2015;373:1803-13.

7. Choueiri TK, Escudier B, Powles T, Tannir NM, Mainwaring PN, Rini BI, et al. Cabozantinib versus everolimus in advanced renal cell carcinoma (METEOR): final results from a randomised, open-label, phase 3 trial. Lancet Oncol. 2016;17:917-27.

8. Versteeg HH, Heemskerk JW, Levi M, Reitsma PH. New fundamentals in hemostasis. Physiol Rev. 2013;93:327-58.

9. Levade M, Severin S, Gratacap MP, Ysebaert L, Payrastre B. Targeting Kinases in Cancer Therapies: Adverse Effects on Blood Platelets. Curr Pharm Des. 2016;22:2315-22.

10. Je Y, Schutz FA, Choueiri TK. Risk of bleeding with vascular endothelial growth factor receptor tyrosine-kinase inhibitors sunitinib and sorafenib: a systematic review and meta-analysis of clinical trials. Lancet Oncol. 2009;10:967-74.

11. Sabrkhany S, Griffioen AW, Pineda S, Sanders L, Mattheij N, van Geffen JP, et al. Sunitinib uptake inhibits platelet function in cancer patients. Eur J Cancer. 2016;66:47-54.

12. Hamilton Z, Lee HJ, Jimenez J, Lane BR, Wang S, Beksac AT, et al. Change in platelet count as a prognostic indicator for response to primary tyrosine kinase inhibitor therapy in metastatic renal cell carcinoma. BJU Int. 2016;118:927-34.

13. Den Hollander D, Van der Graaf WTA, Desar IME, Le Cesne A. Predictive factors for toxicity and survival of second-line sunitinib in advanced gastrointestinal stromal tumours (GIST). Acta Oncol. 2019;58:1648-54.

14. Stanworth SJ, Dyer C, Casbard A, Murphy MF. Feasibility and usefulness of self-assessment of bleeding in patients with haematological malignancies, and the association between platelet count and bleeding. Vox Sang. 2006;91:63-9.

15. van der Meijden PE, Feijge MA, Giesen PL, Huijberts M, van Raak LP, Heemskerk JW. Platelet P2Y12 receptors enhance signalling towards procoagulant activity and thrombin generation. A study with healthy subjects and patients at thrombotic risk. Thromb Haemost. 2005;93:1128-36.

16. de Bruijn P, Sleijfer S, Lam MH, Mathijssen RH, Wiemer EA, Loos WJ. Bioanalytical method for the quantification of sunitinib and its n-desethyl metabolite SU12662 in human plasma by ultra performance liquid chromatography/tandem triple-quadrupole mass spectrometry. J Pharm Biomed Anal. 2010;51:934-41.

17. Zhao L, Liu D, Liu B, Hu H, Cui W. Effects of atorvastatin on ADP-, arachidonic acid-, collagen-, and epinephrine-induced platelet aggregation. J Int Med Res. 2017;45:82-8.

18. Ni R, Peleg T, Gross PL. Atorvastatin delays murine platelet activation in vivo even in the absence of endothelial NO synthase. Arterioscl Thromb Vasc Biol. 2012;32:2609-15.

19. Verheijen RB, Yu H, Schellens JHM, Beijnen JH, Steeghs N, Huitema ADR. Practical Recommendations for Therapeutic Drug Monitoring of Kinase Inhibitors in Oncology. Clin Pharmacol Ther. 2017;102:76576.

20. Scagliotti GV, Krzakowski M, Szczesna A, Strausz J, Makhson A, Reck M, et al. Sunitinib plus erlotinib versus placebo plus erlotinib in patients with previously treated advanced non-small-cell lung cancer: a phase III trial. J Clin Oncol. 2012;30:2070-8.

21. Baggstrom MQ, Socinski MA, Wang XF, Gu L, Stinchcombe TE, Edelman MJ, et al. Maintenance Sunitinib following Initial Platinum-Based Combination Chemotherapy in Advanced-Stage IIIB/IV Non-Small Cell Lung Cancer: A Randomized, Double-Blind, Placebo-Controlled Phase III Study-CALGB 30607 (Alliance). J Thorac Oncol. 2017;12:843-9.

22. Verheul HM, Lolkema MP, Qian DZ, Hilkes YH, Liapi E, Akkerman JW, et al. Platelets take up the monoclonal antibody bevacizumab. Clin Cancer Res. 2007;13:5341-7.

23. Noetzli LJ, French SL, Machlus KR. New Insights Into the Differentiation of Megakaryocytes From Hematopoietic Progenitors. Arterioscl Thromb Vasc Biol. 2019;39:1288-300.

24. Lin RJ, Afshar-Kharghan V, Schafer Al. Paraneoplastic thrombocytosis: the secrets of tumor selfpromotion. Blood. 2014;124:184-7.

25. Rautiola J, Donskov F, Peltola K, Joensuu H, Bono P. Sunitinib-induced hypertension, neutropaenia and thrombocytopaenia as predictors of good prognosis in patients with metastatic renal cell carcinoma. BJU Int. 2016;117:110-7. 
26. Motzer RJ, Rini BI, Bukowski RM, Curti BD, George DJ, Hudes GR, et al. Sunitinib in patients with metastatic renal cell carcinoma. JAMA. 2006;295:2516-24.

27. Alexandrescu DT, McClure R, Farzanmehr H, Dasanu CA. Secondary erythrocytosis produced by the tyrosine kinase inhibitors sunitinib and sorafenib. J Clin Oncol. 2008;26:4047-8.

28. van der Veldt AA, Boven E, Vroling L, Broxterman HJ, van den Eertwegh AJ, Haanen JG. Sunitinibinduced hemoglobin changes are related to the dosing schedule. J Clin Oncol. 2009;27:1339-40; author reply 40-2.

29. Walraven M, Homs MYV, van der Veldt AAM, Dekker H, Koldenhof J, Honeywell R, et al. Platelet function is disturbed by the angiogenesis inhibitors sunitinib and sorafenib, but unaffected by bevacizumab. Angiogenesis. 2018;21:325-34.

30. Houk BE, Bello CL, Poland B, Rosen LS, Demetri GD, Motzer RJ. Relationship between exposure to sunitinib and efficacy and tolerability endpoints in patients with cancer: results of a pharmacokinetic/ pharmacodynamic meta-analysis. Cancer Chemother Pharmacol. 2010;66:357-71.

31. Honeywell R, Yarzadah K, Giovannetti E, Losekoot N, Smit EF, Walraven M, et al. Simple and selective method for the determination of various tyrosine kinase inhibitors used in the clinical setting by liquid chromatography tandem mass spectrometry. J Chromatogr B Analyt Technol Biomed Life Sci. 2010;878:1059-68.

32. Lankheet NA, Knapen LM, Schellens JH, Beijnen JH, Steeghs N, Huitema AD. Plasma concentrations of tyrosine kinase inhibitors imatinib, erlotinib, and sunitinib in routine clinical outpatient cancer care. Ther Drug Monit. 2014;36:326-34.

33. Adams VR, Leggas M. Sunitinib malate for the treatment of metastatic renal cell carcinoma and gastrointestinal stromal tumors. Clin Ther. 2007;29:1338-53.

34. Slichter SJ. Relationship between platelet count and bleeding risk in thrombocytopenic patients. Transfus Med Rev. 2004;18:153-67.

35. Baaten C, Moenen F, Henskens YMC, Swieringa F, Wetzels RJH, van Oerle R, et al. Impaired mitochondrial activity explains platelet dysfunction in thrombocytopenic cancer patients undergoing chemotherapy. Haematologica. 2018;103:1557-67.

36. Bair SM, Choueiri TK, Moslehi J. Cardiovascular complications associated with novel angiogenesis inhibitors: emerging evidence and evolving perspectives. Trends Cardiovasc Med. 2013;23:104-13.

37. Choueiri TK, Schutz FA, Je Y, Rosenberg JE, Bellmunt J. Risk of arterial thromboembolic events with sunitinib and sorafenib: a systematic review and meta-analysis of clinical trials. J Clin Oncol. 2010;28:2280-5.

38. Howlader N, Noone AM, Krapcho M, Miller D, Brest A, Yu M, et al. SEER Cancer Statistics Review, 1975-2017. National Cancer Institute 2019.

39. Padala SA, Barsouk A, Thandra KC, Saginala K, Mohammed A, Vakiti A, et al. Epidemiology of Renal Cell Carcinoma. World J Oncol. 2020;11:79-87.

40. Warner TD, Nylander S, Whatling C. Anti-platelet therapy: cyclo-oxygenase inhibition and the use of aspirin with particular regard to dual anti-platelet therapy. Br J Clin Pharmacol. 2011;72:619-33.

41. Goubran HA, Stakiw J, Radosevic M, Burnouf T. Platelet-cancer interactions. Sem Thromb Hemost. 2014;40:296-305.

42. Ninomoto J, Mokatrin A, Kinoshita T, Marimpietri C, Barrett TD, Chang BY, et al. Effects of ibrutinib on in vitro platelet aggregation in blood samples from healthy donors and donors with platelet dysfunction. Hematology. 2020;25:112-7.

43. Mazharian A, Ghevaert C, Zhang L, Massberg S, Watson SP. Dasatinib enhances megakaryocyte differentiation but inhibits platelet formation. Blood. 2011;117:5198-206. 


\section{Supplemental data}

Supplemental Table 5.1. No correlation of disease progression with changes in blood cell counts and platelet aggregation in $\mathrm{mRCC}$ patients

\begin{tabular}{|c|c|c|c|c|c|c|c|}
\hline \multirow[b]{2}{*}{ Variables } & \multirow[b]{2}{*}{$\begin{array}{l}\text { Time } \\
\text { point }\end{array}$} & \multicolumn{4}{|c|}{ Reported bleeding } & \multicolumn{2}{|c|}{ Correlation } \\
\hline & & $\begin{array}{l}\text { No (PR + SD) } \\
\text { median (IQR) }\end{array}$ & $n$ & $\begin{array}{c}\text { Yes (PD) } \\
\text { median (IQR) } \\
\end{array}$ & $\mathrm{n}$ & $\mathrm{R}$ & P-value \\
\hline \multirow{3}{*}{$\Delta$ Platelet count } & Week 2 & $-87.5(-189.9--36.8)$ & $n=8$ & $-36.5(-60.3-24.8)$ & $n=4$ & 0.4609 & 0.1535 \\
\hline & Week 4 & $-143.3(-194.6--45.1)$ & $\mathrm{n}=8$ & $-73.0(-147.0--8.8)$ & $n=5$ & 0.2535 & 0.4351 \\
\hline & Month 3 & $-77.8(-137.8--8.6)$ & $n=8$ & $4.5(-42.5-51.5)$ & $n=2$ & 0.4352 & 0.2667 \\
\hline \multirow{3}{*}{$\begin{array}{l}\Delta \text { White blood } \\
\text { cell count }\end{array}$} & Week 2 & $-1.68(-4.04--0.08)$ & $n=8$ & $-0.68(-2.05-1.26)$ & $n=4$ & 0.4104 & 0.198 \\
\hline & Week 4 & $-3.65(-5.03--2.02)$ & $n=8$ & $-2.65(-3.18--0.83)$ & $n=5$ & 0.4655 & 0.1181 \\
\hline & Month 3 & $-3.70(-5.70--1.35)$ & $n=8$ & 0.65 & $\mathrm{n}=1$ & 0.5477 & 0.2222 \\
\hline \multirow{3}{*}{$\begin{array}{l}\Delta \text { Red blood } \\
\text { cell count }\end{array}$} & Week 2 & $0.21(0.20-0.33)$ & $n=8$ & $0.25(-0.07-0.41)$ & $n=4$ & 0.1282 & 0.7152 \\
\hline & Week 4 & $-0.06(-0.17-0.18)$ & $\mathrm{n}=8$ & $0.08(-0.01-0.35)$ & $n=5$ & 0.3803 & 0.2222 \\
\hline & Month 3 & $-0.93(-1.73--0.50)$ & $n=8$ & -0.87 & $n=1$ & 0.1369 & 0.8889 \\
\hline \multirow{3}{*}{$\Delta$ Aggregation } & Week 2 & $-7.7(-24.0--1.2)$ & $n=8$ & -5.7 & $n=1$ & 0.0688 & 1.0000 \\
\hline & Week 4 & $-24.0(-60.2--4.2)$ & $n=8$ & $-6.6(-72.1-7.3)$ & $n=4$ & 0.2132 & 0.6095 \\
\hline & Month 3 & $-6.6(-14.5-5.6)$ & $n=6$ & $-10.2(-18.1--2.4)$ & $n=2$ & -0.0870 & 0.8889 \\
\hline
\end{tabular}

IQR: interquartile range; PR: partial response; PD: progressive disease; SD: stable disease. 
5 

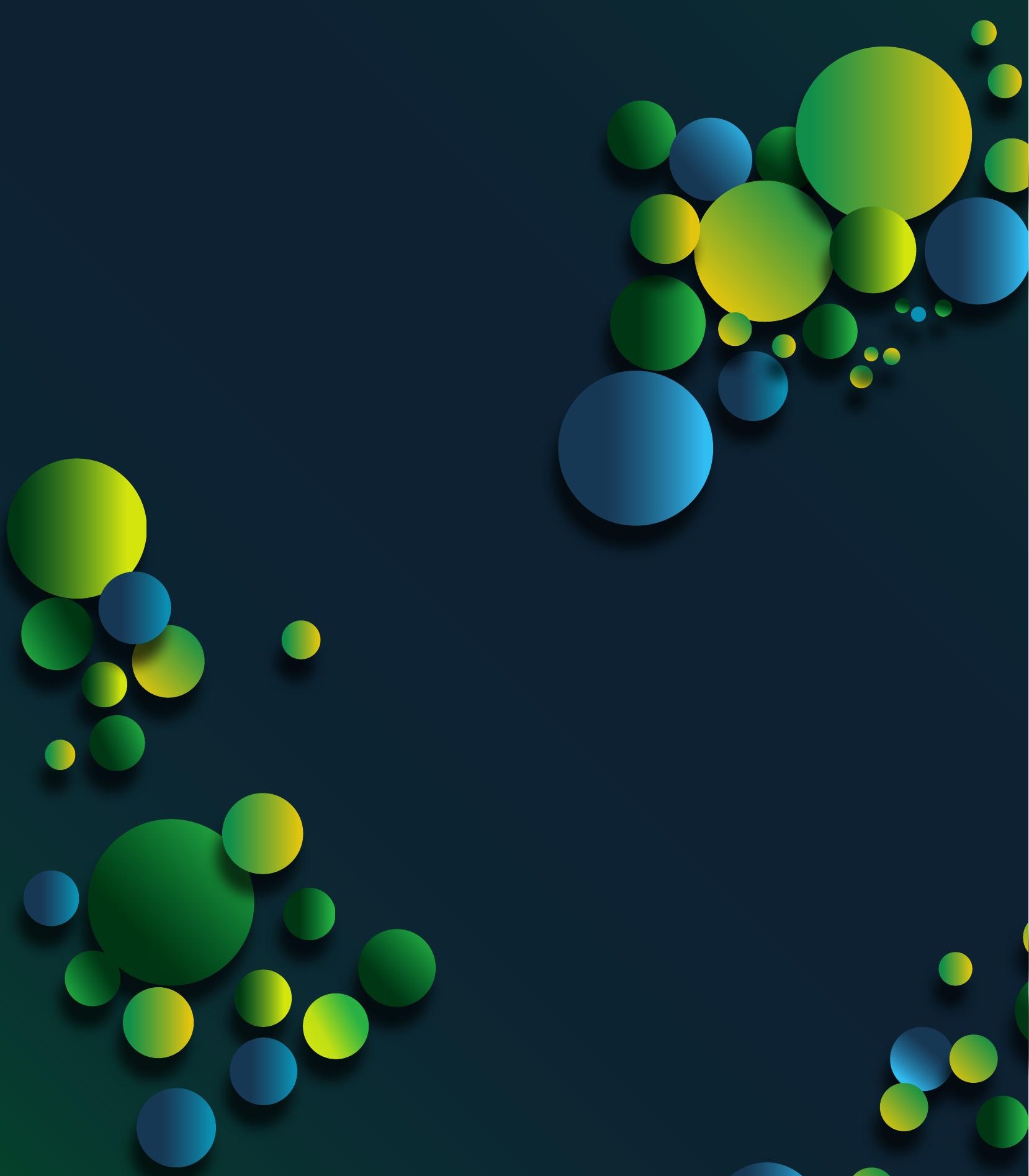


\section{Chapter 6}

Comparison of inhibitory effects of irreversible and reversible Btk inhibitors on platelet function

Tullemans BME, Karel MFA, Léopold V, ten Brink MS, Baaten CCFMJ, Maas SL, de Vos AF, Eble JA, Nijziel MR, van der Vorst EPC, Cosemans JMEM, Heemskerk JWM, Claushuis TAM* and Kuijpers MJE* (* equal contribution)

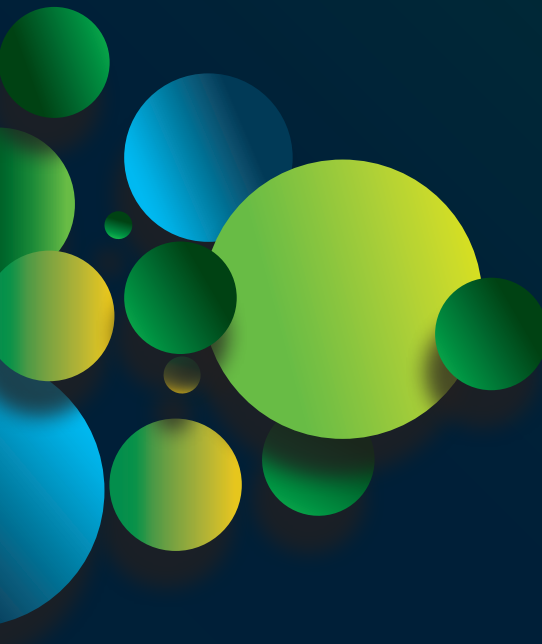

Brit J Haematol 2021; 1-15 Reprinted with permission 


\section{Abstract}

All irreversible Bruton tyrosine kinase (Btk) inhibitors, including ibrutinib and acalabrutinib induce platelet dysfunction and increased bleeding risk. New reversible Btk inhibitors were developed, like MK-1026. The mechanism underlying increased bleeding tendency with Btk inhibitors remains unclear. We investigated the effects of ibrutinib, acalabrutinib and MK-1026 on platelet function in healthy volunteers, patients and Btk-deficient mice, together with offtarget effects on tyrosine kinase phosphorylation. All inhibitors suppressed GPVI- and CLEC-2mediated platelet aggregation, activation and secretion in a dose-dependent manner. Only ibrutinib inhibited thrombus formation on vWF-co-coated surfaces, while on collagen this was not affected. In blood from Btk-deficient mice, collagen-induced thrombus formation under flow was reduced, but preincubation with either inhibitor was without additional effects. MK-1026 showed less off-target effects upon GPVI-induced TK phosphorylation as compared to ibrutinib and acalabrutinib. In ibrutinib-treated patients, GPVI-stimulated platelet activation and adhesion on vWF-Co-coated surfaces were inhibited, while CLEC-2 stimulation induced variable responses. The dual inhibition of GPVI and CLEC-2 signalling by Btk inhibitors might account for the increased bleeding tendency, with ibrutinib causing more high-grade bleedings due to additional inhibition of platelet-vWF interaction. As MK-1026 showed less off-target effects and only affected activation of isolated platelets, it might be promising for future treatment. 


\section{Introduction}

Ibrutinib, an irreversible Bruton's tyrosine kinase (Btk) inhibitor, is widely used to treat multiple haematological malignancies ${ }^{1-5}$. Ibrutinib treatment shows high response rates and improved progression-free survival, with decreased toxicity when compared to conventional treatment options ${ }^{1-6}$. However, ibrutinib is prescribed life-long (or until progression) and requires side effect management. These toxicities are responsible for discontinuation in up to $20 \%$ of patients $^{6-9}$. A prominent side effect of ibrutinib is bleeding, with over $50 \%$ of patients experiencing bleeding events within 3 years, ranging from mild mucocutaneous bleeding to life-threatening haemorrhage $\mathrm{e}^{3,6,8,10}$. Studies have suggested that ibrutinib-associated bleeding tendency is caused by interference with platelet GPVI signalling pathway through off-target effects on Src kinases ${ }^{11,12}$.

Recently, multiple other Btk inhibitors have been developed, including the irreversible Btk inhibitor acalabrutinib and the reversible Btk inhibitor MK-1026 (formerly known as ARQ$531)^{13,14}$. Opposed to data suggesting that acalabrutinib shows less off-target effects, it still causes an increased bleeding tendency in patients, albeit showing mainly mild bleeding ${ }^{15}$. Studies investigating GPVI inhibitors as potential antithrombotic drugs have shown that inhibition on receptor level does not impair haemostasis ${ }^{16}$. Also, GPVI-deficient patients exhibit only a mild bleeding diathesis ${ }^{17}$ and many potential patients are undiagnosed as they remain asymptomatic ${ }^{18}$. In agreement, studies in mice have shown that GPVI deficiency alone does not impair haemostasis ${ }^{19-21}$, while combined GPVI and CLEC-2 deficiency does ${ }^{21}$. Furthermore, Btk was shown to be involved in GPIb-vWF mediated platelet adhesion, as well as integrin $\alpha_{1 \mid \mathrm{b}} \beta_{3}$ outside-in activation which were inhibited by ibrutinib ${ }^{6,22,23}$. Therefore, the current mechanism of increased bleeding tendency in patients using Btk inhibitors is still unclear and underlines the importance for treatment decisions and the development of novel Btk inhibitors. We therefore investigated the effect of multiple Btk inhibitors on platelet function pathways in healthy volunteers, patients and Btk knock-out (KO) mice.

\section{Materials and Methods}

Materials and methods are available in Supplemental Data.

\section{Results}

Ibrutinib and acalabrutinib inhibit platelet activation and aggregation by GPVI and CLEC-2 in a dose-dependent manner

Treatment with ibrutinib, but also acalabrutinib, can increase bleeding tendency, ${ }^{6}$. To assess the underlying pathways, we investigated platelet activation mediated by multiple agonists in presence of ibrutinib or acalabrutinib.

Both ibrutinib and acalabrutinib inhibited aggregation of washed platelets and plateletrich plasma induced by $1 \mathrm{\mu g} / \mathrm{ml}$ collagen in a dose-dependent manner (Fig 6.1A). In isolated 
platelets, a significant inhibition was observed at $1 \mu \mathrm{M}$ and higher for both compounds (Fig 6.1Ai). In the presence of plasma, a higher concentration of 5-10 $\mu \mathrm{M}$ was required to significantly inhibit collagen-induced platelet aggregation (Fig 6.1Aii). The $I_{50}$ values for ibrutinib and acalabrutinib were 7.7 and 6.0 times lower in washed platelets as compared to PRP (Suppl Table 6.1). At a higher collagen concentration, aggregation was not affected by either inhibitor. (Suppl Fig 6.1Aii). CLEC2-induced platelet aggregation was also inhibited by both acalabrutinib and ibrutinib in a similar dose-dependent manner (Fig 6.1B). GPVI-induced integrin $\alpha_{\| 1 b} \beta_{3}$ activation, P-selectin expression, and PS-exposure were also dose-dependently reduced by both inhibitors, although lower

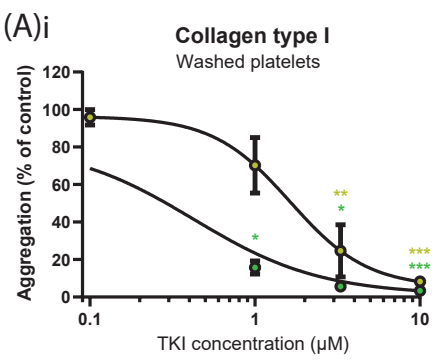

(C) $\mathrm{i}$

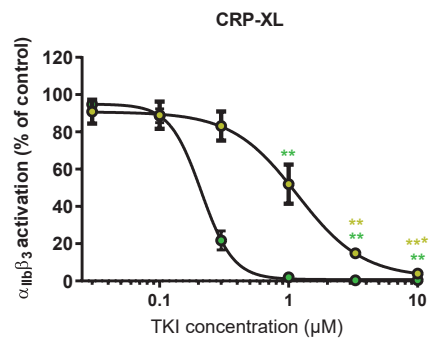

(E) i

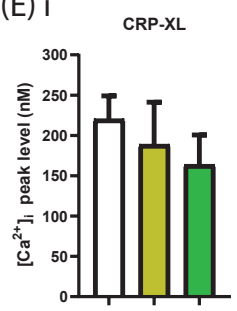

ii ii

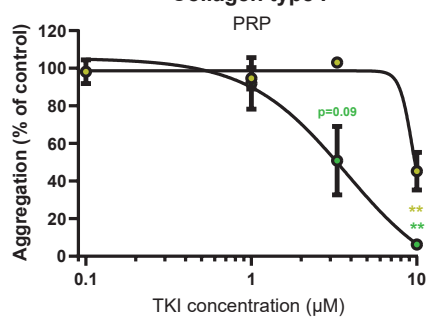

ii

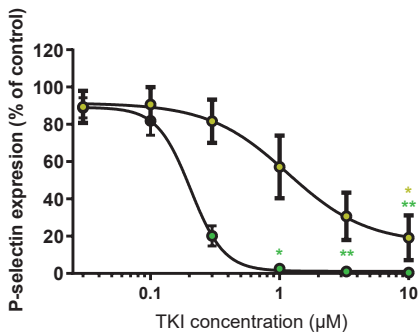

(B)

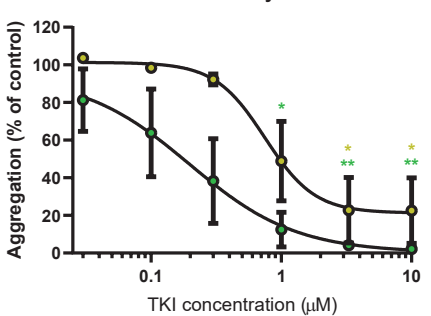

(D)

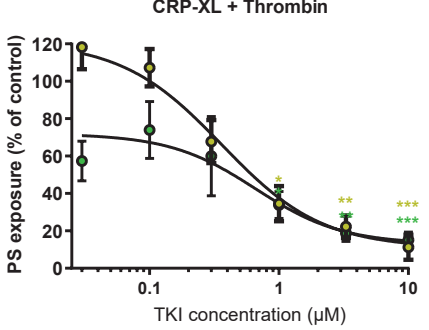

-o- Acalabrutinib

-o- Ibrutinib

Figure 6.1. Ibrutinib and acalabrutinib inhibit GPVI- and CLEC-2-induced platelet activation and aggregation in a dose-dependent manner. Washed platelets (Ai, B) or PRP (Aii) $\left(250 \times 10^{9} / \mathrm{L}\right)$ from healthy volunteers were incubated with different concentrations of acalabrutinib or ibrutinib for 10 minutes at $37^{\circ} \mathrm{C}$. Shown are dose-response curves of aggregation induced by collagen type I ( $\mathrm{A}, 1 \mu \mathrm{g} / \mathrm{ml}$ ) or rhodocytin $(\mathrm{B}, 1 \mu \mathrm{g} / \mathrm{ml})$ in presence of acalabrutinib (lime circles) or ibrutinib (green circles) normalised to control. (C) Washed platelets $\left(100 \times 10^{9} / \mathrm{L}\right)$ were pre-incubated with different concentrations of acalabrutinib or ibrutinib and stimulated with collagen-related peptide (CRP-XL, $5 \mu \mathrm{g} / \mathrm{mL}$ ) for 15 minutes and simultaneously stained for activated integrin $\alpha_{\| b} \beta_{3}$ (i) and $\alpha$-granule secretion (P-selectin, ii). Dose response curves show the percentages of platelets binding FITC-labelled PAC-1 monoclonal antibody or AF647-labelled anti-human CD62 mAb normalized to control expression. (D) Platelets pre-treated with different concentrations of acalabrutinib or ibrutinib were stimulated using both CRP-XL $(5 \mu \mathrm{g} / \mathrm{mL})$ and thrombin $(4 \mathrm{nM})$ for 60 minutes at $37^{\circ} \mathrm{C}$ and PS-exposure was determined by flow cytometry. Histogram shows percentage of platelets binding FITC-labelled annexin A5 normalized to control expression. (E) Fura-2-loaded platelets $\left(200 \times 10^{9} / \mathrm{L}\right)$ pre-incubated with vehicle, acalabrutinib (1 $\left.\mu \mathrm{M}\right)$ or ibrutinib (1 $\mu \mathrm{M})$ were stimulated with $\mathrm{CRP}-\mathrm{XL}(10 \mu \mathrm{g} / \mathrm{mL})$ in presence of $2 \mathrm{mM} \mathrm{CaCl}$. Histograms show (i) maximal rise in $\left[\mathrm{Ca}^{2+}\right]_{\mathrm{i}}$ and (ii) slope. Data are shown as means \pm SEM ( $n=6-7) .{ }^{*} p<0.05, * * p<0.01$ vs control (vehicle). 
concentrations of ibrutinib $(<1 \mu \mathrm{M})$ resulted in significant inhibition as compared to acalabrutinib (Fig 6.1C-D). The $I C_{50}$ values of ibrutinib for the inhibition of integrin $\alpha_{11 b} \beta_{3}$ activation, P-selectin expression and PS exposure were 5.0, 8.2 and 2.2 times lower, respectively, than for acalabrutinib (Suppl. Table 6.1). Furthermore, both compounds did not alter the peak level of intracellular $\mathrm{Ca}^{2+}$ elevations, but the slope of this response was reduced in ibrutinib-treated platelets (Fig 6.1E).

Aggregation induced by the $P 2 Y_{12}$ agonist 2MeS-ADP, the thromboxane $A_{2}$ analogue U46619, and thrombin (Suppl Fig 6.1A) as well as ristocetin remained unaffected in presence of Btk inhibitors (Suppl Fig 6.1B). ADP-stimulated $\alpha_{\| b} \beta_{3}$ activation and $\alpha$-granule secretion were mildly inhibited by both Btk inhibitors (Suppl Fig 6.1C). No effects on $\alpha_{\| 1 b} \beta_{3}$ activation or $\alpha$-granule secretion were observed upon thrombin activation (Suppl Fig 6.1C). Altogether, these results suggest that ibrutinib and acalabrutinib inhibit both GPVI- and CLEC-2-mediated platelet aggregation, albeit lower concentrations of ibrutinib were required. This might explain the increased bleeding tendency seen with both inhibitors.

\section{Ibrutinib, but not acalabrutinib, impairs thrombus formation in whole blood on surfaces co-coated with vWF}

We for the first time investigated the effects of ibrutinib and acalabrutinib on whole blood thrombus formation under flow, simultaneously over multiple surfaces ${ }^{24}$. This allowed systematic analysis of platelet activation and thrombus formation on collagen type I and III and VWF co-coated with laminin, rhodocytin, ristocetin or fibrinogen. In the presence of plasma, concentrations higher than $3.3 \mu \mathrm{M}$ were required to significantly inhibit collagen-induced aggregation (Fig 6.1A). Considering previous in vitro studies, as well as plasma concentrations of ibrutinib and acalabrutinib in patients for inhibition of B-cell carcinomas ${ }^{15,25}$, we selected $1 \mu \mathrm{M}$ to inhibit washed platelets and $5 \mu \mathrm{M}$ to inhibit platelets in the presence of plasma.

Microscopic visualization of platelet adhesion, activation and thrombus formation on collagen showed that neither of the two inhibitors affected these processes, except for reduced PS-exposure by ibrutinib (Fig 6.2A). This was confirmed by image analysis resulting in five thrombus parameters (P1-P5) and three activation markers (P6-8, Suppl Fig 6.2). To systematically summarize the effects on all parameters, cumulative histograms were generated showing that ibrutinib, but not acalabrutinib, reduced PS-exposure on collagen type I and III (Fig 2B, P8). Upon blood perfusion over surfaces that trigger GPIb alone (vWF+ristocetin) or in combination with CLEC-2 (vWF+rhodocytin), $\alpha_{6} \beta_{1}$ (vWF+laminin) or $\alpha_{\| 1} \beta_{3}$ (vWF+fibrinogen), only ibrutinib decreased almost all parameters of thrombus formation (Fig 6.2B, Suppl Fig 6.2). In comparison to control, thrombi were smaller and less compact in structure with ibrutinib and showed reduced expression of activated integrin $\alpha_{11 b} \beta_{3}$ and P-selectin ( $\alpha$-granule release) (Suppl Fig 6.2). These results show that, in contrast to acalabrutinib, platelet adhesion to surfaces co-coated with VWF was strongly impaired by ibrutinib. 
(A)

Collagen type I
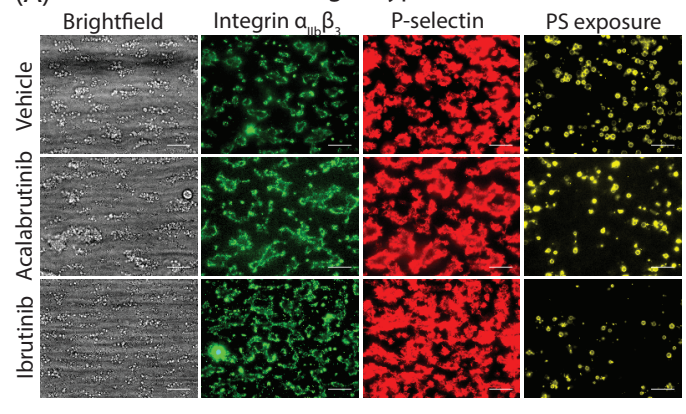

Brightfield

$\mathrm{VWF}+$ rhodocytin

(B)i Collagen type I

ii Collagen type III
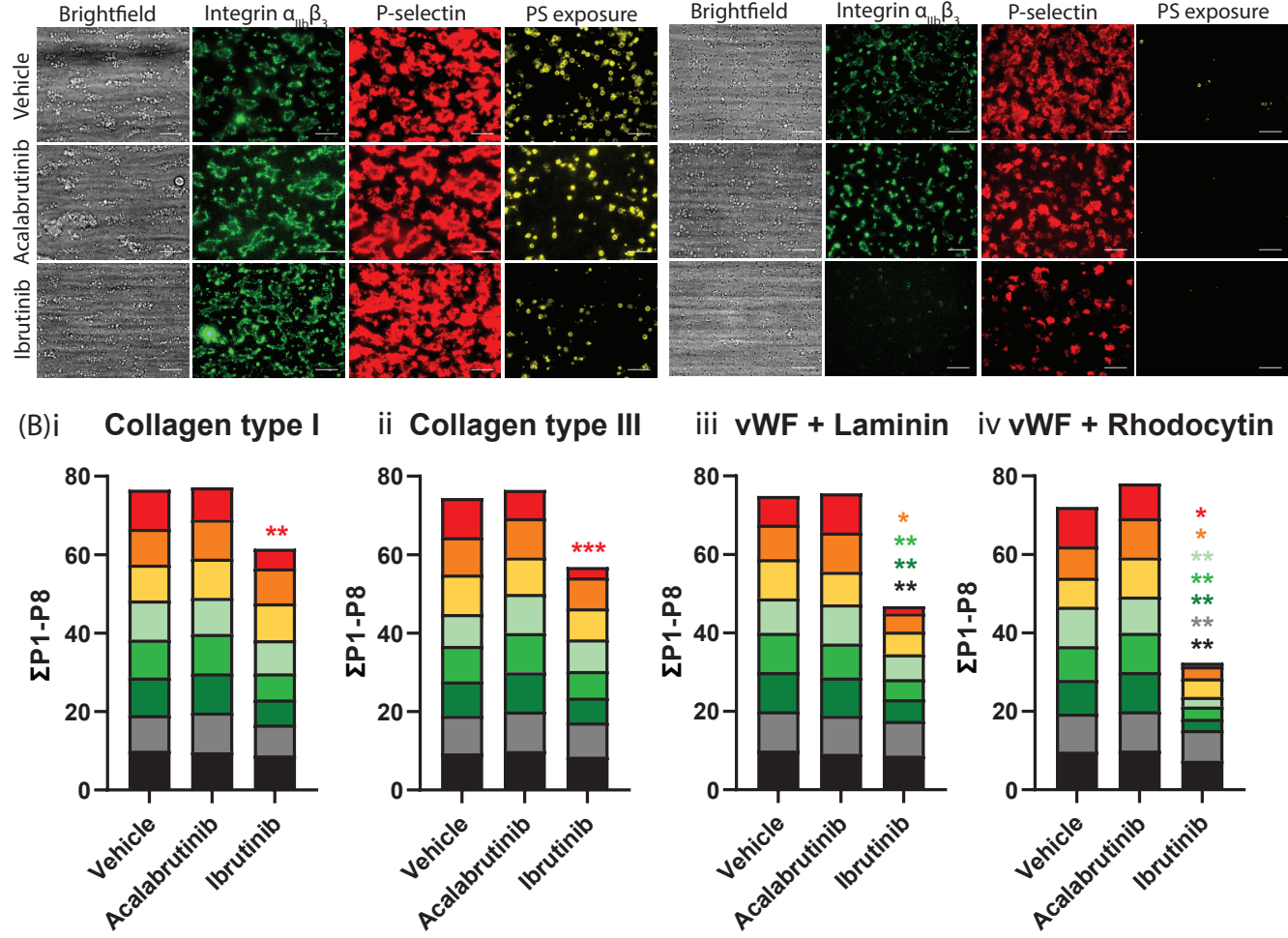

iii vWF + Laminin iv vWF + Rhodocytin
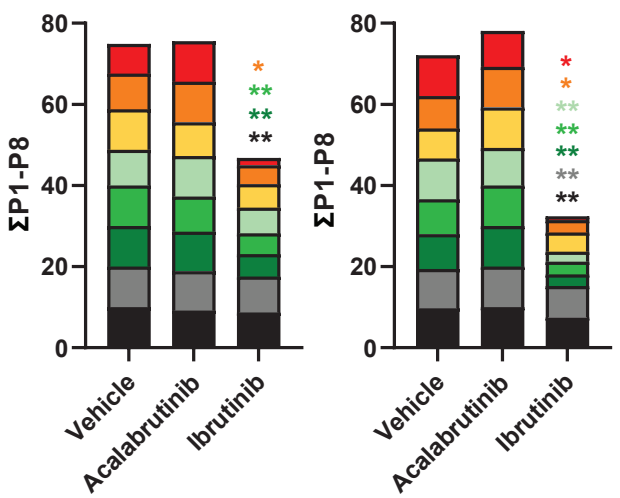

v vWF + Ristocetin vi vWF + Fibrinogen
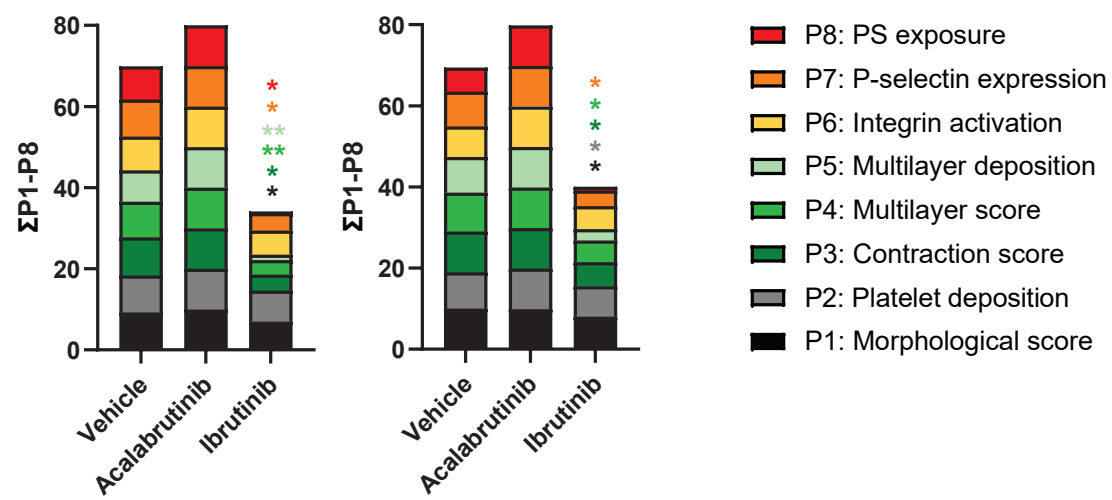

Figure 6.2. Ibrutinib, but not acalabrutinib, affected thrombus formation in whole blood perfused over six different surfaces. Pre-incubated blood samples from healthy donors with vehicle ( $<0.1 \%$ DMSO), acalabrutinib $(5 \mu \mathrm{M})$ or ibrutinib $(5 \mu \mathrm{M})$ were recalcified in presence of PPACK. Blood was perfused for 3.5 minutes at a wall shear rate of $1,000 \mathrm{~s}^{-1}$ over six different surfaces. After perfusion, thrombi were stained with AF568-annexin A5, FITC- $\alpha$-fibrinogen and AF647- $\alpha$-CD62P to detect PS-exposure, integrin $\alpha_{\| 1} \beta_{3}$ activation and P-selectin expression. (A) Representative images of thrombi formed on collagen type I and VWF co-coated with rhodocytin. (B) Histograms show the cumulative representation of scaled values from 0-10 for each parameter over different surfaces consisting of (i) collagen type I, (ii) collagen type III, (iii) VWF co-coated with laminin, (iv) VWF co-coated with rhodocytin, (v) VWF co-coated with ristocetin and (vi) VWF co-coated with fibrinogen in presence of acalabrutinib or ibrutinib. Colours reflect the adhesion parameters (P1-2; black, grey), aggregation parameters (P3-5; shades of green), and activation parameters (P6-8; yellow, orange and red). ${ }^{*} \mathrm{p}<0.05, * *$ $p<0.01, * * * p<0.001$ vs vehicle. 


\section{Effects of ibrutinib and acalabrutinib on wild-type and Btk-deficient mouse platelets}

To further examine the effects of ibrutinib and acalabrutinib on platelets, thrombus formation and platelet activation were determined in blood from WT and Btk-KO mice. Blood samples were incubated with ibrutinib or acalabrutinib before perfusion over collagen type I. In control conditions (vehicle), thrombus formation was reduced in blood from Btk-KO mice with regard to platelet deposition, multilayer formation (P1-2, P5) and PS-exposure (P8, Fig 6.3AB, Suppl Fig 6.3A). In WT blood, presence of acalabrutinib gave a similar inhibitory pattern of thrombus formation (Fig 6.3A, C). Comparable to human blood, incubation of WT mouse blood with ibrutinib resulted in limited effects on thrombus formation, only a decrease in PS-exposure was observed (Fig 6.3A, C, Suppl Fig 6.3A). When Btk inhibitors were pre-incubated in blood from Btk-KO mice, no additional inhibition on thrombus formation was observed (Figure 6.3A, C, suppl Fig 6.3A). The only observed effect, was a reduction in P-selectin expression in the presence of ibrutinib, as compared to vehicle, in Btk-KO mice (Figure 6.3A, C, Suppl Fig 6.3).

GPVI-induced platelet activation by CRP-XL was strongly reduced in Btk-KO platelets as compared to WT with regard to $\alpha_{\| 1} \beta_{3}$ activation, $\alpha$-granule release and PS-exposure, as determined by flow cytometry (Fig 6.3D-E). This was in agreement with the results obtained from whole blood perfusion experiments. Interestingly, $\alpha_{\| 1} \beta_{3}$ activation and P-selectin expression were also moderately reduced in Btk-KO mice upon PAR4 stimulation, while activation with ADP was not affected (Suppl Fig 6.3B-C). When blood was preincubated with Btk inhibitors, we observed significant reduction of all platelet activation markers in WT platelets upon GPVI stimulation (Fig 6.3D-E). In platelets from Btk-KO mice, both inhibitors did not further reduce $\alpha_{\| 1 \mathrm{~b}} \beta_{3}$ activation and P-selectin expression regardless of the agonist. We only observed significant reduction of PSexposure in Btk-KO platelets in the presence of ibrutinib, as compared to vehicle (Fig 6.3E).

Overall, preincubation of whole blood from Btk-KO mice with acalabrutinib and ibrutinib did not result in major additional effects on thrombus formation on collagen under flow, as well as on platelet activation.

\section{Btk inhibitors reduce GPVI-induced phosphorylation of multiple platelet tyrosine kinases}

To evaluate off-target effects of the Btk inhibitors, we performed a PamGene kinase assay to visualize which tyrosine kinases were regulated upon platelet stimulation via GPVI, as well as those tyrosine kinases that were subsequently influenced upon pre-incubation of the platelets with $1 \mu \mathrm{M}$ ibrutinib or acalabrutinib. We observed that in isolated platelets, in total 73 tyrosine kinases were significantly phosphorylated by stimulation with $\mathrm{CRP}^{65}$, of which were significantly inhibited by ibrutinib and acalabrutinib preincubation (Fig 6.4). Median kinase statistics showed that ibrutinib inhibited tyrosine phosphorylation of these proteins on average 4.4-fold, while acalabrutinib was less strong and reduced this response 1.8-fold, i.e. 2.5-fold weaker as compared to ibrutinib. 


\section{(A)}

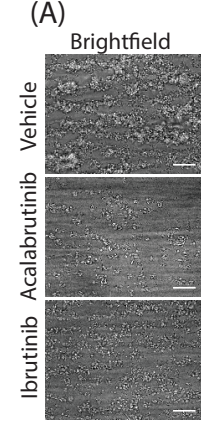

WT mice

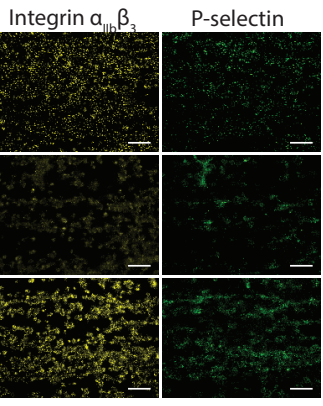

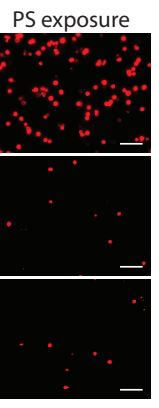

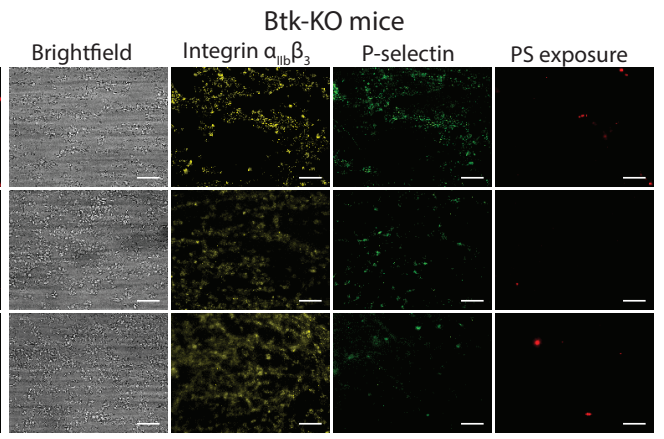

(B) Collagen type I

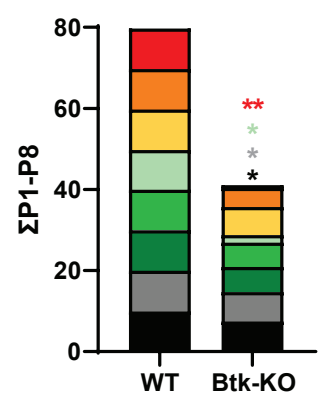

(C)

\section{Collagen type I}

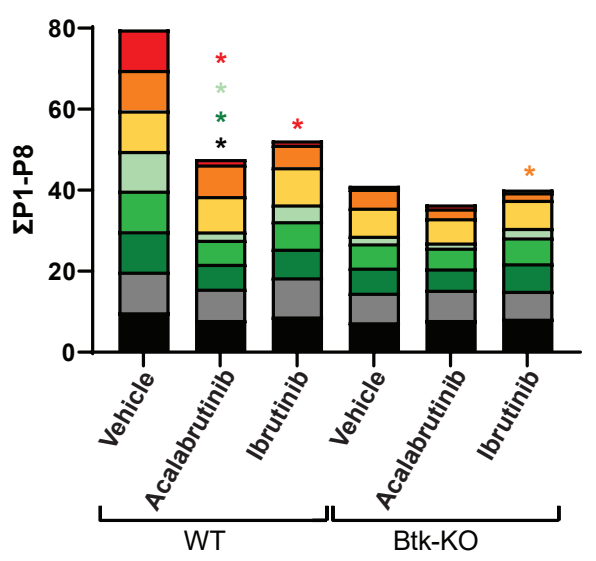

P8: PS exposure

口 P7: P-selectin expression

$\square$ P6: Integrin activation

$\square$ P5: Multilayer deposition

口 P4: Multilayer score

口 P3: Contraction score

口 P2: Platelet deposition

- P1: Morphological score

(D)

CRP-XL

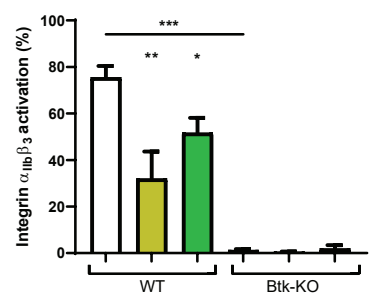

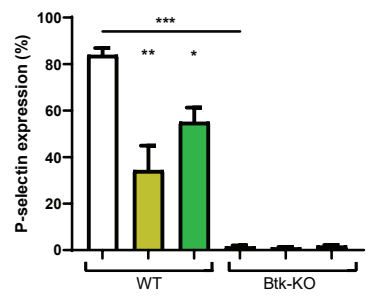

(E) CRP-XL

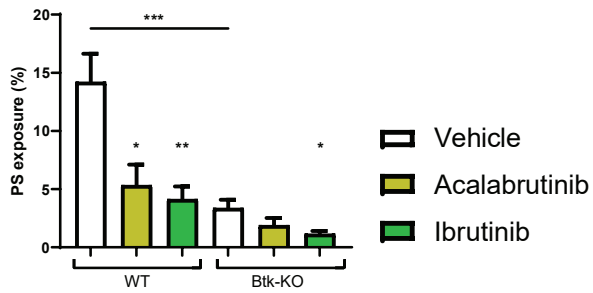

Figure 6.3. Acalabrutinib and ibrutinib reduced thrombus formation and PS-exposure in whole blood under flow, as well as activation of isolated platelets from WT but not Btk-KO mice. Pooled citrated whole blood samples from two WT or Btk-KO mice were pre-incubated with vehicle $(<0.1 \% \mathrm{DMSO})$, acalabrutinib $(5 \mu \mathrm{M})$ or ibrutinib $(5 \mu \mathrm{M})$ in the presence of PPACK and fragmin for 10 minutes at $37^{\circ} \mathrm{C}$. After incubation, blood samples were recalcified and perfused for 3.5 minutes at a wall shear rate of $1,000 \mathrm{~s}^{-1}$ over a collagen type I surface. Thrombi were post-stained with FITC-labelled anti-CD62P $\mathrm{mAb}$, PE-labelled JonA mAb and AF647-labelled Annexin-A5 to detect P-selectin expression, integrin activation and PSexposure respectively. (A) Representative brightfield and fluorescence images from thrombus formation in blood from WT and Btk-KO mice for each inhibitor. (B) Histograms show the cumulative representation of scaled values from 0-10 for each parameter on a collagen type I surface of thrombus formation in WT and Btk-KO mice blood samples. Colours reflect the adhesion parameters (P1-2; black, grey), aggregation parameters (P3-5; shades of green), and activation parameters (P68; yellow, orange and red). (C) Histograms of cumulative scaled values of multiple parameters in WT or Btk-KO mice with addition of vehicle ( $<0.1 \%$ DMSO), acalabrutinib or ibrutinib. (D) Platelet activation in whole blood induced by CRP-XL (1 $\mu \mathrm{g} / \mathrm{mL}$ ). Histograms show the percentages of platelets binding PE-labelled JON/A monoclonal antibody against activated $\alpha_{11 b} \beta_{3}$ integrin or BB700-labelled CD62P mAb in WT and BtK-KO mice. (E) PS-exposure of platelets induced by CRP-XL (1 $\mu \mathrm{g} /$ $\mathrm{mL}$ ). Histogram shows percentage of platelets binding FITC-labelled lactadherin. Data are shown as means \pm SEM $(n=6-8)$. ${ }^{*} p<0.05 .{ }^{* *} p<0.01,{ }^{* * *} p<0.001$ vs vehicle or otherwise indicated. 


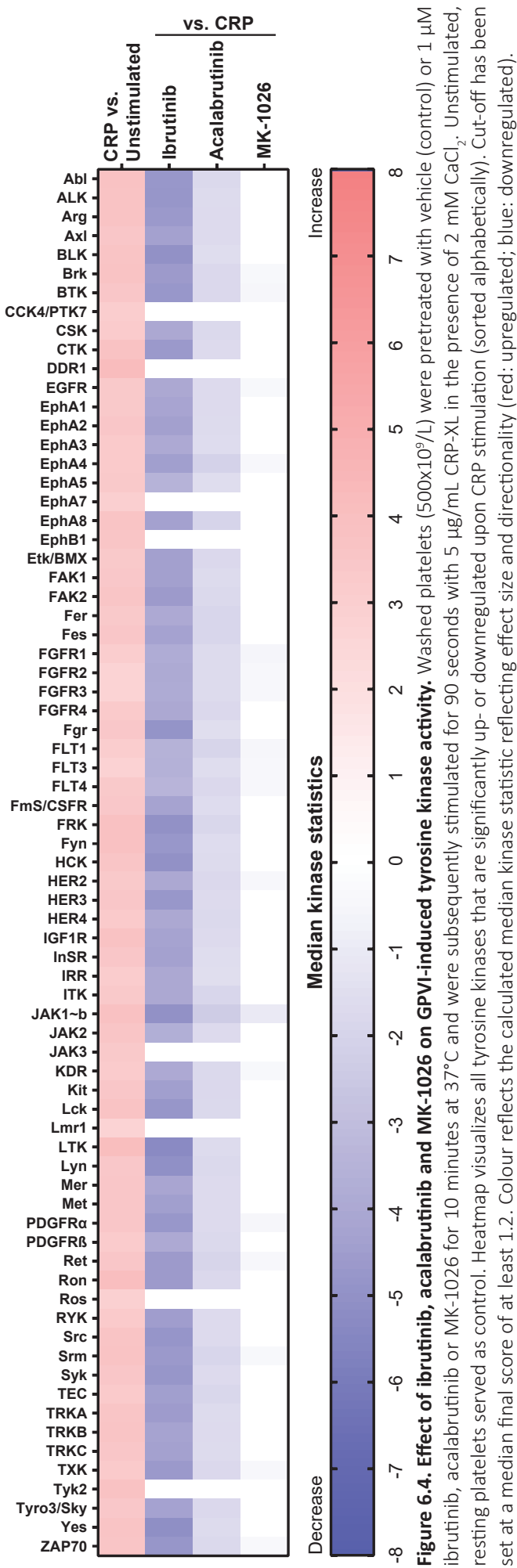




\section{Chapter 6}

In comparison to these irreversible inhibitors, we investigated if the reversible Btk inhibitor MK-102614 showed a more favourable anti-platelet effect. First, we evaluated which platelet tyrosine kinases were affected by this compound upon GPVI stimulation. Only 18 tyrosine kinases were significantly inhibited by MK-1026 (Fig 6.4). Median kinase statistics showed that MK-1026 inhibited tyrosine phosphorylation of these proteins on average 0.52 -fold, which was 8.3-fold lower as compared to ibrutinib. As expected, all three inhibitors significantly inhibited the phosphorylation of Btk, and ibrutinib and acalabrutinib also inhibited Tec. Furthermore, ibrutinib and acalabrutinib also inhibited the phosphorylation of Src family kinase (SFKs) and other downstream proteins as previously described ${ }^{11,12}$.

These results show that ibrutinib and acalabrutinib showed the same off-target effects on GPVI-induced tyrosine kinase phosphorylation in platelets, although the effects of acalabrutinib were less strong. The reversible inhibitor MK-1026 appeared to have less off-target effects in platelets upon GPVI stimulation, in combination with a less strong inhibition profile as compared to the irreversible inhibitors.

\section{The reversible Btk inhibitor MK-1026 shows only limited anti-platelet effects in human and mouse platelets in vitro}

As we demonstrated that irreversible inhibition of Btk can impair platelet function, we also investigated the effects of the reversible Btk inhibitor MK-1026 on platelet function. First, we performed collagen- and rhodocytin-induced dose-response aggregation experiments in PRP and washed platelets. Similar to the irreversible Btk inhibitors, MK-1026 inhibited platelet aggregation (with more prominent effects in washed platelets compared to PRP) at multiple concentrations induced by both agonists (Fig 6.5A). GPVI-induced integrin $\alpha_{\| 1} \beta_{3}$ activation, P-selectin expression, and PS-exposure were also dose-dependently reduced by MK-1026 (Fig 6.5B). The calculated $I_{50}$ values for MK-1026 in the presence of plasma were similar as for acalabrutinib (Suppl Table 6.1). Based on these results, in combination with reported in vivo plasma concentrations ${ }^{26}$, we selected $5 \mu \mathrm{M}$ for further experiments. Aggregation induced by ADP and U46619 were slightly but significantly reduced at this concentration of MK-1026, while responses to thrombin and ristocetin were unaffected (Suppl Fig 6.4A-B). Integrin $\alpha_{\| 1} \beta_{3}$ activation and P-selectin expression were not inhibited by MK-1026 in platelets stimulated with ADP or thrombin (Suppl Fig 6.4C). Also, MK-1026 did not alter platelet adhesion and thrombus formation on six different surfaces, as seen with acalabrutinib (Fig 6.5D, Suppl Fig 6.5).

We also investigated the effects of MK-1026 using blood from Btk-KO mice. Similarly as for acalabrutinib and ibrutinib, we did not observe additional inhibition of whole blood thrombus formation on collagen with MK-1026 in blood samples from WT or Btk-KO mice (Fig 6.6A-B, Suppl Fig 6.6A). Also, no inhibitory effect of MK-1026 was found upon platelet activation or PS-exposure from WT or Btk-KO mice with CRP-XL, ADP or PAR4 agonist (Fig 6.6C-D, Suppl Fig 6.6B-C). 

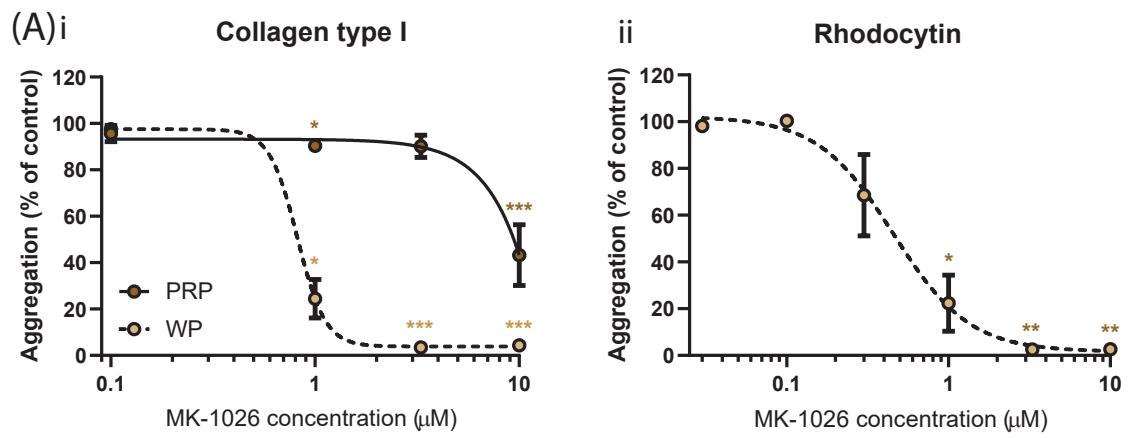

(B) $\mathrm{i}$

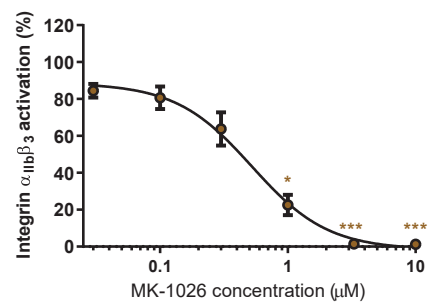

(D)i Collagen type I

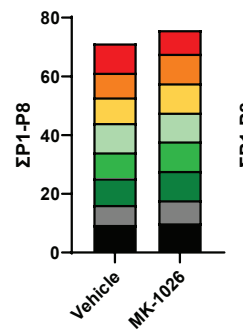

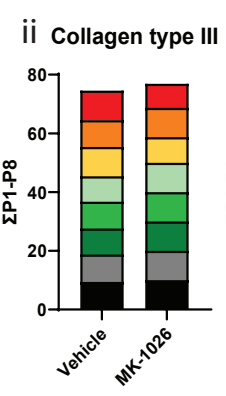

ii
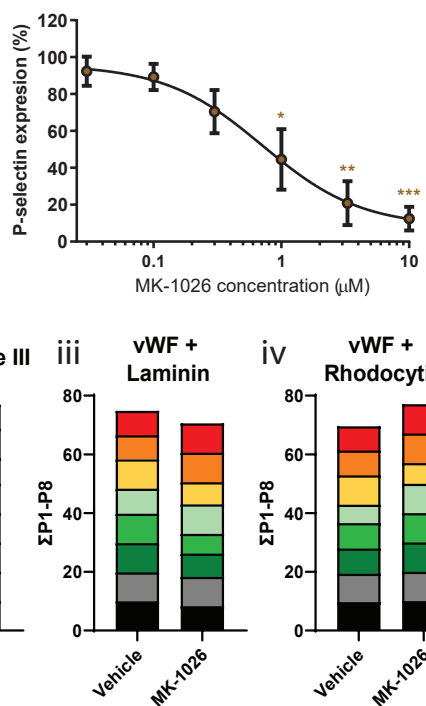
iv vWF +
(C) CRP-XL + Thrombin

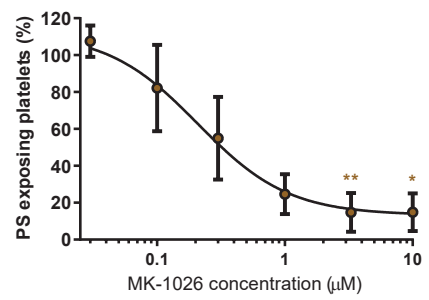

P1: Morphological score P2: Platelet deposition

P3: Contraction score
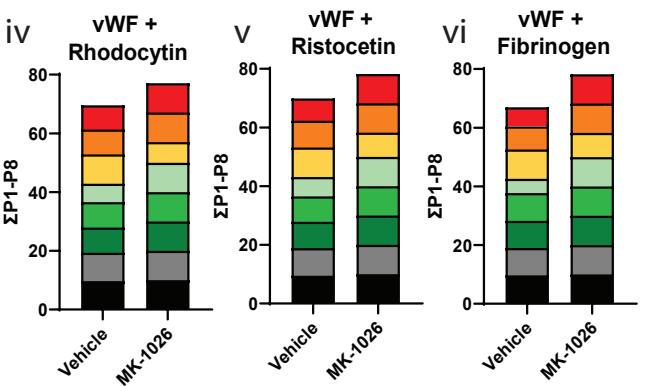

Figure 6.5. Effects of MK-1026 on aggregation and activation of isolated platelets in response to several agonists as well as whole blood thrombus formation under flow. (A) PRP or washed platelets $\left(250 \times 10^{9} / \mathrm{L}\right)$ from healthy volunteers were incubated with different concentrations of MK-1026 for 10 minutes at $37^{\circ} \mathrm{C}$. Shown are dose-response curves of aggregation induced by (i) collagen type I $(1 \mu \mathrm{g} / \mathrm{mL})$, or (ii) rhodocytin $(1 \mu \mathrm{g} / \mathrm{mL})$ in the presence of MK-1026 normalised to control expression. (B) Washed platelets $\left(100 \times 10^{9} / \mathrm{L}\right)$ were pre-incubated with different concentrations of MK-1026 and stimulated with collagen-related peptide (CRP-XL, $5 \mu \mathrm{g} / \mathrm{mL}$ ) for 15 minutes and simultaneously stained for activated integrin $\alpha_{\| 1} \beta_{3}$ and $\alpha$-granule secretion (P-selectin). Histograms show the percentages of platelets binding FITC-labelled PAC-1 monoclonal antibody or AF647-labelled anti-human CD62 mAb normalized to control expression. (C) Platelets pretreated with different concentrations of MK-1026 were stimulated using both CRP-XL $(5 \mu \mathrm{g} / \mathrm{mL})$ and thrombin ( $4 \mathrm{nM})$ for 60 minutes at $37^{\circ} \mathrm{C}$ and PS-exposure was determined by flow cytometry. Histogram shows percentage of platelets binding FITC-labelled annexin A5 normalized to control expression. Data are shown as means \pm SEM ( $n=6-7)$. (D) Pre-incubated blood samples from healthy donors with vehicle (<0.1\% DMSO) or MK-1026 (5 $\mu \mathrm{M})$ were recalcified in the presence of PPACK. Blood was perfused for 3.5 minutes at wall shear rate of 1,000 s-1 over six different coated surfaces. Thrombi were post-stained with AF568-annexin A5, FITC- $\alpha$-fibrinogen and AF647- $\alpha$-CD62P to detect PS-exposure, integrin activation and P-selectin expression. Cumulative histograms represent the scaled values (0-10) for each of the parameters. Colours reflect the adhesion parameters (P1-2; black, grey), aggregation parameters (P3-5; shades of green), and activation parameters (P6-8; yellow, orange and red). ${ }^{*} p<0.05,{ }^{* *} p<0.01, * * * p<0.001$ vs vehicle. 
These data show that the reversible Btk inhibitor MK-1026 inhibited GPVI- and CLEC-2mediated aggregation of washed platelets, but did not impair platelet adhesion or activation in whole blood perfused over collagen.

(A)

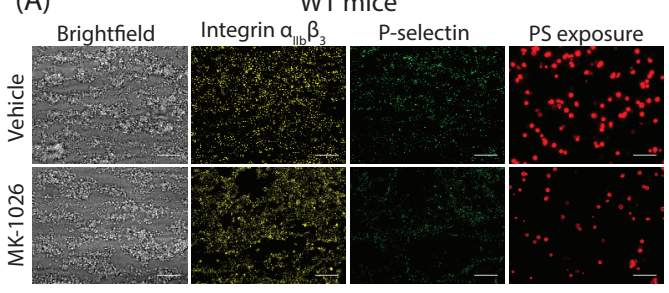

WT mice

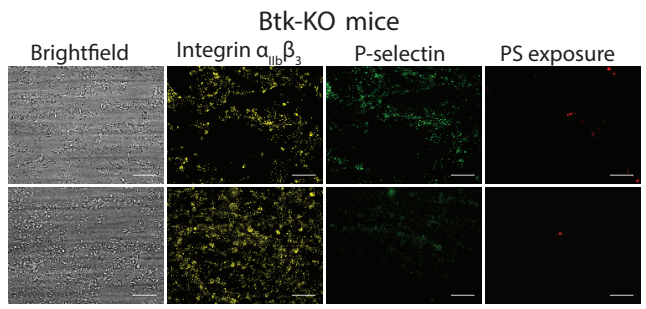

(B)

\section{Collagen type I}
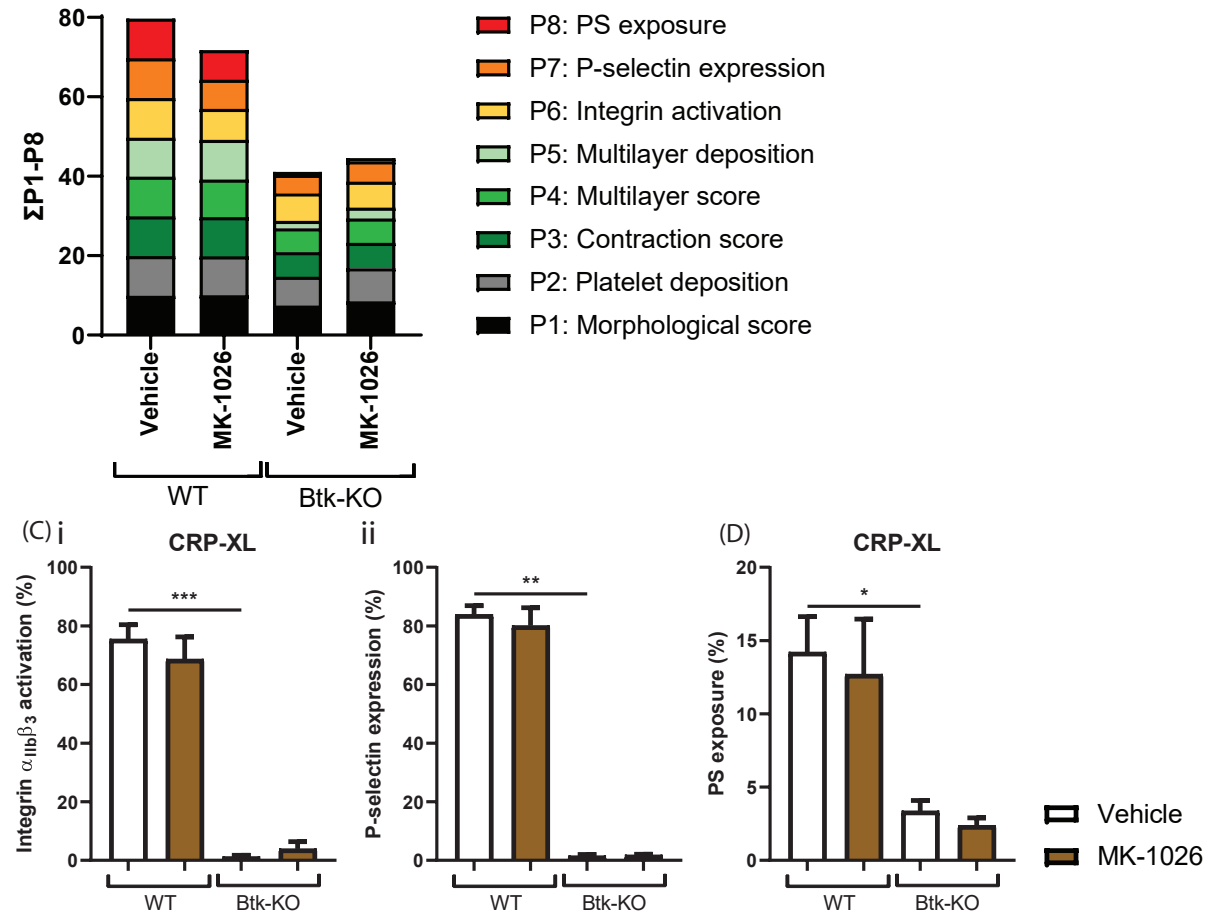

Figure 6.6. Thrombus formation, platelet activation and PS-exposure in blood from WT and Btk-KO mice was unaffected by MK-1026. Pooled citrated whole blood samples from two WT or Btk-KO mice were pre-incubated with vehicle $\left(<0.1 \%\right.$ DMSO) or MK-1026 $(5 \mu \mathrm{M})$ in the presence of PPACK and fragmin for 10 minutes at $37^{\circ} \mathrm{C}$. After incubation, blood samples were recalcified and perfused for 3.5 minutes at a wall shear rate of $1,000 \mathrm{~s}^{-1}$ over a collagen type I surface. Thrombi were post-stained with FITC-labelled anti-CD62P mAb, PE-labelled JON/A mAb and AF647-labelled Annexin-A5. (A) Representative brightfield and fluorescence images from thrombus formation in blood from WT and Btk-KO mice. (B) Histograms of cumulative scaled values of multiple parameters in WT or Btk-KO mice with addition of vehicle $(<0.1 \%$ DMSO) or MK-1026. Colours reflect the adhesion parameters (P1-2; black, grey), aggregation parameters (P3-5; shades of green), and activation parameters (P6-8; yellow, orange and red). (C) Platelet activation in whole blood induced by CRP$\mathrm{XL}(1 \mathrm{\mu g} / \mathrm{mL})$. Histograms show the percentages of platelets binding PE-labelled JON/A monoclonal antibody or BB700labelled CD62P mAb in WT and Btk-KO mice. (D) PS-exposure of platelets induced by CRP-XL (1 $\mu \mathrm{g} / \mathrm{mL})$. Histogram shows percentage of platelets binding FITC-labelled lactadherin. Data are shown as means \pm SEM $(n=6-8) .{ }^{*} p<0.05,{ }^{* *} p<0.01$, $* * * p<0.001$. 


\section{Ibrutinib inhibits thrombus formation under flow on multiple surfaces and GPVI-induced platelet activation responses in patients}

Next, we assessed which platelet activation pathways were altered in patients using ibrutinib. Sixteen patients were included, of which 7 did not receive ibrutinib and 9 were treated with 420-560 mg ibrutinib once a day (Table 6.1). Baseline characteristics were largely similar between groups. Importantly, none of the patients were thrombocytopenic, except one patient with a platelet count of $50 \times 10^{9} / \mathrm{L}$ (Table 6.1). Of the ibrutinib-treated patients, $66 \%$ (6/9) reported bleeding symptoms with a median ISTH-BAT score of 2 (range 1-7, Table 6.1). Of patients without ibrutinib treatment, none reported bleeding symptoms.

Whole blood from patients was perfused over collagen type I or vWF plus rhodocytin or laminin. No differences in thrombus formation were observed between patients without ibrutinib and healthy controls (Suppl Fig 6.7). On collagen, treatment with ibrutinib reduced thrombus contraction and height (multilayer) compared to non-treated patients. Furthermore, $\alpha_{\| 1} \beta_{3}$ activation, P-selectin expression and PS-exposure were significantly reduced (Fig 6.7A, Suppl Fig 6.7A). For thrombi formed on vWF plus rhodocytin or laminin, $\alpha_{\| 1} \beta_{3}$ activation and $\alpha$-granule secretion were reduced upon ibrutinib treatment (Fig 6.7A, Suppl Fig 6.7B-C).

Aggregation of isolated platelets from ibrutinib-treated patients showed on average no significant inhibition upon stimulation with any agonist (Fig 6.7B). However, platelets from 5 ibrutinib-treated patients were highly nonresponsive to collagen stimulation, while platelets from 2 patients responded normally to collagen. Also, with rhodocytin, platelets from 4 patients receiving ibrutinib did not aggregate, while platelets from 4 patients showed normal aggregation. When platelet activation was examined by flow cytometry, $\alpha_{11 b} \beta_{3}$ activation and P-selectin expression were inhibited in CRP-XL-stimulated platelets from all ibrutinib-treated patients (Fig 6.7C). Upon ADP stimulation, platelets from patients on treatment showed a slight increase in $\alpha_{\| 1} \beta_{3}$ activation, while no effects on secretion were observed (Fig 6.7C). PS-exposure by thrombin plus CRP-XL, was unchanged for all patients' platelets (Fig 6.7D).

As $66 \%$ of our patients reported bleeding symptoms, we compared patients with significant bleeding (>2 ISTH-BAT score) with patients with no/mild bleeding (0-1 ISTH-BAT score). Generally, no significant changes were observed in platelet aggregation and thrombus formation in patients with or without bleeding (Suppl Fig 6.8).

In summary, platelets from patients on ibrutinib treatment displayed inhibition of activation by GPVI as well as reduced adhesion and thrombus formation under flow over collagen and surfaces co-coated with vWF. Furthermore, variable responses were seen with CLEC-2 stimulation. 


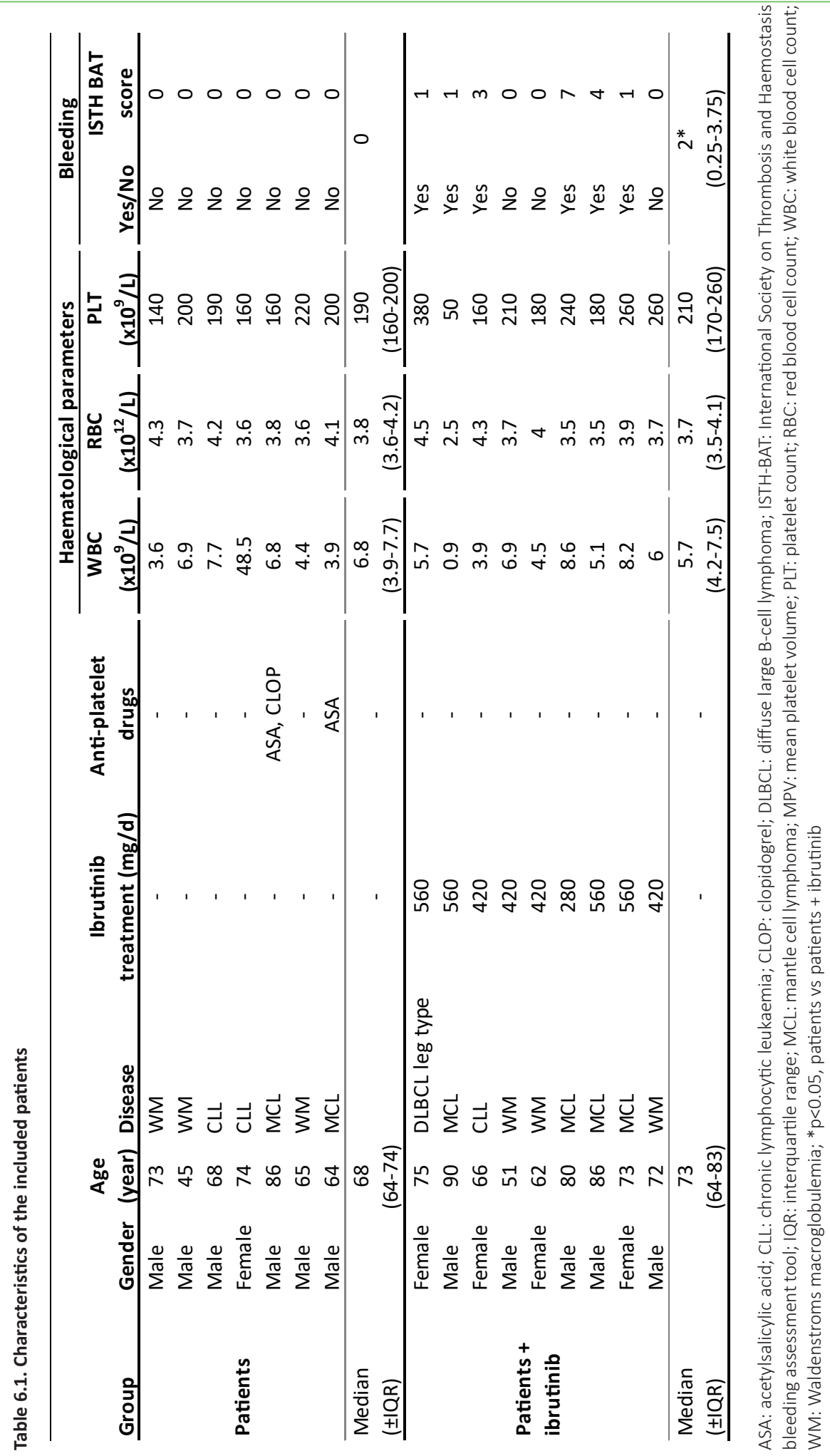




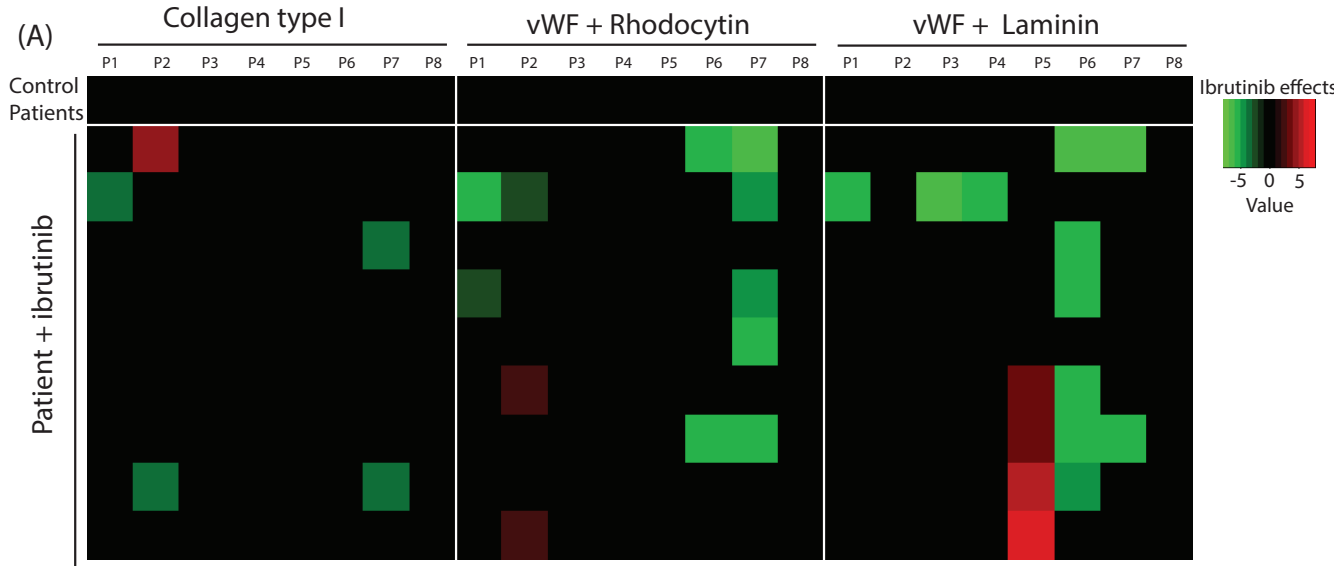

P1: Morphological score P3: Contraction score P5: Multilayer deposition P7: P-selectin expression P2: Platelet deposition P4: Multilayer score P6: Integrin activation P8: PS exposure

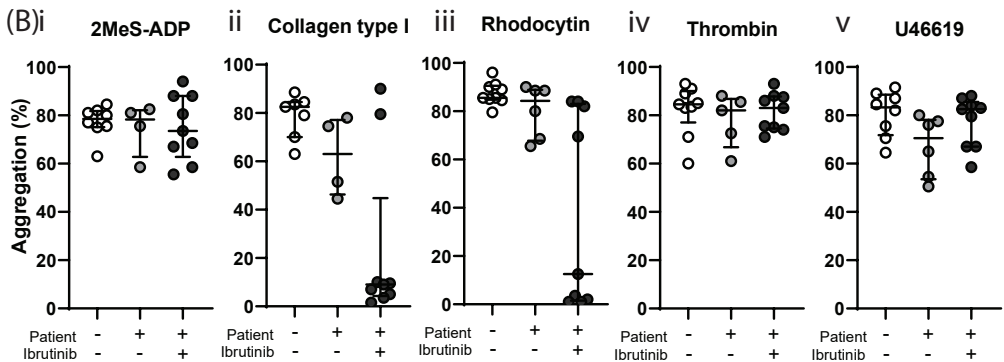

(C) CRP-XL

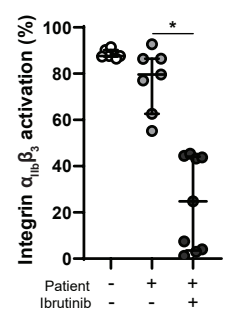

2MeS-ADP

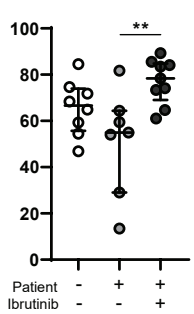

(D)

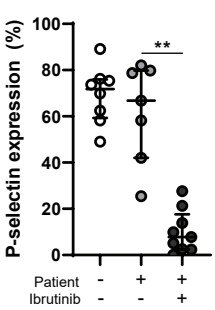

2MeS-ADP

(E) CRP-XL + Thrombin

Figure 6.7. Ibrutinib inhibits whole blood thrombus formation under flow on multiple surfaces and GPVI-induced platelet aggregation and activation responses in patients. Citrated whole blood was collected from healthy volunteers and 16 patients with(out) ibrutinib treatment. (A) Whole blood from healthy controls and patients was perfused for 3.5 minutes at a wall shear rate of $1,000 \mathrm{~s}^{-1}$ over coated microspots containing collagen type I, VWF co-coated with rhodocytin or VWF co-coated with laminin. Subtraction heatmap representing the effect of ibrutinib treatment in individual patients. Average values obtained with blood of patients without ibrutinib treatment was set at 0 for reference. Effects were filtered for changes greater than the 2x SD range of the different platelet parameters. (B) Light transmission aggregometry was induced in isolated platelets $\left(250 \times 10^{9} / \mathrm{L}\right)$ by collagen $(1 \mu \mathrm{g} / \mathrm{mL})$, rhodocytin $(1 \mu \mathrm{g} / \mathrm{mL}), 2 \mathrm{MeS}-\mathrm{ADP}(1 \mu \mathrm{M}), \mathrm{U} 46619(1$ $\mu \mathrm{M})$ or thrombin $(1 \mathrm{nM})$. Scatter plots show the percentage of aggregation of healthy controls (white circles), patients (grey circles) or patients receiving ibrutinib (black circles). Each circle represents one individual. (C, D) Washed platelets $\left(100 \times 10^{9} / \mathrm{L}\right)$ were stimulated with collagen-related peptide (CRP-XL, $\left.5 \mu \mathrm{g} / \mathrm{mL}\right)$ or $2 \mathrm{MeS}-\mathrm{ADP}(1 \mu \mathrm{M})$ for 15 minutes and analysed by flow cytometry. Shown are percentages of platelets binding FITC-labelled PAC1 mAb against integrin $\alpha_{\| b} \beta_{3}$ (C) and AF647-labelled CD62P mAb for $\alpha$-granule secretion (D), respectively. (E) PS-exposure was stimulated using both CRP-XL $(5 \mu \mathrm{g} / \mathrm{mL})$ and thrombin $(4 \mathrm{nM})$ for 60 minutes at $37^{\circ} \mathrm{C}$. Scatter plots show the percentage of platelets binding FITClabelled Annexin A5. Data are represented as median \pm interquartile range $(n=4-9), * p<0.05, * * p<0.01$. 


\section{Discussion}

Due to their promising effects on progression-free survival, Btk inhibitors are increasingly prescribed in haematological malignancies ${ }^{4,5}$. Btk inhibitors require lifelong treatment, and the high percentage of discontinuation due to side effects (including bleeding tendency) stresses the importance of understanding and managing these $e^{6-8}$. We found that ibrutinib and acalabrutinib inhibit both GPVI- and CLEC-2-mediated platelet aggregation in a dose-dependent manner. Also, all inhibitors dose-dependently impaired GPVI-induced $\alpha_{\| 1 b} \beta_{3}$ activation and $\alpha$-granule secretion. Ibrutinib reduced thrombus formation on surfaces co-coated with $v W F$, but not on collagen. Moreover, we are the first to study the effects on platelet function of MK-1026, a reversible Btk inhibitor.

Other studies investigating effects of Btk inhibitors on platelet function have described different findings. Several papers have shown inhibition of the GPVI pathway by ibrutinib or acalabrutinib, either directly via Btk or due to off-target effects on Src kinases or other downstream proteins $^{11,12,23,27-31}$. Others have implicated that GPIb-29, 32 or $\alpha_{116} \beta_{3}$-mediated signalling ${ }^{28,31}$ were affected by ibrutinib. In agreement, in the present study ibrutinib also inhibited thrombus formation on ristocetin and fibrinogen co-coated with vWF. Levade et al. have also shown that ibrutinib affected platelet adhesion to vWF only under flow, but did not assess acalabrutinib22. Furthermore, we found that both ibrutinib and acalabrutinib impaired GPVI-mediated aggregation, but not platelet adhesion on collagen under flow. Previous studies found conflicting results with regards to the effects of ibrutinib on collagen adhesion, with some who did find inhibition ${ }^{12,33}$ and others that could not ${ }^{34}$. A possible explanation for this is that platelet adhesion to collagen is also regulated by integrin $\alpha_{2} \beta_{1}$, which does not signal via Btk ${ }^{34}$. Our data now directly compared the differences between ibrutinib and acalabrutinib in whole blood thrombus formation under flow on six different surfaces, and shows for the first time that ibrutinib, but not acalabrutinib impairs platelet adhesion surfaces co-coated with vWF. This might play a role in the increased incidence of major bleeding seen in ibrutinib treatment compared to acalabrutinib.

Although GPVI is important for platelet adhesion and activation on collagen, recent studies have shown that GPVI inhibitors do not impair haemostasis ${ }^{16}$. GPVI-deficient patients exhibit only a mild bleeding diathesis ${ }^{17}$ and GPVI depletion in mice did not increase bleeding tendency ${ }^{19,20}$. Therefore, the bleeding tendency seen with Btk inhibitors cannot solely be mediated by GPVI. Depletion of both GPVI and CLEC-2 from mouse platelets did impair haemostasis ${ }^{21}$. In line with this, we found that Btk inhibitors inhibited both GPVI- and CLEC-2-mediated aggregation.

Only few studies have investigated Btk-KO mice or X-linked agammaglobulinemia (XLA) patients, a Btk deficiency in humans. Btk-KO mice showed impaired GPVI, CLEC-2 and GPIb signalling ${ }^{35-38}$. In line with this, we found decreased GPVI-induced platelet granule secretion and thrombus formation in Btk-KO mice. XLA patients demonstrated impaired GPVI- or CLEC-2-induced PLCY2 phosphorylation and platelet aggregation ${ }^{39}, 40$. As compared to XLA patients, ibrutinib has 
similar effects on GPVI, CLEC-2 and GPIb signalling. However, XLA patients are not associated with an increased bleeding risk ${ }^{12}$. This suggests that Btk inhibitors affect other targets, most notably Tec, which can substitute for Btk ${ }^{35}$ and is also inhibited by ibrutinib and acalabrutinib ${ }^{12}$. Also, offtarget effects on Src kinases have been implicated in the bleeding tendency ${ }^{11,12}$. We assessed offtarget effects on GPVI-induced tyrosine kinase phosphorylation in platelets by using the PamGene assay. These results showed that ibrutinib and acalabrutinib showed the same off-target effects, although the effects of acalabrutinib were less strong. Importantly both inhibitors had an off-target effect on Tec and Src kinases, questioning the previous conclusions that these were responsible for the increased severe bleeding seen with ibrutinib compared to acalabrutinib.

When comparing results found in vitro in human and mice, we found that in human samples, Btk inhibitors did not alter collagen-mediated adhesion under flow in agreement with Zheng et al. ${ }^{31}$, whereas in mice, Btk inhibition (either by KO mice or acalabrutinib) reduced this response. Btk-dependent signalling is completely absent in $\mathrm{KO}$ mice, and therefore, this may result in stronger effects as compared to pharmacological inhibition. Btk proteins display $99.4 \%$ similarity between human and mice $^{41}$, so a species-dependent interaction with an inhibitor cannot explain the observed differences. A possibility could be different bioavailability between mouse and man. Ibrutinib and acalabrutinib are highly plasma protein-bound in human blood, 97.3\% and $97.5 \%$, respectively ${ }^{42,43}$, while acalabrutinib was reported to have lower protein binding in mouse blood $(75.4 \%)^{43}$. This may contribute to the more pronounced effects of acalabrutinib in mouse blood as compared to human blood. We also observed different results between human and mouse platelets with MK-1026 (same concentration) in flow cytometric experiments. This may be explained by the use of murine whole blood in these experiments. Mouse blood contains $\sim$ three times more platelets as compared to human blood, and plasma proteins are also likely to bind MK-1026. Altogether, this may explain the absent effect of MK-1026 on platelet functions of wild-type mice.

As our data propose a direct role for Btk in impaired platelet function, we tested the reversible Btk inhibitor MK-1026. MK-1026 showed promising effects on ibrutinib resistant CLL cells in vitro and is currently being tested in clinical trials ${ }^{14}$. In the PamGene kinase assay MK1026 appeared to have less off-target effects in platelets upon GPVI stimulation, in combination with a less strong inhibition profile as compared to the irreversible inhibitors. MK-1026 reduced GPVI- and CLEC-2-mediated aggregation, as well as GPVI-induced platelet activation in washed platelets, largely similar to acalabrutinib. In human and mouse blood, MK-1026 did not impair collagen-induced thrombus formation under flow, in contrast to acalabrutinib which did affect this response in mouse blood. Hence, this reversible inhibitor had less inhibitory effects on platelets as compared to ibrutinib, in agreement with a recent study ${ }^{31}$. Hence, MK-1026 could be expected to show a slightly reduced or comparable bleeding tendency compared to acalabrutinib.

The present dose-response experiments, in line with previous studies ${ }^{11}{ }^{33}$, show that at 


\section{Chapter 6}

similar dose, ibrutinib is a more effective platelet inhibitor compared to other Btk inhibitors. This has been attributed to the inhibition of drug efflux pumps by ibrutinib ${ }^{33}$. We observed that higher concentrations of inhibitors are required to inhibit platelet aggregation in the presence of plasma, as compared to washed platelets. This in line with previous observations with other TKIs ${ }^{44,45}$. This is most likely caused by binding of ibrutinib and acalabrutinib to albumin ${ }^{46-48}$. It has been reported that prolongation of the incubation time lowered the $\mathrm{IC}_{50}$ values of ibrutinib and acalabrutinib for GPVI-mediated aggregation ${ }^{49}$. However, in that study a much lower collagen concentration was used, which may be more sensitive to longer incubation with low doses of inhibitors. Furthermore, that study did not include CLEC-2-dependent platelet responses ${ }^{49}$. Although inhibitor concentration could play a role in the extent of inhibition, we showed that ibrutinib and acalabrutinib influence additional platelet pathways as compared to MK-1026, suggesting that the extent of Btk inhibition might not be the reason for the increased bleeding tendency.

Previous studies assessing which platelet pathways are involved in bleeding tendency in patients using ibrutinib found conflicting results. Some showed a correlation with collageninduced aggregation ${ }^{12,22,50}$, and some with platelet adhesion to collagen ${ }^{12}$, whereas others could not find this ${ }^{22}$. Bleeding tendency was also associated with adhesion to VWF under flow ${ }^{22}$ and one study found a correlation with ristocetin-induced platelet aggregation ${ }^{32}$, which another study could not replicate ${ }^{28}$. Ibrutinib can also induce shedding of GPIb $\alpha$, GPIX and integrin $\alpha_{116} \beta_{3}$ in patients, but the correlation with bleeding remains unknown ${ }^{51}$.

A recent study assessed platelet parameters in patients with CLL and mantle cell lymphoma $(\mathrm{MCL})^{52}$, showing correlations between bleeding tendency, thrombocytopenia and decreased ADP-induced platelet aggregation. However, a drawback of this study was that a large patient proportion was thrombocytopenic, which can directly influence platelet aggregation, as platelet concentration in PRP was not reported to be adjusted. This impaired the establishment if ibrutinib or platelet count affected aggregation response. In our patients with (in general) normal platelet counts, we also observed significant differences in platelet responses to several stimuli of patients using ibrutinib. Furthermore, 66\% of the included patients with ibrutinib treatment reported bleeding symptoms. Although we could show that in patients, similar to healthy volunteers, ibrutinib inhibited GPVI signalling, with variable effects on CLEC-2, as well as reduced thrombus formation to surfaces co-coated with vWF, this could not differentiate for the bleeding tendency. Generally, the patients showed a large variation in measurement outcomes, which might be influenced by clinical factors. However, with patients on ibrutinib treatment, we have directly compared whole blood thrombus formation under flow on multiple surfaces, including vWF plus rhodocytin and laminin, which has not been reported with ibrutinib-treated patients thus far.

In conclusion, the present work demonstrated that ibrutinib, acalabrutinib and MK-1026 inhibited GPVI- and CLEC-2-mediated platelet aggregation, but only ibrutinib also inhibited GPVIinduced platelet activation and thrombus formation on surfaces co-coated with vWF. The novel 
reversible BTK inhibitor MK-1026 might therefore be promising for future treatment in patients at risk for bleeding.

\section{Acknowledgements}

TAMC was supported by the Heimburger award from CSL Behringer. JAE is financially supported by the Interdisziplinary Center of Clinical Research (IZKF) of the University of Münster (grant no. Ebl1/009/21). Additional support was received by ZonMW VIDI 91716421 to MFAK and JMEMC and by the Dutch Heart Foundation (2015T79 to JMEMC and 2020T020 to CCFMJB). CCFMJB was also supported by the START-Program of the Faculty of Medicine at the RWTH Aachen University (105/20). EPCVdV was supported by a grant from the Interdisciplinary Center for Clinical Research within the faculty of Medicine at the RWTH Aachen University, the Fritz Thyssen Stiftung (10.20.2.043MN), and NWO-ZonMw Veni (91619053). All authors declare no conflict of interest.

\section{Author contributions}

BMET, MFAK, VL, MStB, CCFMJB, SLM and EPCVdV acquired, analysed and interpreted data. AFdV provided Btk-deficient mice. JAE contributed essential tools. MRN and TAMC provided ethical approval and included patients. JMEMC and JWMH provided lab space with equipment and supervision. TAMC and MJEK designed and supervised the research, and interpreted data. BMET, TAMC and MJEK wrote and revised the paper. All the authors provided critical feedback, edited, and approved the final manuscript.

\section{References}

1. Byrd, JC, Furman, RR, Coutre, SE, Burger, JA, Blum, KA, Coleman, M et al. Three-year follow-up of treatment-naive and previously treated patients with CLL and SLL receiving single-agent ibrutinib. Blood. 2015; 125, 2497-2506.

2. Byrd, JC, Brown, JR, O'Brien, S, Barrientos, JC, Kay, NE, Reddy, NM et al. Ibrutinib versus ofatumumab in previously treated chronic lymphoid leukemia. N Engl J Med. 2014; 371, 213-223.

3. Wang, ML, Blum, KA, Martin, P, Goy, A, Auer, R, Kahl, BS et al. Long-term follow-up of MCL patients treated with single-agent ibrutinib: updated safety and efficacy results. Blood. 2015; 126, 739-745.

4. Castillo, JJ, Advani, RH, Branagan, AR, Buske, C, Dimopoulos, MA, D'Sa, S et al. Consensus treatment recommendations from the tenth International Workshop for Waldenström Macroglobulinaemia. Lancet Haematol. 2020; 7, e827-e837.

5. Hallek, M. Chronic lymphocytic leukemia: 2020 update on diagnosis, risk stratification and treatment. Am J Hematol. 2019; 94, 1266-1287.

6. Shatzel, JJ, Olson, SR, Tao, DL, McCarty, OJT, Danilov, AV \& DeLoughery, TG. Ibrutinib-associated bleeding: pathogenesis, management and risk reduction strategies. J Thromb Haemost. 2017; 15, 835-847.

7. Mato, AR, Roeker, LE, Allan, JN, Pagel, JM, Brander, DM, Hill, BT et al. Outcomes of front-line ibrutinib treated CLL patients excluded from landmark clinical trial. Am J Hematol. 2018; 93, 1394-1401.

8. Stephens, DM \& Byrd, JC. How I manage ibrutinib intolerance and complications in patients with chronic lymphocytic leukemia. Blood. 2019; 133, 1298-1307.

9. de Weerdt, I, Koopmans, SM, Kater, AP \& van Gelder, M. Incidence and management of toxicity 
associated with ibrutinib and idelalisib: a practical approach. Haematologica. 2017; 102, 1629-1639.

10. Brown, JR, Moslehi, J, Ewer, MS, O'Brien, SM, Ghia, P, Cymbalista, F et al. Incidence of and risk factors for major haemorrhage in patients treated with ibrutinib: An integrated analysis. Br J Haematol. 2019; 184, 558-569.

11. Nicolson, PLR, Hughes, CE, Watson, S, Nock, SH, Hardy, AT, Watson, CN et al. Inhibition of Btk by Btkspecific concentrations of ibrutinib and acalabrutinib delays but does not block platelet aggregation mediated by glycoprotein VI. Haematologica. 2018; 103, 2097-2108.

12. Bye, AP, Unsworth, AJ, Desborough, MJ, Hildyard, CAT, Appleby, N, Bruce, D et al. Severe platelet dysfunction in NHL patients receiving ibrutinib is absent in patients receiving acalabrutinib. Blood Adv. 2017; 1, 2610-2623.

13. Wen, T, Wang, J, Shi, Y, Qian, H \& Liu, P. Inhibitors targeting Bruton's tyrosine kinase in cancers: drug development advances. Leukemia. 2021; 35, 312-332.

14. Reiff, SD, Mantel, R, Smith, LL, Greene, JT, Muhowski, EM, Fabian, CA et al. The BTK Inhibitor ARQ 531 Targets Ibrutinib-Resistant CLL and Richter Transformation. Cancer Discov. 2018; 8, 1300-1315.

15. Byrd, JC, Harrington, B, O'Brien, S, Jones, JA, Schuh, A, Devereux, S et al. Acalabrutinib (ACP-196) in Relapsed Chronic Lymphocytic Leukemia. N Engl J Med. 2016; 374, 323-332.

16. Denorme, F \& Rondina, MT. Targeting Glycoprotein VI for Thromboembolic Disorders. Arterioscler Thromb Vasc Biol. 2019; 39, 839-840.

17. Matus, V, Valenzuela, G, Sáez, CG, Hidalgo, P, Lagos, M, Aranda, E et al. An adenine insertion in exon 6 of human GP6 generates a truncated protein associated with a bleeding disorder in four Chilean families. J Thromb Haemost. 2013; 11, 1751-1759.

18. Nagy, M, Perrella, G, Dalby, A, Becerra, MF, Garcia Quintanilla, L, Pike, JA et al. Flow studies on human GPVI-deficient blood under coagulating and noncoagulating conditions. Blood Adv. 2020; 4, 29532961.

19. Lockyer, S, Okuyama, K, Begum, S, Le, S, Sun, B, Watanabe, T et al. GPVI-deficient mice lack collagen responses and are protected against experimentally induced pulmonary thromboembolism. Thromb Res. 2006; 118, 371-380.

20. Nieswandt, B, Schulte, V, Bergmeier, W, Mokhtari-Nejad, R, Rackebrandt, K, Cazenave, JP et al. Longterm antithrombotic protection by in vivo depletion of platelet glycoprotein VI in mice. J Exp Med. 2001; 193, 459-469.

21. Bender, M, May, F, Lorenz, V, Thielmann, I, Hagedorn, I, Finney, BA et al. Combined in vivo depletion of glycoprotein $\mathrm{VI}$ and C-type lectin-like receptor 2 severely compromises hemostasis and abrogates arterial thrombosis in mice. Arterioscler Thromb Vasc Biol. 2013; 33, 926-934.

22. Levade, M, David, E, Garcia, C, Laurent, PA, Cadot, S, Michallet, AS et al. Ibrutinib treatment affects collagen and von Willebrand factor-dependent platelet functions. Blood. 2014; 124, 3991-3995.

23. Bye, AP, Unsworth, AJ, Vaiyapuri, S, Stainer, AR, Fry, MJ \& Gibbins, JM. Ibrutinib Inhibits Platelet Integrin alphallbbeta3 Outside-In Signaling and Thrombus Stability But Not Adhesion to Collagen. Arterioscler Thromb Vasc Biol. 2015; 35, 2326-2335.

24. de Witt, SM, Swieringa, F, Cavill, R, Lamers, MM, van Kruchten, R, Mastenbroek, T et al. Identification of platelet function defects by multi-parameter assessment of thrombus formation. Nat Commun. 2014; 5, 4257.

25. Advani, RH, Buggy, JJ, Sharman, JP, Smith, SM, Boyd, TE, Grant, B et al. Bruton tyrosine kinase inhibitor ibrutinib (PCl-32765) has significant activity in patients with relapsed/refractory B-cell malignancies. J Clin Oncol. 2013; 31, 88-94.

26. Elgamal, OA, Mehmood, A, Jeon, JY, Carmichael, B, Lehman, A, Orwick, SJ et al. Preclinical efficacy for a novel tyrosine kinase inhibitor, ArQule 531 against acute myeloid leukemia. J Hematol Oncol. 2020; $13,8$.

27. Ninomoto, J, Mokatrin, A, Kinoshita, T, Marimpietri, C, Barrett, TD, Chang, BY et al. Effects of ibrutinib on in vitro platelet aggregation in blood samples from healthy donors and donors with platelet dysfunction. Hematology. 2020; 25, 112-117.

28. Rigg, RA, Aslan, JE, Healy, LD, Wallisch, M, Thierheimer, ML, Loren, CP et al. Oral administration of Bruton's tyrosine kinase inhibitors impairs GPVI-mediated platelet function. Am J Physiol Cell Physiol. 2016; 310, C373-380. 
29. Kamel, S, Horton, L, Ysebaert, L, Levade, M, Burbury, K, Tan, S et al. Ibrutinib inhibits collagenmediated but not ADP-mediated platelet aggregation. Leukemia. 2015; 29, 783-787.

30. Alberelli, MA, Innocenti, I, Sica, S, Laurenti, L \& De Candia, E. PO-54 - Clinical and laboratory characterization of platelet dysfunction caused by ibrutinib treatment in patients with chronic lymphocytic leukemia. Thromb Res. 2016; 140.

31. Zheng, TJ, Lofurno, ER, Melrose, AR, Lakshmanan, HHS, Pang, J, Phillips, KG et al. Assessment of the effects of Syk and BTK inhibitors on GPVI-mediated platelet signaling and function. Am J Physiol Cell Physiol. 2021.

32. Kazianka, L, Drucker, C, Skrabs, C, Thomas, W, Melchardt, T, Struve, S et al. Ristocetin-induced platelet aggregation for monitoring of bleeding tendency in CLL treated with ibrutinib. Leukemia. 2017; 31, 1117-1122.

33. Series, J, Garcia, C, Levade, M, Viaud, J, Sie, P, Ysebaert, L et al. Differences and similarities in the effects of ibrutinib and acalabrutinib on platelet functions. Haematologica. 2019; 104, 2292-2299.

34. Busygina, K, Jamasbi, J, Seiler, T, Deckmyn, H, Weber, C, Brandl, R et al. Oral Bruton tyrosine kinase inhibitors selectively block atherosclerotic plaque-triggered thrombus formation in humans. Blood. 2018; 131, 2605-2616.

35. Atkinson, BT, Ellmeier, W \& Watson, SP. Tec regulates platelet activation by GPVI in the absence of Btk. Blood. 2003; 102, 3592-3599.

36. Pasquet, JM, Quek, L, Stevens, C, Bobe, R, Huber, M, Duronio, V et al. Phosphatidylinositol 3,4,5-trisphosphate regulates $\mathrm{Ca}(2+)$ entry via btk in platelets and megakaryocytes without increasing phospholipase C activity. EMBO J. 2000; 19, 2793-2802.

37. Liu, J, Fitzgerald, ME, Berndt, MC, Jackson, CW \& Gartner, TK. Bruton tyrosine kinase is essential for botrocetin/VWF-induced signaling and GPIb-dependent thrombus formation in vivo. Blood. 2006; 108, 2596-2603.

38. de Porto, A, Claushuis, TAM, van der Donk, LEH, de Beer, R, de Boer, OJ, Florquin, S et al. Platelet Btk is Required for Maintaining Lung Vascular Integrity during Murine Pneumococcal Pneumosepsis. Thromb Haemost. 2019; 119, 930-940.

39. Quek, LS, Bolen, J \& Watson, SP. A role for Bruton's tyrosine kinase (Btk) in platelet activation by collagen. Curr Biol. 1998; 8, 1137-1140.

40. Nicolson, PLR, Nock, SH, Hinds, J, Garcia-Quintanilla, L, Smith, CW, Campos, J et al. Low-dose Btk inhibitors selectively block platelet activation by CLEC-2. Haematologica. 2021; 106, 208-219.

41. Oeltjen, JC, Malley, TM, Muzny, DM, Miller, W, Gibbs, RA \& Belmont, JW. Large-scale comparative sequence analysis of the human and murine Bruton's tyrosine kinase loci reveals conserved regulatory domains. Genome Res. 1997; 7, 315-329.

42. Bose, P, Gandhi, VV \& Keating, MJ. Pharmacokinetic and pharmacodynamic evaluation of ibrutinib for the treatment of chronic lymphocytic leukemia: rationale for lower doses. Expert Opin Drug Metab Toxicol. 2016; 12, 1381-1392.

43. Podoll, T, Pearson, PG, Evarts, J, Ingallinera, T, Bibikova, E, Sun, Het al. Bioavailability, Biotransformation, and Excretion of the Covalent Bruton Tyrosine Kinase Inhibitor Acalabrutinib in Rats, Dogs, and Humans. Drug Metab Dispos. 2019; 47, 145-154.

44. Tullemans, BME, Nagy, M, Sabrkhany, S, Griffioen, AW, Oude Egbrink, MGA, Aarts, M et al. Tyrosine Kinase Inhibitor Pazopanib Inhibits Platelet Procoagulant Activity in Renal Cell Carcinoma Patients. Front Cardiovasc Med. 2018; 5, 142.

45. Tullemans, BME, Fernandez, DI, Veninga, A, Baaten, C, Peters, LJF, Aarts, MJB et al. Tyrosine Kinase Inhibitor Sunitinib Delays Platelet-Induced Coagulation: Additive Effects of Aspirin. Thromb Haemost. 2021.

46. Liu, X, Feng, D, Zheng, M, Cui, Y \& Zhong, D. Characterization of covalent binding of tyrosine kinase inhibitors to plasma proteins. Drug Metab Pharmacokinet. 2020; 35, 456-465.

47. Tang, B, Tang, P, He, J, Yang, H \& Li, H. Characterization of the binding of a novel antitumor drug ibrutinib with human serum albumin: Insights from spectroscopic, calorimetric and docking studies. J Photochem Photobiol B. 2018; 184, 18-26.

48. Abdelhameed, AS, Alanazi, AM, Bakheit, AH, Hassan, ES, Herqash, RN \& Almutairi, FM. Novel BTK inhibitor acalabrutinib (ACP-196) tightly binds to site I of the human serum albumin as observed by 
spectroscopic and computational studies. Int J Biol Macromol. 2019; 127, 536-543.

49. Denzinger, V, Busygina, K, Jamasbi, J, Pekrul, I, Spannagl, M, Weber, C et al. Optimizing Platelet GPVI Inhibition versus Haemostatic Impairment by the Btk Inhibitors Ibrutinib, Acalabrutinib, ONO/GS4059, BGB-3111 and Evobrutinib. Thromb Haemost. 2019; 119, 397-406.

50. Lipsky, AH, Farooqui, MZ, Tian, X, Martyr, S, Cullinane, AM, Nghiem, K et al. Incidence and risk factors of bleeding-related adverse events in patients with chronic lymphocytic leukemia treated with ibrutinib. Haematologica. 2015; 100, 1571-1578.

51. Dobie, G, Kuriri, FA, Omar, MMA, Alanazi, F, Gazwani, AM, Tang, CPS et al. Ibrutinib, but not zanubrutinib, induces platelet receptor shedding of GPIb-IX-V complex and integrin alphallbbeta3 in mice and humans. Blood Adv. 2019; 3, 4298-4311.

52. Dmitrieva, EA, Nikitin, EA, Ignatova, AA, Vorobyev, VI, Poletaev, AV, Seregina, EA et al. Platelet function and bleeding in chronic lymphocytic leukemia and mantle cell lymphoma patients on ibrutinib. J Thromb Haemost. 2020; 18, 2672-2684. 


\section{Supplemental Data}

\section{Materials \& methods}

\section{Materials}

Acalabrutinib was obtained from Selleckchem (Houston, TX, USA), MK-1026 (formerly ARQ-531) was a provided by ArQule, Inc., a wholly-owned subsidiary of Merck \& Co. (Kenilworth NJ, USA) and ibrutinib was purchased from Toronto Research Chemicals (North York, Canada). Bovine serum albumin (BSA), D(+)-glucose, unfractionated heparin and apyrase were purchased from Sigma-Aldrich (Saint Louis MO, USA). Low molecular weight heparin (Fragmin ${ }^{\circledR}$ ) was from Prizer (Capelle a/d IJssel, The Netherlands). Horm ${ }^{\circledR}$ collagen type I came from Takeda (Hoofddorp, The Netherlands), whereas the agonists collagen-related peptide crosslinked (CRP-XL) and von Willebrand factor III (vWF-III) were obtained from CambCol Laboratories (Cambridge, UK). Thrombin was from Enzyme Research Laboratories Inc (South Bend, IN, USA) and proteaseactivated receptor 4 activating peptide (PAR4-AP) was obtained from Bachem (Bubendorf, Switzerland). U46619 (thromboxane $A_{2}$ receptor agonist) came from Cayman Chemicals (Ann Arbor MI, USA). 2-Methylthio-adenosine-diphosphate (2MeS-ADP) and D-phenylalanyl-prolylarginyl chloromethyl ketone (PPACK) were obtained from Santa Cruz Biotechnology (Dallas TX, USA). Laminin was purchased from Octapharma (Lachen, Switzerland), whereas rhodocytin was purified from Calloselasma rhodostoma venom as described previously ${ }^{1}$. Fura-2-AM was obtained from Invitrogen (Carlsbad CA, USA). Fluorescein isothiocyanate (FITC)-labelled PAC1 monoclonal antibody (mAb) against activated human integrin $\alpha_{\| b} \beta_{3}$ were purchased from BD Bioscience (nr. 340507; Franklin Lakes NJ, USA). FITC-conjugated $\alpha$-fibrinogen mAb was purchased from DAKO (F0111; Santa Clara CA, USA). Alexa Fluor (AF)568 and AF647-conjugated annexin A5 were purchased from Molecular Probes, Life Technologies (New York NY, USA). AF647-labelled CD62-P mAb was obtained from Biolegend (London, UK). FITC-conjugated annexin A5 was from Pharmatarget (Maastricht, The Netherlands), whereas FITC-conjugated lactadherin came from Haematologic Technologies (Essex Junction VT, USA). FITC-labelled rat anti-mouse CD62P mAb and phycoerythrin (PE) labelled rat anti-mouse JON/A mAb were obtained from Emfret Analytics (Würzburg, Germany).

\section{Blood collection and platelet isolation from healthy volunteers and patients}

With approval from the medical ethics committee from the Maastricht University Medical Centre+ (MUMC+), blood was collected from healthy volunteers. Furthermore, blood was collected from patients diagnosed with chronic lymphocytic leukaemia (CLL), mantle cell lymphoma and Waldenstroms macroglobulinemia with or without ibrutinib treatment at the Catharina Hospital in Eindhoven (the Netherlands) with approval of the local medical ethical committee. This study was performed in accordance with the declaration of Helsinki and informed consent was obtained 


\section{Chapter 6}

from all participants. Blood was collected in 3.2\% trisodium citrate tubes by venipuncture, after discarding the first $3 \mathrm{~mL}$ of blood. Blood cell counts and haematological parameters were assessed of all blood samples using a Sysmex XP300 (Kobe, Japan). Patients characteristic and ISTH-BAT score2 were acquired for each patient.

Platelet-rich plasma (PRP) or platelets were isolated from whole blood as described previously3, ${ }^{3}$. Washed platelets were resuspended in Hepes buffer, pH 7.45 (10 mM Hepes, 136 $\mathrm{mM} \mathrm{NaCl}, 2.7 \mathrm{mM} \mathrm{KCl}, 2 \mathrm{mM} \mathrm{MgCl}$, $1 \mathrm{mg} / \mathrm{mL}$ glucose, and $1 \mathrm{mg} / \mathrm{ml} \mathrm{BSA}$ ). Platelet concentrations were adjusted as stated per assay.

\section{Blood collection from animals}

Specific-pathogen-free Btk-KO mice ${ }^{5}$ and littermate controls (wild-types, WT) were housed in the Animal Research Institute Amsterdam facility under standard care. Studies were reviewed and approved by the Central Authority for Scientific Procedures on Animals (CCD) and the Animal Welfare Body (IVD) of the Academic Medical Centre, University of Amsterdam (ID number: 174125-1-80). The animal care and use protocol adhered to the Dutch Experiments on Animals Act (WOD) and European Directive of 22 September 2010 (Directive 2010/63/EU) in addition to the Directive of 6 May 2009 (Directive 2009/41/EC).

Only male mice between 8 and 12 weeks of age were used for experiments. Citrated blood was drawn from WT and Btk-KO mice from the vena cava inferior under full anaesthesia induced by intraperitoneal injection of ketamine (125 mg/kg bodyweight) and dexmedetomidine (300 $\mu \mathrm{g} /$ kg bodyweight). After blood collection mice were sacrificed by severing the diaphragm.

\section{Whole blood thrombus formation under flow}

Thrombus formation under flow with human blood was performed as described 6. Briefly, glass coverslips were coated with 3 microspots of collagen type I (100 $\mu \mathrm{g} / \mathrm{mL})$, collagen type III $(100 \mu \mathrm{g} / \mathrm{mL})$, vWF $(50 \mu \mathrm{g} / \mathrm{mL})$ co-coated with laminin $(100 \mu \mathrm{g} / \mathrm{mL})$, vWF co-coated with rhodocytin $(250 \mu \mathrm{g} / \mathrm{mL})$, vWF co-coated with ristocetin $(250 \mu \mathrm{g} / \mathrm{mL})$, or vWF co-coated with fibrinogen $(250 \mu \mathrm{g} / \mathrm{mL})$, and mounted in a parallel plate flow chamber. Citrated blood samples from healthy donors were incubated with vehicle or $5 \mu \mathrm{M}$ of Btk inhibitor (acalabrutinib, ibrutinib or MK-1026) for 10 minutes at room temperature. Blood samples from CLL patients were used directly. Whole blood from healthy volunteers or CLL patients was recalcified in the presence of PPACK $(40 \mu \mathrm{M})$, and perfused at a wall shear rate of $1000 \mathrm{~s}^{-1}$ for 3.5 minutes. Platelet activation properties were determined by post-perfusion with Hepes buffer (with additional $2 \mathrm{mM} \mathrm{CaCl}$ and $1 \mathrm{U} / \mathrm{mL}$ heparin) containing FITC-conjugated $\alpha$-fibrinogen mAb (1:80), AF647-conjugated CD62-P (1:100), and AF568-conjugated annexin A5 (1:200).

Thrombus formation under flow with mouse blood was performed as described ${ }^{7}$. Glass coverslips were coated with collagen type I $(100 \mu \mathrm{g} / \mathrm{mL})$ and blood samples, in the presence of 
PPACK $(57.1 \mu \mathrm{M})$ and Fragmin $(35.7 \mu \mathrm{M})$, were incubated with $5 \mu \mathrm{M}$ Btk inhibitor (acalabrutinib, ibrutinib or MK-1026) for 10 minutes at $37^{\circ} \mathrm{C}$. Next, the blood samples were recalcified and perfused at a shear rate of $1000 \mathrm{~s}^{-1}$ for 3.5 minutes. To measure platelet activation status, thrombi were post-stained with Tyrode Hepes buffer, pH 7.45 (5 mM Hepes, $136 \mathrm{mM} \mathrm{NaCl}, 2.7 \mathrm{mM} \mathrm{KCl}$, $0.42 \mathrm{mM} \mathrm{NaH}_{2} \mathrm{PO}_{4}, 1 \mathrm{mg} / \mathrm{mL}$ glucose, $1 \mathrm{mg} / \mathrm{ml} \mathrm{BSA}, 2 \mathrm{mM} \mathrm{CaCl}, 2 \mathrm{mM} \mathrm{MgCl}$ and $1 \mathrm{U} / \mathrm{mL}$ heparin) containing PE-labelled Jon-A (1:20) and FITC-conjugated anti-P-selectin mAb (1:40) and AF647labelled Annexin-A5 (1:200).

For both human and mouse experiments, brightfield and fluorescent images were captured using an EVOS microscope (Bothel, WA, USA). Images were analysed using specific scripts in the open-access Fiji software (Laboratory for Optical and Computational Instrumentation, University of Wisconsin-Madison, WI, USA) as described ${ }^{6,8}$. Values for the following parameters of thrombus formation and platelet activation were obtained: morphological score of platelet adhesion and thrombus formation (P1; scale 0-5), surface area coverage of adhered platelets (P2; \%SAC), platelet aggregate contraction score (P3; scale 0-3), platelet aggregate multilayer score (P4; scale $0-3)$, coverage of multi-layered platelet aggregation (P5; \%SAC), integrin $\alpha_{\| 1} \beta_{3}$ activation (P6; \%SAC), P-selectin expression (P7; \%SAC) and PS exposure (P8; \%SAC). For comparative data analysis, cumulative histograms were generated of scaled values from 0-10 for each parameter. For patient data subtraction heatmap representing the effect of ibrutinib treatment in individual patients. Average values obtained with blood of patients without ibrutinib treatment was set at 0 for reference. Effects were filtered for changes greater than the 2x SD range of the different platelet parameters.

\section{Light transmission aggregometry}

Isolated platelets $\left(250 \times 10^{9}\right.$ platelets/L) from healthy volunteers were incubated with vehicle or $3.3 \mu \mathrm{M}$ of Btk inhibitor (acalabrutinib, ibrutinib or MK-1026) for 10 minutes at $37^{\circ} \mathrm{C}$, whereas platelets from CLL patients were used directly after preheating to $37^{\circ} \mathrm{C}$ for 10 minutes.

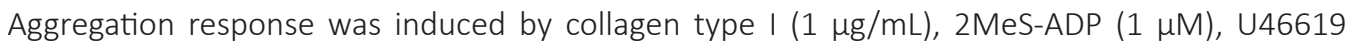
$(1 \mu \mathrm{M})$, rhodocytin $(1 \mu \mathrm{g} / \mathrm{mL})$ or thrombin $(1 \mathrm{nM})$. Platelet aggregation was recorded using a Chronolog optical aggregometer (Havertown PA, USA) and maximum amplitude was quantified at 8 minutes after agonist addition.

\section{Flow cytometry}

Washed platelets $\left(100 \times 10^{9}\right.$ platelets/L) from healthy volunteers or CLL patients were supplemented with $2 \mathrm{mM} \mathrm{CaCl}_{2}$. Samples from healthy volunteers were incubated with vehicle or $1 \mu \mathrm{M}$ of Btk inhibitor (acalabrutinib, ibrutinib or MK-1026) for 10 minutes at room temperature, whereas patient samples were used directly. Platelets were stimulated by CRP-XL $(5 \mu \mathrm{g} / \mathrm{mL}), 2 \mathrm{MeS}$ $\operatorname{ADP}(1 \mu \mathrm{M})$, or thrombin $(1 \mathrm{nM})$ for 15 minutes at RT. Activation of integrin $\alpha_{\| b} \beta_{3}$ and P-selectin 


\section{Chapter 6}

expression were determined using FITC-conjugated PAC1 mAb (1:20) and AF647-conjugated CD62-P mAb (1:40) respectively. For measuring phosphatidyl-serine (PS) exposure platelets were activated with a combination of CRP-XL $(5 \mu \mathrm{g} / \mathrm{mL})$ and thrombin $(4 \mathrm{nM})$ for 1 hour at $37^{\circ} \mathrm{C}$ and labelled with FITC-conjugated annexin A5. Flow cytometry was performed in duplicates using a BD Accuri C6TM flow cytometer and accompanying software (Erembodegem, Belgium).

Platelet activation in whole blood from WT and Btk-KO mice was performed using pooled blood samples $(5 \mu \mathrm{l})$, in the presence of PPACK $(57.1 \mu \mathrm{M})$ and Fragmin $(35.7 \mu \mathrm{M})$, incubated with $5 \mu \mathrm{M}$ Btk inhibitor (acalabrutinib, ibrutinib or MK-1026) for 10 minutes at $37^{\circ} \mathrm{C}$. Platelets were then stimulated for 30 minutes at room temperature with control buffer (PBS), CRP-XL (1 $\mu \mathrm{g} / \mathrm{ml})$, 2MeS-ADP $(1 \mu \mathrm{M})$ or PAR4-AP $(0.1 \mathrm{mg} / \mathrm{ml})$. Platelet activation was assessed by flow cytometry using APC-conjugated anti-CD61, BB700-labelled anti-CD62P, PE-conjugated JON/A and FITClabelled lactadherin. The labelling was terminated by addition of 40 volumes of buffer. Analysis was carried out immediately by flow cytometry using a CytoFLEX (Beckman Coulter, Brea, CA) until 10,000 CD61+ events were recorded. All stimulations and staining were done in duplicate. Compensation settings were validated using single-stained controls. Percentage of positivity was determined using unstimulated controls. Data analysis was done using FlowJoTM v10 (BD Biosciences, San Jose, CA).

\section{Cytosolic $\mathrm{Ca}^{2+}$ measurements}

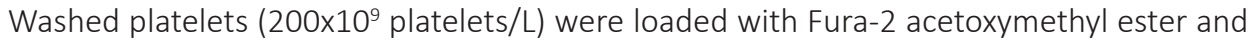
changes in cytosolic $\mathrm{Ca}^{2+}\left(\left[\mathrm{Ca}^{2+}\right]_{\mathrm{i}}\right)$ were measured in 96-well plates using a FlexStation 3 (Molecular Devices, San Jose, CA, USA) as described ${ }^{9,10}$. In brief, platelet suspension was preincubated with vehicle or $1 \mu \mathrm{M}$ Btk inhibitor (acalabrutinib, ibrutinib or MK-1026) for 10 minutes at RT. Fura-2 loaded platelets in presence of $\mathrm{CaCl}_{2}$ were stimulated by CRP-XL $(10 \mu \mathrm{g} / \mathrm{mL})$. Changes in Fura-2 fluorescence were measured in duplicate and ratio values were calculated and are presented as $[\mathrm{Ca}+]_{\mathrm{i}}$ in $\mathrm{nM}$.

\section{PamGene kinase assay}

Washed platelets $\left(500 \times 10^{9}\right.$ platelets/L) were pretreated with vehicle (control) or $1 \mu \mathrm{M}$ ibrutinib, acalabrutinib or MK-1026 for 10 minutes at $37^{\circ} \mathrm{C}$ and were subsequently stimulated with $5 \mu \mathrm{g} / \mathrm{mL} C R P-X \mathrm{~L}$ in the presence of $2 \mathrm{mM} \mathrm{CaCl}_{2}$. Unstimulated, resting platelets served as control. After 90 seconds of stimulation, samples were lysed by adding 1:1 M-PER Mammalian Extraction Buffer containing Halt Phosphatase Inhibitor and EDTA-free Halt Protease Inhibitor Cocktail (1:100 each; Thermo Fischer Scientific). Samples were lysed for 15 minutes on ice and afterwards centrifuged for 15 minutes at $10,000 \mathrm{~g}$ at $4^{\circ} \mathrm{C}$. Supernatants were collected and protein content was quantified with a BioRad DC protein kit (Hercules CA, USA).

Tyrosine kinase profiles were determined using the PamChip ${ }^{\circledR}$ peptide tyrosine kinase 
microarray system on PamStation ${ }^{\circledR} 12$ (PTK; PamGene International, 's-Hertogenbosch, The Netherlands). Each PTK-PamChip ${ }^{\circledR}$ array contains 144 individual phospho-site(s) that are peptide sequences derived from substrates for TKs. Each peptide on the chip builds a 15-amino acid sequence representing a putative endogenous phosphorylation site which functions as a TK substrate. The phosphorylation of the peptides is visualized by detection of the fluorescent signal which is emitted as a result of the binding of the FITC-conjugated PY20 anti-phosphotyrosine antibody.

For the PTK assay, $7.5 \mu \mathrm{g}$ of protein was applied per array ( $\mathrm{N}=4$ per condition) and carried out using the standard protocol supplied by Pamgene. All reagents used for PTK activity profiling were supplied by Pamgene International B.V. Initially, to prepare the PTK Basic Mix, the freshly frozen lysate was added to $4 \mu \mathrm{L}$ of $10 \times$ protein PTK reaction buffer (PK), $0.4 \mu \mathrm{L}$ of $100 \times$ bovine serum albumin (BSA), $0.4 \mu \mathrm{L}$ of $1 \mathrm{M}$ dithiothreitol (DTT) solution, $4 \mu \mathrm{L}$ of $10 \times$ PTK additive, $4 \mu \mathrm{L}$ of $4 \mathrm{mM}$ ATP and $0.6 \mu \mathrm{L}$ of monoclonal anti-phosphotyrosine FITC-conjugate detection antibody (clone PY20). Total volume of the PTK Basic Mix was adjusted to $40 \mu \mathrm{L}$ by adding distilled water $\left(\mathrm{H}_{2} \mathrm{O}\right)$. Before loading the PTK Basic Mix on the array, a blocking step was performed applying $30 \mu \mathrm{L}$ of $2 \%$ BSA to the middle of every array and washing with PTK solution for PamChip ${ }^{\circledR}$ preprocessing. Next, $40 \mu \mathrm{L}$ of PTK Basic Mix were applied to each array of the PamChips ${ }^{\circledR}$. Then, the microarray assays were run for 94 cycles. An image was recorded by a CCD camera PamStation ${ }^{\circledR} 12$ at kinetic read cycles 32-93 at 10, 50 and 200 ms and at end-level read cycle at 10, 20, 50, 100 and 200 ms. The spot intensity at each time point was quantified (and corrected for local background) using the BioNavigator software version 6.3 (PamGene International, 's-Hertogenbosch, The Netherlands). Upstream Kinase Analysis (UKA) ${ }^{11}$, a functional scoring method (PamGene) was used to rank kinases based on combined specificity scores (based on peptides linked to a kinase, derived from 6 databases) and sensitivity scores (based on treatment-control differences).

\section{Statistical analysis}

Data for healthy volunteers are shown as mean \pm standard error of mean (SEM), whereas CLL patients are presented as median \pm interquartile ranges. GraphPad Prism 8.3.0 software (La Jolla, CA, USA) was used for statistical analysis. For healthy donor and CLL patient samples a non-parametric and unpaired t-test (Mann-Whitney) was used to compare vehicle and inhibitor treatment in vitro, whereas for mouse samples statistics were calculated using a one-way non-parametric Anova (Kruskal-Wallis) to compare WT versus Btk-KO samples, as well as the comparison between vehicle and inhibitor treatment in both WT and Btk-KO mice. A p-value less than 0.05 was considered to be statistically significant in which * is $p<0.05, * *$ is $p<0.01$ and $* * *$ is $p<0.001$. 


\section{References}

1. Bergmeier, W, Bouvard, D, Eble, JA, Mokhtari-Nejad, R, Schulte, V, Zirngibl, $H$ et al. Rhodocytin (aggretin) activates platelets lacking alpha(2)beta(1) integrin, glycoprotein $\mathrm{VI}$, and the ligand-binding domain of glycoprotein Ibalpha. J Biol Chem. 2001; 276, 25121-25126.

2. Rodeghiero, F, Tosetto, A, Abshire, T, Arnold, DM, Coller, B, James, P et al. ISTH/SSC bleeding assessment tool: a standardized questionnaire and a proposal for a new bleeding score for inherited bleeding disorders. J Thromb Haemost. 2010; 8, 2063-2065.

3. van der Meijden, PE, Feijge, MA, Giesen, PL, Huijberts, M, van Raak, LP \& Heemskerk, JW. Platelet P2Y12 receptors enhance signalling towards procoagulant activity and thrombin generation. A study with healthy subjects and patients at thrombotic risk. Thromb Haemost. 2005; 93, 1128-1136.

4. Mattheij, NJ, Gilio, K, van Kruchten, R, Jobe, SM, Wieschhaus, AJ, Chishti, AH et al. Dual mechanism of integrin alphallbbeta3 closure in procoagulant platelets. J Biol Chem. 2013; 288, 13325-13336.

5. Hendriks, RW, de Bruijn, MF, Maas, A, Dingjan, GM, Karis, A \& Grosveld, F. Inactivation of Btk by insertion of lacZ reveals defects in B cell development only past the pre-B cell stage. EMBO J. 1996; $15,4862-4872$.

6. de Witt, SM, Swieringa, F, Cavill, R, Lamers, MM, van Kruchten, R, Mastenbroek, T et al. Identification of platelet function defects by multi-parameter assessment of thrombus formation. Nat Commun. 2014; 5, 4257.

7. van Geffen, JP, Swieringa, F, van Kuijk, K, Tullemans, BME, Solari, FA, Peng, B et al. Mild hyperlipidemia in mice aggravates platelet responsiveness in thrombus formation and exploration of platelet proteome and lipidome. Sci Rep. 2020; 10, 21407.

8. van Geffen, JP, Brouns, SLN, Batista, J, McKinney, H, Kempster, C, Nagy, M et al. High-throughput elucidation of thrombus formation reveals sources of platelet function variability. Haematologica. 2019; 104, 1256-1267.

9. Feijge, MA, van Pampus, EC, Lacabaratz-Porret, C, Hamulyàk, K, Levy-Toledano, S, Enouf, J et al. Interindividual variability in $\mathrm{Ca} 2+$ signalling in platelets from healthy volunteers: effects of aspirin and relationship with expression of endomembrane Ca2+-ATPases. Br J Haematol. 1998; 102, 850-859.

10. Jooss, NJ, De Simone, I, Provenzale, I, Fernandez, DI, Brouns, SLN, Farndale, RW et al. Role of Platelet Glycoprotein VI and Tyrosine Kinase Syk in Thrombus Formation on Collagen-Like Surfaces. Int J Mol Sci. 2019; 20. 2788.

11. Chirumamilla, CS, Fazil, M, Perez-Novo, C, Rangarajan, S, de Wijn, R, Ramireddy, P et al. Profiling Activity of Cellular Kinases in Migrating T-Cells. Methods Mol Biol. 2019; 1930, 99-113. 


\section{Supplemental table}

Supplemental Table 6.1. IC $\mathrm{C}_{50}$ values $(\mu \mathrm{M})$ of Btk inhibitors for inhibition of GPVI- and CLEC-2 induced platelet aggregation and activation in presence (PRP) or absence of plasma (WP).

\begin{tabular}{|c|c|c|c|c|c|c|}
\hline \multirow[b]{3}{*}{ Btk inhibitor } & \multicolumn{3}{|c|}{ Aggregation } & \multicolumn{3}{|c|}{ Activation } \\
\hline & \multicolumn{2}{|c|}{ GPVI } & \multirow{2}{*}{$\begin{array}{c}\text { CLEC-2 } \\
\text { WP }\end{array}$} & \multicolumn{2}{|c|}{ GPVI } & \multirow{2}{*}{$\begin{array}{c}\text { GPVI + PAR } \\
\text { PS } \\
\text { exposure }\end{array}$} \\
\hline & PRP & WP & & $\begin{array}{c}\text { Integrin } \\
\text { activation }\end{array}$ & Secretion & \\
\hline Ibrutinib & 3.31 & 0.425 & 0.170 & 0.196 & 0.176 & 0.332 \\
\hline Acalabrutinib & 9.85 & 1.635 & 1.275 & 0.982 & 1.439 & 0.723 \\
\hline MK-1026 & 8.84 & 0.820 & 0.487 & 0.388 & 0.807 & 0.410 \\
\hline
\end{tabular}

\section{Supplemental figures}
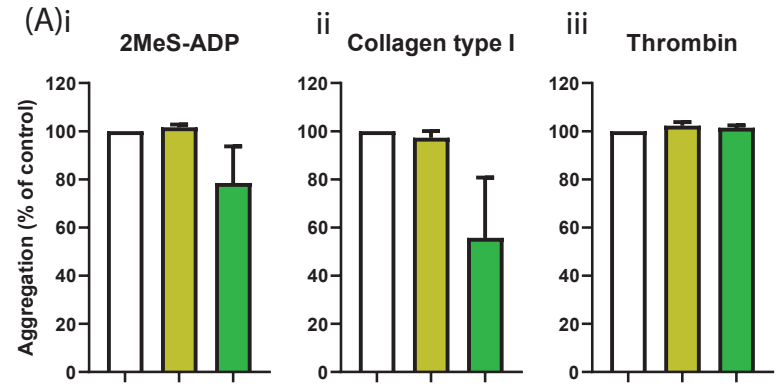

iv

U46619

(B)
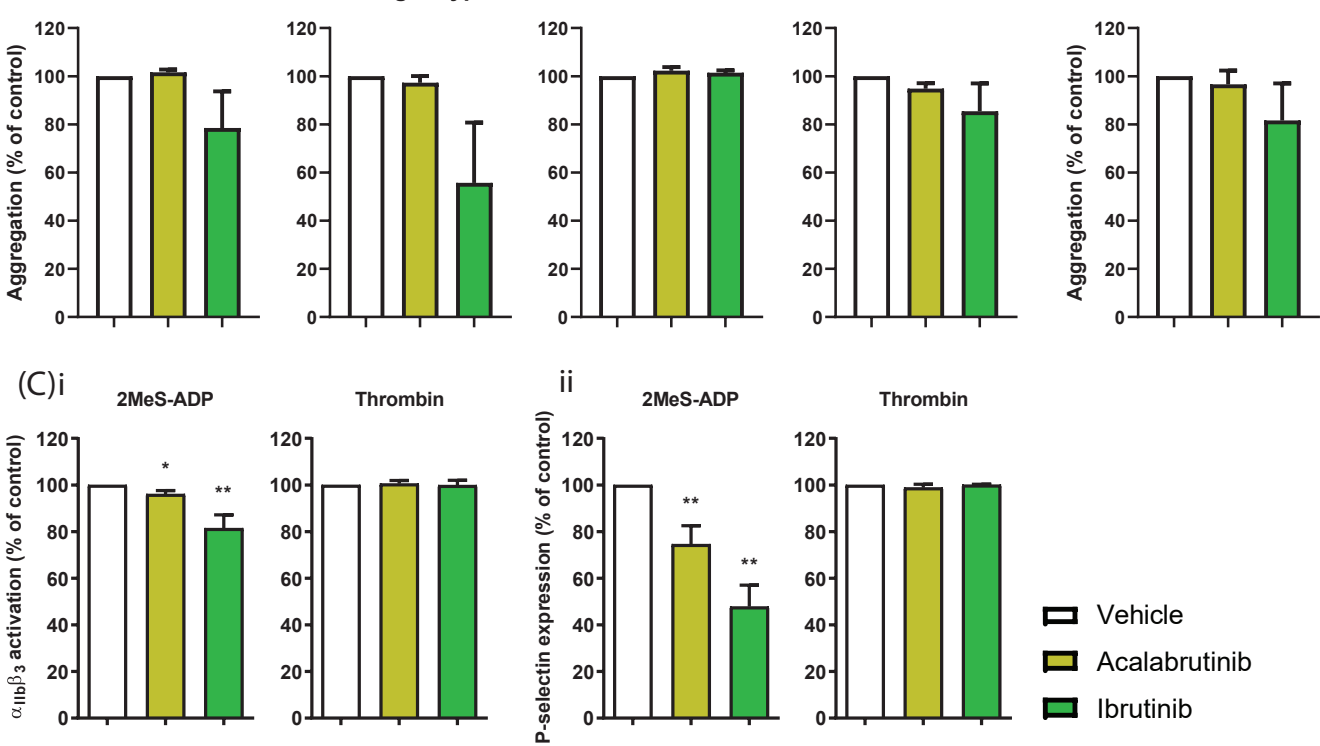

Supplemental Figure 6.1. Platelet aggregation and activation of blood samples from healthy volunteers with ibrutinib and acalabrutinib, as well as dose-response of collagen-induced aggregation. (A) Washed platelets $\left(250 \times 10^{9} / \mathrm{L}\right)$ from healthy volunteers were incubated with vehicle, ibrutinib $(3.3 \mu \mathrm{M})$ or acalabrutinib $(3.3 \mu \mathrm{M})$. Aggregation was induced

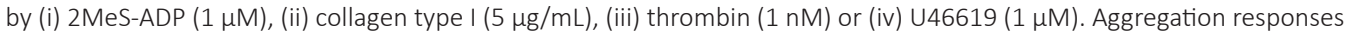
were measured during 8 minutes. (B) PRP $\left(250 \times 10^{9} / \mathrm{L}\right)$ was incubated with vehicle, ibrutinib $(5 \mu \mathrm{M})$ or acalabrutinib $(5$ $\mu \mathrm{M})$ for 10 minutes at $37^{\circ} \mathrm{C}$ and stimulated with ristocetin $(2 \mathrm{mg} / \mathrm{mL})$. Aggregation responses were measured during 8 minutes. (C) Washed platelets $\left(100 \times 10^{9} / \mathrm{L}\right)$ were stimulated with $2 \mathrm{MeS}-\mathrm{ADP}(1 \mu \mathrm{M})$ or thrombin $(1 \mathrm{nM})$ for 15 minutes and analysed by flow cytometry. Shown are percentages of platelets binding FITC-labelled PAC1 mAb against integrin $\alpha_{\| 1 b} \beta_{3}$ and AF647-labelled CD62P mAb for $\alpha$-granule secretion, respectively. Data are shown as means $\pm \operatorname{SEM}(n=5-6) . * p<0.05, * *$ $p<0.01, * * * p<0.001$ vs vehicle. 
(A)i

Collagen type III

ii

vWF + Laminin

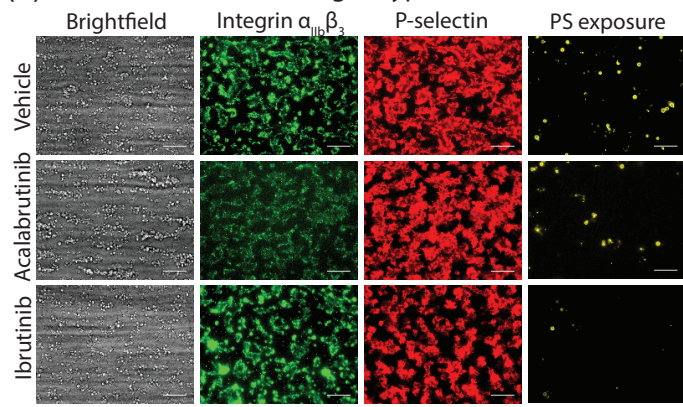

Brightfield Integrin $\alpha_{\mu b} \beta_{3}$

P-selectin

PS exposure
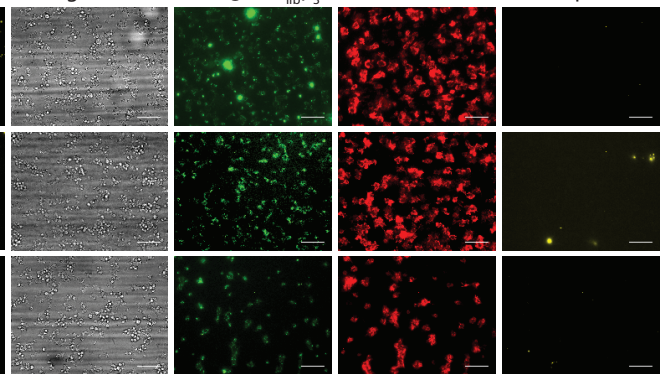

iii

VWF + Ristocetin

iv

$v W F+$ Fibrinogen

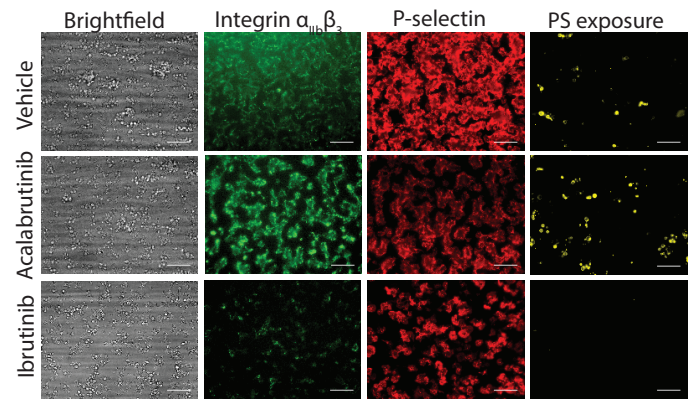

Brightfield

Integrin $\alpha_{\| b} \beta_{3}$

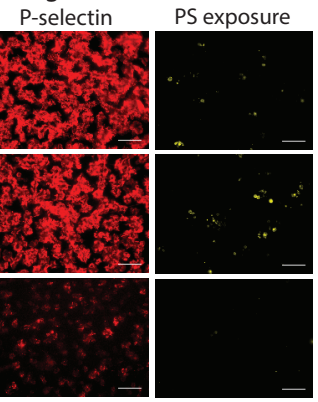

(B) $\mathrm{i}$
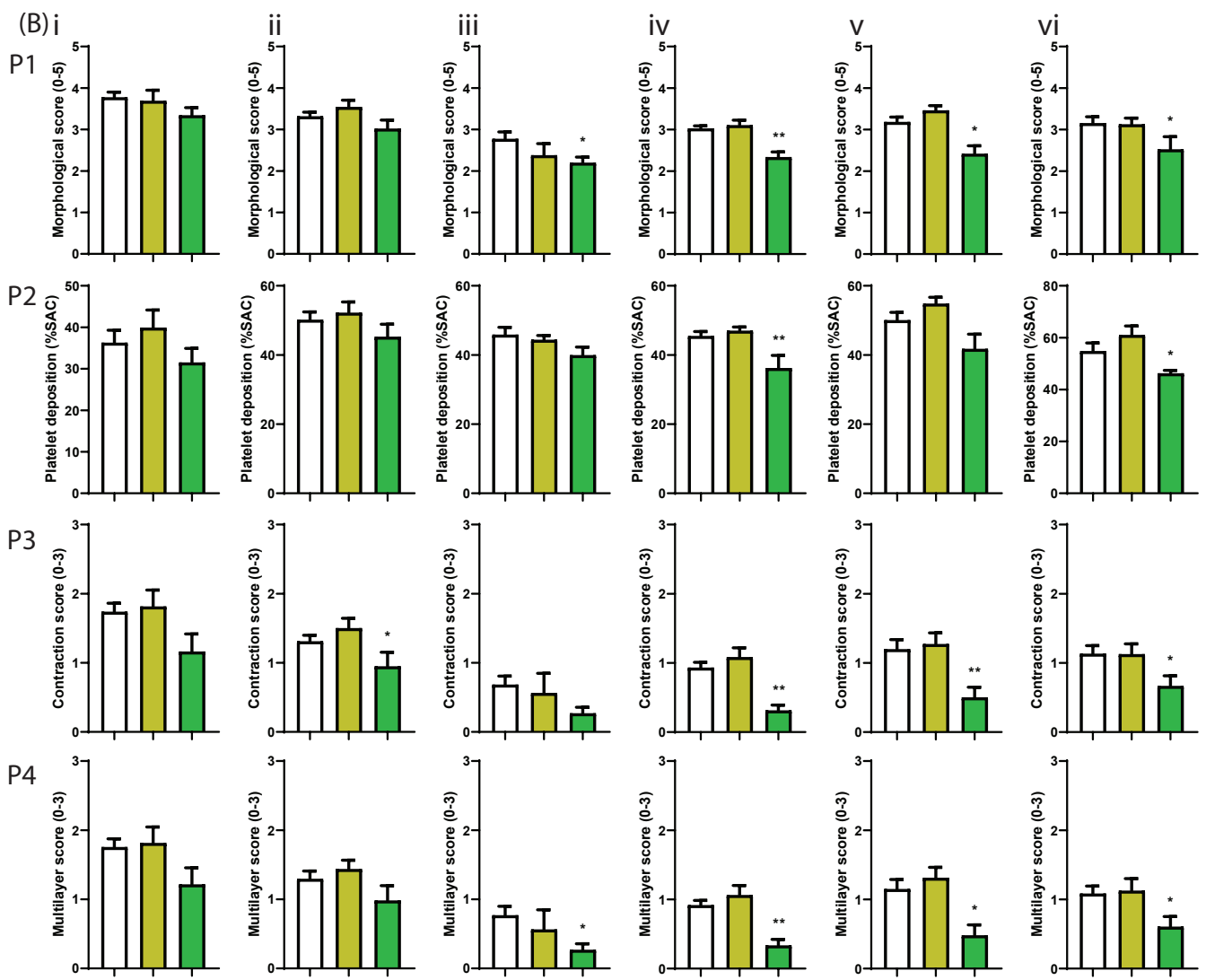
(B)i

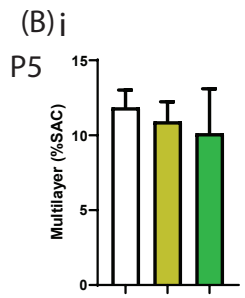

P6

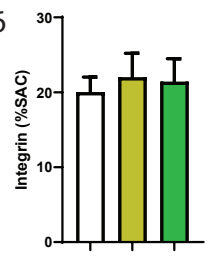

P7
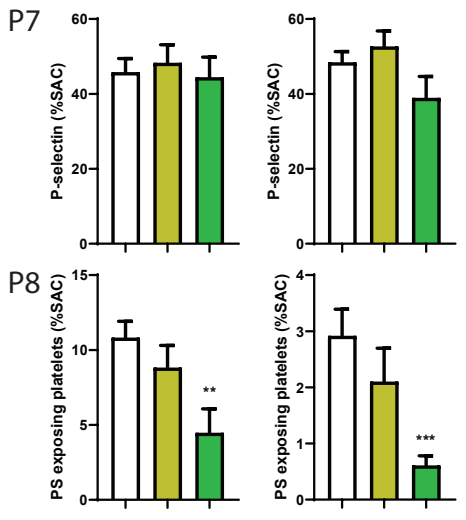
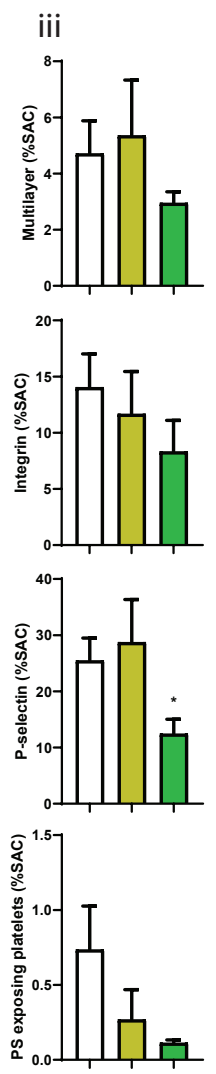
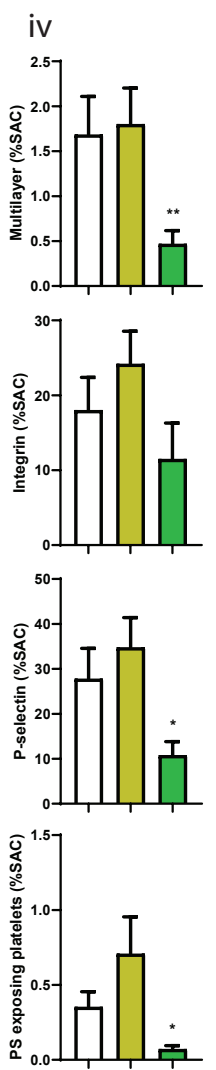
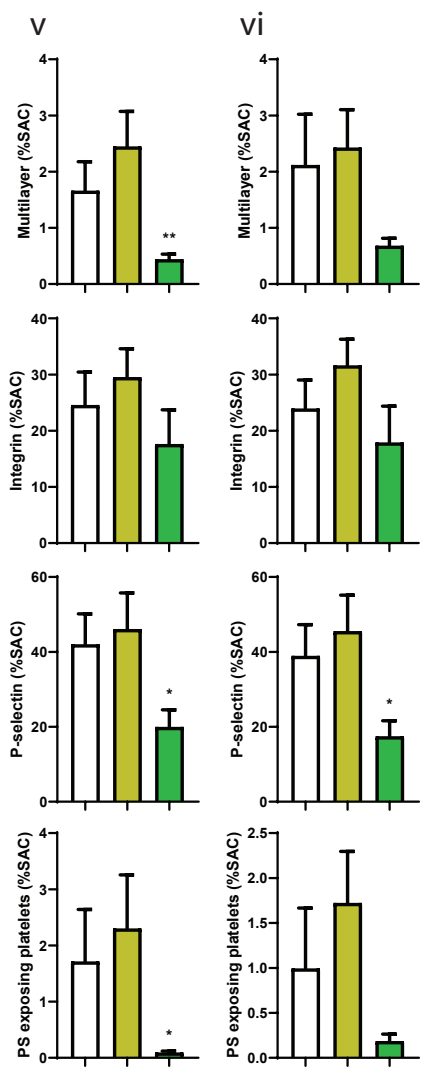

\section{口 Vehicle \\ 口 Acalabrutinib \\ 口 Ibrutinib}

Supplemental Figure 6.2. Thrombus formation under flow with blood from healthy donors in presence of acalabrutinib or ibrutinib over multiple microspots. (A) Representative brightfield and fluorescence images and (B) quantification of control (vehicle, white bars), acalabrutinib (5 $\mu \mathrm{M}$, grey bars) or ibrutinib (5 $\mu \mathrm{M}$, black bars) over (i) collagen type I, (ii) collagen type III, (iii) VWF co-coated with rhodocytin, (iv) VWF co-coated with laminin, (v) VWF co-coated with ristocetin and (vi) VWF co-coated with fibrinogen. P1 = morphological score of platelet adhesion and thrombus formation, $\mathrm{P} 2=$ surface area coverage of adhered platelets, P3 = platelet aggregate contraction score, $\mathrm{P} 4=$ platelet aggregate multilayer score, P5 = coverage of multi-layered platelet aggregation, P6 = integrin $\alpha_{110} \beta_{3}$ activation, P7 = P-selectin expression and P8 $=$ PS exposure. Data are shown as means \pm SEM $(n=6-8) .{ }^{*} p<0.05,{ }^{* *} p<0.01,{ }^{* * *} p<0.001$. 

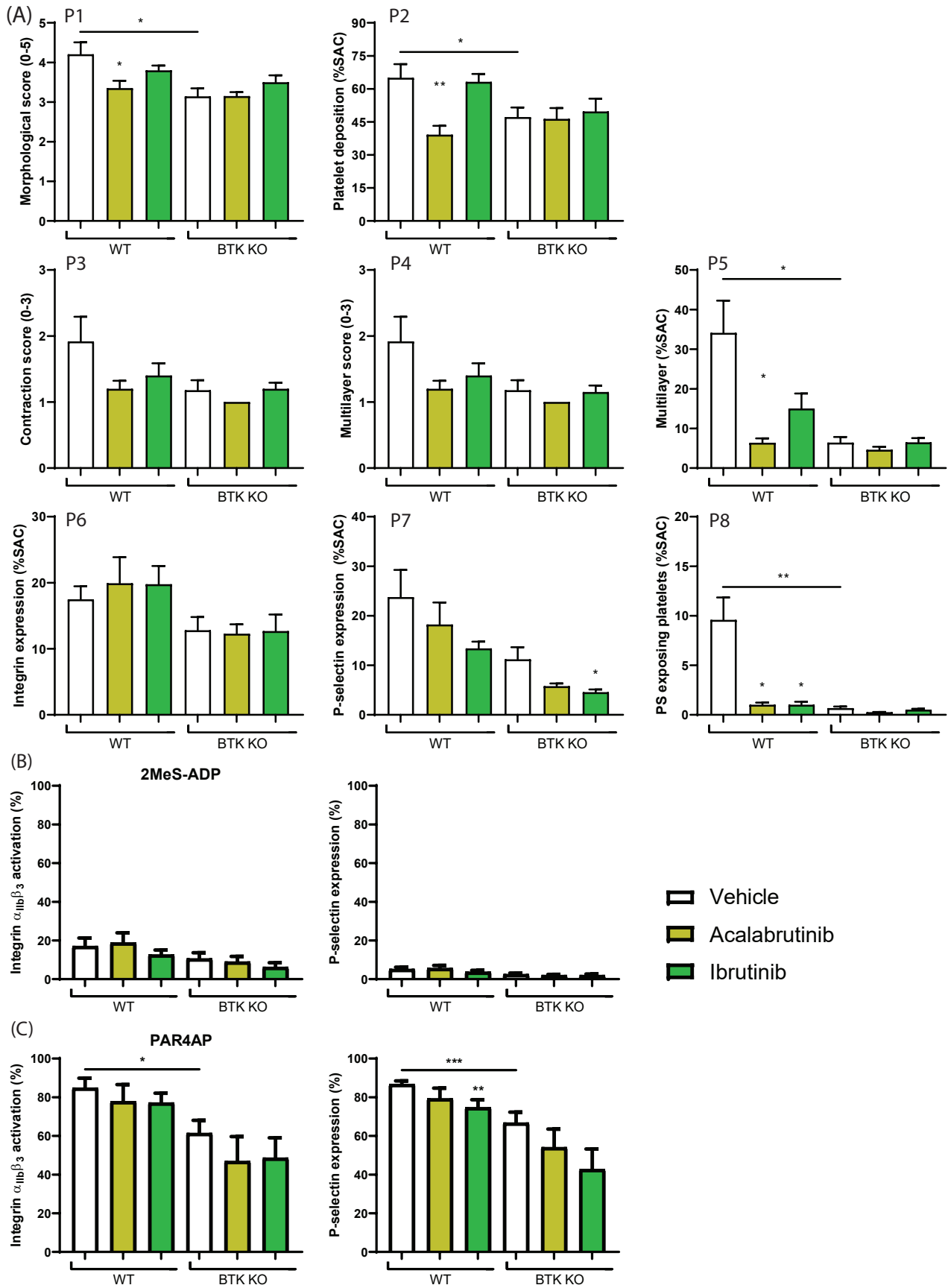

Supplemental Figure 6.3. Thrombus formation and platelet activation with blood of WT or Btk-KO mice. Citrated whole blood from WT or Btk-KO mice pre-incubated with control (vehicle, white bars), acalabrutinib (5 $\mu \mathrm{M}$, grey bars) or ibrutinib ( $5 \mu \mathrm{M}$, black bars) was perfused for 3.5 minutes at a wall shear rate of 1,000 s-1 over a collagen type I surface. (A) Quantification of brightfield and fluorescence images. (B-C) Platelet activation in whole blood induced by (B) 2MeS-ADP $(1 \mu \mathrm{M})$ or $(\mathrm{C})$ PAR4AP $(100 \mu \mathrm{g} / \mathrm{mL})$. Histograms show the percentages of platelets binding PE-labelled JON/A monoclonal antibody or PerCP-labelled CD62P mAb in WT and Btk-KO mice. Data are presented as means \pm SEM $(n=5-6), * p<0.05, * *$ $p<0.01$ vs vehicle or indicated otherwise. 
(A) $\mathrm{i}$ 2MeS-ADP ii Collagen iii Thrombin iv U46619
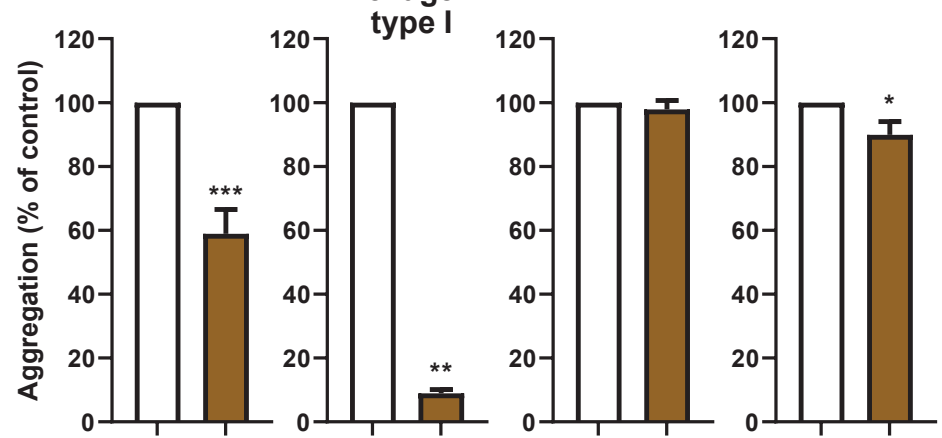

(B) Ristocetin

(C)

2MeS-ADP

Thrombin
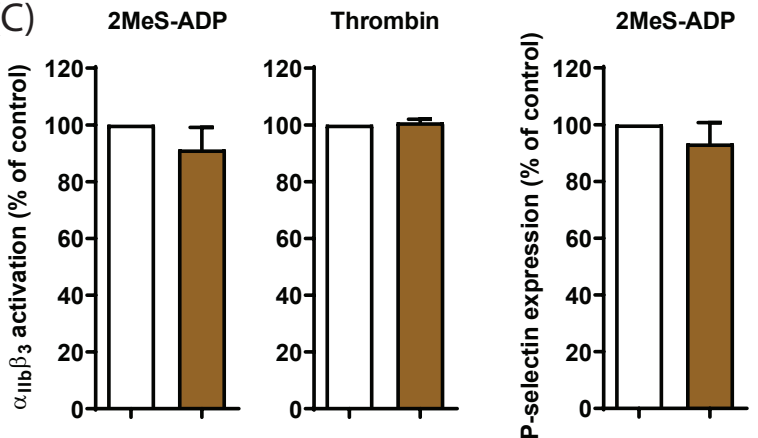

Thrombin

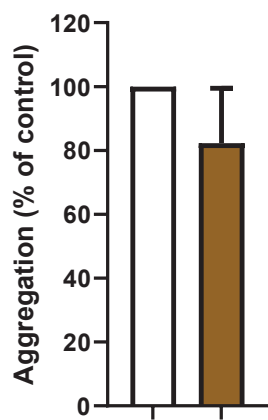

Supplemental Figure 6.4. Platelet aggregation and activation of blood samples from healthy volunteers in presence of MK-1026. (A) Washed platelets $\left(250 \times 10^{9} / \mathrm{L}\right)$ from healthy volunteers were incubated with vehicle or MK-1026 (3.3 $\mu \mathrm{M})$. Aggregation was induced by (i) 2MeS-ADP (1 $\mu \mathrm{M})$, (ii) collagen type I (5 $\mathrm{gg} / \mathrm{mL}$ ), (iii) thrombin (1 nM) or (iv) U46619 $(1 \mu \mathrm{M})$. Aggregation responses were measured during 8 minutes. (B) PRP $\left(250 \times 10^{9} / \mathrm{L}\right)$ from healthy volunteers were incubated with vehicle or MK-1026 $(5 \mu \mathrm{M})$ for 10 minutes at $37^{\circ} \mathrm{C}$ and stimulated with ristocetin $(2 \mathrm{mg} / \mathrm{mL})$. Aggregation responses were measured during 8 minutes. (C) Washed platelets $\left(100 \times 10^{9} / \mathrm{L}\right)$ were stimulated with $2 \mathrm{MeS}-\mathrm{ADP}(1 \mu \mathrm{M})$ or thrombin $(1 \mathrm{nM})$ for 15 minutes and analysed by flow cytometry. Shown are percentages of platelets binding FITClabelled PAC1 mAb against integrin $\alpha_{\| 1} \beta_{3}$ and AF647-labelled CD62P mAb for $\alpha$-granule secretion, respectively. Data are represented as means $\pm \operatorname{SEM}(n=5-6), * p<0.05$. 

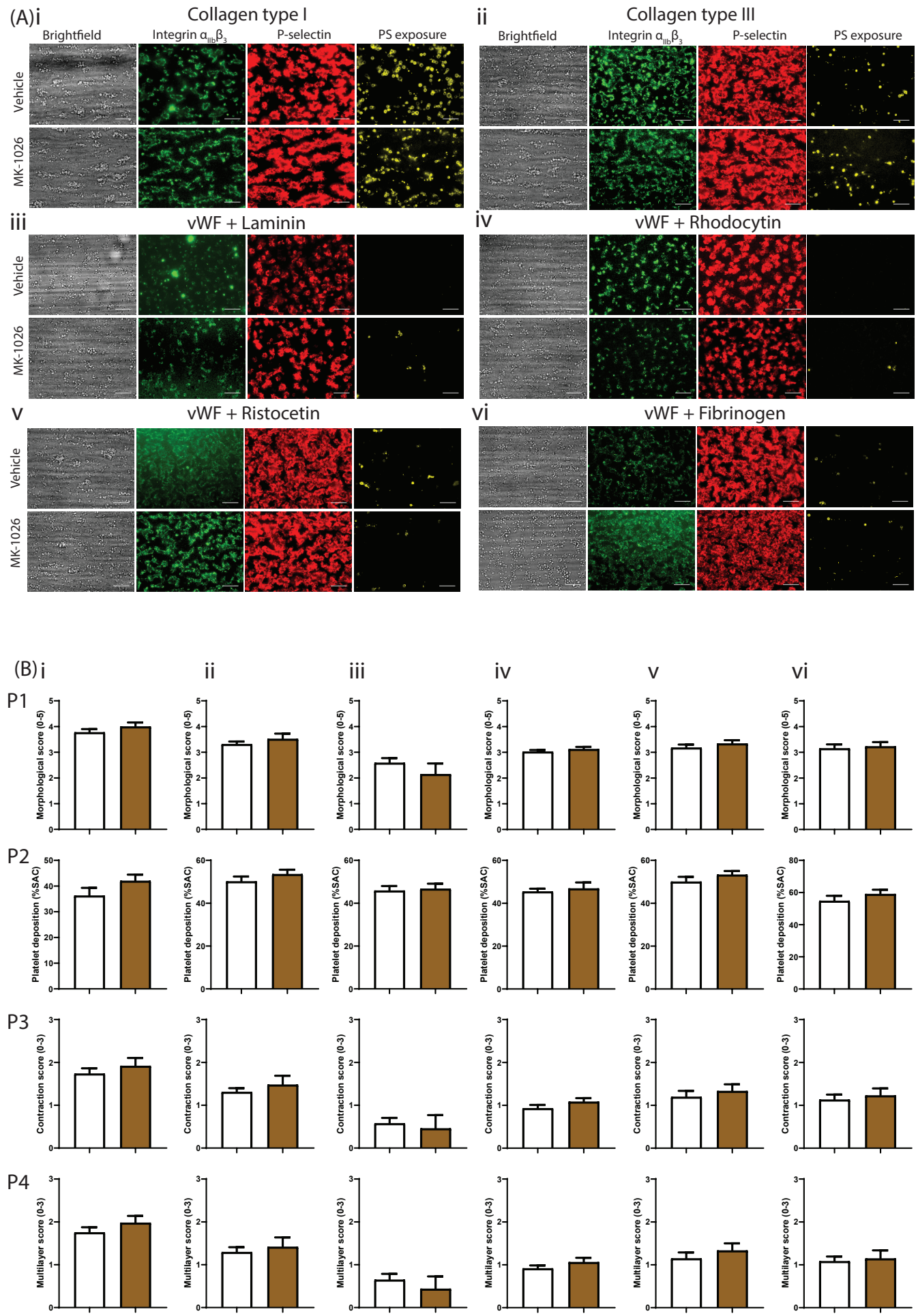
(B) $\mathrm{i}$
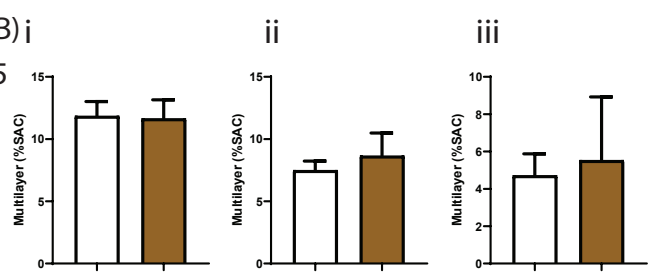

iv

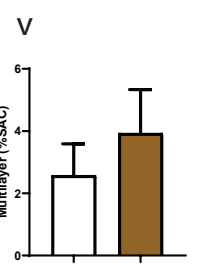

vi

P6
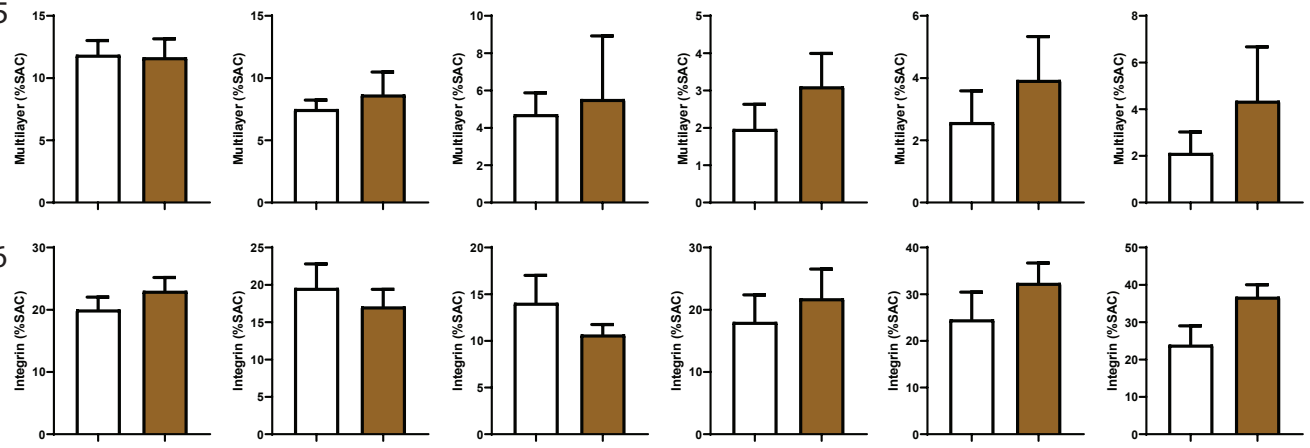

P7
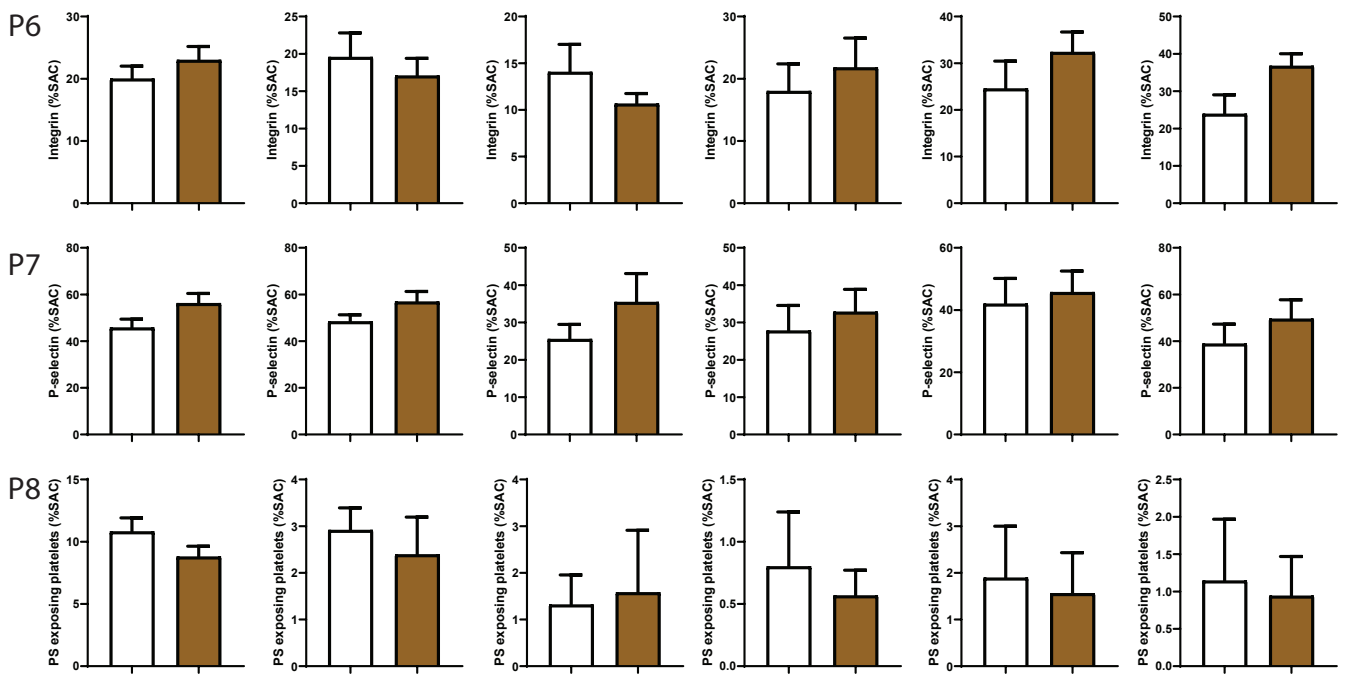

Supplemental Figure 6.5. Thrombus formation under flow with blood from healthy volunteers in presence of MK-1026 over multiple microspots. (A) Representative brightfield and fluorescence images and (B) quantification of control (white bars), MK-1026 (sand-coloured bars) over (i) collagen type I, (ii) collagen type III, (iii) VWF co-coated with laminin, (iv) vWF co-coated with rhodocytin, (v) VWF co-coated with ristocetin and (vi) VWF co-coated with fibrinogen. Data are shown as means $\pm \operatorname{SEM}(n=6)$. 
(A)
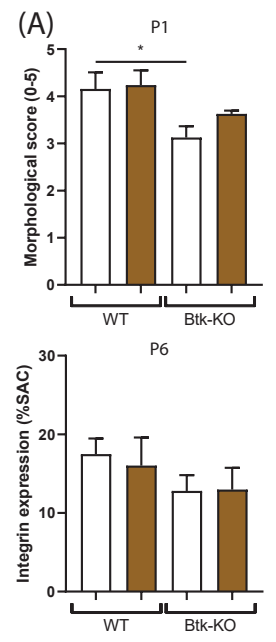

(B)

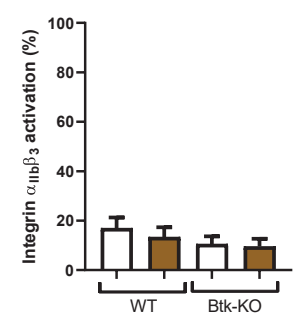

P2
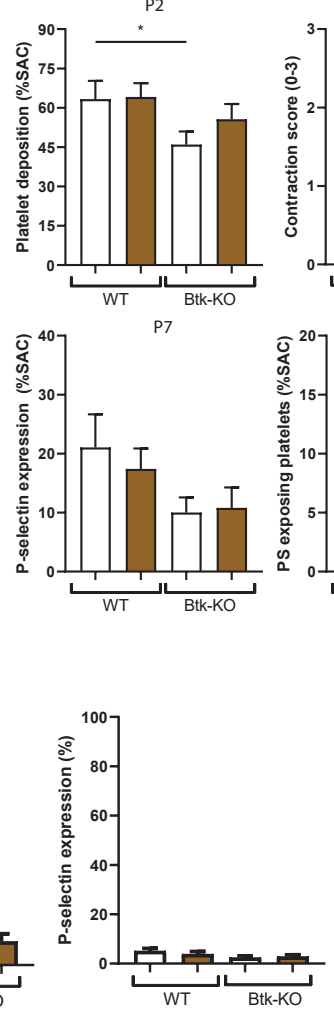

P3
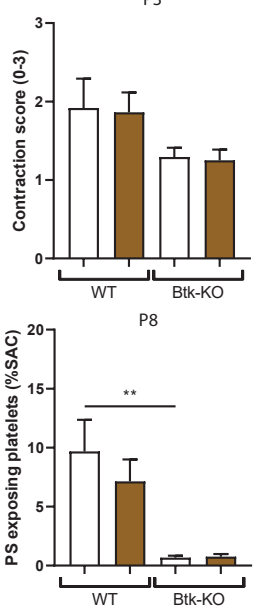

P4

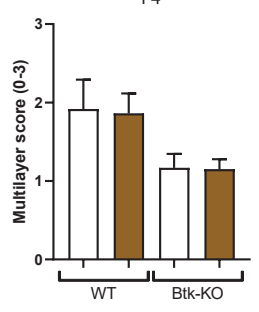

口 Vehicle

口 MK-1026

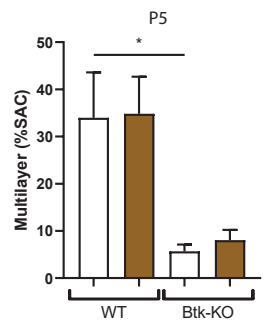

(C) PAR4AP
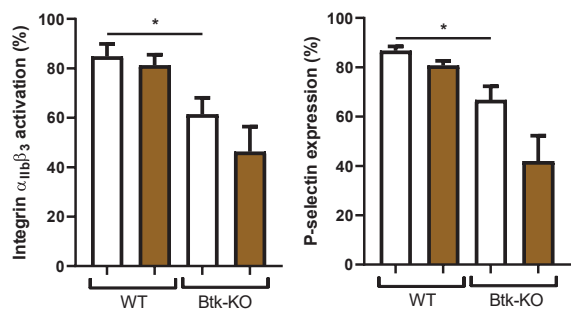

Supplemental Figure 6.6. Thrombus formation under flow with blood of WT or Btk-KO mice over collagen type I surface. (A) Quantification of brightfield and fluorescence images of control (white bars) or MK-1026 (sand-coloured bars) over collagen type I. Parameter 1-8 are as described for Supplement Figure 6.2. (B-C) Platelet activation in whole blood induced by (B) 2MeS-ADP $(1 \mu \mathrm{M})$ or (C) PAR4AP $(100 \mu \mathrm{g} / \mathrm{mL})$. Histograms show the percentages of platelets binding PElabelled JON/A monoclonal antibody or PerCP-labelled CD62P mAb in WT and Btk-KO mice. Data are presented as means $\pm \operatorname{SEM}(n=5-6), * p<0.05, * * p<0.01$ vs vehicle or indicated otherwise. 

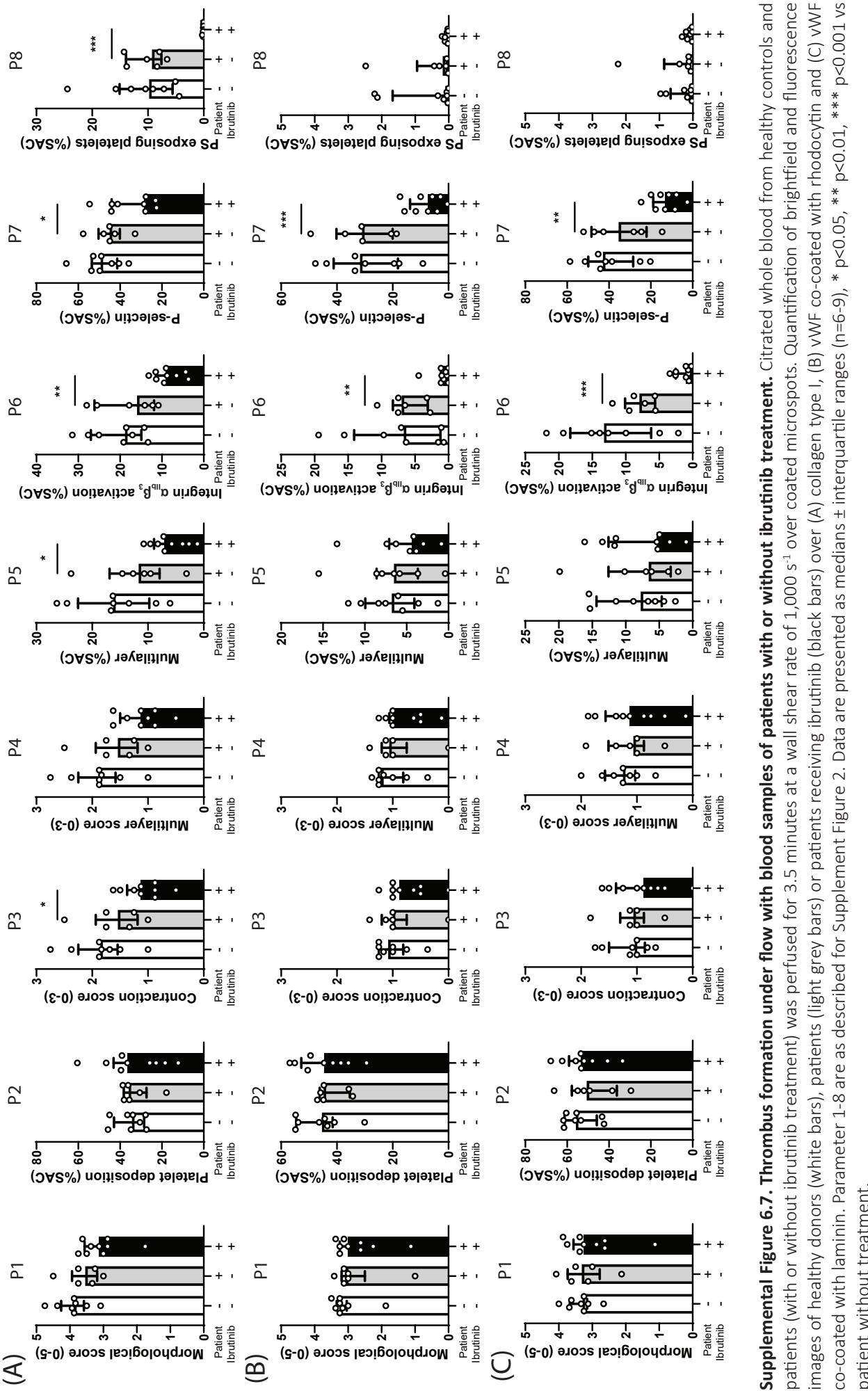
(A)

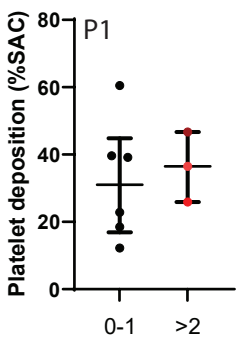

ISTH BAT score

(B)

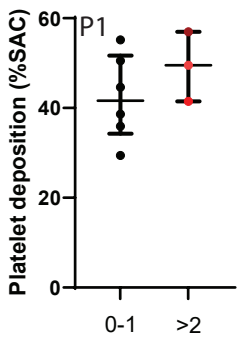

ISTH BAT score

(C)

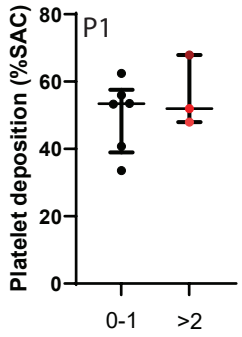

ISTH BAT score

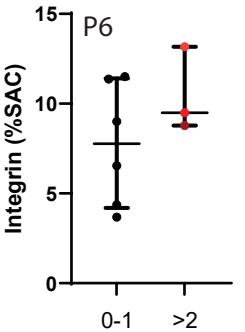

ISTH BAT score

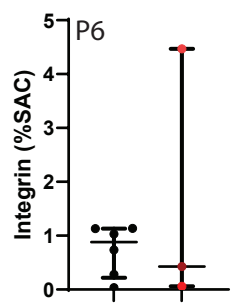

0-1 >2
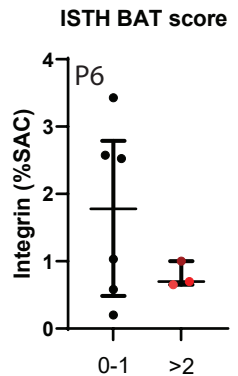

ISTH BAT score

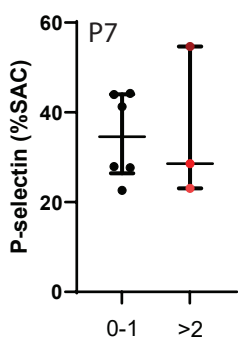

ISTH BAT score

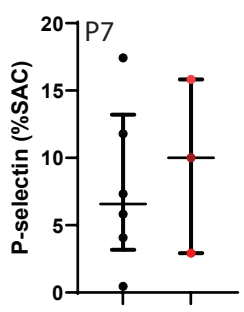

$0-1>2$

ISTH BAT score

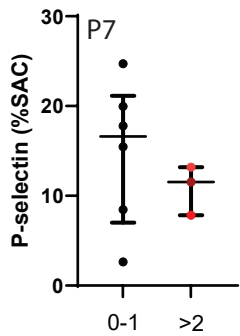

ISTH BAT score

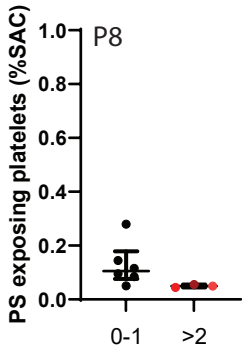

ISTH BAT score

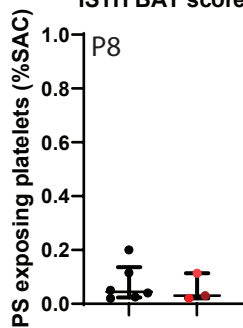

$0-1>2$

ISTH BAT score

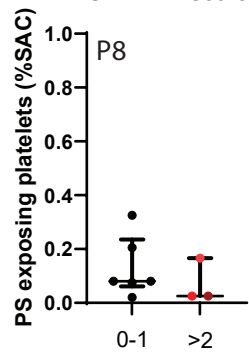

ISTH BAT score

(D) Collagen $(1 \mu \mathrm{g} / \mathrm{ml})$ Rhodocytin $(1 \mu \mathrm{g} / \mathrm{ml})$ ADP $(1 \mu \mathrm{M})$

$\mathrm{U} 46619(1 \mu \mathrm{M})$ Thrombin (1 nM)

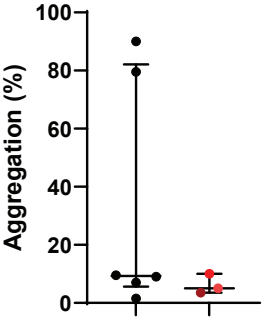

0-1 >2

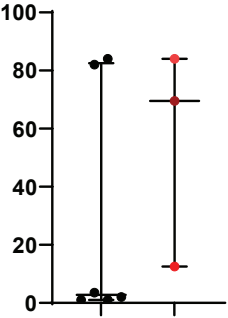

0-1 >2

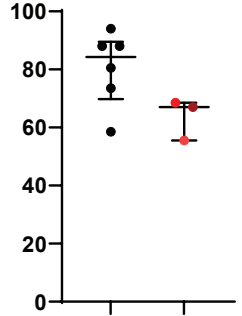

0-1 >2

ISTH BAT score

ISTH BAT score

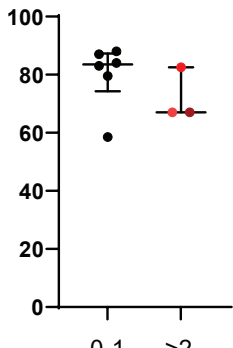

ISTH BAT score

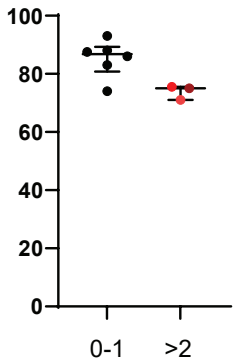

ISTH BAT score

Supplemental Figure 6.8. Thrombus formation and aggregation with blood samples of ibrutinib-treated patients with or without bleeding. Citrated whole blood from patients receiving ibrutinib treatment was perfused for 3.5 minutes at a wall shear rate of $1,000 \mathrm{~s}^{-1}$ over coated microspots. Quantification of brightfield and fluorescence images of ibrutinib-treated patients with a ISTH BAT score of 0-1 (black dots) or a ISTH BAT score over a value of 2 (red dots) over (A) collagen type I, (B) VWF co-coated with rhodocytin and (C) VWF co-coated with laminin. Parameters 1, 6-8 are as described for Supplement Figure 6.2. (D) Light transmission aggregometry was induced in isolated platelets (250x10\% L) by collagen $(1 \mu \mathrm{g} / \mathrm{mL})$, rhodocytin $(1 \mu \mathrm{g} / \mathrm{mL}), 2 M e S-A D P(1 \mu \mathrm{M}), \mathrm{U} 46619(1 \mu \mathrm{M})$ or thrombin $(1 \mathrm{nM})$. Scatter plots show the percentage of aggregation of patients receiving ibrutinib with a low bleeding score (0-1, black dots) or a bleeding score of 2 or higher (red dots). Each dot represents one individual patient. Data are presented as medians \pm interquartile ranges $(n=3-6)$. 
6 

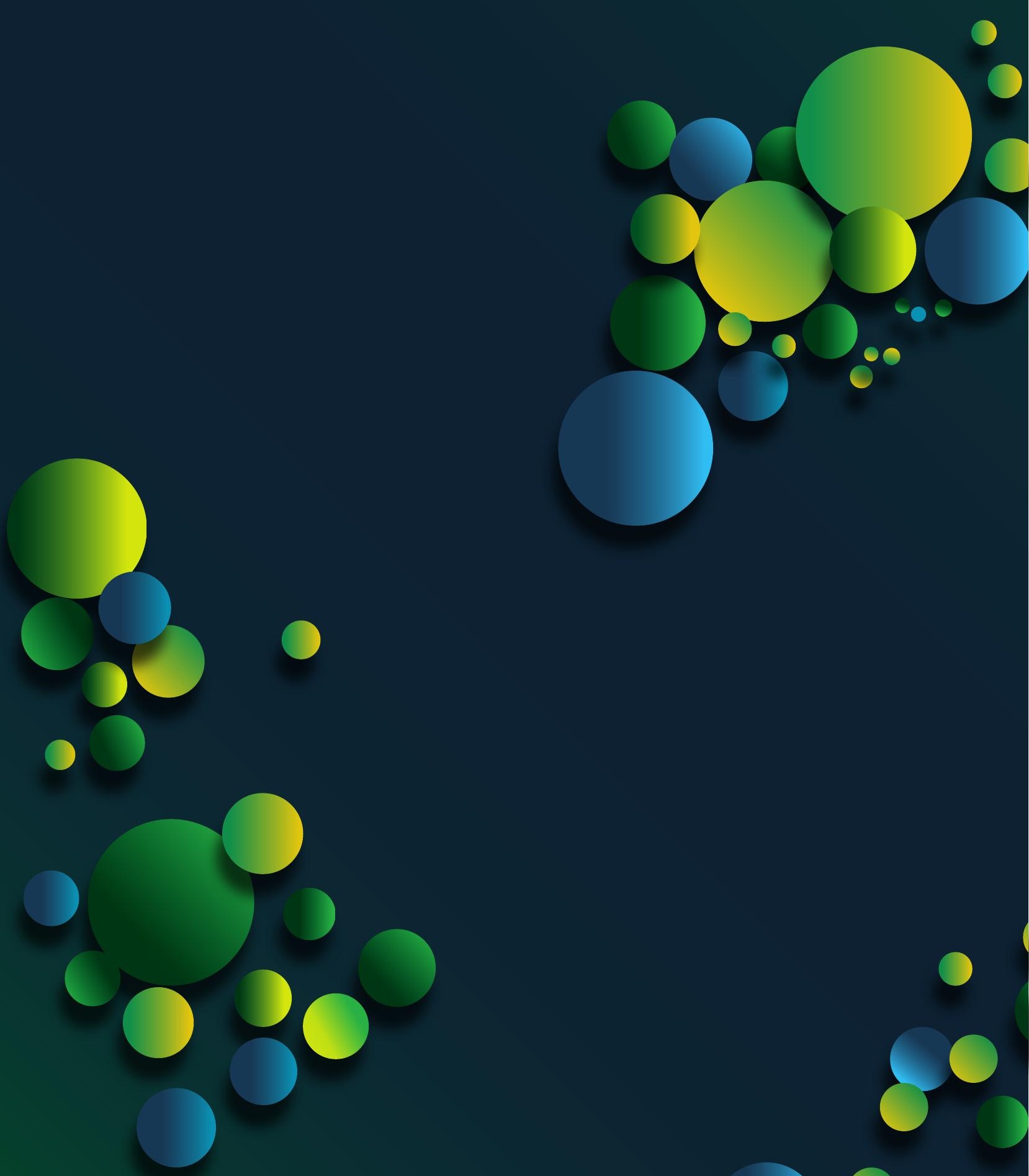


\section{Chapter 7}

Multiparameter screening for plateletinhibitory effects of tyrosine kinase inhibitors used for cancer treatment

Tullemans BME, Veninga A, Fernandez DI, Aarts MJB, Eble JA, van der Meijden PEJ, Heemskerk JWM and Kuijpers MJE

In revision 


\section{Abstract}

Background: Currently antiplatelet drugs, used for treatment of arterial thrombosis, often coincide with an increased risk of bleeding. Hence, platelet inhibitors that preserve haemostasis are warranted. Multiple tyrosine kinase inhibitors (TKIs), used for cancer treatment, have been reported to inhibit platelet function, with minor bleeding symptoms. In this study, we investigated the antiplatelet properties of several clinically used TKIs as exploration of possible repurposing these compounds as antiplatelet drugs.

Methods: Eight TKIs were selected based on the affinity for tyrosine kinases expressed in platelets and limited bleeding symptoms. Whole blood samples, platelet-rich plasma (PRP) or isolated platelets from healthy donors were pre-incubated with the TKI or vehicle. Platelet aggregation, activation, intracellular calcium mobilisation and whole blood thrombus formation under flow were determined.

Results: Overall, the TKIs with the highest affinities for platelet-expressed targets strongly inhibited platelet functions. Dasatinib and sunitinib dose-dependently reduced collagen-induced aggregation in PRP and washed platelets, while pazopanib, cabozantinib and vatalanib inhibited this response in washed platelets only, and fostamatinib, axitinib and lapatinib showed no or limited effects. Fostamatinib effectively reduced whole blood thrombus formation under flow on collagen, as well as other substrates. Pazopanib, sunitinib, dasatinib, axitinib and vatalanib mildly reduced thrombus formation on collagen. Intracellular calcium measurements and activation responses in isolated platelets were inhibited by dasatinib, fostamatinib, sunitinib and pazopanib. After glycoprotein-VI receptor-stimulation fostamatinib, cabozantinib and vatalanib decreased the highly activated platelet population, whereas negative, resting populations were increased.

Conclusions: Strong inhibitory effects of dasatinib, fostamatinib, sunitinib and pazopanib pointed to interference in early collagen receptor-induced signalling events, when compared to cabozantinib and vatalanib. Since Fostamatinib, sunitinib, pazopanib and vatalanib are reported to cause no more than mild bleeding symptoms, these are promising candidates to explore further as antiplatelet drugs. 


\section{Introduction}

Ischaemic heart disease and stroke accounted >15 million deaths worldwide in 2019, making arterial thrombosis a leading global cause of death ${ }^{1}$. The major underlying cause are the thrombotic complications of atherosclerosis, either via plaque rupture or erosion ${ }^{2,3}$. Current treatment is mostly (dual) antiplatelet therapy, such as aspirin and clopidogrel that prevent thromboxane $A_{2}$ release and ADP receptor activation, respectively ${ }^{4}$. However, these inhibitors mostly have an irreversible mode of action on platelet G-protein coupled receptor (GPCRs) signalling pathways and coincide with an increased risk of bleeding ${ }^{5}$. Therefore, novel antiplatelet drugs are being developed that inhibit thrombosis, but do not interfere in haemostasis 5 . In this respect glycoprotein (GP)VI and C-type lectin-like receptor 2 (CLEC-2) receptors, as well as downstream tyrosine kinases, have been postulated as interesting antithrombotic targets ${ }^{6}$.

GPVI is an immunoreceptor tyrosine-based activation motif (ITAM)-linked receptor, activated by collagen and fibrin(ogen), resulting in initial platelet activation and subsequent thrombus formation ${ }^{7-9}$. Deficiency of GPVI in mice suppressed arterial thrombosis without substantial prolongation of bleeding time ${ }^{10,11}$. Inhibition of GPVI has also been reported to protect from thrombosis upon plaque rupture in mice ${ }^{12,13}$. Furthermore, patients with defects in GPVI have only a mild bleeding diathesis ${ }^{14}$ or remain asymptomatic ${ }^{15}$. Two GPVI inhibitors showing promising antithrombotic effects, are currently being tested in phase II clinical trials: the Fab fragment ACT017 and Revacept, a collagen binding dimeric GPVI-Fc fusion protein ${ }^{6}$. However, a disadvantage of these treatments is the administration via intravenous injections.

CLEC-2 is a hemITAM-linked receptor, activated by the endogenous ligand podoplanin ${ }^{16}$. CLEC-2 deficient mice are protected from arterial thrombus formation and displayed only a minor effect on haemostasis ${ }^{17,18}$. In contrast, deficiency of both GPVI and CLEC-2 resulted in lack of cessation of tail bleeding, as well as profound reduction of arterial thrombosis ${ }^{19}$. In human blood, a monoclonal antibody (mAb) to CLEC-2 has been shown to block CLEC-2 induced platelet activation in vitro ${ }^{20}$. However, no clinical trials targeting CLEC-2 have started until now.

Both GPVI and CLEC-2 heavily depend on tyrosine kinase signalling including Syk, Btk and Src family kinases (SFK) ${ }^{21}$. In addition, other receptor signalling pathways, likely to a lesser extent, use include tyrosine kinases, such as those evoked by GPIb, integrin $\alpha_{110} \beta_{3}$, AxI and Tie, which are receptors for VWF, fibrinogen, Gas6 and angiopoietin, respectively ${ }^{22,23}$. With such a wide mode of action on platelet activation, while leaving GPCR signalling mostly intact, tyrosine kinase inhibitors (TKIS) may provide an alternative way to inhibit platelet activation ${ }^{6}$. During the past decades, many orally available TKIs have been developed for cancer treatment ${ }^{24}$, 25, with different inhibitory profiles against tyrosine kinases (Table 7.1). These compounds have been shown to be well tolerated, and in general cause only a mild increase in risk of bleeding. The Syk inhibitor fostamatinib, used for treatment of chronic immune thrombocytopenic purpura (ITP), has been reported to reduce GPVI- and CLEC-2 stimulated platelet aggregation, with only 
minor effects on bleeding time in mice ${ }^{26,27}$. Notably, TKIs targeting a broad spectrum of tyrosine kinases including the receptors for vascular endothelial growth factor (VEGFR), platelet-derived growth factor (PDGFR) and Kit are used for the treatment of renal cell carcinoma. These include sunitinib, pazopanib, axitinib and cabozantinib (Table 7.1). We have recently shown that sunitinib and pazopanib inhibit collagen-induced platelet function both in vitro and in patients receiving treatment ${ }^{28,29}$. Axitinib and cabozantinib also have targets that are present in platelets ${ }^{24}$ and have been associated with increased risk of bleeding ${ }^{30}$, but no effects on platelet function have been reported. On the contrary, several TKIs used for treatment of breast and colon cancer have limited affinity for tyrosine kinases present in platelets, without reported bleeding ${ }^{24}$. These include lapatinib and vatalanib (Table 7.1).

The aim of the present study was to systematically evaluate the antiplatelet properties of eight clinically used TKIs, and to assess their potential for repurposing of these compounds as antiplatelet drugs. Therefore, we investigated the effects of these compounds on platelet function in isolated platelets and in whole blood under flow, using different agonists to include multiple signalling pathways.

\section{Materials and Methods}

\section{Materials}

The active metabolite of fostamatinib (R406) was obtained from InvivoGen (San Diego CA, USA). Active metabolites of axitinib (A-1107), cabozantinib, dasatinib and pazopanib were

Table 7.1: Characterization of TKIs used for cancer treatment with targets present in platelets.

\begin{tabular}{|c|c|c|c|c|c|}
\hline TKI & Reported Targets & $\begin{array}{l}\text { Targets in } \\
\text { platelets (MKs) }\end{array}$ & Used for treatment of & $\begin{array}{l}\text { Bleeding } \\
\text { reported }\end{array}$ & Ref \\
\hline Dasatinib & $\begin{array}{l}\text { PDGFR, EFGR, BCR-ABL, } \\
\text { EphA2, Kit, SFK }\end{array}$ & $\begin{array}{l}\text { SFK: Src, Fyn, Lck, Lyn, } \\
\text { Yes, Btk, (Kit) }\end{array}$ & CP CML, AP MB, LB CML & Yes & 66,67 \\
\hline Fostamatinib & Syk & $\begin{array}{l}\text { Syk, Src, Fgr, Fyn, Ick, } \\
\text { Lyn, Yes }\end{array}$ & B-cell lymphoma, CLL & No & 54 \\
\hline Sunitinib & $\begin{array}{l}\text { VEGFR, PDGFR, CSF-R, } \\
\text { Ret, Kit, Flt3 }\end{array}$ & (Ret, Kit) & RCC, GIST, PNET & Yes & $30,66,68,69$ \\
\hline Pazopanib & $\begin{array}{l}\text { VEGFR, PDGFR, FGFR, } \\
\text { Kit, Fms, Itk, Lck }\end{array}$ & Lck (Kit) & NSCLC, OC, RCC, STS, TC & Yes & 30,66 \\
\hline Axitinib & VEGFR, PDGFR $\beta$ & Lck, Yes, Axl, Tie & RCC & Yes & 30,66 \\
\hline Cabozantinib & $\begin{array}{l}\text { VEGFR, Met, Ret, Kit, } \\
\text { Flt3, Axl, Tie }\end{array}$ & Axl, Tie (Ret, Kit) & TC, RCC & Yes & 30,70 \\
\hline Lapatinib & EGFR, ErbB1-2 & ND & $\mathrm{ErbB}^{+} \mathrm{HR}^{-}$or $\mathrm{HR}^{+} \mathrm{BC}$ & No & 66,71 \\
\hline Vatalanib & VEGFR, PDGFR $\beta$, Kit & (Kit) & MCRC & No & 72 \\
\hline
\end{tabular}

AP: acute-phase; BC: breast cancer; CLL: chronic lymphocytic leukaemia; CML: chronic myeloid leukaemia; CP: chronicphase; CSF-R: colony-stimulating factor receptor; EGFR: epidermal growth factor receptor; ErbB2: human epithelial growth factor 2; FGFR: fibroblast growth factor receptor; GIST: gastrointestinal stromal tumour; HR: hormone receptor; LB: Iymphoid blast; MB: myeloid blast; MCRC: metastatic colorectal cancer; MKs, megakaryocytes; ND: not determined; NSCLC: non-small-cell lung cancer; OC: ovarian cancer; PDGFR: platelet-derived growth factor receptor; PNET: primitive neuroectodermal tumour; RCC: renal cell carcinoma; SFK: Src family kinase; STS: soft tissue sarcoma; TC: thyroid cancer; VEGFR: vascular endothelial growth factor receptor. 
purchased from LC Laboratories (Woburn MA, USA). The active metabolite of lapatinib (GW572016) was purchased from Selleckchem (Houston TX, USA), whereas the active metabolite of sunitinib came from Pfizer (New York NY, USA). The active metabolite of vatalanib (PTK787) was obtained from Adooq Bioscience (Irvine CA, USA). Bovine serum albumin (BSA), D(+)glucose, unfractionated heparin and apyrase were purchased from Sigma-Aldrich (Saint Louis MO, USA). Horm collagen type I came from Takeda (Hoofddorp, the Netherlands), whereas the agonists collagen-related peptide crosslinked (CRP-XL) and von Willebrand factor III (vWFIII) were obtained from CambCol Laboratories (Cambridge, UK). Rhodocytin was purified from Calloselasma rhodostoma venom as described previously ${ }^{31}$. Thrombin was from Enzyme Research Laboratories Inc (South Bend, IN, USA). U46619 (thromboxane $A_{2}$ receptor agonist) came from Cayman Chemicals (Ann Arbor MI, USA). 2-Methylthio-adenosine-diphosphate (2MeS-ADP) and D-phenylalanyl-prolyl-arginyl chloromethyl ketone (PPACK) were obtained from Santa Cruz Biotechnology (Dallas TX, USA). Laminin was purchased from Octapharma (Lachen, Switzerland), whereas Fura-2-AM and allophycocyanin (APC)-labelled mouse anti-human CD63 monoclonal antibody (mAb, clone MEM-259) were obtained from Invitrogen, Fisher Scientific (Carlsbad CA, USA). Fluorescein isothiocyanate (FITC)-labelled PAC1 mAb against activated human integrin $\alpha_{\| 1} \beta_{3}$ (340507), Phycoerythrin (PE)-labelled annexin A5, Brilliant Violet (BV)510-conjugated mouse antihuman CD42b mAb (clone HIP1) and BV421-labelled rat anti-human TLT-1 mAb (clone 268420), were purchased from BD Bioscience (Franklin Lakes NJ, USA). FITC-conjugated $\alpha$-fibrinogen mAb was purchased from DAKO (F0111; Santa Clara CA, USA). Alexa Fluor (AF)568-conjugated annexin A5 was purchased from Molecular Probes, Life Technologies (New York NY, USA). Peridininchlorophyll-protein cyanine5.5 (PerCP Cy5.5)-conjugated mouse anti-human CD62-P mAb (clone AK4) and AF647-labelled mouse anti-human CD62-P mAb (clone AK4) were obtained from Biolegend (London, UK). FITC-conjugated annexin A5 was from Pharmatarget (Maastricht, the Netherlands).

\section{Blood collection and platelet isolation}

In accordance with the declaration of Helsinki and approval of the local medical ethical committee (Maastricht University Medical Centre+, MUMC+), we obtained full informed consent from all participants. Blood from healthy volunteers was collected in 3.2\% trisodium citrate tubes by venipuncture, after discarding the first $3 \mathrm{~mL}$ of blood. Blood cell counts and haematological parameters were assessed, using a Sysmex XP300 (Kobe, Japan).

Platelet-rich plasma (PRP) or platelets were isolated from whole blood as described previously ${ }^{32,33}$. Washed platelets were resuspended in Hepes buffer, pH 7.45 (10 mM Hepes, 136 $\mathrm{mM} \mathrm{NaCl}, 2.7 \mathrm{mM} \mathrm{KCl}, 2 \mathrm{mM} \mathrm{MgCl}, 1 \mathrm{mg} / \mathrm{mL}$ glucose, and $1 \mathrm{mg} / \mathrm{mL}$ bovine serum albumin). Platelet count was adjusted as stated per assay. 


\section{Thrombus formation}

Thrombus formation under flow was determined as described ${ }^{34}$. Briefly, glass coverslips were coated with 3 microspots of collagen type I $(100 \mu \mathrm{g} / \mathrm{mL})$, collagen type III $(100 \mu \mathrm{g} / \mathrm{mL})$, vWF $(50 \mu \mathrm{g} / \mathrm{mL})$ co-coated with laminin $(100 \mu \mathrm{g} / \mathrm{mL})$ or vWF co-coated with rhodocytin $(250 \mu \mathrm{g} / \mathrm{mL})$, VWF co-coated with ristocetin $(250 \mu \mathrm{g} / \mathrm{mL})$, or vWF co-coated with fibrinogen $(250 \mu \mathrm{g} / \mathrm{mL})$ and mounted in a parallel plate flow chamber. Citrated blood samples were incubated with the TKIs axitinib, cabozantinib, dasatinib, fostamatinib, lapatinib, pazopanib, sunitinib, vatalanib or vehicle for 10 minutes at room temperature. Pre-incubated whole blood samples were recalcified in presence of PPACK $(40 \mu \mathrm{M})$ and perfused at a wall shear rate of $1000 \mathrm{~s}^{-1}$ for 3.5 minutes. Platelet activation properties were determined by post-perfusion with Hepes buffer (supplemented with $2 \mathrm{mM} \mathrm{CaCl}_{2}$ and $1 \mathrm{U} / \mathrm{mL}$ heparin) containing FITC-conjugated $\alpha$-fibrinogen mAb (1:80), AF647conjugated $\alpha$ CD62P mAb (1:100), and AF568-conjugated annexin A5 (1:200). Brightfield and fluorescence images were captured using an EVOS microscope (Bothel WA, USA).

\section{Analysis of microscopic data}

Microscopic images were analysed using specific scripts in the open-access Fiji software (Laboratory for Optical and Computational Instrumentation, University of Wisconsin-Madison, WI, USA) as described ${ }^{34,35}$. Multiple thrombus formation parameters were obtained from brightfield images including P1: morphological score of platelet adhesion and thrombus formation (scale 0-5), P2: surface area coverage of adhered platelet (\% SAC), P3: platelet aggregate contraction score (scale 0-3), P4: platelet aggregate multilayer score (scale 0-3) and P5: coverage of multilayered platelet aggregation (\% SAC). From fluorescence images, platelet activation parameters were obtained by determining P6: integrin $\alpha_{\| 1} \beta_{3}$ activation (\% SAC), P7: P-selectin expression (\% SAC) and P8: phosphatidylserine (PS) exposure (\% SAC). Heatmaps were generated from the mean values per parameter for each surface for comparative data analysis. Values were scaled from 0-10 based on the highest value of each parameter for each surface. For visualization of the effects, subtraction heatmaps were generated by subtraction of scaled control data from each TKI condition and heatmaps were filtered based on significant differences $(p<0.05)$. Heatmaps were generated using R Core Team (i386 3.2.5; Vienna, Austria).

\section{Platelet aggregation by light transmission aggregometry}

Isolated platelets or PRP $\left(250 \times 10^{9}\right.$ platelets/L) were incubated with TKIs or vehicle for 10 minutes at $37^{\circ} \mathrm{C}$. Maximal aggregation was induced by collagen type I $(1 \mu \mathrm{g} / \mathrm{mL}), 2 \mathrm{MeS}-\mathrm{ADP}$ $(1 \mu \mathrm{M}), \mathrm{U} 46619(1 \mu \mathrm{M})$, rhodocytin $(1 \mu \mathrm{g} / \mathrm{mL})$ or thrombin $(1 \mathrm{nM})$. Platelet aggregation was recorded using a Chronolog optical aggregometer (Havertown PA, USA). The maximum amplitude was quantified at 8 minutes after agonist addition. 


\section{Cytosolic $\mathrm{Ca}^{2+}$ measurements}

Washed platelets $\left(200 \times 10^{9}\right.$ platelets/L) were loaded with Fura-2-AM and changes in cytosolic $\mathrm{Ca}^{2+}\left(\left[\mathrm{Ca}^{2+}\right]_{\mathrm{i}}\right)$ were measured in 96-well plates using a FlexStation 3 (Molecular Devices, San Jose, CA, USA), as described ${ }^{36,37}$. Briefly, platelets in suspension were preincubated with TKIs or vehicle for 10 minutes at room temperature. Pre-loaded and-incubated samples were stimulated by CRP-XL $(10 \mu \mathrm{g} / \mathrm{mL})$. Changes in Fura-2 fluorescence were measured in duplicate, and ratio values were calculated. Data are presented as $\left[\mathrm{Ca}^{2+}\right]_{\mathrm{i}}$ in $\mathrm{nM}$ or area under the curve $(A \cup C)$ in $n M s$.

\section{Platelet activation by flow cytometry}

Washed platelets $\left(25 \times 10^{9}\right.$ platelets/L) were incubated with TKIs or vehicle for 10 minutes at room temperature. Platelets were stimulated, in the presence of $2 \mathrm{mM} \mathrm{CaCl}_{2}$, by CRP-XL (5 $\mu \mathrm{g} / \mathrm{mL})$, 2MeS-ADP $(2 \mu \mathrm{M})$, or thrombin (4 $\mathrm{nM})$ for 15 minutes, and simultaneously stained for multiple activation markers using an antibody mix consisting of FITC-conjugated PAC1 (1:20), BV510-conjugated aCD42b (1:50), BV421-conjugated aTLT1 (1:50), APC-conjugated aCD63 (1:20), PerCP-Cy5.5-conjugated aCD62P (1:500) mAbs. For measuring phosphatidyl-serine (PS) exposure, platelets were activated with a combination of CRP-XL $(5 \mu \mathrm{g} / \mathrm{mL})$ and thrombin (4 $\mathrm{nM}$ ) for 1 hour at $37^{\circ} \mathrm{C}$, and post-labelled with PE-conjugated annexin A5. Samples for activation markers as well as PS exposure were fixed after stimulation for at least 30 minutes at $4^{\circ} \mathrm{C}$ in the dark using a $0.3 \%$ fixation buffer, before measuring 10,000 events per sample on a BD FACSCanto II (BD bioscience, Franklin Lakes, NJ, USA).

\section{Analysis of flow cytometric data}

Separate FCS files were opened in FlowJo V10 software (Treestar, Ashland OR, USA) and checked for data anomalies by FlowAl ${ }^{38}$. Before concatenation, event count per sample was set at 5,000 events by DownSample for equal weighing of every sample. Resulting file was subjected to t-distributed stochastic neighbour embedding (tSNE) analysis to reduce the high dimensionality to a $2 \mathrm{D}$ plot. FlowSOM analysis was used to identify five clusters ${ }^{39}$. Fractions of platelets present in the resulting FlowSOM populations were determined per original sample. Each platelet population was characterised by the expression level of the different markers.

\section{Statistical analysis}

Data are shown as mean \pm standard error of mean (SEM). GraphPad Prism 8.3.0 software (La Jolla, CA, USA) was used for statistical analysis with an unpaired non-parametric t-test (MannWhitney) or one-way ANOVA. A p-value less than 0.05 was considered to be statistically significant, in which $*$ is $p<0.05, * *$ is $p<0.01$ and $* * *$ is $p<0.001$. 


\section{Results}

\section{Characteristics of selected TKIs}

For this study, eight reversible multi-target TKIs were selected based on published affinities for tyrosine kinases (TKs) expressed in platelets, and reported minor bleeding risk (Table 7.1, Figure 7.1) $)^{24}$. Dasatinib and fostamatinib display high affinity for platelet-expressed Syk and Srcfamily kinases (SFKs), while sunitinib, pazopanib, axitinib and cabozantinib show moderate to mild affinity for only some of these platelet-expressed targets (Figure 7.1). Dasatinib and fostamatinib are known as inhibitors of the GPVI, CLEC-2 and $\alpha_{\| 1} \beta_{3}$ activation pathways in platelets ${ }^{26,40}$, but have not been systematically evaluated. Lapatinib and vatalanib have unknown affinities for platelet-expressed kinases (Figure 7.1, Table 7.1).

\section{Distinct effects of selected TKIs on collagen-induced platelet aggregation in the presence or absence of plasma}

Impaired platelet function by TKIs is described to be due to inhibition of in particular the ITAM-linked receptor GPVI 24,25 . Therefore, we first investigated the effects of the active metabolites of different TKIs on collagen-induced aggregation responses. Dose-response curves with isolated platelets showed distinct effects of the different TKIs (Figure 7.2, dotted lines). The most potent aggregation inhibition was observed with dasatinib (>90\% inhibition with $1 \mu \mathrm{M}$, Figure 7.2A). Surprisingly, the combined SFK and Syk-inhibitor fostamatinib, with several targets in platelets, did not show a relevant inhibitory effect (Figure 7.2B). For the weaker SFK inhibitors sunitinib, pazopanib, and cabozantinib, aggregation in washed platelets became nearly abolished with 33 $\mu \mathrm{M}$ (Figure 7.2C-D, F) Unexpectedly, vatalanib without assumed targets in platelets also inhibited this response at $33 \mu \mathrm{M}$ (Figure $7.2 \mathrm{H}$ ). I $\mathrm{IC}_{50}$ values of these four TKIs were between 6-13 $\mu \mathrm{M}$ and a

\begin{tabular}{|c|c|c|c|c|c|c|c|c|c|c|c|c|c|c|c|c|c|}
\hline \multirow[b]{2}{*}{ TKI } & \multicolumn{6}{|c|}{ SFK } & \multicolumn{4}{|c|}{ TFK } & \multicolumn{3}{|c|}{ Others } & \multirow{2}{*}{\multicolumn{2}{|c|}{ Interaction strength }} & \multirow[b]{2}{*}{$\mathrm{Kd}(\mathrm{nM})$} & \multirow[b]{2}{*}{ IC50 (nM) } \\
\hline & $\begin{array}{l}u \\
\text { N }\end{array}$ & 市 & 妾 & 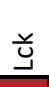 & 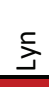 & $\stackrel{\mathscr{\nu}}{\nu}$ & 并 & 盖 & $\stackrel{U}{E}$ & 交 & 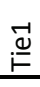 & $\begin{array}{l}\vec{y} \\
\underset{\Sigma}{\Sigma}\end{array}$ & $\frac{\stackrel{D}{u}}{\sum}$ & & & & \\
\hline Dasatinib & +++ & +++ & \pm & +++ & +++ & +++ & \pm & +++ & \pm & - & - & \pm & \pm & +++ & Very strong & $<0.01$ & $<0.1$ \\
\hline Fostamatinib & +++ & ++ & +++ & ++ & ++ & ++ & +++ & + & \pm & + & + & + & + & & Strong & $<0.1$ & $<1$ \\
\hline Sunitinib & \pm & \pm & + & + & + & + & - & + & - & ++ & + & + & + & \pm & Mild & $<1$ & $<10$ \\
\hline & \pm & + & + & + & + & + & + & - & - & - & + & - & - & - & No / ND & $>10$ & ND \\
\hline Pazopanid & - & - & - & - & - & 1 & 1 & 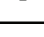 & 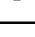 & 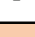 & $\perp$ & 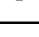 & 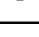 & & & & \\
\hline Axitinib & - & \pm & - & \pm & - & \pm & - & - & - & + & + & - & - & & & & \\
\hline Cabozantinib & - & - & - & - & - & - & - & - & - & + & \pm & - & - & & & & \\
\hline Lapatinib & - & - & - & - & - & - & - & - & - & - & - & - & - & & & & \\
\hline Vatalanib & - & - & - & - & - & - & - & - & - & - & - & - & - & & & & \\
\hline
\end{tabular}

Figure 7.1. Affinity profile of common tyrosine kinase inhibitors (TKIs) for relevant tyrosine kinases implicated in platelet activation. Shown is a heatmap of the affinity-based dissociation constants $(K d)^{62-64}$ or $I C_{50}$ values ${ }^{65}$ of the indicated TKIs. Values are from binding experiments with indicated purified kinases, including Src family kinases (SFK), Syk, Tec family kinases (TFK), focal adhesion kinases (FAK) and MEK. Colour coding with highest affinity (lowest Kd or $\mathrm{IC}_{50}$ ) shown in deep red and lowest affinity (highest $\mathrm{Kd}$ or $\mathrm{IC}_{50}$ ) shown in white. Data are based on ref ${ }^{24}$. 
concentration of $10 \mu \mathrm{M}$ was used for further experiments (Table 7.2). Axitinib and lapatinib with limited or no TK targets slightly affected the aggregation response to collagen at the highest dose tested of $100 \mu \mathrm{M}$ (Figure 7.2E, G).

As most TKIs are protein-bound in plasma, dose response curves were also determined in platelet-rich plasma (PRP; Figure 7.2, solid lines). Upon collagen stimulation, the strongest inhibitory effect in PRP was also observed with dasatinib $\left(\mathrm{IC}_{50}\right.$ of $0.4 \mu \mathrm{M}$, and $>90 \%$ inhibition at $3.3 \mu \mathrm{M}$, Figure 7.2A). In patient studies, plasma concentrations up to $0.1 \mu \mathrm{M}$ dasatinib are reported ${ }^{41}$, which concentration thus was used in following experiments (Table 7.2). Inhibition with fostamatinib, also resulted in a small but significant decrease of the aggregation response ( $\pm 20 \%$ inhibition, Figure $7.2 B)$. For sunitinib, significant inhibition $(60 \%)$ was reached at $33 \mu \mathrm{M}$, which was higher than required in washed platelets (Figure 7.2C). The IC $C_{50}$ value of $33 \mu \mathrm{M}$ sunitinib

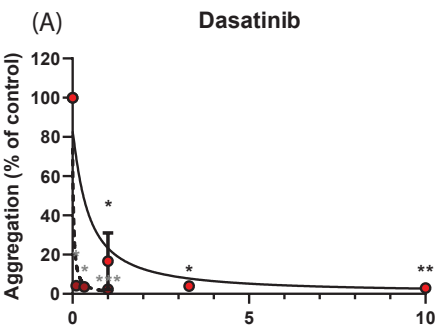

TKI concentration $(\mu \mathrm{M})$

(D)

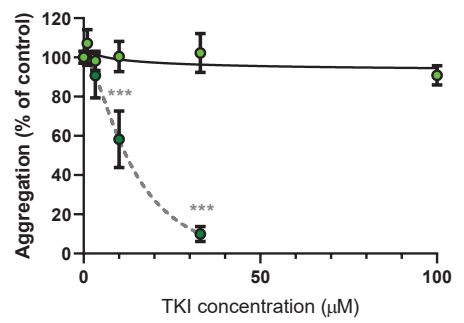

(G)

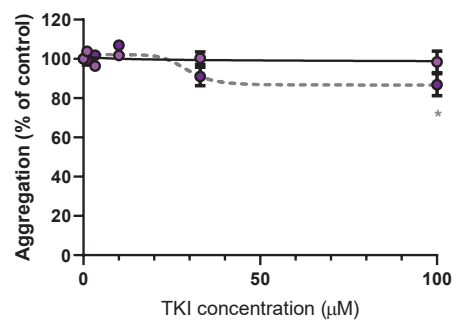

(B)

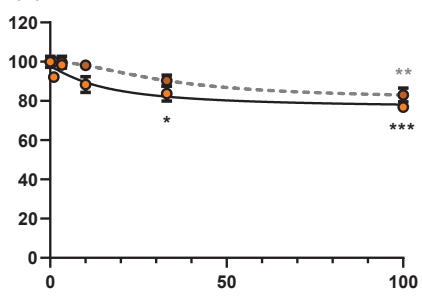

TKI concentration $(\mu \mathrm{M})$

(E)

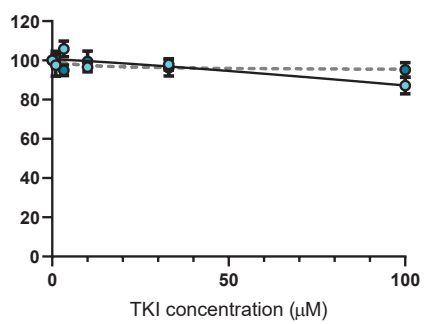

(H) Vatalanib

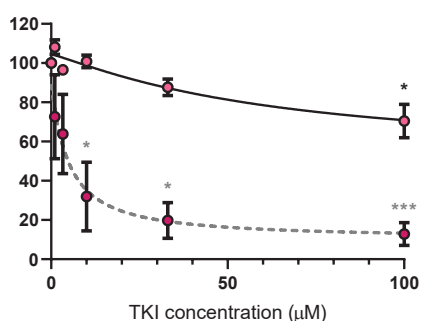

(C)

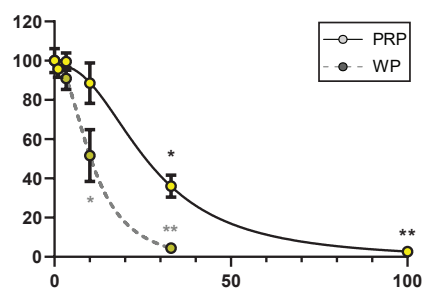

TKI concentration $(\mu \mathrm{M})$
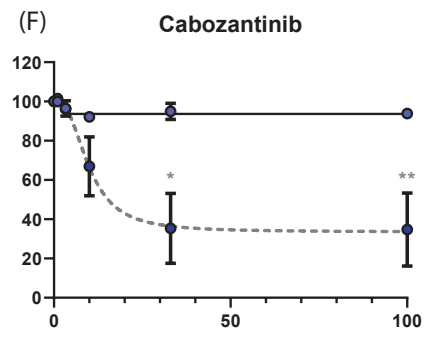

TKI concentration $(\mu \mathrm{M})$

Figure 7.2. Dose-dependent effects of TKIs on platelet aggregation induced by collagen. Washed platelets or PRP $\left(250 \times 10^{9} / \mathrm{L}\right)$ were incubated with vehicle $(<0.1 \% \mathrm{DMSO})$ or TKI for 10 minutes. Dose response curves of inhibition of aggregation induced by collagen type I (1 $\mathrm{g} / \mathrm{mL})$ for (A) dasatinib, (B) fostamatinib, (C) sunitinib, (D) pazopanib, (E) axitinib, (F) cabozantinib, (G) lapatinib or (H) vatalanib, measured in either PRP (solid line) or washed platelets (dotted line). Maximum aggregation responses were determined at 8 minutes; data are shown as means \pm $\operatorname{SEM}(n=6) .{ }^{*} p<0.05 .{ }^{* *} p<0.01,{ }^{* * *} p<0.001$. 
was used for further analysis in whole blood experiments. In contrast to washed platelets, the aggregation responses in the presence of pazopanib, cabozantinib or vatalanib were not, or barely inhibited in PRP (Figure 7.2D, F, H). In agreement with the responses in washed platelets, axitinib and lapatinib did not show altered collagen-induced aggregation in PRP (Figure 7.2E, F). For the latter set of compounds, no more than $20 \%$ inhibition was observed in PRP, for which reason the highest concentration of $33 \mu \mathrm{M}$ was used for plasma-containing experiments (Table 7.2).

\section{Whole blood thrombus formation over (non)collagen surfaces $u$ nder flow is most strongly affected by high affinity TKIs}

To explore the effects of the TKIs under physiologically relevant circumstances, we investigated platelet activation and thrombus formation under flow on coated surfaces, triggering a range of platelet receptors, i.e., GPVI, GPIb, CLEC-2, and integrins $\alpha_{6} \beta_{1}$ and $\alpha_{\| 1 \mathrm{~b}} \beta_{3}$. Blood samples were pre-incubated with one of the TKIs (applied at mostly $33 \mu \mathrm{M}$, see Table 7.2), and perfused over multiple microspots, containing collagen type I or III, or VWF co-coated with either laminin, rhodocytin, ristocetin or fibrinogen. Microscopic analysis of brightfield and fluorescence images resulted in five different thrombus parameters (P1-5) and three platelet activation markers (P6-8) (Suppl. Figure 7.1). In agreement with previous results ${ }^{34}$, different types of platelet thrombi were formed on the various surfaces with the control condition (Figure 7.3A). A systematic analysis was performed for evaluating the effects of each TKI by generating a subtraction heatmap for the eight different parameters, which were scaled (0-10) per parameter and per surface (Figure 7.3B). In contrast to the limited effect on collagen-induced aggregation, fostamatinib at $33 \mu \mathrm{M}$ was most potent in suppressing thrombus formation and platelet activation under flow, on collagen as well as non-collagen surfaces (Figure 7.3A, B). Dasatinib at $0.1 \mu \mathrm{M}$ also reduced collagen-induced thrombus formation under flow. On the VWF co-coated surfaces, this concentration of dasatinib did not affect the thrombus parameters (Figure 7.3B). Sunitinib in particular affected multiple parameters of thrombus formation on the two collagen surfaces, but showed limited effects on other surfaces. Pazopanib, axitinib and vatalanib showed weak inhibition, only affecting 1-2

Table 7.2. Concentrations of TKIs used in platelet function assays

\begin{tabular}{lccc}
\hline & \multicolumn{2}{c}{ Concentration $(\mu \mathrm{M})$} \\
\cline { 2 - 3 } TKI & WB or PRP & WP \\
\cline { 3 - 4 } \cline { 3 - 3 } Dasatinib & 0.1 & 0.1 \\
\hline Fostamatinib & 33 & 33 \\
\hline Sunitinib & 33 & 10 \\
\hline Pazopanib & 33 & 10 \\
\hline Axitinib & 75 & 33 \\
\hline Cabozantinib & 33 & 10 or 33 \\
\hline Lapatinib & 33 & 33 \\
\hline Vatalanib & 33 & 10 or 33 \\
\hline Abreviations:
\end{tabular}

Abbreviations: PRP: platelet-rich plasma; WB: whole blood; WP: washed platelets 

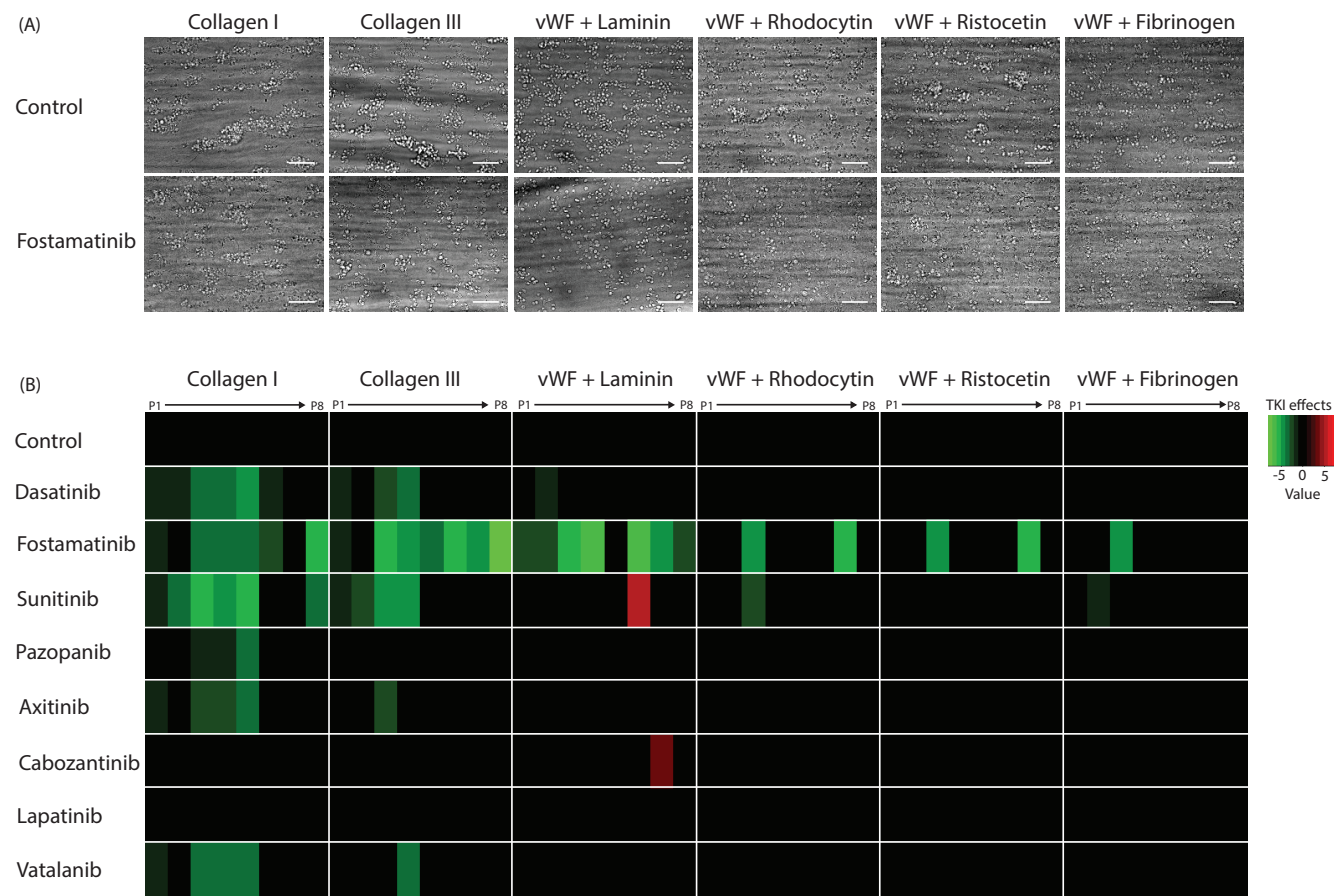

Figure 7.3. Effects of eight TKIs on whole blood thrombus formation under flow on different thrombogenic surfaces. Blood samples were pre-incubated with vehicle or TKI for 10 minutes. Samples were then recalcified in the presence of PPACK and perfused for 3.5 minutes at a wall shear rate of $1000 \mathrm{~s}^{-1}$ over six different coated surfaces. After perfusion, the formed thrombi were stained with AF568-annexin A5, FITC- $\alpha$-fibrinogen and AF647- $\alpha$-CD62P to detect PS exposure, integrin $\alpha_{\| 1} \beta_{3}$ activation and P-selectin expression, respectively. (A) Representative brightfield images of control or fostamatinib-treated blood after flow over collagen type I, collagen type III, VWF plus laminin, VWF plus rhodocytin, VWF plus ristocetin or vWF plus fibrinogen. (B) Subtraction heatmap representing significantly altered effects of parameters P1-P8 (normalized and analysed as in Suppl. Figure 7.1). P1 = morphological score of platelet adhesion and thrombus formation, $\mathrm{P} 2$ = surface area coverage of adhered platelets, $\mathrm{P} 3=$ platelet aggregate contraction score, $\mathrm{P} 4=$ platelet aggregate multilayer score, P5 = coverage of multi-layered platelet aggregation, P6 = allb $\beta 3$ activation, P7 = P-selectin expression, and P8 $=$ PS exposure. Results from vehicle control runs were set at 0 . Effects were filtered for significant alterations $(p<0.05)$ with TKIs.
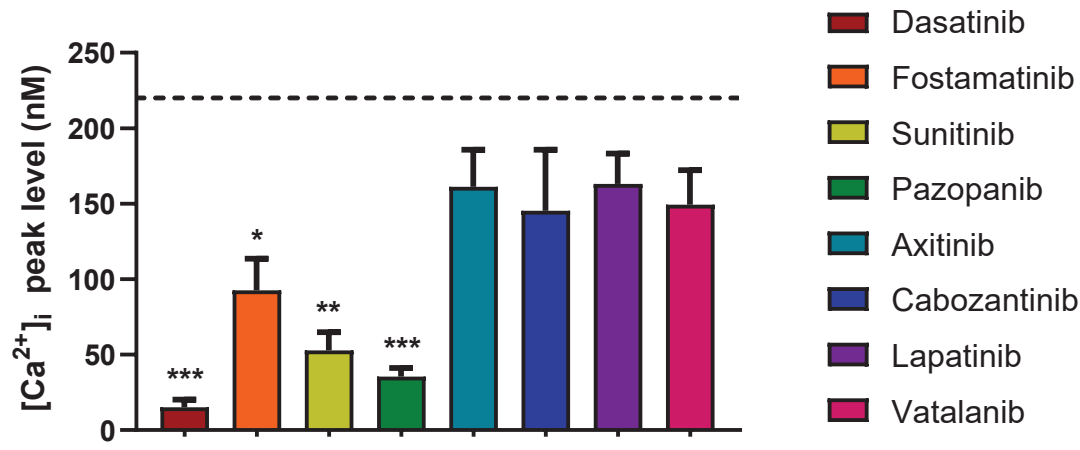

Figure 7.4. Effects of different TKIs on GPVI-induced intracellular calcium responses. Fura-2-loaded platelets $\left(200 \times 10^{9} / \mathrm{L}\right)$ were pre-incubated with vehicle or TKI for 10 minutes and stimulated with CRP-XL $(10 \mu \mathrm{g} / \mathrm{mL})$ in presence of $2 \mathrm{mM} \mathrm{CaCl}_{2}$. Histograms show maximal rises in $\left[\mathrm{Ca}^{2+}\right]_{\mathrm{i}}(\mathrm{n}=6)$. Black dotted lines indicate average of control values. Data are shown as means + SEM, $* p<0.05, * * * p<0.001$. 
parameters on collagen type I and III. Mainly the size, height and density of the thrombi were reduced (Figure 7.3B, Suppl. Figure 7.1). The inhibition of thrombus formation with dasatinib or sunitinib was in line with the effects seen in aggregation of PRP. Also, the weaker effects of the other TKIs (pazopanib, axitinib, cabozantinib, lapatinib and vatalanib) (Figure 7.3B) were in agreement with the PRP aggregation results. Together, this indicated that the TKIs with highest affinities for kinase targets in platelets (dasatinib, fostamatinib and sunitinib) had the largest impact on whole blood thrombus formation under flow.

\section{Differential effects of TKIs on GPVI-induced calcium signalling}

To further explore the inhibitory effects of the TKIs on platelet signalling, we measured the GPVI-induced intracellular calcium responses. Platelets were loaded with the probe Fura-2, preincubated with vehicle or TKI, and then stimulated with the GPVI agonist CRP-XL. Markedly, dasatinib, fostamatinib, sunitinib and pazopanib significantly decreased or even abolished the maximum intracellular calcium signal (Figure 7.4). In contrast, intracellular calcium rises were unchanged in the presence of axitinib, cabozantinib, lapatinib and vatalanib (Figure 7.4). Similar effects were seen when comparing the slope and area-under-the curve of these responses (not shown). In general, these results are in line with the observed effects on collagen-induced aggregation. However, cabozantinib and vatalanib showed 30-70\% inhibition of platelet aggregation platelets. This suggested that for dasatinib, fostamatinib, sunitinib and pazopanib the effects were mediated by early GPVI-induced signalling events in accordance with Syk or SFK inhibition, whereas for cabozantinib and vatalanib the anti-aggregatory effects are downstream of the calcium rises.

\section{Diverse effects of fostamatinib, cabozantinib and vatalanib on platelet activation markers and platelet populations}

In order to further explain the selective anti-aggregatory effects of fostamatinib, cabozantinib and vatalanib, we investigated their effects on GPVI-induced integrin $\alpha_{\| b} \beta_{3}$ activation, secretion of $\alpha$ - and $\delta$-granules and lysosomes. In general, all platelet activation markers were reduced in the presence of cabozantinib $(33 \mu \mathrm{M})$, vatalanib $(33 \mu \mathrm{M})$ and fostamatinib $(33 \mu \mathrm{M})$ (Figure 7.5A). Fostamatinib consistently inhibited all GPVI-induced activation responses. In contrast, PS exposure was not inhibited by fostamatinib in isolated platelets (Figure 7.5A). The effects of cabozantinib and vatalanib on CRP-induced platelet activation were more restricted. Cabozantinib and vatalanib both inhibited integrin $\alpha_{111} \beta_{3}$ activation, in line with the inhibition of collagen-induced aggregation in washed platelets. Furthermore, cabozantinib inhibited $\delta$-granule and lysosome secretion, whereas vatalanib resulted in reduction of $\alpha$-granule secretion. PS exposure was also not affected by cabozantinib and vatalanib. As sustained high calcium rises are a prerequisite for PS exposure, the absence of effects on PS exposure by the TKIs is in agreement 
with the calcium responses that remained unchanged by cabozantinib and vatalanib. Hence, cabozantinib and vatalanib suppress platelet integrin activation and secretion at doses that do not influence the calcium response.

To evaluate a differential sensitivity of platelets towards these TKIs in terms of activation markers, we determined how the formation of activated platelet populations was influenced
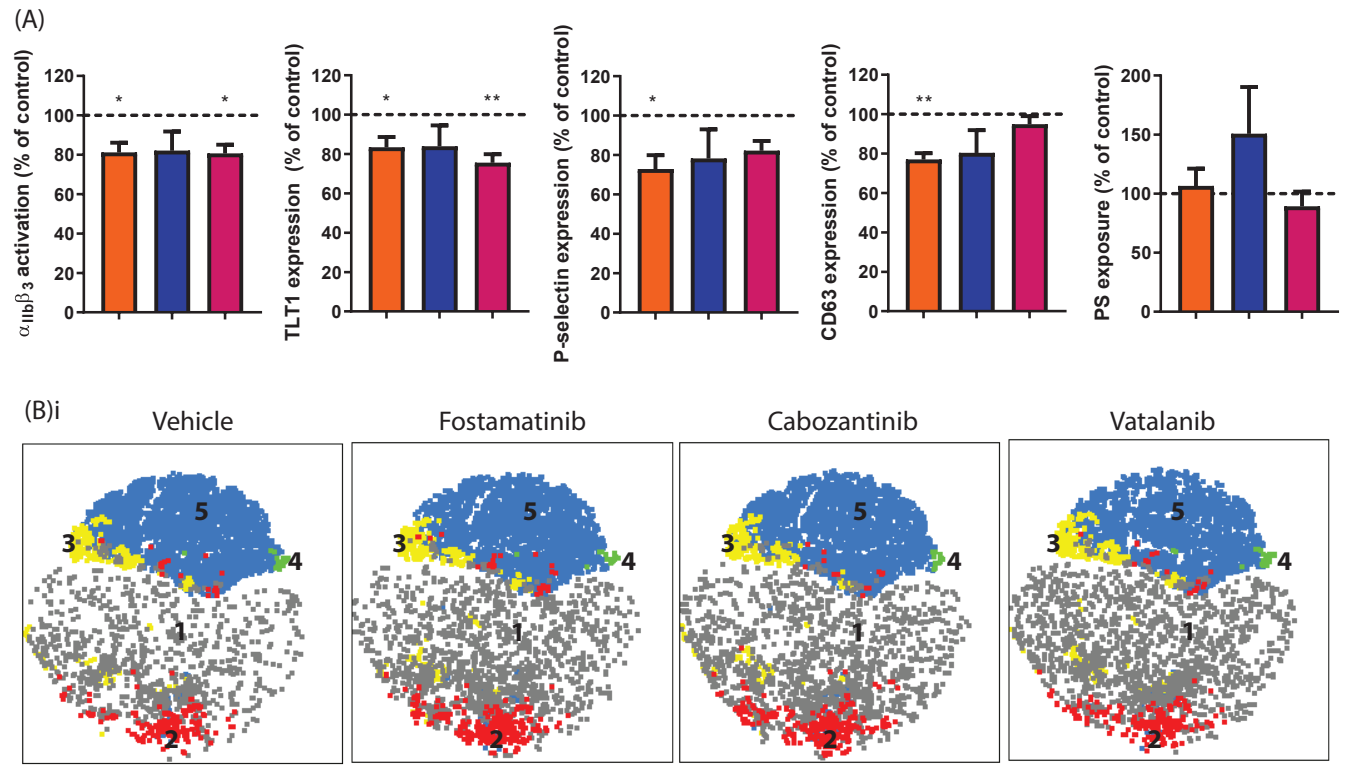

(B)ii
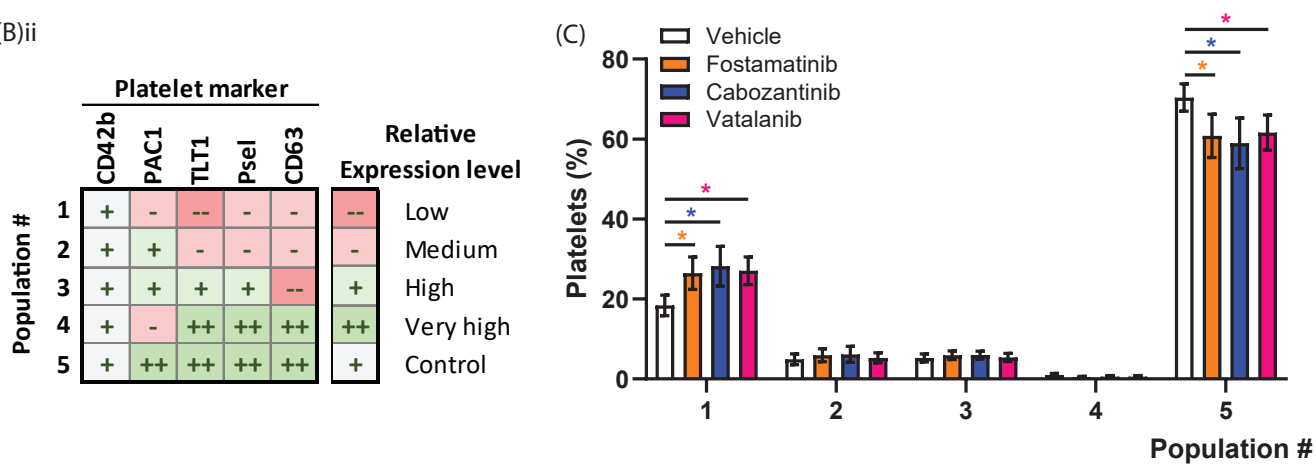

Figure 7.5. Effect of TKIs on populations of activated platelets in response of GPVI. Washed platelets $\left(25 \times 10^{9} / \mathrm{L}\right)$ were incubated with vehicle, fostamatinib $(33 \mu \mathrm{M})$, cabozantinib $(33 \mu \mathrm{M})$ or vatalanib $(33 \mu \mathrm{M})$ for 10 minutes. Platelet activation was induced by $5 \mu \mathrm{g} / \mathrm{mL}$ CRP-XL for 15 minutes and simultaneously labelled for activated integrin $\alpha_{\| 1} \beta_{3}$ (PAC1-FITC), $\alpha$-granule secretion ( $\alpha$ TLT1-BV421, $\alpha$ CD62P-PerCP Cy5.5) and $\delta$-granule/lysosome secretion ( $\alpha$ CD63-APC). Separately, PS exposure was induced using $5 \mathrm{\mu g} / \mathrm{mL}$ CRP-XL plus $4 \mathrm{nM}$ thrombin for 60 minutes at $37^{\circ} \mathrm{C}$, labelling was with annexin $\mathrm{A} 5-\mathrm{PE}$. (A) Histograms of integrin $\alpha_{\| b} \beta_{3}$ activation, TLT1, P-selectin and CD63 expression as well as PS exposure as percentages of control condition. (B) Distribution profiles of platelet populations after stimulation with CRP-XL. (i) 2D plots visualising the platelet fractions per population in the presence of vehicle, fostamatinib, cabozantinib or vatalanib. (ii) Characterisation of CRP-XL-induced platelet populations based on relative expression levels of activation markers determined by FlowJo analysis, using tSNE and FlowSOM plugins. (C) Histograms visualising the platelet fractions per population in the presence of vehicle (white bars), or fostamatinib, cabozantinib or vatalanib (coloured bars). Data are shown as means $\pm S E M(n=6)$, $* p<0.05, * * p<0.01$. 
by the presence of fostamatinib, cabozantinib or vatalanib. Comparison of the four different activation markers, reflecting stages of granular secretion and integrin activation, rendered up to five different populations (Figure 7.5Bii) upon stimulation with CRP-XL. These were ranging from low up to very high expression levels for all markers with an equal expression of the control marker GPIb throughout the samples. The changes in distribution of these populations in the presence of the TKIs were illustrated by 2D scatterplots that visualised the distribution of the populations (Figure 7.5Bi). Population 1 with low-level platelet activation (resting platelets), appeared to be significantly increased with fostamatinib, cabozantinib or vatalanib upon GPVI stimulation. On the other hand, population 5 with high activation of $\alpha_{\| 1 /} \beta_{3}$ and high secretion of $\alpha$-, $\delta$-granules and lysosomes was decreased in the presence of these TKIs (Figure 7.5C), whereas no changes were observed in other specialised platelet populations. This approach demonstrated
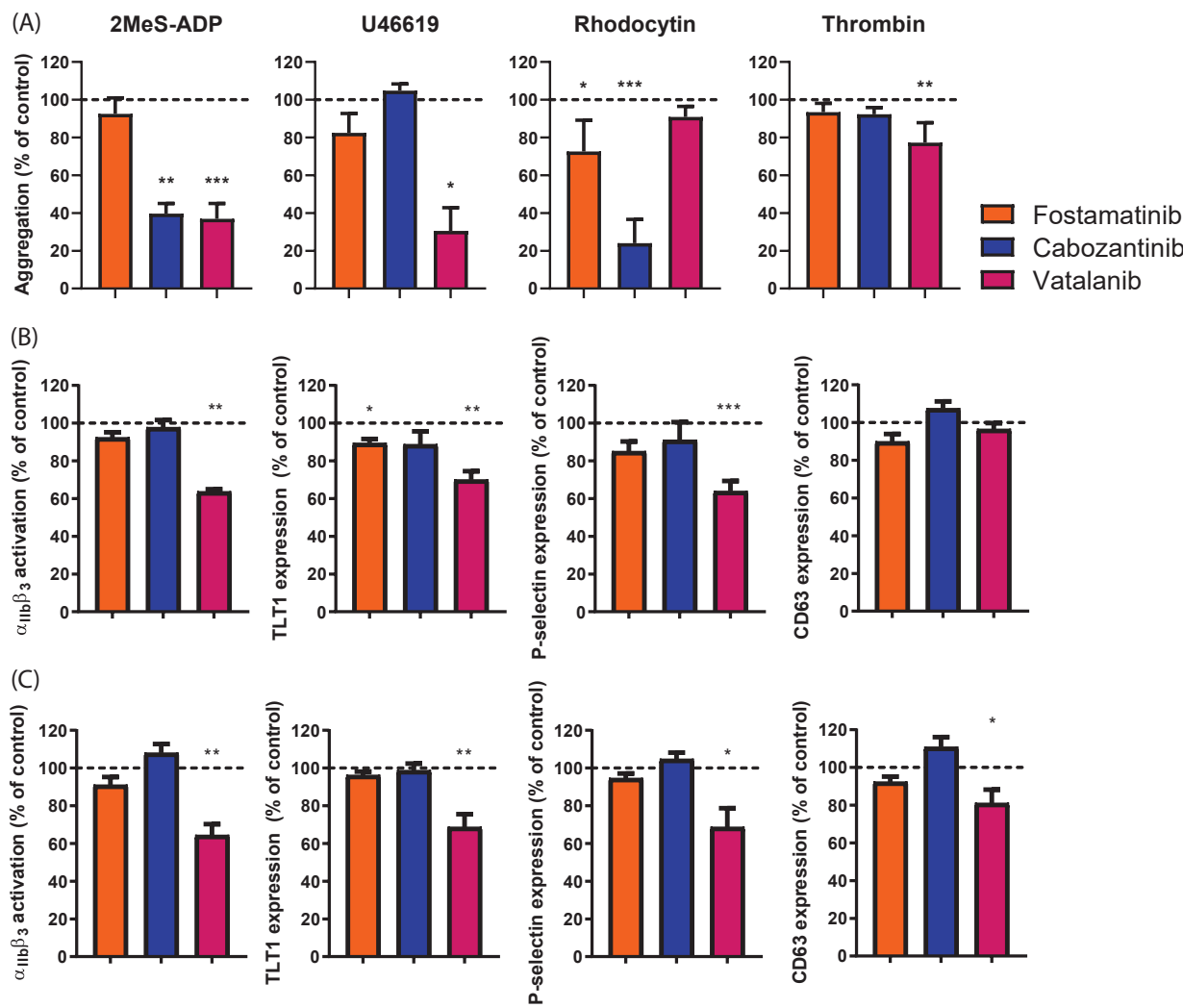

Figure 7.6. Platelet aggregation and activation induced by different activation pathways in presence of different TKIs. (A) Washed platelets $\left(250 \times 10^{9} / \mathrm{L}\right)$ were incubated with vehicle (control), fostamatinib (33 $\left.\mu \mathrm{M}\right)$, cabozantinib (33 $\mu \mathrm{M})$ or vatalanib $(33 \mu \mathrm{M})$ for 10 minutes. Platelet aggregation was then induced with $1 \mu \mathrm{M} 2 \mathrm{MeS}-\mathrm{ADP}, 1 \mu \mathrm{M}$ U46619 (thromboxane $\mathrm{A}_{2}$ analogue), $1 \mathrm{\mu g} / \mathrm{mL}$ rhodocytin or $4 \mathrm{nM}$ thrombin. Aggregation responses were measured for 8 minutes. Histograms show aggregation as percentage of control (dotted line). (B-C) Washed platelets $\left(25 \times 10^{9} / \mathrm{L}\right)$ were incubated with vehicle (control) or TKI for 10 minutes. Platelets were activated by (B) $2 \mu \mathrm{M} 2 \mathrm{MeS}-\mathrm{ADP}$, or (C) $4 \mathrm{nM}$ thrombin for 15 minutes. Simultaneous labelling was for activated integrin $\alpha_{11 b} \beta_{3}$ (PAC1-FITC), $\alpha$-granule secretion ( $\alpha$ TLT1-BV421, $\alpha$ CD62PPerCP Cy5.5) and $\delta$-granule/lysosome secretion ( $\alpha$ CD63-APC). Histograms of integrin $\alpha_{116} \beta_{3}$ activation, TLT1, P-selectin and CD63 expression, as percentages of control condition (dotted line). Data are shown as means $\pm \operatorname{SEM}(n=6), * p<0.05$. $* *$ $\mathrm{p}<0.01, * * * \mathrm{p}<0.001$. 
the simultaneous inhibition of secretory and integrin-activating platelet responses by these TKIs.

In whole blood perfusion experiments, we observed inhibitory effects of fostamatinib on thrombus formation on VWF co-coated surfaces. To determine effects of fostamatinib, cabozantinib and vatalanib on platelet activation induced by other agonists, platelet aggregation was investigated in response to ADP, the thromboxane $A_{2}$ analogue U46619, the CLEC-2 agonist rhodocytin or thrombin. Fostamatinib only reduced rhodocytin-induced aggregation (Figure 7.6A), in agreement with Syk signalling underneath CLEC $-2^{26}$. In contrast, cabozantinib significantly inhibited the aggregation responses to ADP and rhodocytin, while vatalanib prominently reduced the responses to ADP and U46619, and slightly to thrombin (Figure 7.6A). In line with these aggregation results, vatalanib suppressed ADP- and thrombin-induced integrin $\alpha_{\| 1} \beta_{3}$ activation, TLT1 expression and P-selectin expression (Figure 7.6B, C). Upon thrombin stimulation also $\delta$-granule and lysosome secretion were partially inhibited (Figure 7.6C). In contrast to the observed reduction in ADP-induced aggregation with cabozantinib, no inhibition of platelet activation was observed in response to ADP (Figure 7.6B, C). Taken together, this suggested that the latter two compounds inhibited signalling entities in pathways other than induced by GPVI.

\section{Discussion}

In this work we explored the antiplatelet properties of the TKIs dasatinib, fostamatinib, sunitinib, pazopanib, cabozantinib, axitinib, lapatinib, and vatalanib, to investigate the possible repurposing of these compounds as antiplatelet drugs. Overall, we observed that the TKIs with the highest reported affinities for targets in platelets (dasatinib, fostamatinib and sunitinib) at doses used showed the strongest inhibition on collagen-dependent whole blood thrombus formation under flow. Furthermore, fostamatinib was the most effective inhibitor of thrombus formation also on VWF plus rhodocytin, or VWF plus laminin. In the presence of other TKIs thrombus formation was no more than mildly reduced only on collagen. Intracellular calcium measurements and activation responses in isolated platelets showed that the inhibitory effects of dasatinib, fostamatinib, sunitinib and pazopanib were in line with proposed inhibitory effects on Syk and/or SFK, i.e., downstream of GPVI-induced platelet activation. At the doses tested, calcium measurements indicated that effects of the low-affinity inhibitors cabozantinib and vatalanib are downstream of phospholipase $\mathrm{C}$ p phosphorylation.

The present strong effects of the active metabolite of fostamatinib (R406) with high affinity for Syk on thrombus formation and PS exposure under flow are in line with a previous study using the selective Syk inhibitor PRT-060318 37 . However, our results obtained in isolated platelets are in contrast to other studies ${ }^{26,42}$. These studies demonstrated significant inhibition of collagen- or rhodocytin-induced platelet aggregation with low concentrations of R406, whereas we observed only minor inhibition at higher concentrations. The difference may be due to longer incubation time of the platelets with the inhibitor in our study. As fostamatinib is a reversible 


\section{Chapter 7}

inhibitor, the incubation time could be of importance, however, we did observe strong inhibition in whole blood perfusion experiments using the same pre-incubation time. Furthermore, others have incubated with fostamatinib for even longer time-periods in platelets as well as other cell types, making transient inhibition unlikely ${ }^{27,43}$. In addition, fostamatinib strongly inhibited GPVI-induced PS exposure in whole blood thrombus formation under flow, however, in isolated platelets this response was not affected. PS exposure is dependent on sustained high intracellular calcium levels ${ }^{44}$, which we showed to be inhibited by fostamatinib. As PS exposure is triggered by dual stimulation of CRP and thrombin in our setting, this might overcome the partially decreased calcium signal induced by GPVI-stimulation in the presence of fostamatinib and thereby resulting in normal PS exposure.

Several of the investigated TKIs are used for treatment of metastatic renal cell carcinoma (mRCC) patients, i.e., sunitinib, pazopanib, cabozantinib and axitinib. Our results with sunitinib and pazopanib are consistent with a moderate inhibition of SFK, Syk and other relevant tyrosine kinases in platelets (Figure 7.1). For both compounds only mild bleeding effects have been reported $^{30}$. Cabozantinib is used mainly as second- or third-line treatment for mRCC patients, and axitinib (combined with pembrolizumab) in first line mRCC, but their effects on platelets have not been investigated thus far. For cabozantinib, we report a reduced collagen-, CLEC-2- and ADP-induced aggregation in washed platelets, but no effect on thrombus formation. This is in line with no more than limited reported bleeding ${ }^{30}$. Axitinib at the dose of $33 \mu \mathrm{M}$ used was essentially ineffective in most assays. We did observe limited effects on thrombus height and contraction in whole blood perfused over collagen. Notably, axitinib is described to induce mild (and in rare cases severe) thrombocytopenia in $15-20 \%$ of the patients ${ }^{45-47}$, which points to a megakaryocytic rather than platelet function defect contributing to the reported bleeding ${ }^{48,49}$.

Lapatinib and vatalanib have been reported to have limited affinity for tyrosine kinases in platelets and remain without reported bleeding ${ }^{24}$. In agreement with Li et al. ${ }^{50}$, lapatinib did not alter thrombus formation in whole blood perfusion, as well as intracellular calcium responses and aggregation in isolated platelets. Surprisingly, in the presence of vatalanib, we did observe inhibition of thrombus size, height, and contraction on collagen under flow at $33 \mu \mathrm{M}$, which is in contrast to the observations of $\mathrm{Li}$ et al., but the used concentration was not reported ${ }^{50}$. In addition, in isolated platelets vatalanib inhibited collagen-, ADP-, TxA ${ }_{2}$ - and thrombin-induced aggregation, as well as platelet activation. As GPVI-induced intracellular calcium rises were not affected by vatalanib, this suggested that vatalanib affects platelet signalling downstream of calcium. Vatalanib was developed to inhibit all VEGF receptors, and is mostly used for treatment of patients with colorectal cancer ${ }^{51}$. As no platelet tyrosine kinases are reported to be affected by this compound, further research is required to investigate the inhibitory mechanism in platelets.

In washed platelets, dasatinib, sunitinib, pazopanib, cabozantinib, and vatalanib prominently inhibited collagen-induced aggregation, while in PRP this response was unaffected 
(cabozantinib and pazopanib) or required a higher concentration of the TKI to achieve inhibition (dasatinib, sunitinib and vatalanib). For pazopanib, this is in line with previous findings $s^{28}$, and explained by high plasma binding ${ }^{52-55}$. Indeed, in patients with TKI treatment, the bioavailability of the drug is considered to be influenced by plasma binding with implications for drug toxicity and response ${ }^{56}$. In line with this, for the TKIs that did not or limitedly inhibited collagen-induced aggregation in PRP, we also observed no or only limited effects on thrombus formation under flow.

For the doses used of fostamatinib, cabozantinib and vatalanib we investigated effects on secretion and integrin activation in more detail. Most consistent effects were observed with fostamatinib, which moderately inhibited all GPVI-induced activation responses. Cabozantinib and vatalanib inhibited integrin $\alpha_{\| b} \beta_{3}$ activation along with aggregation. Interestingly, cabozantinib mildly suppressed $\delta$-granule and lysosome secretion, whereas vatalanib reduced the $\alpha$-granule secretion. The reason for this difference is unclear. Furthermore, we used two markers to measure of $\alpha$-granule secretion, namely P-selectin (CD62P) and TLT-1. Although, in general we observed similar results using both markers with several agonists as well as inhibitors, it has been reported that TLT1 is more sensitive marker for platelet activation than P-selectin ${ }^{57}$. When a soluble TLT-1 is released in the plasma, it enhances platelet aggregation ${ }^{58,59}$ and has been demonstrated to regulate early clot formation though the stabilization of $\alpha_{11 \mathrm{~b}} \beta_{3}$ outside-in signalling ${ }^{60}$. Hence, this may provide an additional mechanism of the inhibitory effect of vatalanib.

We determined the distribution of the single platelet activation markers among different populations to further zoom in on the effects of fostamatinib, cabozantinib or vatalanib on GPVI-induced platelet activation. In general, in the presence of TKIs the population with highly expressed activation markers was decreased, whereas the population with negative expression of these markers was increased. This integrated approach suggested that pre-incubation with TKIs may affect the general responsiveness of the heterogeneous platelet populations ${ }^{61}$.

In conclusion, we observed that fostamatinib was the most effective inhibitor of thrombus formation on collagen under flow, and that pazopanib, sunitinib, dasatinib, axitinib, and vatalanib also mildly reduced this process. Furthermore, the TKIs showed variable inhibiting effects in isolated platelets stimulated with several agonists. As especially fostamatinib, sunitinib, pazopanib, and vatalanib have been associated with mostly manageable or no bleeding events, these may be promising candidates to explore further as antiplatelet drugs. Clinicians should be aware of the antiplatelet properties of TKIs in general, especially when patients are already treated with antiplatelet or anticoagulant drugs.

\section{Acknowledgments}

JAE was financially supported by the Interdisciplinary Centre for Clinical Research (IZKF) of Münster University (grant: Ebl/009/21). 


\section{Author contributions}

Conceptualization, MJEK, JWMH and PEJM; formal analysis, BMET, AV and DIF; investigation, BMET, AV and DIF; resources, JAE and MJBA; writing — original draft preparation, BMET and MJEK; writing-review and editing, BMET and MJEK; visualization BMET; supervision, MJEK, JWMH and PEJM; project administration, MJEK; funding acquisition, MJEK, JWMH and PEJM. All authors have read and agreed to the published version of the manuscript.

\section{References}

1. WHO. The top 10 causes of death. WHO (World Health Organization), 2020.

2. Bentzon JF, Otsuka F, Virmani R, Falk E. Mechanisms of plaque formation and rupture. Circ Res. 2014; 114: 1852-66.

3. Libby P, Pasterkamp G, Crea F, Jang IK. Reassessing the mechanisms of acute coronary syndromes. Circ Res. 2019; 124: 150-60.

4. Roffi M, Patrono C, Collet JP, et al. 2015 ESC Guidelines for the management of acute coronary syndromes in patients presenting without persistent ST-segment elevation: Task force for the management of acute coronary syndromes in patients presenting without persistent ST-segment elevation of the European society of cardiology (ESC). Eur Heart J. 2016; 37: 267-315.

5. McFadyen JD, Schaff M, Peter K. Current and future antiplatelet therapies: emphasis on preserving haemostasis. Nat Rev Cardiol. 2018; 15: 181-91.

6. Harbi MH, Smith CW, Nicolson PLR, Watson SP, Thomas MR. Novel antiplatelet strategies targeting GPVI, CLEC-2 and tyrosine kinases. Platelets. 2021; 32: 29-41.

7. Nieswandt B, Brakebusch C, Bergmeier W, et al. Glycoprotein VI but not alpha2beta1 integrin is essential for platelet interaction with collagen. Embo j. 2001; 20: 2120-30.

8. Alshehri OM, Hughes CE, Montague S, et al. Fibrin activates GPVI in human and mouse platelets. Blood. 2015; 126: 1601-8.

9. Mangin $\mathrm{PH}$, Onselaer $\mathrm{MB}$, Receveur $\mathrm{N}$, et al. Immobilized fibrinogen activates human platelets through glycoprotein VI. Haematologica. 2018; 103: 898-907.

10. Nieswandt B, Schulte V, Bergmeier W, et al. Long-term antithrombotic protection by in vivo depletion of platelet glycoprotein VI in mice. J Exp Med. 2001; 193: 459-69.

11. Lockyer S, Okuyama K, Begum S, et al. GPVI-deficient mice lack collagen responses and are protected against experimentally induced pulmonary thromboembolism. Thromb Res. 2006; 118: 371-80.

12. Schönberger T, Siegel-Axel D, BussI R, et al. The immunoadhesin glycoprotein VI-Fc regulates arterial remodelling after mechanical injury in ApoE-/- mice. Cardiovasc Res. 2008; 80: 131-7.

13. Kuijpers MJE, Gilio K, Reitsma S, et al. Complementary roles of platelets and coagulation in thrombus formation on plaques acutely ruptured by targeted ultrasound treatment: a novel intravital model. J Thromb Haemost. 2009; 7: 152-61.

14. Moroi M, Jung SM, Okuma M, Shinmyozu K. A patient with platelets deficient in glycoprotein VI that lack both collagen-induced aggregation and adhesion. J Clin Invest. 1989; 84: 1440-5.

15. Nagy M, Perrella G, Dalby A, et al. Flow studies on human GPVI-deficient blood under coagulating and noncoagulating conditions. Blood Adv. 2020; 4: 2953-61.

16. Suzuki-Inoue $\mathrm{K}$, Kato $\mathrm{Y}$, Inoue $\mathrm{O}$, et al. Involvement of the snake toxin receptor CLEC-2, in podoplaninmediated platelet activation, by cancer cells. J Biol Chem. 2007; 282: 25993-6001.

17. Suzuki-Inoue K, Inoue O, Ding G, et al. Essential in vivo roles of the C-type lectin receptor CLEC2: embryonic/neonatal lethality of CLEC-2-deficient mice by blood/lymphatic misconnections and impaired thrombus formation of CLEC-2-deficient platelets. J Biol Chem. 2010; 285: 24494-507.

18. May F, Hagedorn I, Pleines I, et al. CLEC-2 is an essential platelet-activating receptor in hemostasis and thrombosis. Blood. 2009; 114: 3464-72.

19. Bender M, May F, Lorenz V, et al. Combined in vivo depletion of glycoprotein VI and C-type lectin-like receptor 2 severely compromises hemostasis and abrogates arterial thrombosis in mice. Arterioscler Thromb Vasc Biol. 2013; 33: 926-34. 
20. Gitz E, Pollitt AY, Gitz-Francois JJ, et al. CLEC-2 expression is maintained on activated platelets and on platelet microparticles. Blood. 2014; 124: 2262-70.

21. Versteeg HH, Heemskerk JWM, Levi M, Reitsma PH. New fundamentals in hemostasis. Physiol Rev. 2013; 93: 327-58.

22. Rivera J, Lozano ML, Navarro-Núñez L, Vicente V. Platelet receptors and signaling in the dynamics of thrombus formation. Haematologica. 2009; 94: 700-11.

23. Tsiamis AC, Hayes P, Box H, Goodall AH, Bell PR, Brindle NP. Characterization and regulation of the receptor tyrosine kinase Tie-1 in platelets. J Vasc Res. 2000; 37: 437-42.

24. Tullemans BME, Heemskerk JWM, Kuijpers MJE. Acquired platelet antagonism: off-target antiplatelet effects of malignancy treatment with tyrosine kinase inhibitors. J Thromb Haemost. 2018; 16: 168699.

25. Levade M, Severin S, Gratacap MP, Ysebaert L, Payrastre B. Targeting kinases in cancer therapies: adverse effects on blood platelets. Curr Pharm Des. 2016; 22: 2315-22.

26. Spalton JC, Mori J, Pollitt AY, Hughes CE, Eble JA, Watson SP. The novel Syk inhibitor R406 reveals mechanistic differences in the initiation of GPVI and CLEC-2 signaling in platelets. J Thromb Haemost. 2009; 7: 1192-9.

27. Braselmann S, Taylor V, Zhao H, et al. R406, an orally available spleen tyrosine kinase inhibitor blocks fc receptor signaling and reduces immune complex-mediated inflammation. J Pharmacol Exp Ther. 2006; 319: 998-1008.

28. Tullemans BME, Nagy M, Sabrkhany S, et al. Tyrosine kinase inhibitor pazopanib inhibits platelet procoagulant activity in renal cell carcinoma patients. Front Cardiovasc Med. 2018; 5: 142.

29. Sabrkhany S, Griffioen AW, Pineda S, et al. Sunitinib uptake inhibits platelet function in cancer patients. Eur J Cancer. 2016; 66: 47-54.

30. Crist M, Hansen E, Chablani L, Guancial E. Examining the bleeding incidences associated with targeted therapies used in metastatic renal cell carcinoma. Crit Rev Oncol Hematol. 2017; 120: 151-62.

31. Bergmeier W, Bouvard D, Eble JA, et al. Rhodocytin (aggretin) activates platelets lacking alpha 2 beta 1 integrin, glycoprotein VI, and the ligand-binding domain of glycoprotein Ibalpha. J Biol Chem. 2001; 276: 25121-6.

32. van der Meijden PEJ, Feijge MA, Giesen PL, Huijberts M, van Raak LP, Heemskerk JWM. Platelet P2Y12 receptors enhance signalling towards procoagulant activity and thrombin generation. A study with healthy subjects and patients at thrombotic risk. Thromb Haemost. 2005; 93: 1128-36.

33. Mattheij NJ, Gilio K, van Kruchten R, et al. Dual mechanism of integrin alphallbbeta3 closure in procoagulant platelets. J Biol Chem. 2013; 288: 13325-36.

34. de Witt SM, Swieringa F, Cavill R, et al. Identification of platelet function defects by multi-parameter assessment of thrombus formation. Nat Commun. 2014; 5: 4257.

35. van Geffen JP, Brouns SLN, Batista J, et al. High-throughput elucidation of thrombus formation reveals sources of platelet function variability. Haematologica. 2019; 104: 1256-67.

36. Feijge MA, van Pampus EC, Lacabaratz-Porret C, et al. Inter-individual variability in $\mathrm{Ca} 2+$ signalling in platelets from healthy volunteers: effects of aspirin and relationship with expression of endomembrane Ca2+-ATPases. Br J Haematol. 1998; 102: 850-9.

37. Jooss NJ, De Simone I, Provenzale I, et al. Role of platelet glycoprotein VI and tyrosine kinase Syk in thrombus formation on collagen-like surfaces. Int J Mol Sci. 2019; 20.

38. Monaco G, Chen H, Poidinger M, Chen J, de Magalhães JP, Larbi A. FlowAl: automatic and interactive anomaly discerning tools for flow cytometry data. Bioinformatics. 2016; 32: 2473-80.

39. Van Gassen S, Callebaut B, Van Helden MJ, et al. FlowSOM: Using self-organizing maps for visualization and interpretation of cytometry data. Cytometry A. 2015; 87: 636-45.

40. Gratacap MP, Martin V, Valéra MC, et al. The new tyrosine-kinase inhibitor and anticancer drug dasatinib reversibly affects platelet activation in vitro and in vivo. Blood. 2009; 114: 1884-92.

41. EMA. Sprycel, INN-dasatinib Scientific Discussion. 2006.

42. Lhermusier T, van Rottem J, Garcia C, et al. The Syk-kinase inhibitor R406 impairs platelet activation and monocyte tissue factor expression triggered by heparin-PF4 complex directed antibodies. J Thromb Haemost. 2011; 9: 2067-76.

43. Simpson SR, Singh MV, Dewhurst S, Schifitto G, Maggirwar SB. Platelets function as an acute viral reservoir during HIV-1 infection by harboring virus and T-cell complex formation. Blood Adv. 2020; 


\section{4: 4512-21.}

44. Heemskerk JWM, Mattheij NJA, Cosemans JMEM. Platelet-based coagulation: different populations, different functions. J Thromb Haemost. 2013; 11: 2-16.

45. Rini BI. Targeted therapy for patients with renal-cell carcinoma. Lancet Oncol. 2011; 12: 1085-7.

46. Rini BI. Metastatic renal cell carcinoma: many treatment options, one patient. J Clin Oncol. 2009; 27: 3225-34.

47. Koksal UI, Goffin J, Lewis B, et al. A case report with severe thrombocytopenia induced by axitinib. Case Rep Hematol. 2020; 2020: 7520783.

48. Gunnarsson O, Pfanzelter NR, Cohen RB, Keefe SM. Evaluating the safety and efficacy of axitinib in the treatment of advanced renal cell carcinoma. Cancer Manag Res. 2015; 7: 65-73.

49. Abdul Sater H, Ghandi AS, Dainer P, Pantin J. Receptor tyrosine kinases in human platelets: a review of expression, function and inhibition in relation to the risk of bleeding or thrombocytopenia from phase I through phase III trials. Journal of Cancer Prevention \& Current Research. 2017; 8.

50. Li R, Grosser T, Diamond SL. Microfluidic whole blood testing of platelet response to pharmacological agents. Platelets. 2017; 28: 457-62.

51. Scott EN, Meinhardt G, Jacques C, Laurent D, Thomas AL. Vatalanib: the clinical development of a tyrosine kinase inhibitor of angiogenesis in solid tumours. Expert Opin Investig Drugs. 2007; 16: 367-79.

52. O'Brien Z, Moghaddam MF. A systematic analysis of physicochemical and ADME properties of all small molecule kinase inhibitors approved by US FDA from january 2001 to october 2015. Curr Med Chem. 2017; 24: 3159-84.

53. Podoll T, Pearson PG, Evarts J, et al. Bioavailability, biotransformation, and excretion of the covalent Bruton tyrosine kinase inhibitor acalabrutinib in rats, dogs, and humans. Drug Metab Dispos. 2019; 47: 145-54.

54. McAdoo SP, Tam FW. Fostamatinib Disodium. Drugs Future. 2011; 36: 273.

55. Wang X, Owzar K, Gupta $P$, et al. Vatalanib population pharmacokinetics in patients with myelodysplastic syndrome: CALGB 10105 (Alliance). Br J Clin Pharmacol. 2014; 78: 1005-13.

56. Li J, Brahmer J, Messersmith W, Hidalgo M, Baker SD. Binding of gefitinib, an inhibitor of epidermal growth factor receptor-tyrosine kinase, to plasma proteins and blood cells: in vitro and in cancer patients. Invest New Drugs. 2006; 24: 291-7.

57. Smith CW, Raslan Z, Parfitt L, et al. TREM-like transcript 1: a more sensitive marker of platelet activation than P-selectin in humans and mice. Blood Adv. 2018; 2: 2072-8.

58. Washington AV, Schubert RL, Quigley L, et al. A TREM family member, TLT-1, is found exclusively in the alpha-granules of megakaryocytes and platelets. Blood. 2004; 104: 1042-7.

59. Washington AV, Gibot S, Acevedo I, et al. TREM-like transcript-1 protects against inflammationassociated hemorrhage by facilitating platelet aggregation in mice and humans. J Clin Invest. 2009; 119: 1489-501.

60. Morales-Ortiz J, Reyes FS, Santiago O, et al. TLT-1- controls early thrombus formation and stability by facilitating allbß3 outside-in signaling in mice. Int J Adv Res (Indore). 2018; 6: 1143-9.

61. Baaten CCFMJ, Ten Cate H, van der Meijden PEJ, Heemskerk JWM. Platelet populations and priming in hematological diseases. Blood Rev. 2017; 31: 389-99.

62. Fabian MA, Biggs WH, 3rd, Treiber DK, et al. A small molecule-kinase interaction map for clinical kinase inhibitors. Nat Biotechnol. 2005; 23: 329-36.

63. Karaman MW, Herrgard S, Treiber DK, et al. A quantitative analysis of kinase inhibitor selectivity. Nat Biotechnol. 2008; 26: 127-32.

64. Davis $\mathrm{MI}$, Hunt JP, Herrgard $\mathrm{S}$, et al. Comprehensive analysis of kinase inhibitor selectivity. Nat Biotechnol. 2011; 29: 1046-51.

65. Yakes FM, Chen J, Tan J, et al. Cabozantinib (XL184), a novel MET and VEGFR2 inhibitor, simultaneously suppresses metastasis, angiogenesis, and tumor growth. Mol Cancer Ther. 2011; 10: 2298-308.

66. Yu H, Steeghs N, Nijenhuis CM, Schellens JH, Beijnen JH, Huitema AD. Practical guidelines for therapeutic drug monitoring of anticancer tyrosine kinase inhibitors: focus on the pharmacokinetic targets. Clin Pharmacokinet. 2014; 53: 305-25.

67. Aguilera DG, Tsimberidou AM. Dasatinib in chronic myeloid leukemia: a review. Ther Clin Risk Manag. 2009; 5: 281-9. 
68. Je Y, Schutz FA, Choueiri TK. Risk of bleeding with vascular endothelial growth factor receptor tyrosine-kinase inhibitors sunitinib and sorafenib: a systematic review and meta-analysis of clinical trials. Lancet Oncol. 2009; 10: 967-74.

69. Sabrkhany S, Griffioen AW, Pineda S, et al. Sunitinib uptake inhibits platelet function in cancer patients. Eur J Cancer. 2016; 66: 47-54.

70. Choueiri TK, Escudier B, Powles T, et al. Cabozantinib versus everolimus in advanced renal-cell carcinoma. N Engl J Med. 2015; 373: 1814-23.

71. Blackwell KL, Burstein HJ, Storniolo AM, et al. Randomized study of lapatinib alone or in combination with trastuzumab in women with ErbB2-positive, trastuzumab-refractory metastatic breast cancer. J Clin Oncol. 2010; 28: 1124-30.

72. Hecht JR, Trarbach T, Hainsworth JD, et al. Randomized, placebo-controlled, phase III study of firstline oxaliplatin-based chemotherapy plus PTK787/ZK 222584, an oral vascular endothelial growth factor receptor inhibitor, in patients with metastatic colorectal adenocarcinoma. J Clin Oncol. 2011; 29: 1997-2003. 


\section{Supplemental data}
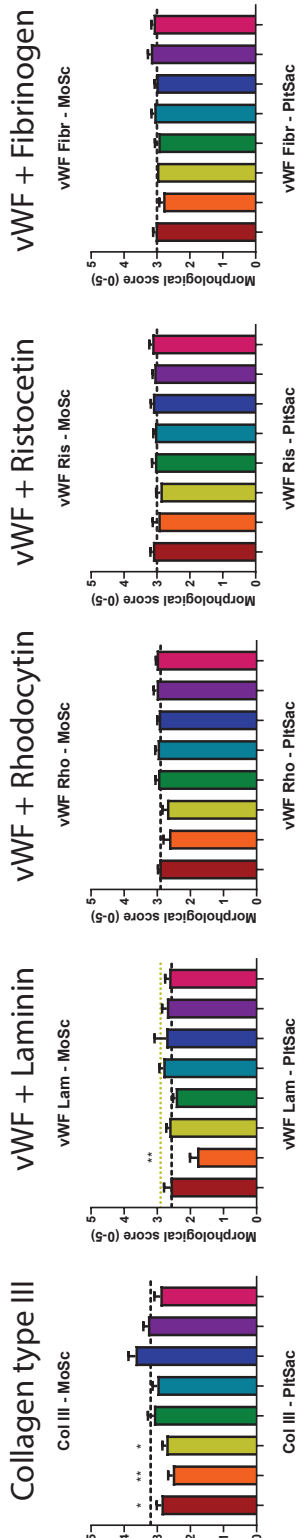

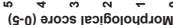

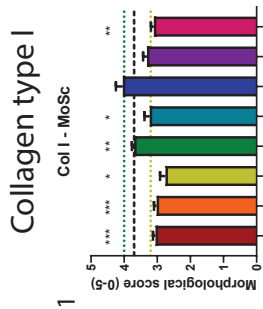

도
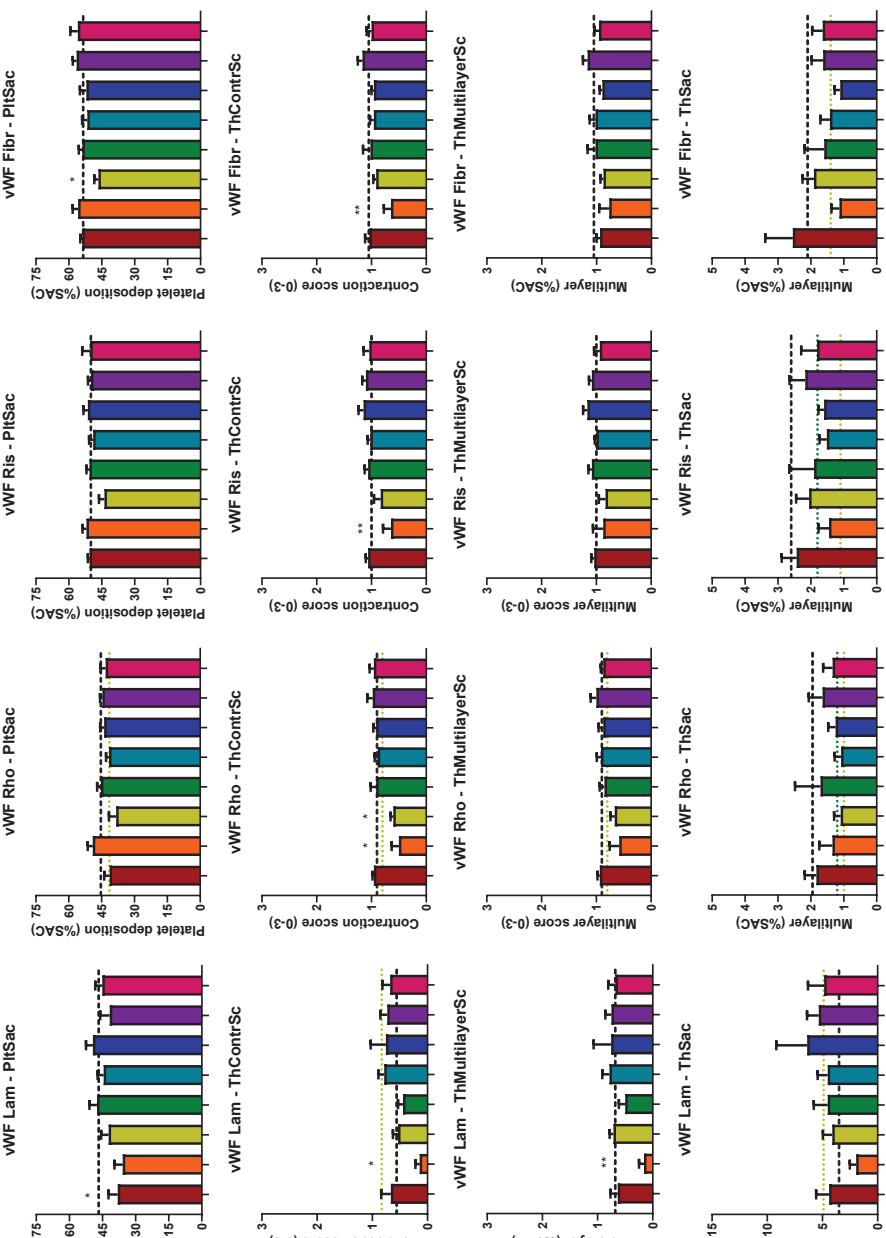

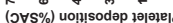
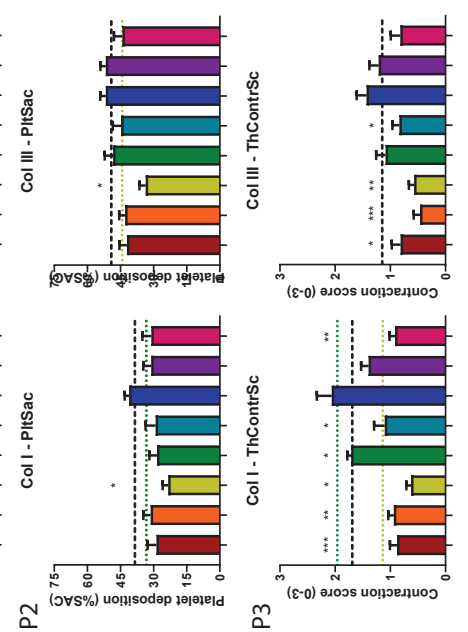
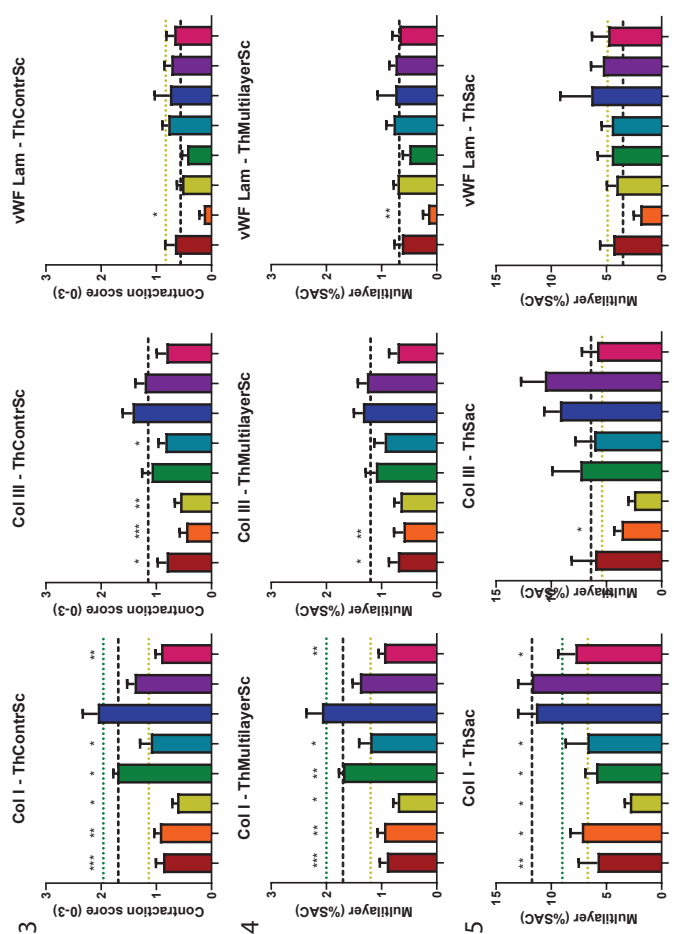

m

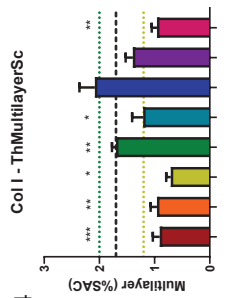

a

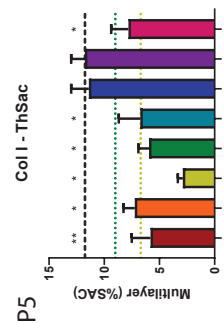



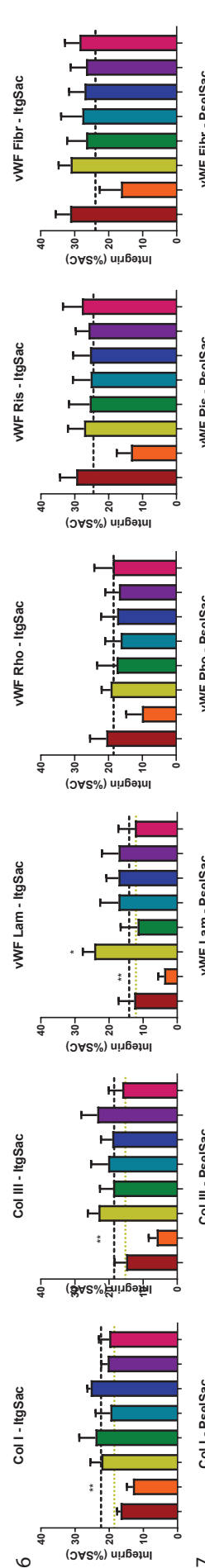

๑
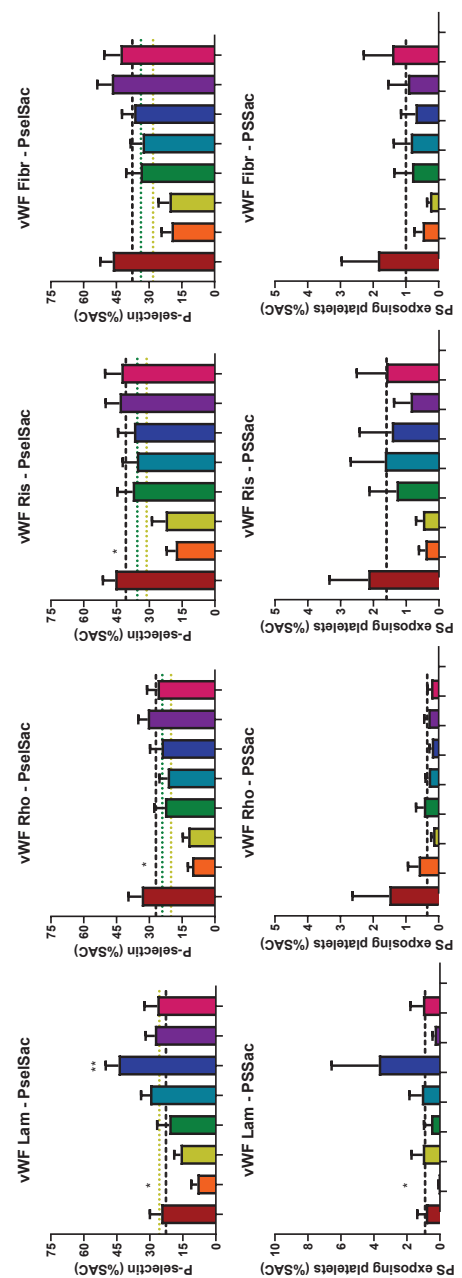

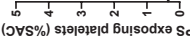
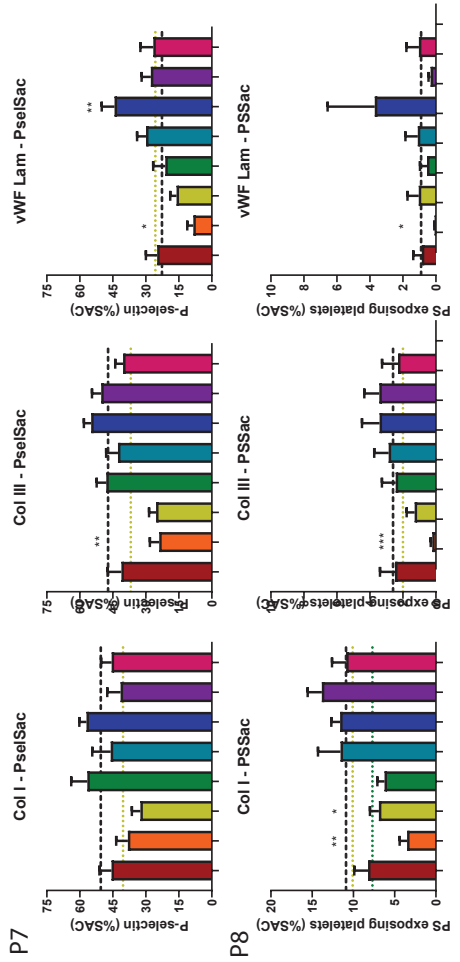

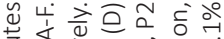

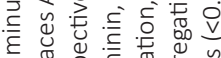

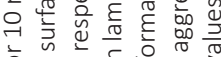

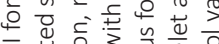

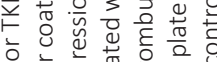

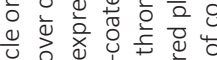

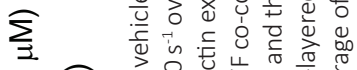

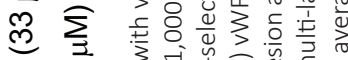

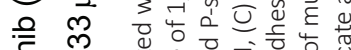

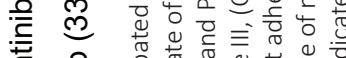

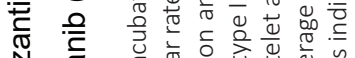
N $\frac{\pi}{0}$ ब

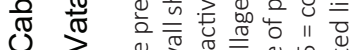

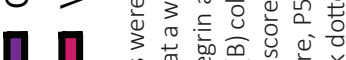

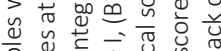

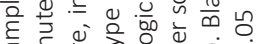

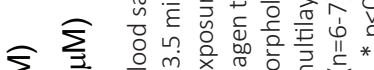

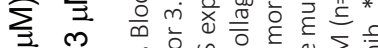
เి

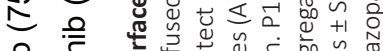

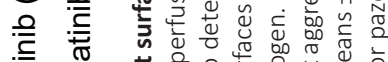

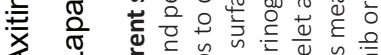

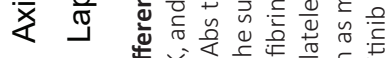

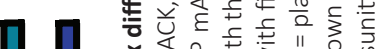

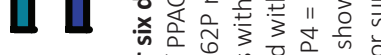

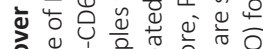

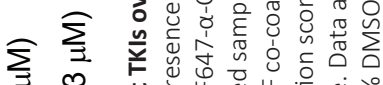

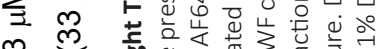

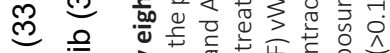

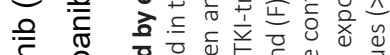

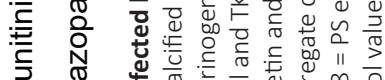
๙

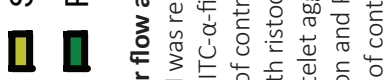

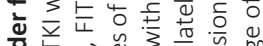

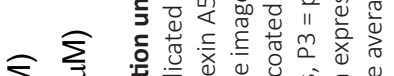

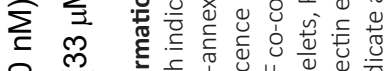

८

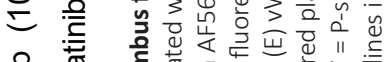

殸 贾 ๑ొ 1

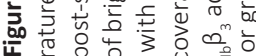
芭

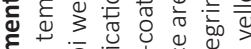

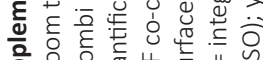

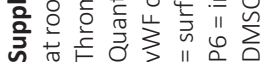



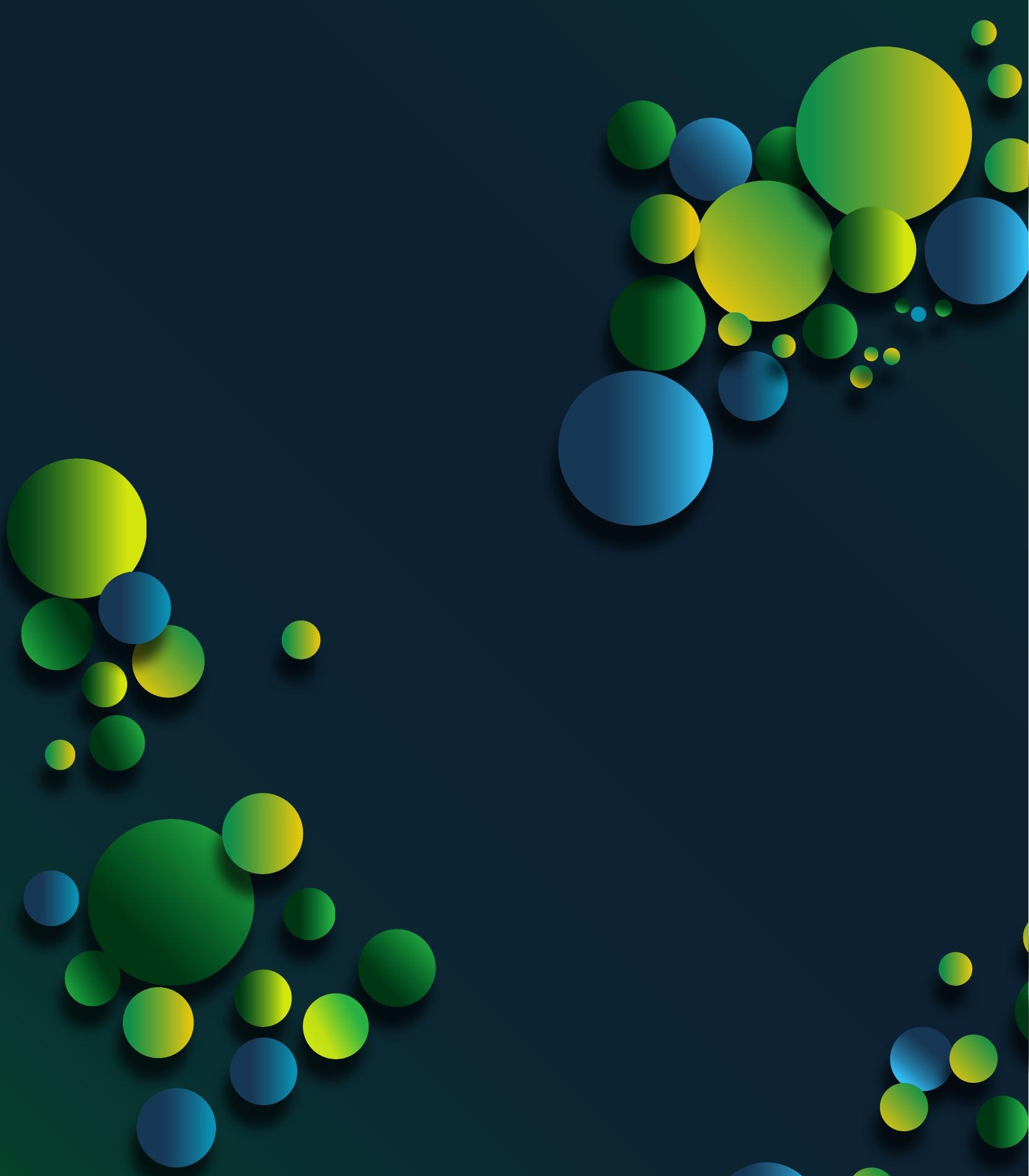


\section{Chapter 8}

General Discussion 
Since the beginning of this century, a new class of anti-cancer agents, tyrosine kinase inhibitors (TKIs), has been developed and approved for the treatment of several malignancies ${ }^{1,2}$. These TKIs inhibit growth factor receptors (tyrosine kinases, Table 8.1) involving angiogenesis and tumour development and thereby improving progression free survival (PFS) and overall survival $(\mathrm{OS})^{3}$. As most TKIs inhibit multiple protein tyrosine kinases (Table 8.1), they inevitably also affect other cell types. One of the side effects described for several TKIs is (mild) bleeding or thrombosis ${ }^{4}$. Platelets are well known for their involvement in haemostasis, and they also contain multiple protein tyrosine kinases (Syk, Src family kinases (SFK), Btk), which are essential for their activation. Hence, these TKIs may also affect platelet function. Therefore, the overall aim of this thesis was to provide more insights in the off-target effects of TKIs on platelet function (Chapter 1 ). In the next paragraphs of this chapter, the observations described throughout this thesis are discussed in relation to the current literature, as well as clinical implications and future perspectives are given. Altogether, the findings reported in this thesis may provide relevant insights for monitoring the bleeding risk in patients receiving TKI treatment by assessing quantitative and qualitive platelet traits, especially in the presence of anti-coagulant or anti-platelet drugs.

\section{Platelet signalling and TKIs}

It has been described that several TKIs, for different cancer types, inhibit platelet function upon treatment, which can contribute to the risk of bleeding ${ }^{5-7}$. Chapter 2 shows an overview

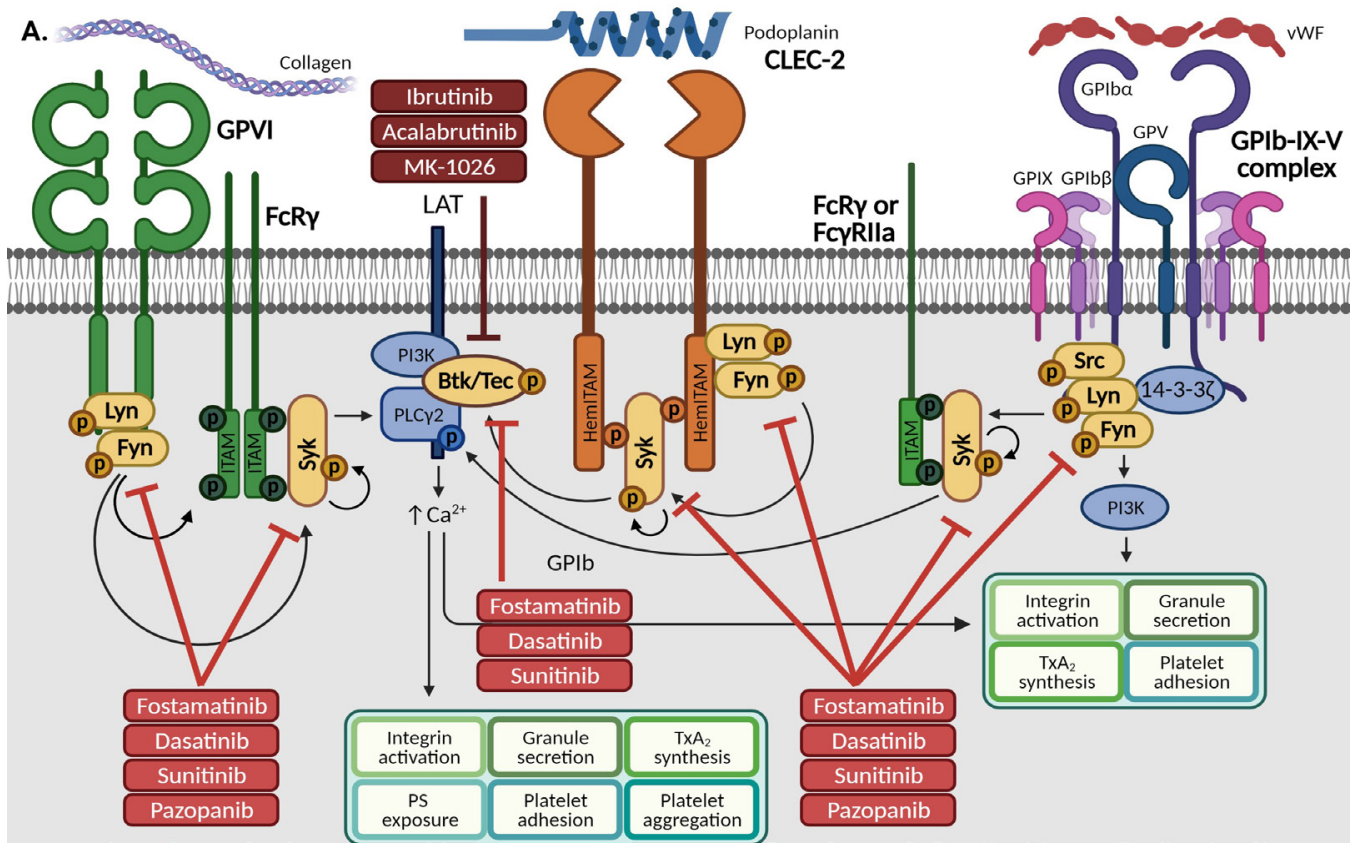

Figure 8.1. Overview of the tyrosine kinases expressed in platelet signalling (in yellow) downstream of multiple platelet receptors, resulting in different platelet responses. The intervention points of the TKIs (based on the $K d$ values described in Chapter 2) investigated in this thesis are indicated in red. 
of the currently used TKIs in cancer treatment and the effects on platelet function. Throughout the Chapters 3-7, we used in total 11 TKIs (acalabrutinib, axitinib, cabozantinib, dasatinib, fostamatinib, ibrutinib, lapatinib, MK-1026, pazopanib, sunitinib and vatalanib) to explore the effects on platelet function in vitro and in cancer patients. Table 8.1 gives an overview of the antiplatelet effects from different assays, that were observed after in vitro treatment with these 11 TKIs. Overall, the majority of inhibited responses were demonstrated to affect the GPVI-pathway, and for several TKIs (ibrutinib and fostamatinib), (also) effects upon CLEC-2 or GPIb stimulation

Bi.

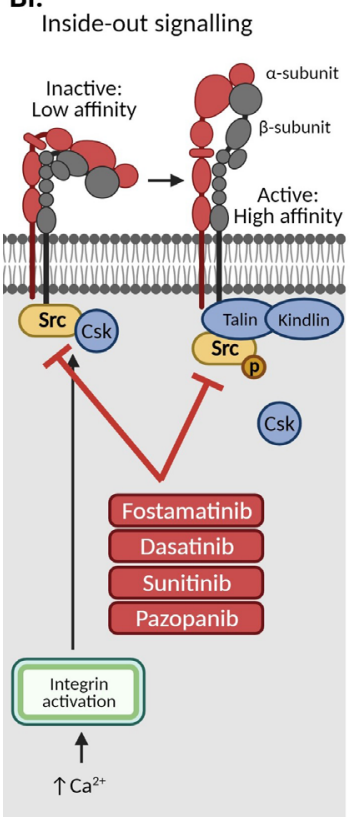

ii.

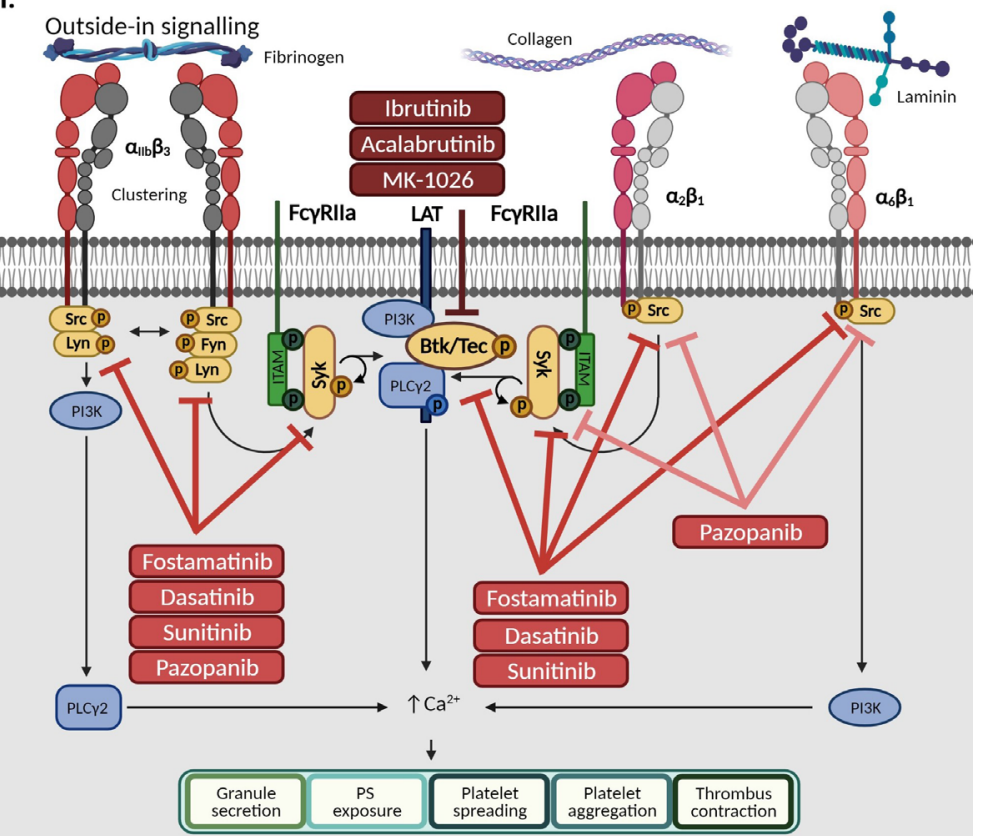

C.

GAS6 Fostamatinib

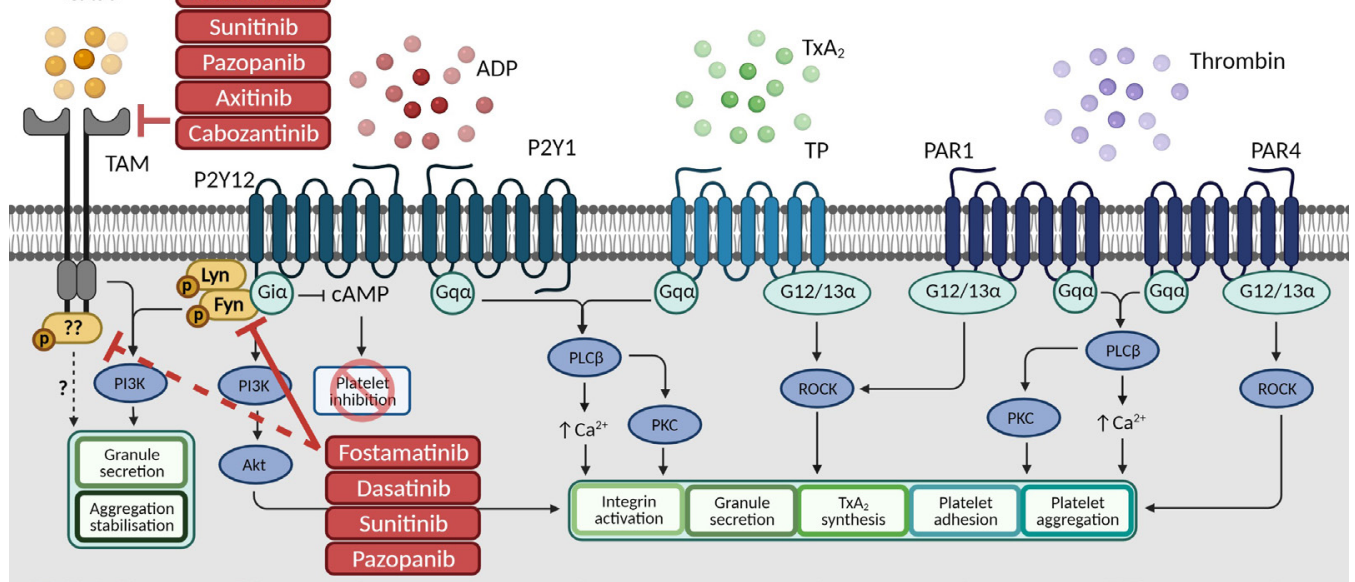

Figure 8.1. Continued. The intervention points of the TKIs (based on the Kd values described in Chapter 2) investigated in this thesis are indicated in red. 


\section{Chapter 8}

could be observed. Given the defined affinities in our review in combination with the results obtained for the 11 TKIs tested here, we visualised the proposed interference of these TKIs in the platelet signalling pathways (Figure 8.1).

In whole blood perfusion, effects on thrombus formation were mainly restricted to the glycoprotein (GP)VI-pathway as observed for dasatinib, sunitinib, pazopanib and vatalanib. Only ibrutinib and fostamatinib showed to be effective towards GPIb (combined with CLEC-2 or $\alpha_{6} \beta_{1}$ )induced thrombus formation (Table 8.1, Chapter 3, 4, 6 and 7). Ibrutinib and fostamatinib specifically inhibit Btk/Tec or Syk, respectively. Both proteins are important mediators in coupling cell surface receptors to downstream signalling events and are in close proximity in most signalling pathways ${ }^{8,}$ 9. This suggests that direct targeting of Btk or Syk in platelet signalling by TKIs is more effective to inhibit platelet function as compared to TKIs primary targeting growth factor receptors. Also, a difference can be observed between inhibition of Btk and Syk. Btk inhibition by ibrutinib mainly inhibits GPIb-associated thrombus formation, whereas Syk inhibition with fostamatinib shows to affect thrombus formation with GPIb, as well as GPVI. As binding and activation of Syk initiates downstream signalling involving Btk in GPVI, CLEC-2 and GPIb-signalling, this hints towards the concept that inhibition higher upstream in platelet signalling results in more efficient inhibition.

In isolated platelets almost all inhibitors more prominently inhibited GPVI-induced aggregation, except fostamatinib, axitinib and lapatinib. For fostamatinib this contrasts to the papers of Spalton et al. and Lhermusier et al. ${ }^{10,11}$, who observed complete inhibition of collagenand/or rhodocytin-induced platelet aggregation with already low concentrations of the active metabolite of fostamatinib. The only difference between these studies and the study in chapter 7 , is the incubation time of the platelets with the inhibitor. We pre-incubated for 10 minutes, while in the earlier studies platelets were incubated for 1-5 minutes with fostamatinib. As fostamatinib is a reversible inhibitor, the incubation time could be of importance, however, we did observe strong inhibition in whole blood perfusion experiments using 10 minutes pre-incubation of whole blood.

The lack of GPVI-induced aggregation for axitinib and lapatinib is in line with literature findings. Although axitinib is associated with bleeding ${ }^{7,12}$, no inhibition of platelet function has been reported. In about $15-20 \%$ of the patients, axitinib is described to induce mild (grade 1-2) thrombocytopenia ${ }^{13,14}$, and in rare cases $(<1 \%)$ severe ( $\geq$ grade 3 ) thrombocytopenia is reported ${ }^{15}$. For axitinib it is proposed that increased bleeding in these patients is caused by a decrease in platelet count rather than platelet inhibition. For lapatinib increased bleeding risk has not been reported and, furthermore, thrombus formation under flow (in vitro) was also unaffected ${ }^{16}$. Hence, these observations show that axitinib and lapatinib do not have off-target effects on platelets.

All other TKIs investigated in this thesis (acalabrutinib, cabozantinib, dasatinib, MK-1026, pazopanib, sunitinib and vatalanib) show most pronounced effects in platelets upon collagen stimulation. Inhibition in thrombus formation under flow was restricted to GPVI-induced 
activation for pazopanib, sunitinib, dasatinib and vatalanib. This suggests that the GPVI pathway is most sensitive for inhibition with TKIs. GPVI, GPIb-IX-V and CLEC-2 are most potent receptors for thrombus formation at high-shear stress conditions ${ }^{17}$. They share several common tyrosine kinases (Fyn, Lyn, Syk, Btk, etc) in signal transduction in platelets ${ }^{17}{ }^{18}$. As TKIs are able to affect multiple of these tyrosine kinases, it is expected that more pathways should be inhibited. However, a possible explanation for the restriction of effects to the GPVI pathway could be the amount of copy numbers present in platelets. Of these receptors, GPIIblla (integrin $\alpha_{11} \beta_{3}$ ) and GPIb are the most abundantly expressed receptor on platelets with around 80,000 and 25,000 copies, respectively ${ }^{19-21}$. GPVI and CLEC-2 receptors are far less expressed with only 6,000-10,000 and 2,000 copies reported, respectively ${ }^{19}, 22,23$. Therefore, it could be expected that GPVI and CLEC-2 are relatively more affected as compared to GPIb as less receptors (lower copy number) remain active. Signalling pathways of receptors with a higher expression, such as GPIIblla and GPIb, might still be partially functional as more receptors are available, making it more difficult to observe inhibition. Moreover, it is indicated by Spalton et al. ${ }^{10}$ that GPVI is more dependent on Src family kinase (SFK) phosphorylation and FCYR signalling, whereas the CLEC-2 pathway is more reliant on Syk phosphorylation. Together this encourages that TKIs have probably a higher affinity for SFKs rather that Syk, resulting in more specific inhibition of the GPVI pathway.

\section{Plasma protein binding of TKIs influencing drug availability and platelet inhibition}

In (almost) all chapters of this thesis inhibition of platelet traits, qualitative and/or quantitative, by different TKIs is the central point of view. Although in vitro effects were present in almost all conditions tested, inhibition by TKIs was more pronounced in isolated platelets as compared to the presence of plasma (platelet-rich plasma or whole blood) (Table 8.1). This meant that a higher dose of a TKI was often needed to elicit similar inhibition in PRP or whole blood as compared to washed platelets. Strikingly, pazopanib and cabozantinib prominently inhibited aggregation of washed platelets to several agonists, while in PRP this response was unaffected (Table 8.1). This phenomenon is caused by high plasma binding of the TKIs $(>95 \%)^{24-27}$. In patients with TKI treatment, the bioavailability of the drug is likely to be influenced by this plasma binding with implications for drug toxicity and response ${ }^{28}$. This shows the importance of the comparison between in vitro testing of these agents in presence or absence of plasma, as well as measuring effects present in patients receiving treatment. For clinical implications it is might point to adapting dosing regimens based on the pharmacokinetic/dynamic characteristics of $\mathrm{TKIs}^{29}$ or more ideally by monitoring the unbound plasma concentration of a TKI during treatment ${ }^{30}$. For example, we measured levels of sunitinib and its metabolite in plasma, serum and isolated platelets at 2 and 4 weeks on treatment in patients with a 4/2 scheme (4 weeks dosing, 2 weeks rest) in Chapter 5. We showed that in plasma and serum, the unbound concentrations were comparable for 


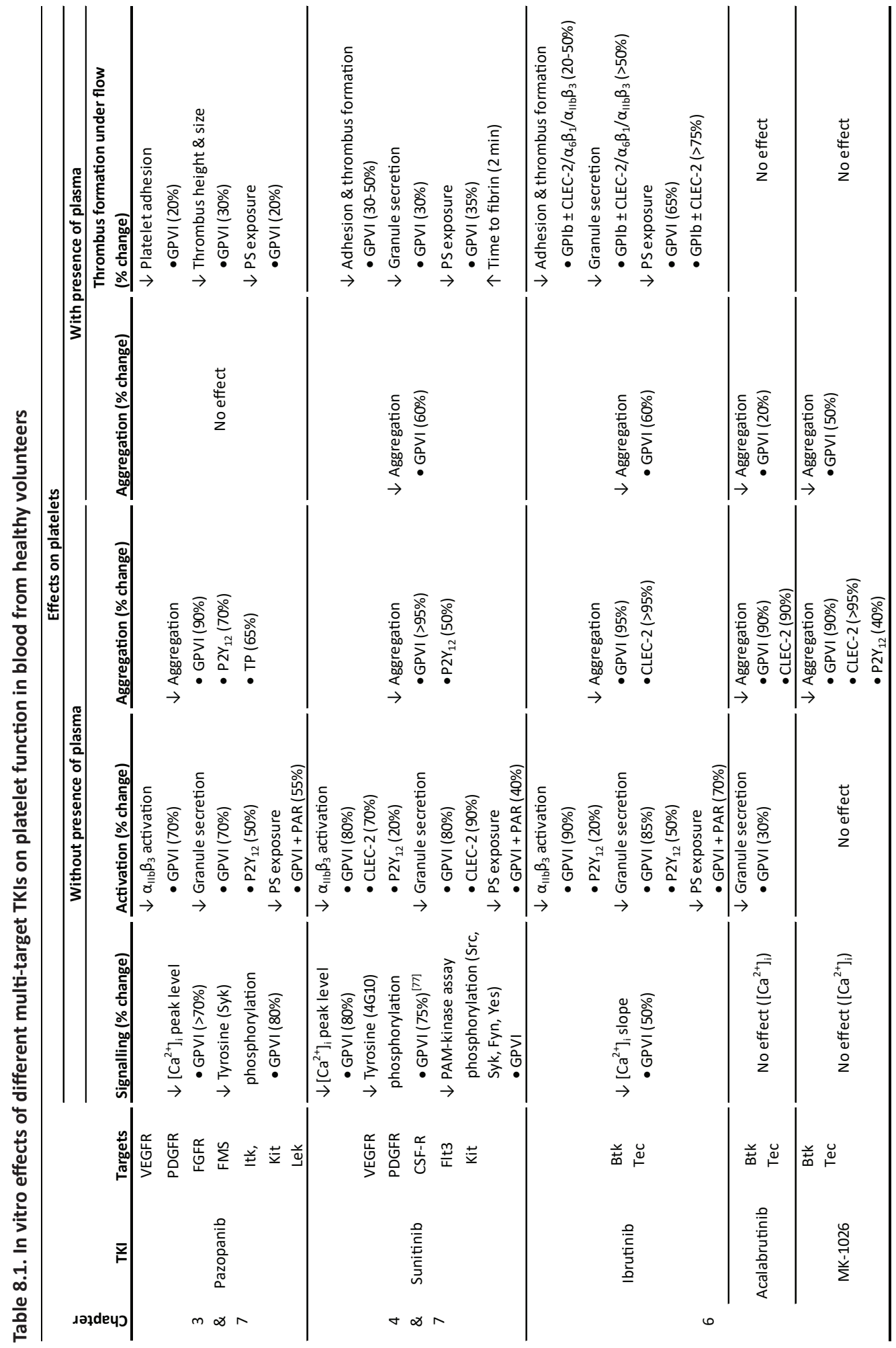




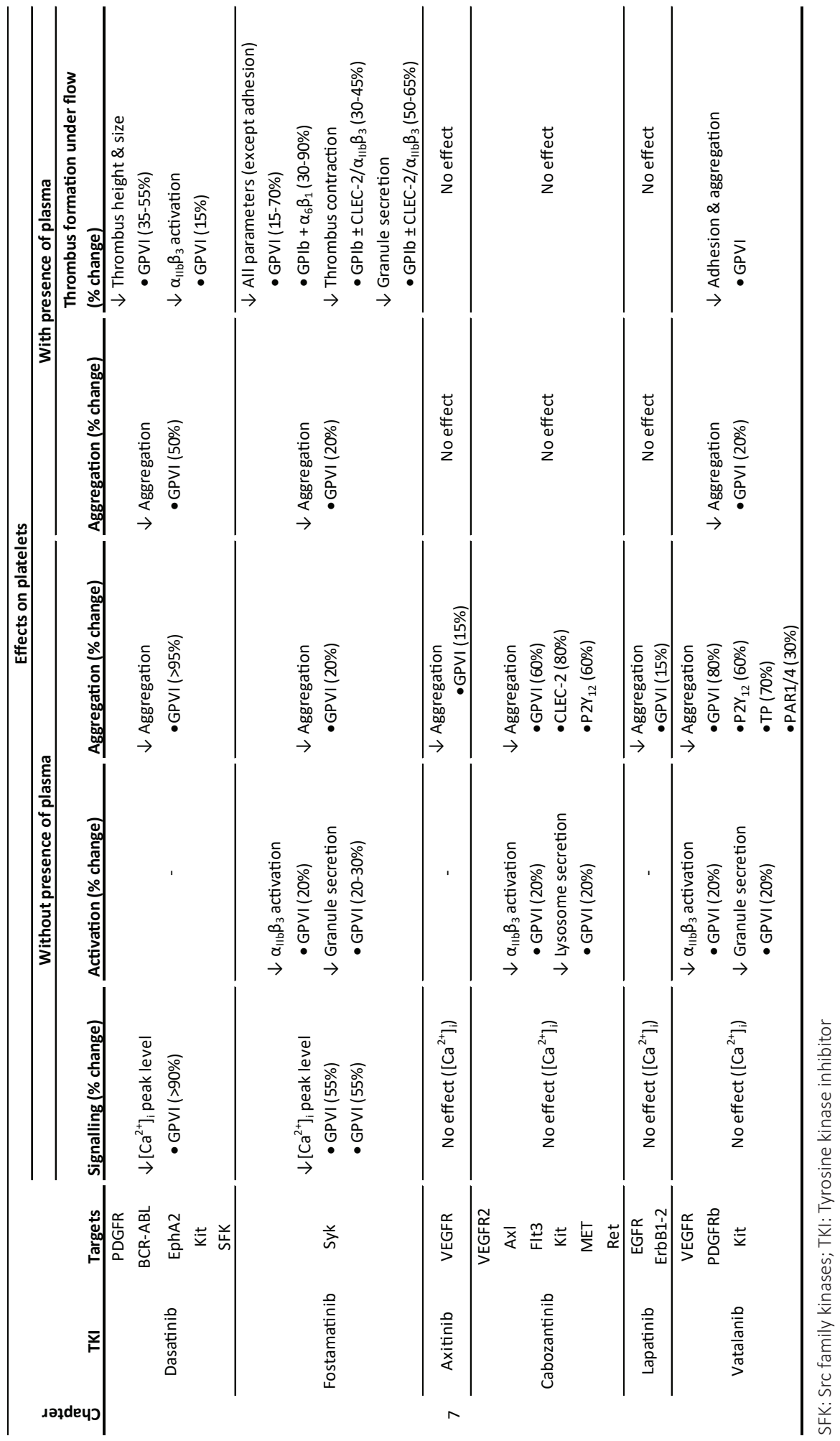


sunitinib and its metabolite at both timepoints, however, a remarkable increase of metabolite concentration was observed in isolated platelets as compared to sunitinib concentration. This suggested a higher uptake of the active form of sunitinib by platelets in their granules, though both compounds were equally present in plasma. Therefore, it might also be of interest to not only monitor plasma concentrations at 2 of 4 weeks on treatment (dependent on treatment schedule) ${ }^{31}$, but also measure the uptake of (active) compound by platelets as this might predict the platelet inhibitory effects.

\section{Involvement of quantitative and quantitative platelet traits in bleeding tendency in patients}

In the review in Chapter 2, 11 out of almost 40 TKIs are reported with an increased risk of bleeding or bleeding events. Given the key role of platelets in haemostasis, both platelet count and platelet function are relevant to prevent bleeding ${ }^{32,33}$. In Chapters 3 and 5, we have shown that not only platelet function, but also platelet count is already decreased after 2 weeks after pazopanib or sunitinib treatment, as compared to pre-treatment (Table 8.2). Although TKIs are known to be associated with platelet dysfunction ${ }^{6,34}$, impaired platelet function is not always found to be the cause of increased bleeding tendency ${ }^{35,36}$. For multiple TKIs, including dasatinib (Chapter 7), an association is found with drug-induced immune thrombocytopenia, defined as a platelet count below $50 \times 10^{9} / \mathrm{L}^{37-39}$, resulting in an increased risk of bleeding. While most of the patients included in Chapters 3 and 5 did not show thrombocytopenia, we did observe an overall decrease in platelet count upon pazopanib or sunitinib treatment (Table 8.2). This change in platelet count correlated with the inhibition of platelet function after sunitinib treatment, as well as with the plasma concentration of the metabolite of sunitinib (Chapter 5). This point to the idea that platelets are exposed to significant levels of sunitinib resulting in (mild) anti-platelet effects. Alternatively, sunitinib may act on the megakaryocytes, thereby decreasing platelet count and possibly also affect platelet function. As plasma concentration and plasma protein binding of sunitinib (or its metabolite) could be of importance in toxicity and response to a TKI treatment ${ }^{30}$, this pointed toward a monitoring tool for off-target effects and/or progression-free survival.

Whilst several studies propose that TKI-associated platelet dysfunction does not cause bleeding 35,36 , almost all the patients included in Chapter 3, 5 and 6 did report (mild) bleeding episodes upon TKI treatment in the absence of thrombocytopenia (Table 8.2). This may imply that pharmacological platelet inhibition does play a role in the increase in bleeding risk. In agreement, we see impairment of GPVI-induced platelet activation, aggregation or thrombus formation upon treatment in vitro and/or in patients, some with additional effects on CLEC-2- or GPIb-induced platelet responses. It has been reported that targeting GPVI via GPVI inhibitors or GPVI depletion in mice or patients does not (significantly) impair haemostasis ${ }^{40-45}$. However, a combined deficiency of GPVI and CLEC-2 in mice does show impaired haemostasis ${ }^{43}$. These 


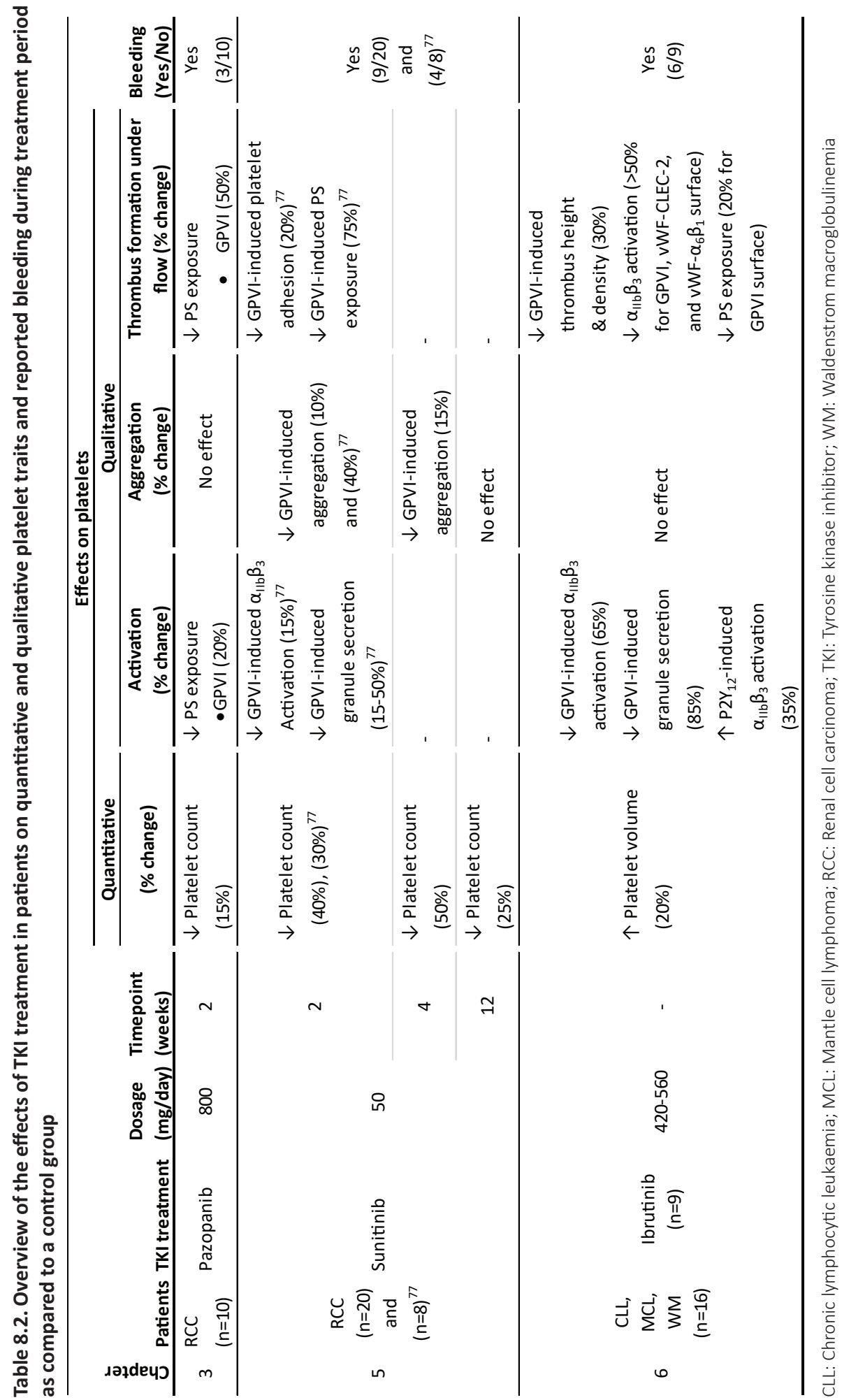




\section{Chapter 8}

studies mainly determined the contribution of platelet GPVI- and CLEC-2 receptors themselves in haemostasis ${ }^{40,43}$. However, in case of several TKIs, the inhibition in platelet signalling underneath these receptors is described to be via SFKs (Fyn, Lyn) or Syk ${ }^{11,46-49}$. As the GPVI, CLEC-2 and GPIb pathways all require SFKs and/or Syk in its signalling, it overall suggests that multi-target TKIs can elicit combined inhibition of GPVI plus CLEC-2 or possibly GPIb signalling pathways. This could be more potent than targeting solely one receptor and, hence, relevant for bleeding tendency. In combination with a decrease in platelet count, this might even further increase the bleeding risk.

As cancer patients often have a cardiovascular history ${ }^{50,51}$, anti-platelet or anti-coagulant drugs are prescribed to prevent recurrent cardiovascular events. Anti-platelet therapies, such as clopidogrel and aspirin, are reported to be associated with an increased risk of bleeding ${ }^{52-54}$. As TKIs are described to increase this risk as well, in Chapter 5, we investigated the synergistic effects of sunitinib and aspirin as anti-platelet medication on thrombus formation and fibrin formation to elucidate the impact on haemostasis. We demonstrated that sunitinib suppressed procoagulant activity and prolonged the time to fibrin formation, which was aggravated by aspirin treatment. Therefore, a combination of TKIs and anti-platelet therapies may be expected to increase the frequency or grade of bleeding. Though such dual treatment of TKI with anti-platelet or anticoagulant drugs could be increasing the bleeding risk, this might also be beneficial for the patients. It has been shown that anti-platelet therapies have anti-tumour effects in hepatocellular and colorectal cancer ${ }^{55}{ }^{56}$. Hence a combination of TKI with anti-platelet therapy could enhance the anti-tumour effects. For example, erlotinib together with aspirin is described to enhance sensitivity of the cancer as compared to aspirin alone, thereby increasing the anti-cancer activity $^{57}$. The application of anti-platelet drugs is even more favourable, as it was shown that low doses of aspirin could be used as treatment for off-target effects, such as skin rash caused by erlotinib-treatment ${ }^{58}$. Additionally, for some TKIs, anti-platelet treatments can be useful as nilotinib is associated with an increased risk of thrombosis ${ }^{59}$. Yet, not all anti-platelet drugs show a positive correlation in combination with TKI treatment. The study of Haoula et al. ${ }^{60}$ highlights the importance of drug-drug interactions between clopidogrel and other anticoagulants in combination with TKIs. This urges for better understanding of dual treatment with TKIs and antiplatelet or anti-coagulant therapies to monitor patients.

Overall, we showed that multiple TKIs inhibit platelet function via different pathways and that this combination may contribute to the reported bleeding risk in patients. In addition, for other TKIs inhibiting the GPVI-induced platelet function together with a decrease in platelet count, as seen with pazopanib and sunitinib, might also explain the mild bleeding tendency observed during treatment. Hence, the combination of the inhibitory effects on platelet traits together with anti-platelet or anti-coagulant drugs urges for better understanding and possibly more strict monitoring of patients as this may further increase the risk of bleeding. 


\section{Clinical implications}

Platelets not only have an important role in haemostasis, they have also been described to be implicated in tumour development and growth ${ }^{61-64}$. Platelets are known to take up various pro- and antiangiogenic proteins into their $\alpha$-granules ${ }^{61,62,65}$. These granules can be secreted upon platelet activation by activating factors expressed or secreted by tumour vasculature such as thrombin and ADP. In turn, the secreted proangiogenic factors can stimulate tumour growth and metastasis by stimulating angiogenesis ${ }^{61,66}$, improving vasculature integrity ${ }^{67,68}$ and inducing resistance to therapy ${ }^{69,70}$. Due to this promoting role of platelets in tumour angiogenesis and progression, as well as the correlation with reduced survival, platelets are an interesting target for cancer therapy. Although numerous drugs have been developed to target platelet receptors ${ }^{71}$, limitations for implications occur due the role of platelets in haemostasis associated with increased bleeding tendency ${ }^{52-54}$. Yet anti-platelet treatment have been described to reduce tumour angiogenesis, growth and progression ${ }^{72,73}$ by direct inhibition of the tumour or via indirect via inhibition of platelet secretion, thereby decreasing proangiogenic protein concentrations in the tumour environment ${ }^{74}$. Hence, the TKIs investigated in this thesis could also indirectly affect tumour growth via the inhibiting effects on platelet function, as we did observe inhibition of platelet activation and/or secretion upon GPVI stimulation with acalabrutinib, cabozantinib, fostamatinib, ibrutinib, pazopanib, sunitinib and vatalanib in isolated platelets. However, the principle of platelet-mediated anti-cancer effect of TKIs needs to be further investigated. Platelet inhibition remains contradictory and needs to be balanced, as it can improve cancer prognosis, as well as increase the risk of bleeding. In line with the inhibition of platelet function with TKIs observed throughout this thesis, one might argue that monitoring patients is needed to profit from the anti-tumour effects and keep the risk of undesirable bleeding to a minimum.

In addition, platelet production can be (greatly) enhanced by malignant (solid) tumours ${ }^{62}$, 75. An increased platelet count or thrombocytosis (platelet count of $>400 \times 10^{9} / \mathrm{L}$ ) is recognized as a predictor of poor prognosis in patients with different cancer types ${ }^{75,76}$. As platelets are able to take up all sorts of compounds into their granules, it is not unlikely for anti-cancer agents to be sequestered as well. The study of Sabrkhany et al. ${ }^{77}$ showed that platelets take up sunitinib in their granules in vitro and in patients. In turn, we showed in Chapter 5 that both sunitinib and its metabolite were present in isolated platelet of sunitinib-treated patients and that platelet function is impaired. Besides high plasma binding of anti-cancer or anti-platelet agents, the ability of platelets to sequester these compounds might influence the bioavailability for the tumour, especially in thrombocytosis, i.e. increased amount of platelets. This might result in reduced inhibition of tumour growth as a lower concentration would be circulating in the blood. Also, thrombocytosis might not only be a consequence of cancer, it might be a paraneoplastic abnormality that is associated with a vicious cycle of promoting tumour cells, platelet activation and stimulating thrombocytosis ${ }^{75}$. Therefore, reducing the thrombocytosis before starting 
treatment by interfering with this feedback-loop could be relevant. This could be accomplished via inhibition of platelet function with anti-platelet agents or heparins, however this could result in bleeding implications ${ }^{78,79}$. Another approach to intervene with tumour growth and metastasis is inhibition of interleukin-6 (IL-6) by the IL-6 antibody siltuximab. This will deplete the increased growth factor levels present and additionally decrease platelet count ${ }^{80}$. Clinical trials with ovarian cancer, renal cell carcinoma and castration resistant prostate cancer already show beneficial effects ${ }^{80-82}$.

\section{Clinical significance for renal cell carcinoma patients}

The TKIs used for treatment of patients with metastatic renal cell carcinoma (mRCC), i.e. sunitinib and pazopanib, have been the focus of Chapters 3, 4 and 5 of this thesis. Furthermore, we also studied the effects of cabozantinib, used mainly as 2 nd or 3rd line treatment for mRCC patients, and axitinib, combined with pembrolizumab in first line $\mathrm{mRCC}^{83,84}$, on platelet functions in Chapter 7. In the Netherlands, good risk mRCC patients are treated with a TKI when metastases have occurred. Patients often have a large tumour thrombus in the renal vein, which means that local surgery is no longer possible. However, this is usually no consideration to start anticoagulation therapy. When started with $50 \mathrm{mg} /$ day sunitinib, systemic concentrations of sunitinib should be 50-100 ng/mL ${ }^{85}$. However, for a substantial group of patients, this dosing schedule may result in much higher peak levels, resulting in toxicity ${ }^{86}$. In daily practice, sunitinib levels (steady state) in serum could be determined if patients experience serious side effects and if the levels are too high ( $>100 \mathrm{ng} / \mathrm{mL}^{31}$ ), the treatment dose could be adjusted accordingly. In addition, sunitinib levels could also be determined if patients have no side effects at all, as the sunitinib level is probably too low $\left(<50 \mathrm{ng} / \mathrm{mL}^{31}\right)$.

Hamilton et al. ${ }^{87}$ have shown that the decrease in platelet count upon sunitinib treatment may serve as a prognostic value with regard to tumour development. In Chapter 5, we have demonstrated that this reduction in platelet count also coincides with a reduction in platelet function as well as sunitinib levels in plasma and serum. Together, this implies that both qualitative and quantitative platelet traits may serve as a monitoring tool with regards to sunitinib treatment in mRCC patients. Furthermore, similar observations have been reported for neutrophil-tolymphocyte ratio (NLR) and platelet-to-lymphocyte (PLR) ratio. A NLR greater than 1.9 but below 3 and PLR over 160 are described to be favourable prognostic factors ${ }^{88,89}$. Also, an increase in eosinophils have been reported to be a beneficial factor upon therapy in patients with renal cell carcinoma upon treatment ${ }^{90,91}$.

Also relevant for $\mathrm{mRCC}$, although no longer used, is the classification of metastatic disease into groups with regards to overall survival, according to the Memorial Sloan-Kettering Cancer Centre (MSKCC)/Motzer citeria92; good, intermediate and poor risk. Currently, a switch has been made to the International Metastatic RCC Database Consortium (IMDC) criteria to make group 
classification. For this purpose, 6 parts of pre-treatment are scored, with one point per item, looking back one year before starting systemic treatment: Time from diagnosis to treatment initiation $<1$ year, Karnofsky performance status (KPS) $<80 \%$, haemoglobin $(\mathrm{Hb})$ concentration $<12 \mathrm{~g} / \mathrm{L}$, serum calcium concentration > upper limit of normal (ULN), neutrophil count $>U L N$ and platelets count> ULN ${ }^{93}$. With no points, the patient has a good risk for survival, at 1-2 points intermediate risk and from 3 on the risk is poor. For the good risk patients, it appeared that sunitinib was superior to the immuno-agents nivolumab/ipilimumab, and was equivalent to pembrolizumab plus axitinib ${ }^{94,95}$. Therefore, sunitinib has become a mainstay not only for firstline treatment in this particular risk group, but also in second line treatment after nivolumab/ ipilimumab. The results of the present thesis with regard to the effects of sunitinib on platelet count and function, are thus in agreement with the incorporation of platelets into this IMDC classification.

\section{Future perspectives}

To summarise, this thesis increased the knowledge of how TKIs can influence platelet function and contribute to reported bleeding events. In brief, in vitro experiments throughout this thesis demonstrate that impaired platelet function is more pronounced in isolated platelets as compared to whole blood perfusion, mainly affecting GPVI-induced platelet activation in presence of plasma. Only ibrutinib and fostamatinib showed (additional) inhibition of CLEC-2 and/or GPIb-induced platelet activation and aggregation. In addition, the observed suppression of aggregation and thrombus formation with sunitinib was aggravated by anti-platelet (aspirin) therapy. Our studies involving patients demonstrated that pazopanib or sunitinib treatment slightly decreased platelet count together with (limited) effects on the qualitative platelet traits, whereas for patients treated with ibrutinib impaired collagen-, rhodocytin- and vWF-induced aggregation and thrombus formation was observed. Moreover, we showed that for sunitinib the inhibition in quantitative and qualitative platelet traits could be correlated with the (effective) sunitinib levels in patients. Altogether, the inhibition of multiple platelet activation pathways, the reduction in platelet count, or TKI treatment combined with antiplatelet agents might contribute or even increase the reported bleeding tendencies of these TKIs. Therefore, patients should be monitored by measuring platelet function or (active) compound levels in serum, plasma or isolated platelets 2-4 weeks after start treatment or additionally in case of changes like dose reduction, reoccurrence of side effects or altering treatment schedule. Though, more research is needed to determine effects on sunitinib concentration in long term and whether possible effects extinguish over time, as our study in Chapter 6 is limited to follow-up period of maximal 12 weeks and limited in patient numbers due to dose reduction or discontinuation of medication (upon decease).

To further develop the findings of this thesis towards better and improved patient care, 


\section{Chapter 8}

more research is needed. Our patient study with sunitinib showed the correlation between quantitative and qualitative platelet traits with (active) sunitinib levels. Due a limited number of patients, no correlation could be observed between these parameters and bleeding and/or progression. Therefore, it would be interesting to investigate whether changes in platelet traits or concentration of unbound sunitinib (or other TKIs) in plasma or isolated platelets could predict response to therapy or bleeding in a larger cohort. This knowledge might lead to a way to follow patients more closely to prevent bleeding or monitor progression. As we observed aggravated effects on platelet function of sunitinib together with aspirin, more research into the combined anti-platelet effects of different anti-platelet or anti-coagulant drugs with TKIs is necessary. Especially patients receiving both anti-cancer and anti-platelet therapies could be at increased risk for bleeding.

The application of TKIs in treatment of other diseases, e.g. thrombosis, would also be fascinating to study. TKIs already have inhibiting effects on platelets mainly via the GPVI pathway, therefore, these drugs might be possible to use as anti-platelet drugs in (deep vein) thrombosis, which is also suggested recently by other studies ${ }^{96,97}$. These TKIs are already approved in the clinic, ergo easier to test in other diseases involving thrombosis or thrombo-inflammation, in a short period of time as side effects are already reported. Additionally, due to the relative high affinity described for tyrosine kinases, relatively low therapeutic concentrations of TKI could be able to selectively inhibit platelet function without possibly increasing bleeding risk.

\section{References}

1. Jeong W, Doroshow JH, Kummar S. United States Food and Drug Administration approved oral kinase inhibitors for the treatment of malignancies. Curr Probl Cancer. 2013; 37: 110-44.

2. Roskoski R, Jr. Properties of FDA-approved small molecule protein kinase inhibitors: A 2020 update. Pharmacol Res. 2020; 152: 104609.

3. Gotink KJ, Verheul HM. Anti-angiogenic tyrosine kinase inhibitors: what is their mechanism of action? Angiogenesis. 2010; 13: 1-14.

4. Tullemans BME, Heemskerk JWM, Kuijpers MJE. Acquired platelet antagonism: off-target antiplatelet effects of malignancy treatment with tyrosine kinase inhibitors. J Thromb Haemost. 2018; 16: 168699.

5. Deb S, Boknäs N, Sjöström C, Tharmakulanathan A, Lotfi K, Ramström S. Varying effects of tyrosine kinase inhibitors on platelet function-A need for individualized CML treatment to minimize the risk for hemostatic and thrombotic complications? Cancer Med. 2020; 9: 313-23.

6. Quintás-Cardama A, Han X, Kantarjian H, Cortes J. Tyrosine kinase inhibitor-induced platelet dysfunction in patients with chronic myeloid leukemia. Blood. 2009; 114: 261-3.

7. Abdul Sater H. Receptor Tyrosine Kinases in Human Platelets: A Review of Expression, Function and Inhibition in Relation to the Risk of Bleeding or Thrombocytopenia from Phase I through Phase III Trials. J Cancer Prev Curr Res. 2017; 8.

8. Hsu J, Zhang J, Kitson C, et al. Development of a pharmacodynamic assay based on PLCY2 phosphorylation for quantifying spleen tyrosine kinase (SYK)-Bruton's tyrosine kinase (BTK) signaling. J Biomol Screen. 2013; 18: 890-8.

9. Tan SL, Liao C, Lucas MC, Stevenson C, DeMartino JA. Targeting the SYK-BTK axis for the treatment of immunological and hematological disorders: recent progress and therapeutic perspectives. Pharmacol Ther. 2013; 138: 294-309. 
10. Spalton JC, Mori J, Pollitt AY, Hughes CE, Eble JA, Watson SP. The novel Syk inhibitor R406 reveals mechanistic differences in the initiation of GPVI and CLEC-2 signaling in platelets. J Thromb Haemost. 2009; 7: 1192-9.

11. Lhermusier T, van Rottem J, Garcia C, et al. The Syk-kinase inhibitor R406 impairs platelet activation and monocyte tissue factor expression triggered by heparin-PF4 complex directed antibodies. J Thromb Haemost. 2011; 9: 2067-76.

12. Gunnarsson O, Pfanzelter NR, Cohen RB, Keefe SM. Evaluating the safety and efficacy of axitinib in the treatment of advanced renal cell carcinoma. Cancer Manag Res. 2015; 7: 65-73.

13. Rini BI. Targeted therapy for patients with renal-cell carcinoma. Lancet Oncol. 2011; 12: 1085-7.

14. Rini BI. Metastatic renal cell carcinoma: many treatment options, one patient. J Clin Oncol. 2009; 27: 3225-34.

15. Koksal UI, Goffin J, Lewis B, et al. A Case Report with Severe Thrombocytopenia Induced by Axitinib. Case Rep Hematol. 2020; 2020: 7520783.

16. Li R, Grosser T, Diamond SL. Microfluidic whole blood testing of platelet response to pharmacological agents. Platelets. 2017; 28: 457-62.

17. van der Meijden PEJ, Heemskerk JWM. Platelet biology and functions: new concepts and clinical perspectives. Nat Rev Cardiol. 2019; 16: 166-79.

18. Estevez B, Du X. New Concepts and Mechanisms of Platelet Activation Signaling. Physiology (Bethesda). 2017; 32: 162-77.

19. Burkhart JM, Vaudel M, Gambaryan S, et al. The first comprehensive and quantitative analysis of human platelet protein composition allows the comparative analysis of structural and functional pathways. Blood. 2012; 120: e73-82.

20. Cosemans JM, Iserbyt BF, Deckmyn $\mathrm{H}$, Heemskerk JW. Multiple ways to switch platelet integrins on and off. J Thromb Haemost. 2008; 6: 1253-61.

21. Varga-Szabo D, Pleines I, Nieswandt B. Cell adhesion mechanisms in platelets. Arterioscler Thromb Vasc Biol. 2008; 28: 403-12.

22. Saboor M, Ayub Q, llyas S, Moinuddin. Platelet receptors; an instrumental of platelet physiology. Pak J Med Sci. 2013; 29: 891-6.

23. Gitz E, Pollitt AY, Gitz-Francois JJ, et al. CLEC-2 expression is maintained on activated platelets and on platelet microparticles. Blood. 2014; 124: 2262-70.

24. O'Brien Z, Moghaddam MF. A Systematic Analysis of Physicochemical and ADME Properties of All Small Molecule Kinase Inhibitors Approved by US FDA from January 2001 to October 2015. Curr Med Chem. 2017; 24: 3159-84.

25. Podoll T, Pearson PG, Evarts J, et al. Bioavailability, Biotransformation, and Excretion of the Covalent Bruton Tyrosine Kinase Inhibitor Acalabrutinib in Rats, Dogs, and Humans. Drug Metab Dispos. 2019; 47: 145-54.

26. McAdoo SP, Tam FW. Fostamatinib Disodium. Drugs Future. 2011; 36: 273.

27. Wang X, Owzar K, Gupta P, et al. Vatalanib population pharmacokinetics in patients with myelodysplastic syndrome: CALGB 10105 (Alliance). Br J Clin Pharmacol. 2014; 78: 1005-13.

28. Li J, Brahmer J, Messersmith W, Hidalgo M, Baker SD. Binding of gefitinib, an inhibitor of epidermal growth factor receptor-tyrosine kinase, to plasma proteins and blood cells: in vitro and in cancer patients. Invest New Drugs. 2006; 24: 291-7.

29. Roberts JA, Pea F, Lipman J. The clinical relevance of plasma protein binding changes. Clin Pharmacokinet. 2013; 52: 1-8.

30. Wu J, Lorusso PM, Matherly LH, Li J. Implications of plasma protein binding for pharmacokinetics and pharmacodynamics of the $\gamma$-secretase inhibitor RO4929097. Clin Cancer Res. 2012; 18: 2066-79.

31. Yu H, Steeghs $N$, Nijenhuis CM, Schellens JH, Beijnen JH, Huitema AD. Practical guidelines for therapeutic drug monitoring of anticancer tyrosine kinase inhibitors: focus on the pharmacokinetic targets. Clin Pharmacokinet. 2014; 53: 305-25.

32. Gieger C, Radhakrishnan A, Cvejic A, et al. New gene functions in megakaryopoiesis and platelet formation. Nature. 2011; 480: 201-8.

33. Nugent $D$, Kunicki T. Platelet genomics: the role of platelet size and number in health and disease. Platelets. 2017; 28: 27-33.

34. Neelakantan P, Marin D, Laffan M, Goldman J, Apperley J, Milojkovic D. Platelet dysfunction associated 
with ponatinib, a new pan BCR-ABL inhibitor with efficacy for chronic myeloid leukemia resistant to multiple tyrosine kinase inhibitor therapy. Haematologica. 2012; 97: 1444.

35. Sener Y, Okay M, Aydin S, Buyukasik Y, Akbiyik F, Dikmen ZG. TKI-Related Platelet Dysfunction Does Not Correlate With Bleeding in Patients With Chronic Phase-Chronic Myeloid Leukemia With Complete Hematological Response. Clin Appl Thromb Hemost. 2019; 25: 1076029619858409.

36. Yurttas NO, Eskazan AE. Tyrosine Kinase Inhibitor-Associated Platelet Dysfunction: Does This Need to Have a Significant Clinical Impact? Clin Appl Thromb Hemost. 2019; 25: 1076029619866925.

37. Shoukier M, Borthakur G, Jabbour E, et al. The effect of eltrombopag in managing thrombocytopenia associated with tyrosine kinase therapy in patients with chronic myeloid leukemia and myelofibrosis. Haematologica. 2020; Online ahead of print.

38. Radwi M, Cserti-Gazdewich C. Drug-induced immune thrombocytopenia associated with use of tyrosine kinase inhibitor imatinib. J Taibah Univ Med Sci. 2015; 10: 365-8.

39. Kimbara S, Imamura Y, Yakushijin K, et al. Regorafenib-induced exacerbation of chronic immune thrombocytopenic purpura in remission: A case report. Mol Clin Oncol. 2021; 14: 30.

40. Denorme F, Rondina MT. Targeting Glycoprotein VI for Thromboembolic Disorders. Arterioscler Thromb Vasc Biol. 2019; 39: 839-40.

41. Lockyer S, Okuyama K, Begum S, et al. GPVI-deficient mice lack collagen responses and are protected against experimentally induced pulmonary thromboembolism. Thromb Res. 2006; 118: 371-80.

42. Nieswandt B, Schulte V, Bergmeier W, et al. Long-term antithrombotic protection by in vivo depletion of platelet glycoprotein VI in mice. J Exp Med. 2001; 193: 459-69.

43. Bender M, May F, Lorenz V, et al. Combined in vivo depletion of glycoprotein VI and C-type lectin-like receptor 2 severely compromises hemostasis and abrogates arterial thrombosis in mice. Arterioscler Thromb Vasc Biol. 2013; 33: 926-34.

44. Baaten C, Meacham S, de Witt SM, et al. A synthesis approach of mouse studies to identify genes and proteins in arterial thrombosis and bleeding. Blood. 2018; 132: e35-e46.

45. Nagy M, Perrella G, Dalby A, et al. Flow studies on human GPVI-deficient blood under coagulating and noncoagulating conditions. Blood Adv. 2020; 4: 2953-61.

46. Loren CP, Aslan JE, Rigg RA, et al. The BCR-ABL inhibitor ponatinib inhibits platelet immunoreceptor tyrosine-based activation motif (ITAM) signaling, platelet activation and aggregate formation under shear. Thromb Res. 2015; 135: 155-60.

47. Gratacap MP, Martin V, Valéra MC, et al. The new tyrosine-kinase inhibitor and anticancer drug dasatinib reversibly affects platelet activation in vitro and in vivo. Blood. 2009; 114: 1884-92.

48. Levade M, David E, Garcia C, et al. Ibrutinib treatment affects collagen and von Willebrand factordependent platelet functions. Blood. 2014; 124: 3991-5.

49. Rigg RA, Aslan JE, Healy LD, et al. Oral administration of Bruton's tyrosine kinase inhibitors impairs GPVI-mediated platelet function. Am J Physiol Cell Physiol. 2016; 310: C373-80.

50. De Stefano V. Arterial thrombosis and cancer: the neglected side of the coin of Trousseau syndrome. Haematologica. 2018; 103: 1419-21.

51. Navi BB, Reiner AS, Kamel H, et al. Risk of Arterial Thromboembolism in Patients With Cancer. J Am Coll Cardiol. 2017; 70: 926-38.

52. Tsai TT, Ho PM, Xu S, et al. Increased risk of bleeding in patients on clopidogrel therapy after drugeluting stents implantation: insights from the HMO Research Network-Stent Registry (HMORNstent). Circ Cardiovasc Interv. 2010; 3: 230-5.

53. Pancholia AK. Association of aspirin use with major bleeding in patients with and without diabetes. Indian heart journal. 2012; 64: 615-.

54. Swan D, Loughran N, Makris M, Thachil J. Management of bleeding and procedures in patients on antiplatelet therapy. Blood Rev. 2020; 39: 100619.

55. Hayashi T, Shibata M, Oe S, Miyagawa K, Honma Y, Harada M. Antiplatelet Therapy Improves the Prognosis of Patients with Hepatocellular Carcinoma. Cancers (Basel). 2020; 12.

56. Grancher A, Michel P, Di Fiore F, Sefrioui D. [Aspirin and colorectal cancer]. Bull Cancer. 2018; 105: 171-80.

57. Hu X, Wu LW, Weng X, Lin NM, Zhang C. Synergistic antitumor activity of aspirin and erlotinib: Inhibition of p38 enhanced aspirin plus erlotinib-induced suppression of metastasis and promoted cancer cell apoptosis. Oncol Lett. 2018; 16: 2715-24. 
58. Kanazawa S, Yamaguchi K, Kinoshita Y, et al. Effect of low-dose aspirin for skin rash associated with erlotinib therapy in patients with lung cancer. Platelets. 2009; 20: 70-1.

59. Muktiadmodjo S, Suharti C, Santosa S. Effect of clopidogrel on adenosine diphosphate level during nilotinib therapy in chronic myeloid leukemia. Ann Oncol. 2017; 28: x96.

60. Haouala A, Widmer N, Duchosal MA, Montemurro M, Buclin T, Decosterd LA. Drug interactions with the tyrosine kinase inhibitors imatinib, dasatinib, and nilotinib. Blood. 2011; 117: e75-87.

61. Sabrkhany S, Griffioen AW, Oude Egbrink MG. The role of blood platelets in tumor angiogenesis. Biochim Biophys Acta. 2011; 1815: 189-96.

62. Sabrkhany S, Kuijpers MJE, Oude Egbrink MGA, Griffioen AW. Platelets as messengers of early-stage cancer. Cancer Metastasis Rev. 2021.

63. Cho MS, Bottsford-Miller J, Vasquez HG, et al. Platelets increase the proliferation of ovarian cancer cells. Blood. 2012; 120: 4869-72.

64. Labelle M, Begum S, Hynes RO. Direct signaling between platelets and cancer cells induces an epithelial-mesenchymal-like transition and promotes metastasis. Cancer Cell. 2011; 20: 576-90.

65. Klement GL, Yip TT, Cassiola F, et al. Platelets actively sequester angiogenesis regulators. Blood. 2009; 113: 2835-42.

66. Wojtukiewicz MZ, Sierko E, Hempel D, Tucker SC, Honn KV. Platelets and cancer angiogenesis nexus. Cancer Metastasis Rev. 2017; 36: 249-62.

67. Battinelli EM, Markens BA, Italiano JE, Jr. Release of angiogenesis regulatory proteins from platelet alpha granules: modulation of physiologic and pathologic angiogenesis. Blood. 2011; 118: 1359-69.

68. Yan M, Jurasz P. The role of platelets in the tumor microenvironment: From solid tumors to leukemia. Biochim Biophys Acta. 2016; 1863: 392-400.

69. Huijbers EJ, van Beijnum JR, Thijssen VL, Sabrkhany S, Nowak-Sliwinska P, Griffioen AW. Role of the tumor stroma in resistance to anti-angiogenic therapy. Drug Resist Updat. 2016; 25: 26-37.

70. van Beijnum JR, Nowak-Sliwinska P, Huijbers EJ, Thijssen VL, Griffioen AW. The great escape; the hallmarks of resistance to antiangiogenic therapy. Pharmacol Rev. 2015; 67: 441-61.

71. Iqbal AM, Lopez RA, Hai O. Antiplatelet Medications. StatPearls. Treasure Island (FL): StatPearls Publishing Copyright (C) 2021, StatPearls Publishing LLC., 2021.

72. Rothwell PM, Wilson M, Price JF, Belch JF, Meade TW, Mehta Z. Effect of daily aspirin on risk of cancer metastasis: a study of incident cancers during randomised controlled trials. Lancet. 2012; 379: 1591601.

73. Etulain J, Fondevila C, Negrotto S, Schattner M. Platelet-mediated angiogenesis is independent of VEGF and fully inhibited by aspirin. Br J Pharmacol. 2013; 170: 255-65.

74. Dovizio M, Tacconelli S, Sostres C, Ricciotti E, Patrignani P. Mechanistic and pharmacological issues of aspirin as an anticancer agent. Pharmaceuticals (Basel). 2012; 5: 1346-71.

75. Lin RJ, Afshar-Kharghan V, Schafer Al. Paraneoplastic thrombocytosis: the secrets of tumor selfpromotion. Blood. 2014; 124: 184-7.

76. Sabrkhany S, Kuijpers MJE, Griffioen AW, Oude Egbrink MGA. Platelets: the holy grail in cancer blood biomarker research? Angiogenesis. 2019; 22: 1-2.

77. Sabrkhany S, Griffioen AW, Pineda S, et al. Sunitinib uptake inhibits platelet function in cancer patients. Eur J Cancer. 2016; 66: 47-54.

78. Stevenson JL, Varki A, Borsig L. Heparin attenuates metastasis mainly due to inhibition of P- and L-selectin, but non-anticoagulant heparins can have additional effects. Thromb Res. 2007; 120 Suppl 2: S107-11.

79. Erpenbeck L, Schön MP. Deadly allies: the fatal interplay between platelets and metastasizing cancer cells. Blood. 2010; 115: 3427-36.

80. Coward J, Kulbe H, Chakravarty P, et al. Interleukin- 6 as a therapeutic target in human ovarian cancer. Clin Cancer Res. 2011; 17: 6083-96.

81. Rossi JF, Négrier S, James ND, et al. A phase I/II study of siltuximab (CNTO 328), an anti-interleukin-6 monoclonal antibody, in metastatic renal cell cancer. Br J Cancer. 2010; 103: 1154-62.

82. Hudes G, Tagawa ST, Whang YE, et al. A phase 1 study of a chimeric monoclonal antibody against interleukin-6, siltuximab, combined with docetaxel in patients with metastatic castration-resistant prostate cancer. Invest New Drugs. 2013; 31: 669-76.

83. BOM N-c. Cabozantinib als tweedelijns of laterelijns behandeling van het gevorderd of gemetastaseerd 


\section{Chapter 8}

heldercellig niercelcarcinoom. Med Oncol. 2016: 41-4.

84. BOM N-c. Eerstelijns combinatiebehandeling met pembrolizumab en axitinib bij het gemetastaseerd heldercellig niercelcarcinoom. Med Oncol. 2020: 7-11.

85. Goodman VL, Rock EP, Dagher R, et al. Approval summary: sunitinib for the treatment of imatinib refractory or intolerant gastrointestinal stromal tumors and advanced renal cell carcinoma. Clin Cancer Res. 2007; 13: 1367-73.

86. Numakura K, Fujiyama N, Takahashi M, et al. Clinical implications of pharmacokinetics of sunitinib malate and $\mathrm{N}$-desethyl-sunitinib plasma concentrations for treatment outcome in metastatic renal cell carcinoma patients. Oncotarget. 2018; 9: 25277-84.

87. Hamilton Z, Lee HJ, Jimenez J, et al. Change in platelet count as a prognostic indicator for response to primary tyrosine kinase inhibitor therapy in metastatic renal cell carcinoma. BJU Int. 2016; 118: 927-34.

88. Kim TW, Lee JH, Shim KH, et al. Prognostic significance of preoperative and follow-up neutrophil-tolymphocyte ratio and platelet-to-lymphocyte ratio in patients with non-metastatic clear cell renal cell carcinoma. Investig Clin Urol. 2019; 60: 14-20.

89. Huszno J, Kolosza Z. Prognostic value of the neutrophil-lymphocyte, platelet-lymphocyte and monocyte-lymphocyte ratio in breast cancer patients. Oncol Lett. 2019; 18: 6275-83.

90. Wang HK, Wan FN, Gu WJ, et al. Eosinophil percentage elevation as a prognostic factor for overall survival in patients with metastatic renal cell carcinoma treated with tyrosine kinase inhibitor. Oncotarget. 2016; 7: 68943-53.

91. Verhaart SL, Abu-Ghanem Y, Mulder SF, et al. Real-world Data of Nivolumab for Patients With Advanced Renal Cell Carcinoma in the Netherlands: An Analysis of Toxicity, Efficacy, and Predictive Markers. Clin Genitourin Cancer. 2020.

92. Motzer Criteria. In: Schwab M, ed. Encyclopedia of Cancer. Berlin, Heidelberg: Springer Berlin Heidelberg, 2011, 2377.

93. Ko JJ, Xie W, Kroeger N, et al. The International Metastatic Renal Cell Carcinoma Database Consortium model as a prognostic tool in patients with metastatic renal cell carcinoma previously treated with first-line targeted therapy: a population-based study. Lancet Oncol. 2015; 16: 293-300.

94. Rini BI, Plimack ER, Stus V, et al. Pembrolizumab plus Axitinib versus Sunitinib for Advanced RenalCell Carcinoma. N Engl J Med. 2019; 380: 1116-27.

95. Motzer RJ, Escudier B, McDermott DF, et al. Nivolumab versus Everolimus in Advanced Renal-Cell Carcinoma. N Engl J Med. 2015; 373: 1803-13.

96. Harbi MH, Smith CW, Nicolson PLR, Watson SP, Thomas MR. Novel antiplatelet strategies targeting GPVI, CLEC-2 and tyrosine kinases. Platelets. 2021; 32: 29-41.

97. Payrastre B, Ribes A. Low-dose Btk inhibitors: an 'aspirin' of tomorrow? Haematologica. 2021; 106: 2-4. 

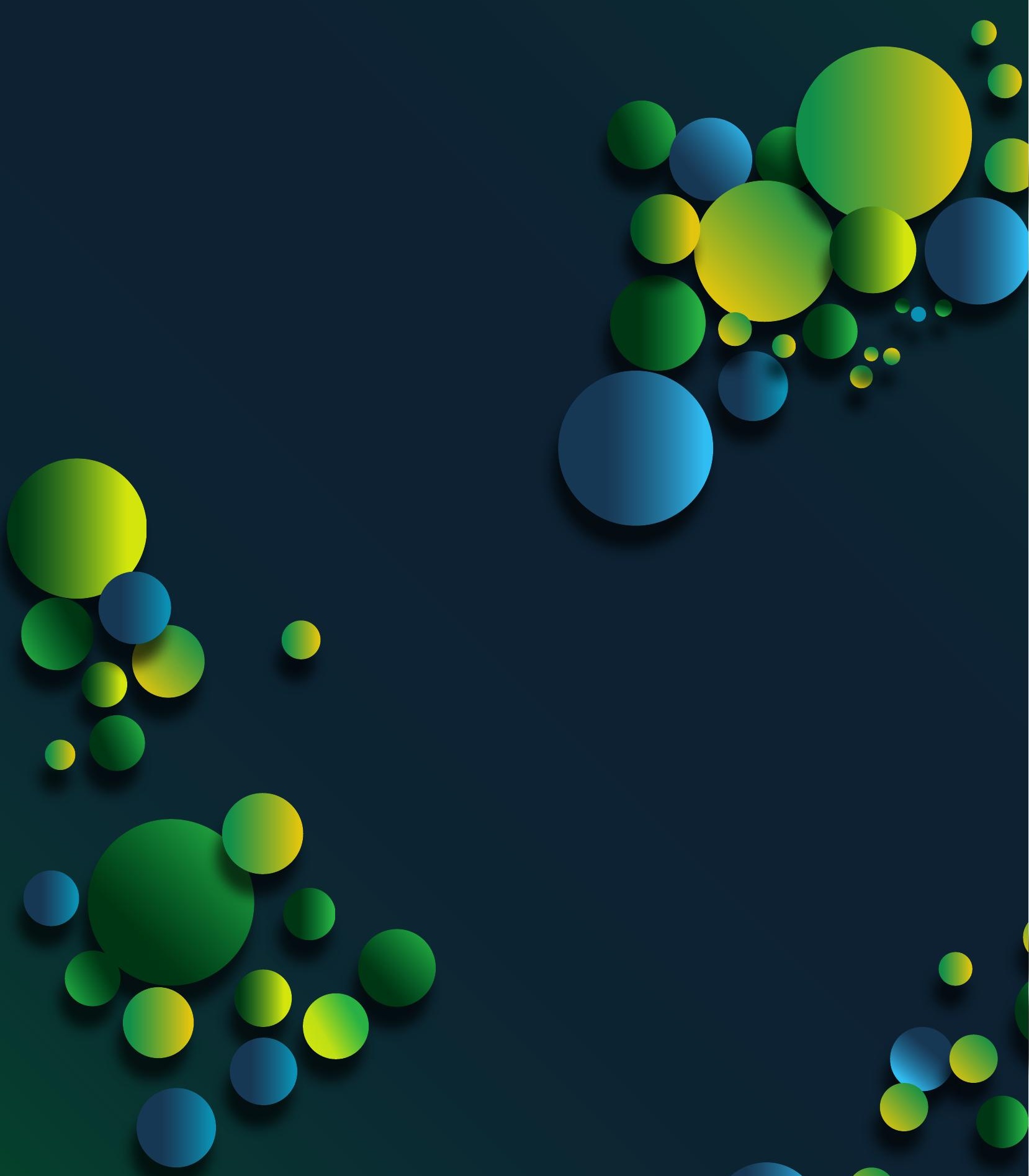


\section{Chapter 9}

Summary

Samenvatting

Impact

Curriculum Vitae

Publications

Acknowledgements 

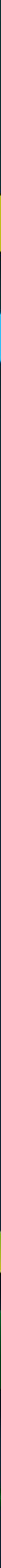


\section{Summary}

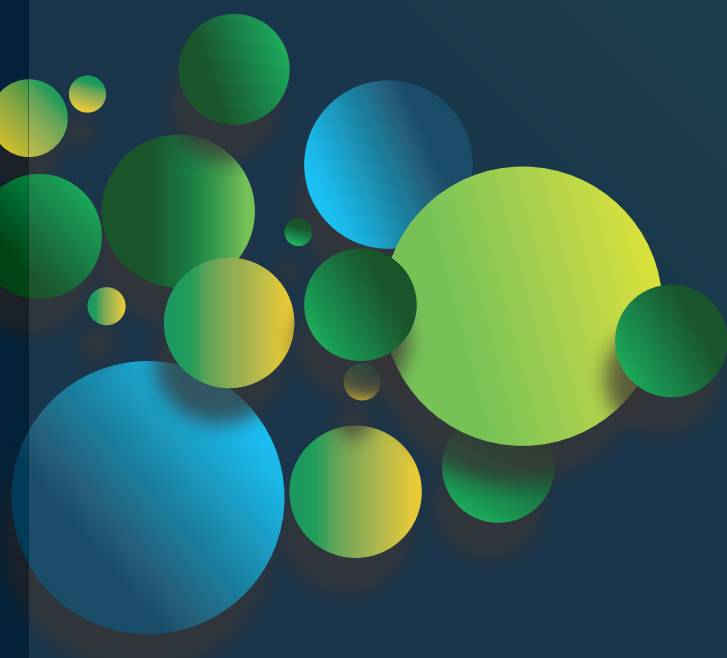




\section{Chapter 9}

In the last decades, tyrosine kinase inhibitors (TKIs) have been developed as a new type of anti-cancer agents. These TKIs inhibit growth factor receptors (tyrosine kinases) involved in angiogenesis and tumour development, thereby improving progression free survival. As tyrosine kinases are expressed in many cell-types, and most TKIs inhibit multiple protein tyrosine kinases, they inevitably have off-target effects. One of the side effects described for several TKIs is (mild) bleeding. Blood platelets, well-known for their involvement in haemostasis, also contain multiple protein tyrosine kinases, which are essential for their activation. Therefore, the overall aim of this thesis was to provide more insights in the unintended effects of TKIs on platelet function.

Chapter 1 provides a brief background on platelet activation processes and thrombus formation, with additional focus on the involvement of tyrosine kinases in the different activation pathways.

To look more closely into the different TKIs and their anti-platelet effects, in Chapter 2 a detailed literature-based review is given, which provides an extended overview and mechanistic insight into the action of TKIs in current clinical use. The targets of these TKIs were compared with the tyrosine kinases present in platelets and the possibility to be linked to anti-platelet effects. This revealed that (for some TKIs) the knowledge on affinity for their targets does not completely align with published effects on platelets and reported bleeding events. In addition, clinical consequences of platelet inhibition by treatment with TKIs were discussed. Conversely, given the role of platelets in tumour progression, we discussed whether platelet inhibition can be an interesting strategy to inhibit tumour growth and monitor response to therapy.

Pazopanib is an oral TKI used for treatment of advanced renal cell carcinoma (RCC). In Chapter 3, we investigated the effects of pazopanib on platelet function in vitro and in RCC patients on-treatment. We found that pazopanib (dose-dependently) reduced GPVI-induced platelet responses and thrombus formation under flow in blood from healthy volunteers. Pazopanib inhibited tyrosine phosphorylation and intracellular calcium signalling in platelets stimulated via the collagen receptor glycoprotein (GP)VI, subsequently leading to reduced exposure of phosphatidylserine (PS) as a marker for procoagulant activity. In addition, we analysed blood samples from 10 RCC patients before and 14 days after receiving pazopanib as monotherapy. This treatment caused an overall lowering in platelet count, with 3 patients experiencing mild bleeding. The effects of pazopanib on these patients' platelets were mainly confined to a reduction in GPVI-dependent procoagulant activity. Control experiments indicated that higher pazopanib concentrations were required to inhibit GPVI-mediated PS exposure in the presence of plasma. Together, these results indicated that pazopanib suppresses GPVI-induced platelet activation responses in a way partly antagonized by the presence of plasma.

Another multi-target TKI used for the treatment of advanced RCC is sunitinib. This compound was reported to affect collagen-induced activation under non-coagulating conditions. In Chapter 4, we investigated the effects of sunitinib on thrombus formation induced by other tyrosine kinase-dependent receptors, as well as the effects under coagulating conditions. We 
observed that both thrombus formation and PS exposure under flow were affected by sunitinib on collagen type I and III. Upon tissue factor-triggered coagulation, sunitinib decreased PS exposure and fibrin formation. In blood from cancer patients more pronounced effects of sunitinib were observed in lung and pancreatic as compared to neuroglioblastoma and other cancer types. Cancer patients commonly suffer from cardiovascular disease as a co-morbidity, and may be at risk for (secondary) thrombotic events. Therefore, these patients are treated with anti-platelet drugs such as aspirin, which irreversibly inhibits platelet activation and is associated with an increased bleeding risk. As both sunitinib and aspirin are associated with bleeding, we further investigated the synergistic effects of both compounds in this setting. Compared to sunitinib alone, the combination of sunitinib with aspirin resulted in further reduction of platelet aggregation, thrombus formation and PS exposure on collagen under flow with(out) coagulation. In conclusion, sunitinib suppressed collagen-induced procoagulant activity and delayed fibrin formation, which was aggravated by aspirin treatment.

To investigate the effects of sunitinib not only in vitro, but also in cancer patients, in Chapter 5 we investigated the quantitative and qualitative changes in platelet traits as a function of the sunitinib levels and the occurrence of bleeding in RCC patients on treatment with sunitinib. Blood was collected from 20 metastatic RCC (mRCC) patients before treatment, and at 2 weeks, 4 weeks and 3 months after sunitinib administration. In sunitinib-treated mRCC patients, concentrations of sunitinib and its active metabolite in plasma and serum were highly correlated. The active metabolite was relatively increased in the patients' platelets compared to sunitinib. On average, a continued reduction in platelet count was observed on-treatment, which was significantly related to the inhibitor levels in plasma/serum. Principal component and correlational analysis showed that the concentration of (active) sunitinib in plasma/serum was linked with a reduction in both platelet count and collagen-induced platelet aggregation. The reduced aggregation in part associated with reported bleeding, but did not correlate to disease progression. In conclusion, sunitinib induced reduction in quantitative and qualitative platelet traits may reflect the effective sunitinib levels in the patient.

The mechanism underlying the increased bleeding tendency with TKIs often remains unclear. Therefore, in Chapter $\mathbf{6}$ we investigated the effects of three Btk inhibitors, two irreversible (ibrutinib and acalabrutinib) and one novel reversible inhibitor (MK-1026), on platelet function in healthy volunteers, patients and Btk deficient mice, together with off-target effects on tyrosine kinase phosphorylation. All three inhibitors impaired GPVI- and CLEC-2-mediated aggregation, activation and secretion in isolated platelets in a dose-dependent manner. Only ibrutinib inhibited thrombus formation on von Willebrand Factor (vWF) co-coated surfaces, while on collagen this process was not affected. In blood from Btk-deficient mice, collagen-induced thrombus formation under flow was reduced, but preincubation with either inhibitor was without additional effects. MK-1026 showed less off-target effects upon GPVI-induced tyrosine kinase phosphorylation as 


\section{Chapter 9}

compared to ibrutinib and acalabrutinib. In ibrutinib-treated patients, GPVI-stimulated platelet activation and adhesion on VWF-co-coated surfaces were inhibited, while CLEC-2 stimulation induced variable responses. The dual inhibition of GPVI and CLEC-2 signalling by Btk inhibitors might account for the increased bleeding tendency, with ibrutinib causing more high-grade bleedings due to additional inhibition of platelet-vWF interaction. As MK-1026 showed less offtarget effects and only affected activation of isolated platelets, it might be promising for future treatment.

Although multiple TKIs are reported to increase the risk of bleeding, mainly minor bleeding symptoms are described. The currently used anti-platelet drugs for treatment of arterial thrombosis coincide with a greater risk of bleeding. Hence, platelet inhibitors that preserve haemostasis are warranted. In Chapter 7, we investigated the anti-platelet properties of several clinically used TKIs as exploration of possible repurposing of these compounds as anti-platelet drugs. Eight TKIs were selected based on the affinity for tyrosine kinases expressed in platelets and published bleeding symptoms. Overall, the TKIs with the highest affinities for platelet targets most strongly inhibited platelet function. Dasatinib and sunitinib dose-dependently reduced collageninduced aggregation in platelet-rich plasma and washed platelets, while pazopanib, cabozantinib and vatalanib inhibited this response in washed platelets only, and fostamatinib, axitinib and lapatinib showed no or limited effects. Fostamatinib effectively reduced whole blood thrombus formation on collagen under flow, as well as on vWF plus rhodocytin or laminin. Pazopanib, sunitinib, dasatinib, axitinib and vatalanib (mildly) reduced thrombus formation only on collagen. Intracellular calcium measurements and activation responses in isolated platelets were inhibited by dasatinib, fostamatinib, sunitinib and pazopanib. Simultaneous measurement of different platelet populations raised by collagen receptor-stimulation showed that fostamatinib, cabozantinib and vatalanib decreased highly activated platelet populations, whereas negative, resting populations were increased. Altogether, the inhibitory effects of dasatinib, fostamatinib, sunitinib and pazopanib suggested interference in early collagen receptor-induced signalling events, whereas for cabozantinib and vatalanib the effects may be more downstream. Fostamatinib, sunitinib, pazopanib and vatalanib have been associated with mild or no bleeding events and, hence, may be promising candidates to explore further as potential anti-platelet drugs.

In Chapter $\mathbf{8}$ are the most important findings of this thesis are discussed in perspective of the current literature. With this thesis we would like to raise awareness for the potential antiplatelet effects of several TKIs during cancer treatment, which could be enhanced in the presence of antithrombotic drugs, thereby increasing the bleeding risk. Changes in platelet features and unbound plasma concentrations of TKIs upon treatment could provide tools for monitoring and managing (these) side effects. 
9 

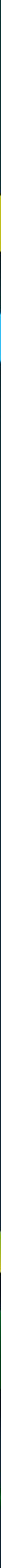


\section{Samenvatting}




\section{Chapter 9}

In de afgelopen decennia zijn tyrosine-kinaseremmers (TKI's) ontwikkeld als een nieuw type antikankerbehandeling. Deze TKI's remmen in de eerste plaats groeifactor-receptoren (tyrosine kinasen) op tumor- en vaatwandcellen. Op deze manier worden angiogenese en tumorontwikkeling geremd, waardoor de progressie-vrije overleving bij patiënten wordt verbeterd. Omdat tyrosine kinasen in veel celtypen in het lichaam tot expressie komen, hebben ze onvermijdelijk bijwerkingen. Een van de beschreven bijwerkingen bij meerdere TKI's is (milde) bloedingen. Bloedplaatjes, die bekend staan om hun betrokkenheid bij hemostase, bevatten ook meerdere tyrosine kinasen die essentieel zijn voor hun activering. Het algemene doel van dit proefschrift was om meer inzicht te geven in de onbedoelde effecten van TKI's op de functie van bloedplaatjes. Hoofdstuk 1 geeft een korte achtergrond over de processen van activering van bloedplaatjes en daaropvolgende trombusvorming, waarin we extra focus leggen op de betrokkenheid van tyrosine kinasen in de verschillende activeringsroutes.

Om meer inzicht te krijgen in de verschillende TKI's en hun effecten op bloedplaatjes effecten, wordt in Hoofdstuk $\mathbf{2}$ een gedetailleerd literatuuronderzoek beschreven. Hierin tonen we een uitgebreid overzicht van TKI's die in de huidige kliniek gebruikt worden voor de behandeling van verschillende soorten kanker, almede de cellulaire aangrijpingspunten voor hun werking. De doelwitten van deze TKI's zijn vergeleken met de tyrosine kinasen die aanwezig zijn in bloedplaatjes, en indien mogelijk gekoppeld aan anti-bloedplaatjes effecten. Hieruit bleek dat (voor sommige TKI's) de kennis over affiniteit voor hun doelwitten niet volledig overeenkomt met gepubliceerde effecten op bloedplaatjes en de gerapporteerde bloedingen. Daarnaast zijn de klinische gevolgen van bloedplaatjesremming door behandeling met TKI's besproken. Omgekeerd, gezien de rol van bloedplaatjes in tumorprogressie, vroegen we ons af of bloedplaatjesremming een interessante strategie zou kunnen zijn om tumorgroei te remmen, danwel de respons op therapie te volgen.

Pazopanib is een orale TKI die gebruikt wordt voor de behandeling van gevorderd niercelcarcinoom (RCC). In Hoofdstuk 3 onderzochten we de effecten van pazopanib op bloedplaatjesfunctie in vitro en bij RCC-patiënten tijdens hun behandeling. De resultaten lieten zien dat pazopanib (dosisafhankelijk) de bloedplaatjesreacties en trombusvorming onder stromingscondities na stimulatie van de collageen receptor glycoproteïne VI (GPVI) verminderde in het bloed van gezonde vrijwilligers. Pazopanib remde de fosforylering van tyrosine kinasen en de intracellulaire calciumsignalering in bloedplaatjes gestimuleerd via GPVI. Dit leidde vervolgens tot verminderde expressie van fosfatidylserine (PS) welke belangrijk is voor het bevorderen van de bloedstolling, de zogenaamde procoagulante activiteit. Daarnaast analyseerden we bloedmonsters van 10 RCC-patiënten vóór en 14 dagen na inname van pazopanib als monotherapie. Deze behandeling veroorzaakte een algehele verlaging van het aantal bloedplaatjes, waarbij 3 patiënten lichte bloedingen ervaarden. De effecten van pazopanib op de bloedplaatjes van deze patiënten waren voornamelijk beperkt tot een vermindering van 
de GPVI-afhankelijke bevordering van de procoagulante activiteit. Controle experimenten lieten zien dat hogere pazopanib concentraties nodig waren om de GPVI-gemedieerde PS-expressie in aanwezigheid van plasma te kunnen remmen. Samen gaven deze resultaten aan dat pazopanib de GPVI-geïnduceerde activeringsreacties van bloedplaatjes onderdrukt op een manier die gedeeltelijk wordt tegengewerkt door de aanwezigheid van plasma.

Een andere TKI die wordt gebruikt voor de behandeling van gevorderd RCC is sunitinib. Van dit geneesmiddel is beschreven dat deze de collageen-geïnduceerde activering van bloedplaatjes onder niet-stollende omstandigheden beïnvloedt. In Hoofdstuk 4 onderzochten we de effecten van sunitinib op trombusvorming geïnduceerd door andere tyrosine kinase-afhankelijke receptoren, evenals de effecten onder stollende condities. We hebben waargenomen dat zowel trombusvorming als PS-expressie onder stromingscondities werden geremd door sunitinib op collageen type I en III. Bij aanwezigheid van weefselfactor om bloedstolling te induceren, verminderde sunitinib de PS-expressie op bloedplaatjes en de vorming van fibrine. In bloed van kankerpatiënten werden meer uitgesproken effecten van sunitinib waargenomen in longen alvleesklierkanker in vergelijking tot hersenkanker (neuroglioblastoma) en andere typen van kanker. Kankerpatiënten lijden vaak mede aan hart- en vaatziekten en hebben mogelijk verhoogd risico op (secundaire) trombotische gebeurtenissen. Daarom worden deze patiënten behandeld met medicatie tegen bloedplaatjesaggregatie zoals aspirine, wat de activering van bloedplaatjes onomkeerbaar remt en gepaard gaat met een verhoogd bloedingsrisico. Omdat zowel sunitinib als aspirine geassocieerd zijn met een verhoogd risico op bloedingen, hebben we de synergistische effecten van beide geneesmiddelen in deze setting verder onderzocht. Vergeleken met sunitinib alleen, resulteerde de combinatie van sunitinib en aspirine in een verdere vermindering van de aggregatie, trombusvorming en PS-expressie van bloedplaatjes op het collageen oppervlak onder stromingscondities, zowel in aan- als afwezigheid van bloedstolling. Samenvattend, sunitinib onderdrukte de collageen-geïnduceerde bevordering van de bloedstolling en vertraagde de vorming van fibrine; deze onderdrukking werd versterkt door behandeling met aspirine.

In Hoofdstuk $\mathbf{5}$ hebben we de kwantitatieve en kwalitatieve veranderingen in eigenschappen van bloedplaatjes onderzocht in relatie tot de sunitinib concentratie in het bloed, en het optreden van bloedingen bij RCC-patiënten na behandeling met sunitinib. Bloed werd afgenomen van 20 patiënten met gevorderd RCC vóór start met behandeling en 2 weken, 4 weken en 3 maanden na behandeling met sunitinib. De concentraties van sunitinib en de actieve metaboliet in het plasma en serum van RCC-patiënten behandeld met sunitinib waren sterk gecorreleerd. De concentratie van de actieve metaboliet was relatief verhoogd in de bloedplaatjes van de patiënten in vergelijking met sunitinib. Gemiddeld werd er tijdens de behandeling een aanhoudende afname van het aantal bloedplaatjes waargenomen, wat significant verband hield met de concentratie van de remmers in het plasma en/of serum. Principal component- en correlatieanalyses toonden aan dat de concentratie van (actief) sunitinib in het plasma en/of 


\section{Chapter 9}

serum verband hield met een vermindering van zowel het aantal bloedplaatjes als de collageengeïnduceerde aggregatie. De verminderde aggregatie was gedeeltelijk geassocieerd met de gerapporteerde bloedingen, maar correleerde niet met de progressie van de ziekte. Concluderend kan de door sunitinib geïnduceerde vermindering van kwantitatieve en kwalitatieve kenmerken van bloedplaatjes een weerspiegeling zijn van de effectieve sunitinib concentratie in de patiënt.

Het mechanisme dat ten grondslag ligt aan de verhoogde bloedingsneiging bij TKI's blijft vaak onduidelijk. Daarom hebben we in Hoofdstuk 6 de (onbedoelde) effecten onderzocht van drie Btk-remmers op de functie van bloedplaatjes in het bloed van gezonde vrijwilligers, patiënten en Btk-deficiënte muizen. Het betreft twee irreversibele remmers (ibrutinib en acalabrutinib), en een nieuwe, reversibele remmer (MK-1026). Alle drie de remmers verminderden de GPVIen CLEC-2-gemedieerde aggregatie, activatie en secretie van geïsoleerde bloedplaatjes op een dosisafhankelijke manier. Alleen ibrutinib verminderde trombusvorming op von Willebrand Factor (VWF) in combinatie met rhodocytine, laminine of fibrinogeen, terwijl op collageen geen effecten werden gezien. In het bloed van Btk-deficiënte muizen was de collageen-geïnduceerde trombusvorming onder stromingscondities verminderd, maar er werden geen additionele effecten waargenomen na toevoeging van de Btk-remmers. MK-1026 liet minder onbedoelde bijwerkingen zien bij GPVI-gestimuleerde tyrosine kinase fosforylatie in vergelijking tot ibrutinib en acalabrutinib. Bij patiënten onder behandeling met ibrutinib werden de GPVI-geïnduceerde activering en adhesie van bloedplaatjes geremd op de oppervlakken gecombineerd met vWF, terwijl variabele responsen werden waargenomen met CLEC-2 stimulatie. De duale remming van GPVI- en CLEC-2-signalering door Btk-remmers kan verantwoordelijk zijn voor de verhoogde bloedingsneiging, met meer hoogwaardige bloedingen bij behandeling met ibrutinib als gevolg van extra remming van bloedplaatjes adhesie aan vWF. Omdat MK-1026 minder off-target effecten liet zien en alleen de activatie van geïsoleerde bloedplaatjes beïnvloedde, zou dit een veelbelovende toekomstige behandeling kunnen zijn.

Hoewel het is beschreven dat meerdere TKI's het risico op bloedingen verhogen, betreft dit voornamelijk milde bloedingssymptomen. De momenteel voorgeschreven medicatie tegen aggregatie van bloedplaatjes ter behandeling van arteriële trombose geven een groter risico op bloedingen. Daarom zijn bloedplaatjes remmers noodzakelijk die de hemostase behouden.

In Hoofdstuk 7 hebben we van verschillende klinisch gebruikte TKI's de remmende effecten op bloedplaatjes onderzocht ter verkenning van het gebruik van deze geneesmiddelen als antibloedplaatjes medicatie. Acht TKI's werden geselecteerd op basis van de affiniteit voor tyrosine kinasen die tot expressie komen in bloedplaatjes en de gepubliceerde bloedingssymptomen. Over het algemeen remden de TKI's met de hoogste affiniteit voor tyrosine kinasen in bloedplaatjes, de functie van bloedplaatjes het sterkst. Dasatinib en sunitinib verminderden dosisafhankelijk de collageen-geïnduceerde aggregatie in zowel de aanwezigheid van plasma, als in geïsoleerde bloedplaatjes. Terwijl pazopanib, cabozantinib en vatalanib deze respons alleen in geïsoleerde 
bloedplaatjes remden. Fostamatinib, axitinib en lapatinib vertoonden geen of beperkte effecten. Fostamatinib verminderde effectief de vorming van een trombus in bloed dat werd gestroomd over een collageen oppervlak, evenals over een oppervlak van vWF samen met rhodocytine of laminine. Pazopanib, sunitinib, dasatinib, axitinib en valatalanib verminderden (licht) de vorming van de trombus alleen op het collageen oppervlak. Intracellulaire calciummetingen en activeringsreacties in geïsoleerde bloedplaatjes werden geremd door dasatinib, fostamatinib, sunitinib en pazopanib. Gelijktijdige meting van verschillende populaties van bloedplaatjes opgewekt door stimulatie van de collageen receptor toonde aan dat fostamatinib, cabozantinib en vatalanib de sterk geactiveerde populatie van bloedplaatjes verlaagden, terwijl de negatieve, rustende populaties toenamen. Al met al suggereerden de remmende effecten van dasatinib, fostamatinib, sunitinib en pazopanib interferentie in de vroege signalering van de collageen receptor-geïnduceerde activering, terwijl voor cabozantinib en vatalanib de effecten verder stroomafwaarts kunnen zijn. Fostamatinib, sunitinib, pazopanib en vatalanib zijn in verband gebracht met milde of geen bloedingen en kunnen daarom veelbelovende kandidaten zijn om verder te onderzoeken als potentiële geneesmiddelen om bloedplaatjes te remmen.

In Hoofdstuk 8 worden de belangrijkste bevindingen van dit proefschrift besproken in het perspectief van de huidige literatuur. Met dit proefschrift willen we het bewustzijn vergroten voor de mogelijke remmende effecten van verschillende TKI's op bloedplaatjes tijdens de behandeling van kanker. Deze zouden bovendien versterkt kunnen worden in aanwezigheid van antitrombotische middelen, waardoor het risico op bloedingen verder toeneemt. Veranderingen in de kenmerken van bloedplaatjes en ongebonden plasmaconcentraties na behandeling met TKI's kunnen handvaten bieden voor het monitoren en beheersen van (deze) bijwerkingen. 

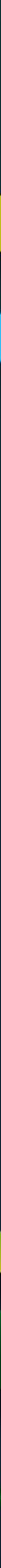


\section{Impact}




\section{Chapter 9}

Cancer is a leading cause of death worldwide, with almost 10 million deaths in $2020^{1}$. One of the hallmarks of cancer is to chronically sustain cell proliferation caused by mutations in or amplification of oncogene-encoded proteins or growth factor receptors ${ }^{2}$. As these proteins include (tyrosine) kinases, cancer cells are described to have abnormal kinase activity which is known to drive processes such as cell proliferation, differentiation, migration, survival and angiogenesis ${ }^{3,4}$. In the past two decades, increased understanding of this process has resulted in the development of many small molecule tyrosine kinase inhibitors (TKIs) as targeted therapy for cancer. In current settings of cancer treatment, TKIs significantly increase the progression-free survival of patients upon (lifelong) treatment. As tyrosine kinases are present in many cell-types, long-term treatment is often associated with (serious) adverse events including bleeding.

Platelets are important players in haemostasis and platelet production and function rely on several tyrosine kinases, suggesting that platelet count and function may be affected by TKIs. Hence, this may play a role in the increased risk of bleeding observed upon treatment with several TKIs. Yet, knowledge on whether and/or how platelets are affected by TKIs is limited. Therefore, we aimed to provide more insights in the effects of different TKIs on platelet function. To define what was known on the relation between TKIs and platelets at the start of this research, in Chapter $\mathbf{2}$ an overview is given of the currently used TKIs in cancer treatment and the effects on platelet function. In order to extent the existing knowledge, we investigated platelet responses in the presence of different TKIs in vitro and in cancer patients (Chapter 3-7). Throughout this thesis, we showed that the majority of TKIs inhibit platelet responses mediated by the collagen receptor glycoprotein (GP)VI, e.g. activation, aggregation and/or thrombus formation. In some cases (additionally) CLEC-2- or GPIb-induced thrombus formation was inhibited by TKI treatment. These results unravelled parts of the underlying mechanism of platelet inhibition by these TKIs. As these mechanisms still remain incomplete or unknown for some TKIs, this could provide new fields of interest for fellow researchers. Furthermore, with our studies involving (specific) cancer patients upon TKI treatment (Chapter 3, $\mathbf{5}$ and $\mathbf{6}$ ), we were able to gain knowledge on the interactions between platelet traits and TKI levels in the blood of these patients. We found that in renal cell carcinoma patients not only platelet function, but also platelet count, was affected by the TKIs sunitinib and pazopanib. For sunitinib, we discovered that the (active) compound levels in plasma or serum correlated with platelet count and function in these patients. This effect was measured already two weeks after the start of treatment, while response to therapy is normally not monitored until three months after start of treatment by a CT-scan. These plasma concentrations of sunitinib (or its metabolite) could be of importance for toxicity and the response to treatment, pointing towards platelets as a monitoring tool for compound effects, both on- and off-target. These results could be of interest for clinicians and cancer patients (hopefully) resulting in (early) therapeutic drug monitoring and increased quality of life during treatment. Therefore, more studies on the unintended side effects/off-target effects of drugs (not only on platelets, but 
also other cells) could be of great importance to provide ways to monitor and improve treatment. These studies would enhance our understanding of the exact mechanism underlying the side effects, which could lead to the development of new drugs with reduced side effects.

Cancer patients often have a cardiovascular history ${ }^{5,6}$ and are prescribed with anti-platelet or anti-coagulant drugs to prevent recurrent cardiovascular events. As anti-platelet medications, such as clopidogrel and aspirin, are also associated with an increased bleeding risk bleeding ${ }^{7-9}$, in Chapter 4 we examined whether there are synergistic effects on platelet function of dual treatment that could further increase the bleeding risk in TKI-treated cancer patients. We showed that aspirin aggravated the effects of sunitinib in platelet aggregation as well as delaying plateletdependent coagulation in whole blood perfusion over a collagen surface. With these results we would like to raise awareness among clinicians of combining anti-platelet therapies and TKIs as this may result in an increased bleeding risk in patients.

As current anti-platelet therapies are associated with bleeding and thrombotic events may still occur, there is a need of better anti-thrombotic drugs. The platelet GPVI and CLEC-2 receptors are important players in arterial thrombosis and thrombo-inflammation with supporting roles in haemostasis ${ }^{10}$. A deficiency of GPVI or CLEC-2 does not significantly influence haemostasis ${ }^{11,}$ ${ }^{12}$, making these receptors interesting targets for anti-thrombotic drugs ${ }^{10,13}$. As these receptors rely on tyrosine kinases for their underlying signalling pathways, we studied the anti-platelet properties of several clinically used TKIs with the aim to explore possible repurposing of these compounds as anti-platelet drugs. As several of the tested TKIs are described with only minor bleeding events, the GPVI inhibition observed with these TKIs might suggest that these could be promising candidates as anti-platelet drugs. However, more research with regard to dosage to estimate efficacy and toxicity in thrombotic patients is needed. With additional research, repurposing TKIs could be of great interest for clinicians, in particular cardiologists, as these TKIs are well-tolerated and already orally available in the clinic. 


\section{References}

1. Ferlay J, Ervik M, Lam F, et al. Global cancer observatory: cancer today. Lyon: international agency for research on cancer. 2020.

2. Hanahan D, Weinberg RA. Hallmarks of cancer: the next generation. Cell. 2011; 144: 646-74.

3. Gross S, Rahal R, Stransky N, Lengauer C, Hoeflich KP. Targeting cancer with kinase inhibitors. J Clin Invest. 2015; 125: 1780-9.

4. Paul MK, Mukhopadhyay AK. Tyrosine kinase- Role and significance in cancer. Int J Med Sci. 2004; 1: $101-15$

5. De Stefano V. Arterial thrombosis and cancer: the neglected side of the coin of Trousseau syndrome. Haematologica. 2018; 103: 1419-21.

6. Navi BB, Reiner AS, Kamel H, et al. Risk of arterial thromboembolism in patients with cancer. J Am Coll Cardiol. 2017; 70: 926-38.

7. Tsai TT, Ho PM, Xu S, et al. Increased risk of bleeding in patients on clopidogrel therapy after drugeluting stents implantation: insights from the HMO Research Network-Stent Registry (HMORN-stent). Circ Cardiovasc Interv. 2010; 3: 230-5.

8. Pancholia AK. Association of aspirin use with major bleeding in patients with and without diabetes. Indian heart journal. 2012; 64: 615-.

9. Swan D, Loughran N, Makris M, Thachil J. Management of bleeding and procedures in patients on antiplatelet therapy. Blood Rev. 2020; 39: 100619.

10. Harbi MH, Smith CW, Nicolson PLR, Watson SP, Thomas MR. Novel antiplatelet strategies targeting GPVI, CLEC-2 and tyrosine kinases. Platelets. 2021; 32: 29-41.

11. Nagy M, Perrella G, Dalby A, et al. Flow studies on human GPVI-deficient blood under coagulating and noncoagulating conditions. Blood Adv. 2020; 4: 2953-61.

12. May F, Hagedorn I, Pleines I, et al. CLEC-2 is an essential platelet-activating receptor in hemostasis and thrombosis. Blood. 2009; 114: 3464-72.

13. Payrastre B, Ribes A. Low-dose Btk inhibitors: an 'aspirin' of tomorrow? Haematologica. 2021; 106: $2-4$. 
9 

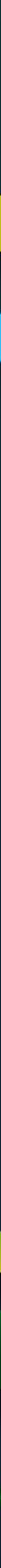


\section{Curriculum Vitae}




\section{Chapter 9}

Beatrice Tullemans, roepnaam Bibian, werd geboren op 27 oktober 1992 te Weert, Nederland. In 2005 begon ze haar middelbare schoolopleiding aan de Philips van Horne SG te Weert, waar ze het HAVO diploma behaalde in 2010 (profiel Natuur \& Gezondheid en Natuur \& Techniek). Vervolgens startte ze een bacheloropleiding Applied Sciences, in de richting Life Sciences, aan de Fontys Hogeschool te Eindhoven. Na het behalen van het bachelorsdiploma in 2014, volgde ze de masteropleiding Biomedical Sciences aan de Universiteit van Maastricht, welke ze met succes afrondde in 2016. Tijdens deze opleidingen liep zij onderzoekstages met als onderwerp immuniteit bij koeien (Vakgroep Infectieziekten en Immunolgie, Universiteit Utrecht), ontwikkeling van immuniteit bij de mens (Vakgroep Interne Geneeskunde, Universiteit Maastricht), invloed van macrofagen op de atherosclerose (Vakgroep Pathologie, Universiteit Maastricht) en de invloed van kankerbehandelingen op de hemostase (Vakgroep Biochemie, Universiteit Maastricht). In november 2016 begon zij als PhD-student in het Onderzoeksschool Hart- en Vaatziekten Maastricht (CARIM) aan de Universiteit Maastricht binnen de Vakgroep Biochemie. Hier heeft ze wetenschappelijk onderzoek uitgevoerd op het gebied van bloedplaatjes, hemostase en kankerbehandeling, zoals in dit proefschrift beschreven. Zij werd hierin begeleid door Dr. Marijke J.E. Kuijpers, Dr. Maureen J.B. Aarts en Prof. Dr. Johan W.M. Heemskerk. Tijdens haar promotie-onderzoek voerde zij projecten uit in de laboratoria van Prof. Dr. C. Oury (Liège, België), Prof. Dr. A. Greinacher (Greifswald, Duitsland), Prof. Dr. W. Ouwehand (Cambridge, Verenigd Koninkrijk) en Prof. Dr. P. Simioni (Padua, Italië). Haar promotieonderzoek werd gepresenteerd op verschillende (inter)nationale congressen. 
Beatrice Tullemans, called Bibian, was born on 27 th of October 1992 in Weert, the Netherlands. In 2005 she started her secondary school at the Philips van Horne SG in Weert, where she obtained her HAVO diploma in 2010 (specialization Nature \& Health and Nature \& Technics). In the same year she started a bachelor study in Applied Sciences, with Life Sciences as specialization, at the Fontys University in Eindhoven. After graduating for her bachelor's degree in 2014, she continued education with a master in Biomedical Sciences at Maastricht University, where she obtained her degree in 2016. During this education, she performed internships on immunity in cows (Department of Infectious Diseases and Immunology, Utrecht University), development of immune cells in humans (Department of Internal Medicine, Maastricht University), effects of macrophages in atherosclerosis (Department of Pathology, Maastricht University) and effects of anti-cancer drugs on platelets in haemostasis (Department of Biochemistry, Maastricht University). In November 2016, she started as a PhD-student in the Cardiovascular Research Institute Maastricht (CARIM) at the Department of Biochemistry of Maastricht University. As a PhD-student, she performed research on platelets, hemostasis and anti-cancer drugs, such as described in this thesis. She performed this research under the supervision of Dr. Marijke J.E. Kuijpers, Dr. Maureen J.B. Aarts and Prof. Dr. Johan W.M. Heemskerk. As a PhD student, she worked in the laboratories of Prof. Dr. C. Oury (Liège, Belgium), Prof. Dr. A. Greinacher (Greifswald, Germany), Prof. Dr. W. Ouwehand (Cambridge, United Kingdom) and Prof. Dr. P. Simioni (Padova, Italy). She presented her research at several (inter)national scientific conferences. 

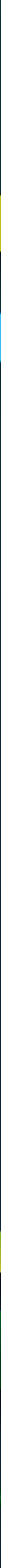


\section{Publications}




\section{Full papers}

1. Marsch E, Demandt JA, Theelen TL, Tullemans BME, Wouters K, Boon MR, van Dijk TH, Gijbels MJ, Dubois LJ, Meex SJ, Mazzone M, Hung G, Fisher EA, Biessen EA, Daemen MJ, Rensen PC, Carmeliet P, Groen AK, Sluimer JC. Deficiency of the oxygen sensor prolyl hydroxylase 1 attenuates hypercholesterolaemia, atherosclerosis, and hyperglycaemia. Eur Heart J 2016;37(39):2993-2997.

2. Mori J, Nagy Z, Di Nunzio G, Smith CW, Geer MJ, Al Ghaithi R, van Geffen JP, Heising S, Boothman L, Tullemans BME, Correia JN, Tee L, Kuijpers MJE, Harrison P, Heemskerk JWM, Jarvis GE, Tarakhovsky A, Weiss A, Mazharian A, Senis YA. Maintenance of murine platelet homeostasis by the kinase Csk and phosphatase CD148. Blood 2018; 131(10):1122-1144.

3. Geer MJ, van Geffen JP, Gopalasingam P, Vögtle T, Smith CW, Heising S, Kuijpers MJE, Tullemans BME, Jarvis GE, Eble JA, Jeeves M, Overduin M, Heemskerk JWM, Mazharian A, Senis YA. Uncoupling ITIM receptor G6b-B from tyrosine phosphatases Shp1 and Shp2 disrupts murine platelet homeostasis. Blood 2018; 132(13):1413-1425.

4. Tullemans BME, Heemskerk JWM, Kuijpers MJE. Acquired platelet antagonism: off-target antiplatelet effects of malignancy treatment with tyrosine kinase inhibitors. $\boldsymbol{J}$ Thromb Haemost 2018; 16(9):1686-1699.

5. Tullemans BME, Nagy M, Sabrkhany S, Griffioen AW, oude Egbrink MGA, Aarts M, Heemskerk JWM, Kuijpers MJE. Tyrosine Kinase Inhibitor Pazopanib Inhibits Platelet Procoagulant Activity in Renal Cell Carcinoma Patients. Front Cardiovasc Med 2018; 5:142.

6. Sternkopf M, Nagy M, Baaten CCFMJ, Kuijpers MJE, Tullemans BME, Wirth J, Theelen W, Mastenbroek TG, Lehrke M, Winnerling B, Baerts L, Marx N, de Meester I, Döring Y, Cosemans JMEM, Daiber A, Steven S, Jankowski J, Heemskerk JWM, Noels H. Native glucagon-like peptide-1 but not its DPP4-cleaved form is a natural suppressor of thrombus growth under physiological flow conditions. Atheroscler Thromb Vasc Biol 2020; 40(3):e65-e77

7. Van Geffen JP, Swieringa F, van Kuijk K, Tullemans BME, Solari FA, Peng B, Clemetson KJ, Farndale RW, Dubois LJ, Sickmann A, Zahedi RP, Ahrends R, Biessen EAL, Sluimer JC, Heemskerk JWM, Kuijpers MJE. Mild hyperlipidemia in mice aggravates platelet responsiveness in thrombus formation and exploration of platelet proteome and lipidome. Sci Rep 2020; 10(1):21407

8. Dickhout A*, Tullemans BME*, Heemskerk JWM, Thijssen VLJL, Kuijpers MJE, Koenen RR. Galectin-1 and platelet factor 4 (CXCL4) induce complementary responses in washed platelets. PLoS One 2021; 16(1):e0244736; *equal contribution

9. Tullemans BME, Fernandez DI*, Veninga A*, Baaten CCFMJ, Peters LJF, Aarts MJB, Eble JA, Campello E, Spiezia L, Simioni P, van der Vorst EPC, van der Meijden PEJ, Heemskerk JWM, Kuijpers MJE. Tyrosine kinase inhibitor sunitinib delays platelet-induced coagulation: additive effects of aspirin. Thromb Haemost 2021; Online ahead of print. 
10. Tullemans BME, Karel MFA, Léopold V, ten Brink SM, Baaten CCFMJ, Maas SL, de Vos AF, Eble JA, Nijziel MR, van der Vorst EPC, Cosemans JMEM, Heemskerk JWM, Claushuis TAM, Kuijpers MJE. Comparison of inhibitory effects of irreversible and reversible Btk inhibitors on platelet function. Br J Haematol 2021; 1-15.

11. Veninga A, Baaten CCFMJ, Tullemans BME, de Simone I, Kuijpers MJE, Heemskerk JWM, van der Meijden PEJ. Effects of platelet agonists and priming on formation of platelet populations.

Thromb Haemost 2021; Online ahead of print.

\section{Abstracts}

1. Tullemans BME, Nagy M, Sabrkhany S, Aarts M, Heemskerk JWM and Kuijpers MJE. Tyrosine kinase inhibitor pazopanib inhibits platelets procoagulant activity in vitro and in cancer patients. Congress of the International Society on Thrombosis and Haemostasis (ISTH), Berlin, Germany, 2017.

2. Tullemans BME, Nagy M, Sabrkhany S, Griffioen AW, oude Egbrink MGA, Aarts MJB, Heemskerk JWM and Kuijpers MJE. Platelet procoagulant activity is inhibited by the tyrosine kinase inhibitor pazopanib in vitro and in renal cell carcinoma patients. Centre for Thrombosis and Haemostasis (CTH) retreat, Mainz, Germany, 2017.

3. Tullemans BME, Nagy M, Sabrkhany S, Griffioen AW, oude Egbrink MGA, Aarts MJ, Heemskerk JWM and Kuijpers MJE. Platelet procoagulant activity is inhibited by tyrosine kinase inhibitor pazopanib in vitro and in renal cell carcinoma patients. $1^{\text {st }}$ Italian-UK platelet meeting, Bath, United Kingdom, 2017.

4. Tullemans BME, Nagy M, Sabrkhany S, Griffioen AW, oude Egbrink MGA, Aarts MJ, Heemskerk JWM and Kuijpers MJE. Anti-platelet effects of tyrosine kinase inhibitors used for cancer treatment: pazopanib as a proof of principle. $4^{\text {th }}$ European Platelet Network Conference (EUPLAN), Bruges, Belgium, 2018.

5. Van Geffen JP, van Kuijk K, Tullemans BME, Swieringa F, Solari FA, Peng B, Thomassen SCM, Clemetson KJ, Farndale RW, Dubois LJ, Sickmann A, Zahedi RP, Ahrends R, Biessen EAL, Heemskerk JWM, Sluimer JC and Kuijpers MJE. Plasma lipid environment in hyperlipidemic and hypercholesterolemic mice enhances platelet and coagulation activation independent of age. $4^{\text {th }}$ European Platelet Network Conference (EUPLAN), Bruges, Belgium, 2018.

6. Tullemans BME, Veninga A, Fernández DI, Aarts MJB, van der Meijden PEJ, Heemskerk JWM and Kuijpers MJE. Multiparameter screening for platelet-inhibitory effects of tyrosine kinase inhibitors used for cancer treatment. The Platelet Society Meeting, Cambridge, United Kingdom, 2019.

7. Veninga A, Baaten CCFMJ, Tullemans BME, Kuijpers MJE, Heemskerk and van der Meijden PEJ. The effects of platelet agonists and priming on platelet populations. The Platelet Society Meeting, Cambridge, United Kingdom, 2019. 
8. Tullemans BME, Nagy M, Sabrkhany S, Griffioen AW, oude Egbrink MGA, Aarts MJ, Heemskerk JWM and Kuijpers MJE. Anti-platelet effects of tyrosine kinase inhibitors used for cancer treatment: pazopanib as a proof of principle. Dutch Association for Thrombosis and Haemostasis (NVTH) Symposium, Koudekerke, The Netherlands, 2019.

9. Tullemans BME, Fernández DI, Veninga A, Aarts MJB, van der Meijden PEJ, Heemskerk JWEM and Kuijpers MJE. Tyrosine kinase inhibitor sunitinib delays platelet-induced coagulation: additive effects of aspirin. Congress of the International Society on Thrombosis and Haemostasis (ISTH), Milan, Italy, 2020.

10. Brouns SLN, Tullemans BME, Perrella G, Campello E, Spiezia L, van Geffen JP, Kuijpers MJE, van Oerle R, Spronk HMH, Castoldi E, van der Meijden PEJ, Simioni P and Heemskerk JWM. Paradoxical platelet inhibitory effect of protein $\mathrm{C}$ deficiency in shear-dependent thrombus and fibrin formation. Congress of the International Society on Thrombosis and Haemostasis (ISTH), Milan, Italy, 2020.

11. Fernández DI, Provenzale I, Tullemans BME, van Groningen J, Heemskerk JWM, van den Hurk $\mathrm{H}$ and Kuijpers MJE. Disclosing inhibitory profiles of small molecule compounds through ITAM-linked or G-protein couple receptors using high-throughput calcium flux measurement. Congress of the International Society on Thrombosis and Haemostasis (ISTH), Milan, Italy, 2020.

12. Veninga A, Baaten CCFMJ, Tullemans BME, Kuijpers MJE, Heemskerk JWM and van der Meijden PEJ. The effects of platelet agonists and priming on platelet populations. Congress of the International Society on Thrombosis and Haemostasis (ISTH), Milan, Italy, 2020.

13. Handtke S, Tullemans BME, Brouns SLN, Veninga A, Aurich K, Greinacher A, Heemskerk JWM and Thiele T. Large and small platelets differently contribute to whole-blood thrombus formation. Congress of International Society on Thrombosis and Haemostasis (ISTH), Milan, Italy, 2020.

14. Veninga A, Baaten CCFMJ, Tullemans BME, De Simone I, Kuijpers MJE, Heemskerk JWM and van der Meijden PEJ. Analysis of platelet populations following platelet activation and priming using dimensionality reduction and automated clustering tools. The $2^{\text {nd }}$ Platelet Society (Virtual) Meeting, Keele, United Kingdom, 2021.

15. Tullemans BME, Brouns SLN, Swieringa F, Sabrkhany S, van den Berkmortel FWPJ, Peters NAJB, de Bruijn P, Koolen SLW, Heemskerk JWM, Aarts MJB and Kuijpers MJE. Quantitative and qualitative changes in platelet traits of sunitinib-treated patients with renal cell carcinoma in relation to circulating sunitinib levels: a proof-of-concept study. Congress of the international Society on Thrombosis and Haemostasis (ISTH), Philadelphia, USA, 2021.

16. Hrdinova J, Fernández DI, Ercig B, Tullemans BME, Suylen DPL, Agten SM, Jurk K, Hackeng TM, Vanhoorelbeke K, Voorberg J, Reutelingsperger CPM, Wichapong K, Heemskerk, JWM and Nicolaes GAF. Structure-based design of cyclic glycoprotein Iba-derived peptides 
affecting platelet interaction with von Willebrand factor under shear conditions. Congress of the international Society on Thrombosis and Haemostasis (ISTH), Philadelphia, USA, 2021.

17. Veninga A, Handtke S, Tullemans BME, Brouns SLN, Greinacher A, Heemskerk JWM, van der Meijden PEJ and Thiele T. Highly reactive juvenile platelets express higher levels of GPVI in a size related manner. Congress of the international Society on Thrombosis and Haemostasis (ISTH), Philadelphia, USA, 2021.

\section{Oral and poster presentations}

1. Tullemans BME. Platelet procoagulant activity is inhibited by the tyrosine kinase inhibitor pazopanib in vitro and in renal cell carcinoma patients. Centre for Thrombosis and Haemostasis (CTH) retreat, Mainz, Germany, 2017 (oral).

2. Tullemans BME. Platelet procoagulant activity is inhibited by tyrosine kinase inhibitor pazopanib in vitro and in renal cell carcinoma patients. $1^{\text {st }}$ Italian-UK platelet meeting, Bath, United Kingdom, 2017 (poster).

3. Tullemans BME. Tyrosine kinase inhibitor pazopanib inhibits platelets procoagulant activity in vitro and in cancer patients. Congress of the International Society on Thrombosis and Haemostasis (ISTH), Berlin, Germany, 2017 (poster).

4. Tullemans BME. Anti-platelet effects of tyrosine kinase inhibitors used for cancer treatment: pazopanib as a proof of principle. 4th European Platelet Network Conference (EUPLAN), Bruges, Belgium, 2018 (poster).

5. Tullemans BME. Multiparameter screening for platelet-inhibitory effects of tyrosine kinase inhibitors used for cancer treatment. The Platelet Society Meeting, Cambridge, United Kingdom, 2019 (poster).

6. Tullemans BME. Tyrosine kinase inhibitor sunitinib delays platelet-induced coagulation: additive effects of aspirin. Congress of the International Society on Thrombosis and Haemostasis (ISTH), Milan, Italy, 2020 (poster).

7. Tullemans BME. Quantitative and qualitative changes in platelet traits of sunitinib-treated patients with renal cell carcinoma in relation to circulating sunitinib levels: a proof-ofconcept study. Congress of the international Society on Thrombosis and Haemostasis (ISTH), Philadelphia, USA, 2021 (poster). 

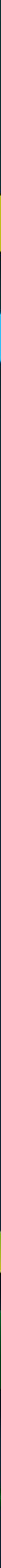
Acknowledgements 
"It always seems impossible until it's done" van Nelson Mandela, voor mij een toepasselijke uitspraak. Nooit had ik gedacht een PhD te gaan doen, laat staan ook te voltooien. Gelukkig heb ik deze kans gekregen en ben ik dit avontuur aangegaan, maar dit proefschrift zou nooit tot stand zijn gekomen zonder de hulp en steun van veel mensen die ik daar graag voor zou willen bedanken.

Om te beginnen wil ik mijn promotieteam graag bedanken voor de mooie kans die zij mij gegeven hebben en voor al hun hulp in deze periode.

Mijn promotor Prof. Johan Heemskerk, beste Johan. Bij jou stond altijd de deur open en kon iedereen altijd binnenlopen voor vanalles en nog wat. Soms maakte dit het wel lastig, want daardoor was je vaak druk in gesprek en soms moeilijk te vangen voor een brainstorm. Toch was je altijd bereid om mee te denken over alle en dus ook mijn projecten om weer nieuwe experimenten of insteken aan te dragen om er een goed verhaal van te maken. Het blijft indrukwekkend dat je zo makkelijk tussen alle projecten wist te wisselen en iedereen bleef helpen. Dankjewel voor al je tijd, ideeën en hulp tijdens mijn PhD.

Mijn co-promotor en dagelijks begeleider Dr. Marijke Kuijpers, beste Marijke. Zonder jouw aanbod was ik (mogelijk) nooit aan mijn PhD begonnen en had ik het zeker niet af kunnen krijgen. Ik ben je nog steeds heel dankbaar dat je mij bijna 6 jaar geleden hebt aangenomen als stagiair en mij daarna gelukkig nog niet kwijt wilde waardoor ik deze mooie uitdaging aan ben kunnen gaan! Je hebt me geholpen en gesteund, we hebben veel kunnen lachen, samen proeven kunnen doen en een hoop werk verzet. Ook bij jou stond de deur altijd open, waar ik ook vaak gebruik van heb gemaakt voor zowel de ups en downs van experimenten maar ook om gezellig te kletsen. Nu tijdens het afronden kon ik ook niet zonder je, hier heb je een grote rol voor mij in gespeeld en je hebt me door deze laatste tijd heen geholpen. Dankjewel voor alles wat je voor mij hebt gedaan en mij geleerd hebt!

Mijn co-promotor Dr. Maureen Aarts, beste Maureen. Als medisch oncoloog heb je ons geholpen met het includeren van patiënten in verschillende studies, maar tevens ook gezorgd voor een klinisch oogpunt en het enthousiast meedenken tijdens verschillende vergaderingen. Hartelijk dank voor alle hulp en patiënten inclusies die hebben gezorgd voor het tot stand komen van mijn proefschrift.

Dear members of the assessment committee, Prof. Hugo ten Cate, Prof. Gerard Bos, Prof. Judith Sluimer, Dr. Sonia Severin and Dr. Astrid van der Veldt, thank you very much for reviewing my thesis.

Een groot dankjewel voor de artsen vanuit Weert (Dr. Natascha Peters en Ted Goossens), Sittard (Dr. Franchette van de Berkmortel en Paul Schormans) en Eindhoven (Dr. Dorith Claushuis en Dr. Martin Nijziel) voor het meehelpen includeren van patiënten. But also a big 
appreciation to all the collaborators of this thesis I was able to work with in Maastricht (Prof. Miriam oude Egbrink), Amsterdam (Prof. Arjan Griffioen, Dr. Alex de Vos, Valentine Léopold and Marieke ten Brink), Rotterdam (Dr. Stijn Koolen and Peter de Bruijn), Aachen (Dr. Emiel van der Vorst, Dr. Sanne Maas and Linsey Peters), Münster (Prof. Johannes Eble) and Padua (Prof. Paolo Simioni, Dr. Luca Spiezia and Dr. Elena Campello). But also appreciation to the other collaborators in Maastricht, Greifswald, Mainz, Liège, Cambridge, Birmingham and many more for the interesting projects and publications. Thank you all very much for the opportunity, help and results. En natuurlijk mag ik alle vrijwilligers niet vergeten die gedurende de jaren bloed hebben gedoneerd of bloed hebben geprikt voor het mogelijk maken van zo goed als al mijn experimenten. Ook zonder hen was dit proefschrift nooit tot stand gekomen.

Mijn paranimfen, Louet en Mieke, ook jullie zijn belangrijk geweest in deze periode en ik zou jullie hier graag voor willen bedanken. Lieve Louet, ik ken jou al vanaf de middelbare school, maar pas tijdens onze opleiding bij de Fontys en bij de universiteit zijn we echt bevriend geraakt. We hebben samen de liefde voor de EFF gevonden en deze uitjes, maar ook de workshops en gezellige momentjes samen hebben me zeker wel helpen ontspannen. Ik kijk uit naar nog meer leuke momenten samen, maar bovenal naar de bruiloft van jou en Nick! Lieve Mieke, ook jij hebt op dezelfde middelbare school gezeten, maar bent een hele andere richting op gegaan. Dat ik jou als collega en kamergenootje mocht verwelkomen was een hele leuke verassing! Je hebt me veel geholpen zowel persoonlijk, als in het lab. Je bent een hele grote steun voor mij geweest. Ik ben heel blij dat we bevriend zijn geraakt na al die jaren. Ook voor jou is het eind van je PhD in zicht, dus nog heel veel succes met de laaste loodjes en ik hoop ook daarna nog gezellig af te kunnen spreken!

Natalie, not only a colleague and a friend, but also my "bonus paranymph"! I am really happy you turned out to be one of my roomies. From the day you arrived we had a lot of (fun) talks, great laughs, nice dinners and so much more. Thank you for your amazing personality, for being there and for judging things whenever needed. You really were a big support and helped me a lot. Good luck with your PhD, you know where to find me if you need help! Let's make more memories together in the future!

Mijn andere (oud-)kamergenootjes, Sanne \& Ankie, wil ik ook graag bedanken. De tijd dat ik met jullie samen heb mogen doorbrengen, de gesprekken, de lekkere dinertjes bij Novo, maar ook al jullie hulp is zeer gewaardeerd. Ik heb me er altijd thuis gevoeld! Natuurlijk zijn er meer mensen binnen het "superplatelet team" die ik niet mag vergeten. Alicia, dankjewel voor de leuke uitjes buiten werk of de appjes wanneer je een serie had gevonden die ik perse moest kijken. Maar ook je hulp en de samenwerking tijdens mijn ambitieuze experimenten. Het waren altijd gezellige dagen en hebben heel wat gelachen en gepraat! Delia, also you helped me a lot in 
the lab when I was planning to do massive experiments or when I was facing Flexstation-related problems. A big thank you for helping and keeping up with me, but also for the fun times and talks! Daniëlle, ook jij bedankt voor de gezellige tijden en gesprekken, de koffie momentjes, je onvergetelijke acties en je hulp tijdens het afronden. Frauke, we konden elkaar soms achter het behang plakken, maar op het eind van de dag wisten we wat we aan elkaar hadden. Dankjewel voor de leuke en gezellige dagen op het werk en we weten elkaar te vinden! Titus, bedankt voor alle groepsuitjes die je georganiseerd hebt om de boel weer een beetje op te leuken! Maar ook voor de leuke gesprekken en jouw kennis van technologie (eindelijk iemand die het snapt!). Magdi and Constance, although you both are already finished for a while, luckily, you are still part of the biochemistry department. You both were a great inspiration when I started at the department. I'm really grateful for all the things you taught me, but also that I could still come to your offices and ask for help when needed until the end. En Constance, natuurlijk ook dankjewel voor de hulp tijdens de revisies! Judith en Paola, dankjewel voor jullie kritische kijk op de papers tijdens onze samenwerkingen en jullie vertrouwen in mij voor die korte tijd als manusje van alles. Simone, dankjewel voor al de gezelligheid en je hulp in het lab. Ondanks dat jouw ongelukje ervoor zorgde dat ik langer mocht blijven, ben ik blij dat je nu weer naar de universiteit kan komen en we nog wat kleine dingen samen hebben kunnen verbeteren in het lab. Also thanks for everything to the other (ex-)colleagues of the superplatelet team: Tom, Siamack, Ilaria, Isabella, Jingnan, Claudia,

\section{Gina, Giorgia, Jinmi, Stefano, Chukiat and Hilaire.}

Alexandra. Annemiek en Danique, hoewel jullie geen onderdeel waren van het superplatelet team, waren jullie wel veel in de weer met bloedplaatjes. Alexandra, jij stond altijd klaar om te helpen, te brainstormen, of om te sparren over aspirine-donoren. We hebben over veel dingen dezelfde gedachten en hebben veel goede gesprekken gehad. Dankjewel hiervoor, maar natuurlijk ook voor de koffiemomentjes, de gezelligheid en het geruststellen. Annemiek, met jou heb ik het geluk gehad een complete studie te mogen doen. Het waren leuke en gezellige dagen vol experimenten, rare uitkomsten en breinbrekers voordat we klaar waren. Wat bewonder ik jou enorm hoe jij overal mee om gaat en doorzet! Heel erg bedankt voor alle gezelligheid en alle koffietjes. Heel veel succes met jouw boekje, maar dat gaat zeker lukken! Danique, wat blijkt een wereld toch klein te zijn, ook jij komt uit Weert en ik kende je van gezicht toen je bij de afdeling biochemie begon! In deze tijd hebben we het gezellig gehad op werk, in de trein en tijdens de koffiemomentjes. Daarnaast hebben we veel ergenissen kunnen delen over de aggregometer. Dankjewel!

Another thanks to all the people from the biochemistry department I worked with during the years for all the fun times, the borrels and annual "Day-Out" events!

Niet alleen collega's zijn belangrijk voor support en steun tijdens deze jaren en het maken van mijn proefschrift. Ook de afleiding of de hulp van vrienden en familie is van groot belang. Lieve 
Lisa, wat ben ik blij met onze vriendschap sinds de basisschool. We spreken niet zo vaak af als we zouden willen, maar ik geniet iedere keer weer van de etentjes of de momentjes samen. Wat ben ik trouwens blij dat je mij hebt geholpen met het uitvogelen van InDesign (ook al kon het alleen even digitaal) en me op weg te helpen met mijn proefschrift. Heel erg bedankt hiervoor (het heeft veel ergenissen gescheeld)! Hopelijk kunnen we snel weer wat leuks doen en naar jullie huisje komen kijken als het af is! Britt, Mike, Nick, Xander en Louet, tijdens onze jaren bij de Fontys zijn we altijd een hecht clubje geweest. Ook nu proberen we vaak wat te plannen, maar wat blijft een datum vinden toch soms moeilijk. Toch wil ik ook jullie bedanken voor de keren dat we gezellig wat hebben kunnen zitten drinken of Lego Movie hebben zitten kijken. Tijd voor een nieuwe datum en meer van deze momenten. Guus, Janou, Dennis, Maurice, Niels en Kelly, al jaore brînge vae beejeîn doeër, vere vae uutgebreijdj vastelaovendj en zeen de stapaovundje haole klot. Saame gaon vae op weekendjes ewèg en bezeuke vae brouweri-jje of (luxe) restaurants. Det hieët vör mich altiêd gezörgdj vör de noeëdige knoûwel en det ich met hieël waat ângers gaondje kos zeen dan wêrk. Dânke vör 't gelach, gezwets en d'n hieële tispel! Op nao 't volgendje fieëstje!

Je kan ze misschien niet kiezen, maar voor mij gaat er niets boven familie. Pap, mam en Annette, mer ounog Joost en Lenn, mien alike femiêlie en de "kaoje kânt" (ouch waal bekindj as schoeënfemiêlie). Dânke vör al uch belangstelling in waat ich in al di-j jaore heb gedaon, ouch al waas en es det dék neet gemaekelik te begriêpe. Dânke vör de loêsterendje oeëre en 't gewoeën beejeîn zeen met femiêlie, wi-j koffie beej opa, ' $t$ bbq'en of lekker online pubquizzen. Pap, mam en Annette dânke vör al uch vertrowwe en stuuën, det ich aldaag en vör eeder wissewasje kos belle of advies kos vraoge, vör as ich input noeëdig haaj of gewoeën effekes ewég woej. Op eîne zîn, dânke vör de hieële santekraom.

En dan as léste mer zieëker neet de minste, Jasper. Ich weît hieël zieëker det ich dit neet zónger uch haaj gekosj. Waat dejjae ammaol gedaon hetj is neet eins ammaol op te neume. Boete dejjae al 't hoêshaoje hetj gedaon en mich oeëveral beej hetj geholpe waat kos, hejje oug vör Hop en Lola gezörgdj en alles drejjendj weite te haoje. Ich haaj geîn baeter emes naeve mich kinne hebbe staon as uch en ich bin uch dan ouch dânkbaar vör alles. Noow weer same geneete vuuer vae 't kaomendj aventuër weer aangaon.

\section{Thank you!}

